\title{
Homogeneous hydrogenation with a cobalt/tetraphosphine catalyst: a superior hydride donor for polar double bonds and $N$-heteroarenes
}

Ya-Nan Duan ${ }^{\dagger}$, , Xiaoyong Du ${ }^{\dagger}, \|$, Zhikai Cui ${ }^{\dagger}$, Yiqun Zeng ${ }^{\dagger}$, Yufeng Liu ${ }^{\dagger}$, Tilong Yang ${ }^{\dagger}$, Jialin Wen ${ }^{*} \dagger, \dagger$ and Xumu Zhang ${ }^{*} \dagger$

${ }^{\dagger}$ Shenzhen Grubbs Institute and Department of Chemistry, Southern University of Science and Technology, Shenzhen, China;

* Academy for Advanced Interdisciplinary Studies, Southern University of Science and Technology, Shenzhen, China; "These authors contributed equally.

\section{Contents}

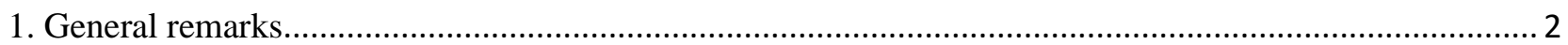

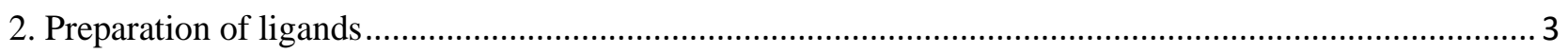

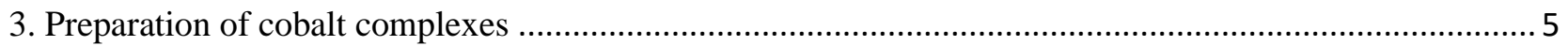

4. General procedure for cobalt catalyzed hydrogenation .................................................................. 7

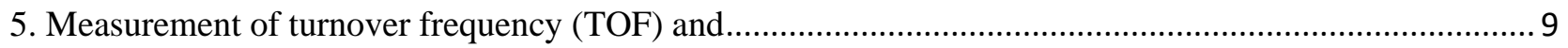

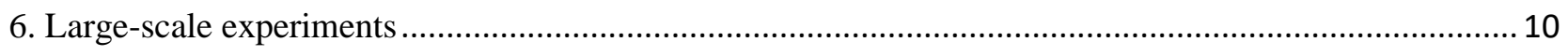

7. Moisture sensitivity and TEMPO-inhibited experiments ..................................................................... 11

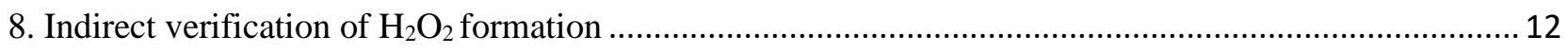

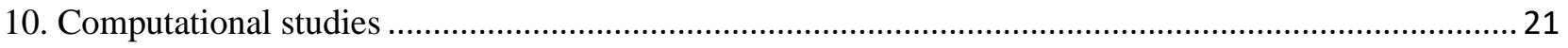

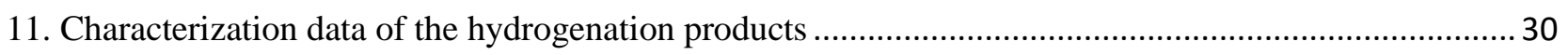

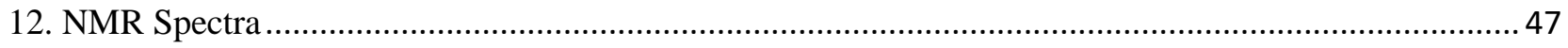

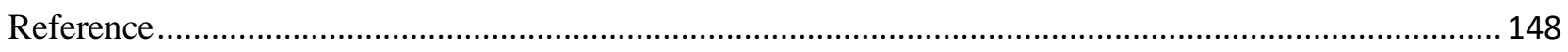




\section{General remarks}

All the reactions dealing with air- or moisture- sensitive compounds were carried out in a dry reaction vessel under an argon atmosphere or in an argon-filled glove box. Unless otherwise noted, all reagents and solvents were purchased from commercial suppliers without further purification. Ethyl ether was dried with sodium chips and indicated by benzophenone. Other anhydrous solvents were purchased from J\&K Chemical and degassed by bubbling argon over a period of $30 \mathrm{~min}$. Purification of products was carried out by flash chromatography using silica gel (200-300 mesh). Deuterated solvents were purchased from Cambridge Isotope Laboratories. There solvents were degassed by three freeze-pump-thaw cycles. ${ }^{1} \mathrm{H}$, ${ }^{13} \mathrm{C}$ and ${ }^{31} \mathrm{P}$ NMR spectra were obtained at room temperature on a Bruker AV400 $\mathrm{MHz}$ or a Bruker AV600 $\mathrm{MHz}$ spectrometer with chemical shifts (d) referenced to the residual solvent signal. Chemical shifts were reported upfield to TMS (0.00 ppm) for ${ }^{1} \mathrm{H}$ NMR and relative to $\mathrm{CDCl}_{3}(77.0 \mathrm{ppm})$ for ${ }^{13} \mathrm{C}$ NMR. Purification of products was carried out by flash chromatography using silica gel (200-300 mesh). Thin layer chromatography was carried out using silica gel plates from Merck (GF254). $\mathrm{Co}\left(\mathrm{BF}_{4}\right)_{2} \cdot 6 \mathrm{H}_{2} \mathrm{O}$ was purchased from Aldrich. Other cobalt precursors in this work were all purchased from Alfa Aesar. 


\section{Preparation of ligands}

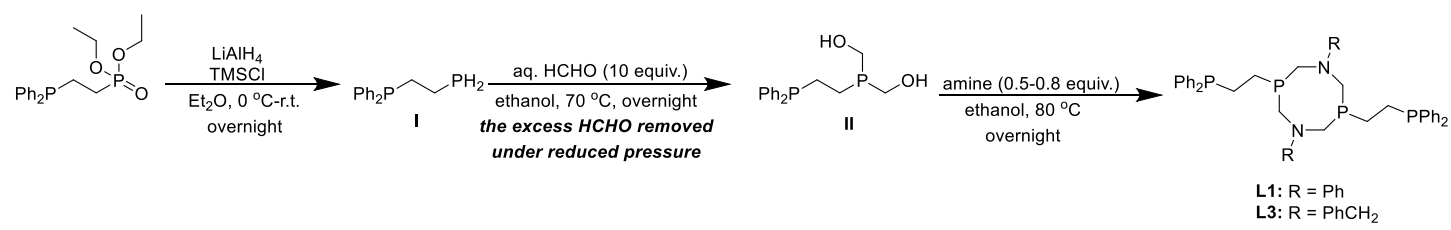

\subsection{Modified protocol for synthesis of diphenyl(2-phosphinoethyl)phosphine (I)}

In a modification of the previously reported procedure ${ }^{[1]}$, solid $\mathrm{LiAlH}_{4}(3.2 \mathrm{~g}, 0.084 \mathrm{~mol}, 2.9$ equiv.) was added in portions to a solution of trimethylsilylchloride $\left(10.0 \mathrm{~mL}, 0.079 \mathrm{~mol}, 2.7\right.$ equiv) in $\mathrm{Et}_{2} \mathrm{O}(150 \mathrm{~mL})$ at $0{ }^{\circ} \mathrm{C}$ in a $250 \mathrm{~mL}$ Schlenk flask. The mixture was stirred at ambient temperature for $1.5 \mathrm{~h}$, then the flask was cooled to $0{ }^{\circ} \mathrm{C}$ again and a solution of diethyl(2-(diphenylphosphino)ethyl)phosphonate $(10.2 \mathrm{~g}, 0.029 \mathrm{~mol})$ in $\mathrm{Et}_{2} \mathrm{O}(50 \mathrm{~mL})$ was added slowly via syringe to the stirring reaction mixture, which was then allowed to warm to ambient temperature. After stirring for $24 \mathrm{~h}$, the flask was cooled in an ice bath, then the reaction was quenched sequentially with degassed methanol. The resulting mixture was evaporated to dryness. The residue was rinsed with degassed hexane $(100 \mathrm{~mL})$ and filtered via diatomite plug and the salt was washed with degassed hexane $(20 \mathrm{~mL} \times 3)$. Then the filtrate was combined and evaporated under reduced pressure to afford the title phosphine, which was used directly in the next step.

\subsection{Synthesis of bis(hydroxymethyl)(2-diphenylphosphinoethyl)-phosphine (II)}

Diphenyl(2-phosphinoethyl)phosphine (3.04 g, $0.0123 \mathrm{~mol})$, degassed formaldehyde (42\% aqueous solution, 9.8 equiv), and EtOH $(100 \mathrm{~mL})$ were combined in a $250 \mathrm{~mL}$ Schlenk flask. The mixture was heated overnight in a $65{ }^{\circ} \mathrm{C}$ oil bath, then the mixture was evaporated and concentrated to dryness. The resulting oil was evacuated while heating at $60{ }^{\circ} \mathrm{C}$ overnight, which afforded bis(hydroxymethyl)(2-phosphinoethyl)phosphine $(2.20 \mathrm{~g})$ as a vicious liquid. The ${ }^{1} \mathrm{H}$ and ${ }^{31} \mathrm{P}\left\{{ }^{1} \mathrm{H}\right\}$ NMR spectra matched the literature values ${ }^{[1]}$.

\subsection{Synthesis of tetradentate phosphine ligand $\mathrm{P}^{2 \mathrm{C}-\mathrm{PPh}_{2}}{ }_{2} \mathrm{~N}^{\mathrm{Ph}}$ (L1)}

Crude bis(hydroxymethyl)(2-phosphinoethyl)phosphine (2.99 g) was dissolved in EtOH $(60 \mathrm{~mL})$ in a $100 \mathrm{~mL}$ Schlenk flask. Aniline $(0.88 \mathrm{~mL}, 9.7 \mathrm{mmol})$ was added to the solution via syringe, then the flask was heated overnight in a $65{ }^{\circ} \mathrm{C}$ oil bath, causing a white precipitate to form. After cooling to room temperature, the mixture was filtered, and the solid was rinsed with EtOH $(20 \mathrm{~mL})$ and $\mathrm{Et}_{2} \mathrm{O}(2 \times 20 \mathrm{~mL})$ to afford $\mathrm{P}^{2 \mathrm{C}-\mathrm{PPh} 2}{ }_{2} \mathrm{~N}^{\mathrm{Ph}}{ }_{2}(\mathbf{L 1})(1.69 \mathrm{~g}, 2.33 \mathrm{mmol}, 48 \%$ yield based on aniline) as a white powder. ${ }^{1} \mathrm{H}$ NMR $\left(400 \mathrm{MHz}, \mathrm{CDCl}_{3}\right) \delta 7.44(\mathrm{ddt}, J=7.2,5.4,2.3 \mathrm{~Hz}, 4 \mathrm{H}), 7.41-7.32(\mathrm{~m}, 6 \mathrm{H}), 7.19$ (dd, $J=8.8,7.2 \mathrm{~Hz}, 2 \mathrm{H}), 6.67$ (d, $J=8.6 \mathrm{~Hz}, 3 \mathrm{H}), 4.14$ (t, $J=14.2 \mathrm{~Hz}, 2 \mathrm{H}), 3.36$ (dd, $J=15.0,4.4 \mathrm{~Hz}, 2 \mathrm{H}), 2.34-2.21$ $(\mathrm{m}, 2 \mathrm{H}), 1.44(\mathrm{~d}, J=8.4 \mathrm{~Hz}, 2 \mathrm{H}) ;{ }^{31} \mathrm{P}$ NMR $\left(162 \mathrm{MHz}, \mathrm{CDCl}_{3}\right) \delta-12.5(\mathrm{~d}, J=25.9 \mathrm{~Hz}),-46.4(\mathrm{~d}, J=25.9 \mathrm{~Hz})$. The ${ }^{1} \mathrm{H}$ and ${ }^{31} \mathrm{P}\left\{{ }^{1} \mathrm{H}\right\}$ NMR spectra matched the literature values ${ }^{[1]}$.

\subsection{Synthesis of tetradentate phosphine ligand $\mathrm{P}^{3 \mathrm{C}-\mathrm{PPh}_{2}} \mathrm{~N}^{\mathrm{Ph}}{ }_{2}$ (L2)}

This compound was prepared in a manner analogous to that described for $\mathbf{L 1}$ and was isolated as white powder in $40 \%$ yield based on aniline. ${ }^{1} \mathrm{H}$ NMR (400 MHz, Chloroform- $d$ ) $\delta 7.44(\mathrm{t}, J=6.9 \mathrm{~Hz}, 8 \mathrm{H}), 7.34(\mathrm{~d}, J=6.2 \mathrm{~Hz}, 12 \mathrm{H}), 7.14(\mathrm{t}, J$ $=7.8 \mathrm{~Hz}, 4 \mathrm{H}), 6.65(\mathrm{~s}, 2 \mathrm{H}), 6.58(\mathrm{~d}, J=8.1 \mathrm{~Hz}, 4 \mathrm{H}), 4.09(\mathrm{t}, J=14.1 \mathrm{~Hz}, 4 \mathrm{H}), 3.39(\mathrm{dd}, J=15.0,4.4 \mathrm{~Hz}, 4 \mathrm{H}), 2.22(\mathrm{t}, J$ 
$=7.7 \mathrm{~Hz}, 4 \mathrm{H}), 1.75(\mathrm{p}, J=7.8 \mathrm{~Hz}, 4 \mathrm{H}), 1.52(\mathrm{t}, J=7.9 \mathrm{~Hz}, 4 \mathrm{H}) ;{ }^{31} \mathrm{P}$ NMR $(162 \mathrm{MHz}$, Chloroform- $d$ ) $\delta$-17.28, -51.71;

${ }^{13}$ C NMR (101 MHz, Chloroform- $d$ ) $\delta$ 145.9, 138.5, 138.3, 132.9, 132.7, 129.2, 128.8, 128.6, 128.5, 116.6, 112.4, 56.8, 56.6, 29.8, 22.8. The ${ }^{1} \mathrm{H}$ and ${ }^{31} \mathrm{P}\{1 \mathrm{H}\}$ NMR spectra matched the literature values ${ }^{[1]}$.

\subsection{Synthesis of tetradentate phosphine ligand $\mathrm{P}^{2 \mathrm{C}-\mathrm{PPh}_{2}} \mathrm{~N}^{\mathrm{Bn}}{ }_{2}(\mathrm{~L} 3)$}

The synthetic procedure was the same as that of $\mathbf{L 1}$ except that benzyl amine was used instead of aniline. Ligand $\mathrm{P}^{2 \mathrm{C}-\mathrm{PPh} 2}{ }_{2} \mathrm{~N}^{\mathrm{Bn}}{ }_{2}$ (L3) was afforded as a white powder (1.80 g, $2.34 \mathrm{mmol}, 49 \%$ based on benzyl amine). ${ }^{1} \mathrm{H}$ NMR (400 MHz, Chloroform- $d$ ) $\delta 7.33-7.22(\mathrm{~m}, 23 \mathrm{H}), 7.18(\mathrm{q}, J=6.4 \mathrm{~Hz}, 7 \mathrm{H}), 3.99(\mathrm{~s}, 4 \mathrm{H}), 3.04(\mathrm{~d}, J=15.3 \mathrm{~Hz}, 8 \mathrm{H}), 1.84(\mathrm{~m}, 4 \mathrm{H})$, 1.06-0.98 (m, 4H); ${ }^{31} \mathrm{P}$ NMR (162 MHz, $\left.\mathrm{CDCl}_{3}\right) \delta-13.2$ (m), -69.3 (brs); ${ }^{13} \mathrm{C}$ NMR (101 MHz, $\left.\mathrm{CDCl}_{3}\right) \delta 138.1,137.9$, 132.8, 132.6, 129.3, 128.6, 128.4, 128.4, 127.3, 77.3, 77.0, 76.7, 60.3, 24.4, 22.2; HRMS: (ESI): $\mathrm{m} / \mathrm{z}$ calcd for $\mathrm{C}_{45} \mathrm{H}_{49} \mathrm{~N}_{2} \mathrm{P}_{4}[\mathrm{M}+\mathrm{H}]^{+}:$: 741.2841, found: 741.2830 . 


\section{Preparation of cobalt complexes}

\section{1 $\left[\mathrm{Co}^{\mathrm{II}}(\mathrm{L1})\left(\mathrm{CH}_{3} \mathrm{CN}\right)\right]\left(\mathrm{BF}_{4}\right)_{2}$}

Solid $\mathrm{Co}\left(\mathrm{BF}_{4}\right)_{2} \cdot 6 \mathrm{H}_{2} \mathrm{O}(1 \mathrm{mmol}, 340 \mathrm{mg})$ was dissolved in $5 \mathrm{~mL} \mathrm{CH}_{3} \mathrm{CN}$ to form a brown solution, which was stirred at room temperature for $20 \mathrm{~min}$. After that, the solution was added to a suspension of $\mathbf{L 1}(0.96 \mathrm{mmol}, 700 \mathrm{mg})$ in $\mathrm{CH}_{3} \mathrm{CN}$ $(50 \mathrm{~mL})$. The resulting dark brown solution was stirred for $3 \mathrm{~h}$, then the solvent was removed under vacuum. The resulting residue was stirred in hexane overnight and the mixture was filtered, and the brown solid was dried under vacuum to afford the title $\left[\mathrm{CO}^{\mathrm{II}}(\mathbf{L 1})\left(\mathrm{CH}_{3} \mathrm{CN}\right)\right]\left(\mathrm{BF}_{4}\right)_{2}(900 \mathrm{mg})$ in $94 \%$ yield. The ${ }^{1} \mathrm{H}$ NMR spectra data matched the literature values ${ }^{[1]}$.

\section{$3.2\left[\mathrm{Co}^{\mathrm{I}}(\mathrm{L1})\left(\mathrm{CH}_{3} \mathrm{CN}\right)\right]\left(\mathrm{BF}_{4}\right)$}

Deep brown solid $\left[\mathrm{Co}^{\mathrm{II}}(\mathbf{L} \mathbf{1})\left(\mathrm{CH}_{3} \mathrm{CN}\right)\right]\left(\mathrm{BF}_{4}\right)_{2}(100 \mathrm{mg}, 0.1 \mathrm{mmol})$ was added to a $100 \mathrm{~mL}$ shrek tube and dissolved in $20 \mathrm{~mL} \mathrm{CH}_{3} \mathrm{CN}$ to form a brown solution. $\mathrm{KC}_{8}(33 \mathrm{mg}, 0.24 \mathrm{mmol}$ ) was added in one portion to the above solution. The resulting suspension was stirred at room temperature for 24 hours. After that, the suspended solid was filtrated via diatomite plug and washed with dry $\mathrm{CH}_{3} \mathrm{CN}$ for three times. Then the filtrate was evaporated under reduced pressure and the residue was dissolved in dry THF and filtrated via diatomite plug and then removed the solvent under vacuum to afford the orange solid which was washed with $\mathrm{Et}_{2} \mathrm{O}$ for three times and the solid was collected and dried under vacuum to give $\left[\mathrm{Co}^{\mathrm{I}}(\mathrm{L} 1)\left(\mathrm{CH}_{3} \mathrm{CN}\right)\right]\left(\mathrm{BF}_{4}\right)$ in $50 \%$ yield $(47 \mathrm{mg})$. The ${ }^{1} \mathrm{H}$ NMR spectra data matched the literature values ${ }^{[1]}$.

\subsection{Trans-[(H) $\left.)_{2}-\mathrm{Co}^{\mathrm{III}}(\mathrm{L1})\right]\left(\mathrm{BF}_{4}\right)$}

In an argon glovebox, $\left[\mathrm{Co}^{\mathrm{I}}(\mathbf{L 1})\left(\mathrm{CH}_{3} \mathrm{CN}\right)\right]\left(\mathrm{BF}_{4}\right)(9.6 \mathrm{mg}, 0.01 \mathrm{mmol})$ was dissolved in THF-d 8 (500 $\left.\mu \mathrm{L}\right)$. Then the resulting dark red solution was transferred to a J. Young tube and sealed, and then the tube was immersed in liquid nitrogen and evacuated its head regions and refilled with dihydrogen via vacuum line. The above process was repeated for three times. Then, the tube was gradually warmed to room temperature and vortexed for several minutes. The J. Young tube was immersed in $60{ }^{\circ} \mathrm{C}$ oil bath for $12 \mathrm{~h}$. The dark red solution gradually became orange. Finally, ${ }^{1} \mathrm{H}$ and ${ }^{31} \mathrm{P}$ NMR were recorded. ${ }^{1} \mathrm{H}$ NMR $\left(400 \mathrm{MHz}, \mathrm{THF}-d^{8}\right) \delta 7.18(\mathrm{dd}, J=8.6,7.1 \mathrm{~Hz}, 4 \mathrm{H}), 7.11-6.89(\mathrm{~m}, 24 \mathrm{H}), 6.77(\mathrm{t}, J=7.3$ $\mathrm{Hz}, 2 \mathrm{H}), 4.11(\mathrm{~d}, J=14.0 \mathrm{~Hz}, 4 \mathrm{H}), 3.70(\mathrm{~d}, J=14.0 \mathrm{~Hz}, 4 \mathrm{H}), 2.52(\mathrm{~d}, J=23.2 \mathrm{~Hz}, 4 \mathrm{H}), 2.25(\mathrm{~d}, J=23.2 \mathrm{~Hz}, 4 \mathrm{H}),-11.48$ $(\mathrm{m}, 2 \mathrm{H}) ;{ }^{31} \mathrm{P}$ NMR $\left(162 \mathrm{MHz}, \mathrm{THF}-d_{8}\right) \delta 93.7(\mathrm{~d}, J=97.9 \mathrm{~Hz}), 90.5(\mathrm{~d}, J=98.0 \mathrm{~Hz}) .{ }^{13} \mathrm{C}$ NMR $\left(101 \mathrm{MHz}, \mathrm{THF}-d_{8}\right) \delta$ 133.5, 133.4, 133.4, 130.0, 129.8, 128.2, 128.1, 119.3, 117.0, 66.7, 66.5, 51.4, 51.2, 24.7, 24.5.

NMR spectroscopic data are consistent with assignment of the $\mathrm{H}_{2}$ addition product as trans- $\left[(\mathrm{H})_{2} \mathrm{Co}^{\mathrm{III}}(\mathbf{L} \mathbf{1})\right]\left(\mathrm{BF}_{4}\right)$ complex. A single upfield resonance is observed as a quintet at $-11.48 \mathrm{ppm}\left(2 \mathrm{H}, J_{P-H}=23.6 \mathrm{~Hz}\right)$ in the ${ }^{1} \mathrm{H} \mathrm{NMR}$ spectrum, corresponding to two magnetically equivalent hydride ligands having a similar scalar interaction with the inequivalent phosphorus groups.

\section{$3.4\left[\mathrm{Co}^{\mathrm{I}}(\mathrm{H})(\mathrm{L} 1)\right]$}

In an argon glovebox, $\left[\mathrm{Co}^{\mathrm{I}}(\mathbf{L 1})\left(\mathrm{CH}_{3} \mathrm{CN}\right)\right]\left(\mathrm{BF}_{4}\right)(9.6 \mathrm{mg}, 0.01 \mathrm{mmol})$ was dissolved in $\mathrm{CD}_{3} \mathrm{CN}(500 \mu \mathrm{L})$. Then the resulting orange solution was transferred to a J. Young tube and the tube was cooled at $-30{ }^{\circ} \mathrm{C}$ in the freezer for $30 \mathrm{~min}$. After that, $\mathrm{NaEt}_{3} \mathrm{BH}(1.0 \mathrm{M}$ in THF, $11 \mu \mathrm{L})$ was added via microsyringe to the above orange solution. The tube was gradually warmed to room temperature and vortexed for several minutes. Finally, the corresponding ${ }^{1} \mathrm{H}$ and ${ }^{31} \mathrm{P}$ NMR 
spectra were recorded. ${ }^{1} \mathrm{H}$ NMR (400 MHz, Acetonitrile- $\left.d_{3}\right) \delta 8.21-6.34(\mathrm{~m}, 30 \mathrm{H}), 3.93(\mathrm{~d}, J=11.9 \mathrm{~Hz}, 2 \mathrm{H}), 3.86(\mathrm{~s}$, 2H), 3.58 (s, 2H), 3.50 (s, 2H), 3.17 (d, $J=12.4 \mathrm{~Hz}, 2 \mathrm{H}), 3.05$ (d, $J=12.5 \mathrm{~Hz}, 2 \mathrm{H}), 2.59$ (s, 4H), -8.46 (br s, $J=15.0 \mathrm{~Hz}$, 1H); ${ }^{31}$ P NMR (162 MHz, Acetonitrile- $\left.d_{3}\right) \delta 84.02(\mathrm{~d}, J=60.4 \mathrm{~Hz}), 81.18(\mathrm{~d}, J=60.4 \mathrm{~Hz})$. 


\section{General procedure for cobalt catalyzed hydrogenation}

In an argon glovebox, a $5 \mathrm{~mL}$ vial equipped with a magnetic stirring bar, was added the required amount of catalyst solution (in $\mathrm{CH}_{3} \mathrm{CN}$ ), potassium hydroxide, substrates and the refereed solvent. The mixture was transferred to a stain-less autoclave and then was purged by three cycles of pressurization/venting with $\mathrm{H}_{2}(10 \mathrm{~atm})$ then pressurized with $\mathrm{H}_{2}(30 \mathrm{~atm})$ and disconnected from the $\mathrm{H}_{2}$ source. Then the autoclave was placed in an oil bath preheated to the desired temperature. After the desired reaction time, the autoclave was cooled in an ice bath, and the pressure was released slowly. The reaction mixture was diluted with DCM, filtered via a short plug and evaporated under reduced pressure. The resulting residue was analyzed via ${ }^{1} \mathrm{H}$ NMR using internal standard.

Condition optimization. A series of tetraphosphine $\left(\mathrm{P}^{\mathrm{nC}-\mathrm{PPh} 2}{ }_{2} \mathrm{~N}_{2} \mathrm{R}: \mathrm{n}=2\right.$ and $3 ; \mathrm{R}=\mathrm{Ph}$ and $\left.\mathrm{Bn}\right)$ ligands $(\mathbf{L 1 - 3})$ and $\mathrm{P}_{2} \mathrm{~N}^{\mathrm{Ph}}$ ligand (L4) were easily prepared in gram scales according to the reported procedure ${ }^{[1-2]}$. With these tetra- and bisphosphine ligands in hand, we commenced our study by testing the performance of various combinations of cobalt precursors and different phosphine ligands in the reaction of model substrate trans-cinnamaldehyde (1aa) under 30 bar of $\mathrm{H}_{2}$ at $60{ }^{\circ} \mathrm{C}$ in EtOH (Table S1). It is revealed that the length of carbon skeleton in the bisphosphines alters the bite angle when coordinated to metal and therefore influences the catalytic reaction. While $\mathrm{P}^{2 \mathrm{C}-\mathrm{PPh}}{ }_{2} \mathrm{~N}^{\mathrm{Ph}}{ }_{2}$ gave a full conversion, $\mathrm{P}^{3 \mathrm{C}-\mathrm{PPh} 2}{ }_{2} \mathrm{~N}^{\mathrm{Ph}}{ }_{2}$ which has longer side arms resulted in a diminished conversion (entry 2\&27). Switching the $N$-substitution group from phenyl to benzyl led to a similar reactivity, which suggested that the basicity of amine may do not influence this reaction (entry 24). Furthermore, employing $\mathrm{Co}\left(\mathrm{BF}_{4}\right)_{2} \cdot 6 \mathrm{H}_{2} \mathrm{O}$ with 2 equivalents of bisphosphine ligand $\mathbf{L} \mathbf{4}$ or dppe did not yield the desired allylic alcohol product 2aa efficiently (entries 25-26). Various cobalt precursors were evaluated, among which $\mathrm{Co}\left(\mathrm{BF}_{4}\right)_{2} \bullet 6 \mathrm{H}_{2} \mathrm{O}$ reacted as the most efficient one, although $\mathrm{CoCl}_{2}$ presented a similar activity ( $>95 \%$ conv. $90 \%$ yield). A control experiment in the absence of $\mathrm{KOH}$ revealed that the base is necessary in this chemical transformation (entry 1). The cobalt complex, $\left[\mathrm{Co}^{\mathrm{II}}(\mathrm{L1})\left(\mathrm{CH}_{3} \mathrm{CN}\right)\right]\left(\mathrm{BF}_{4}\right)_{2}(3)$, was readily synthesized by mixing an equimolar mixture of $\mathbf{L 1}$ and $\mathrm{Co}\left(\mathrm{BF}_{4}\right)_{2} \cdot 6 \mathrm{H}_{2} \mathrm{O}$. The reactions using 1 mol\% of $\mathbf{3}$ gave 2 aa in nearly quantitative yield after $24 \mathrm{~h}$.

\section{Table S1: Detailed conditions screening}

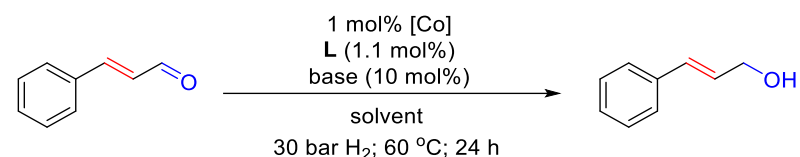

\begin{tabular}{|c|c|c|c|c|c|}
\hline Entry & precatalyst & base & solvent & Conv. $(\%)^{a}$ & Yield $(\%)^{a}$ \\
\hline 1 & $\mathrm{Co}\left(\mathrm{BF}_{4}\right)_{2} \cdot 6 \mathrm{H}_{2} \mathrm{O}$ & - & $\mathrm{EtOH}$ & 0 & 0 \\
\hline 2 & $\mathrm{Co}\left(\mathrm{BF}_{4}\right)_{2} \cdot 6 \mathrm{H}_{2} \mathrm{O}$ & $\mathrm{KOH}$ & $\mathrm{EtOH}$ & 100 & 99 \\
\hline 3 & $\mathrm{CoCl}_{2}$ & $\mathrm{KOH}$ & $\mathrm{EtOH}$ & 95 & 90 \\
\hline 4 & $\mathrm{CoBr}_{2}$ & $\mathrm{KOH}$ & $\mathrm{EtOH}$ & 90 & 90 \\
\hline 5 & $\mathrm{Co}(\mathrm{acac})_{2}$ & $\mathrm{KOH}$ & $\mathrm{EtOH}$ & 75 & 75 \\
\hline 6 & $\mathrm{Co}\left(\mathrm{ClO}_{4}\right)_{2} \cdot 6 \mathrm{H}_{2} \mathrm{O}$ & $\mathrm{KOH}$ & $\mathrm{EtOH}$ & 30 & 30 \\
\hline 7 & $\mathrm{Co}(\mathrm{OAc})_{2} \cdot 4 \mathrm{H}_{2} \mathrm{O}$ & $\mathrm{KOH}$ & $\mathrm{EtOH}$ & 0 & 0 \\
\hline 8 & $\mathrm{CoC}_{2} \mathrm{O}_{4}$ & $\mathrm{KOH}$ & $\mathrm{EtOH}$ & 0 & 0 \\
\hline 9 & $\mathrm{Co}\left(\mathrm{BF}_{4}\right)_{2} \cdot 6 \mathrm{H}_{2} \mathrm{O}$ & $\mathrm{KOH}$ & DCE & 0 & 0 \\
\hline
\end{tabular}




\begin{tabular}{|c|c|c|c|c|c|}
\hline 10 & $\mathrm{Co}\left(\mathrm{BF}_{4}\right)_{2} \cdot 6 \mathrm{H}_{2} \mathrm{O}$ & $\mathrm{KOH}$ & DCM & 0 & 0 \\
\hline 11 & $\mathrm{Co}\left(\mathrm{BF}_{4}\right)_{2} \cdot 6 \mathrm{H}_{2} \mathrm{O}$ & $\mathrm{KOH}$ & THF & 100 & 95 \\
\hline 12 & $\mathrm{Co}\left(\mathrm{BF}_{4}\right)_{2} \cdot 6 \mathrm{H}_{2} \mathrm{O}$ & $\mathrm{KOH}$ & $\mathrm{MeOH}$ & 95 & 95 \\
\hline 13 & $\mathrm{Co}\left(\mathrm{BF}_{4}\right)_{2} \cdot 6 \mathrm{H}_{2} \mathrm{O}$ & $\mathrm{KOH}$ & $i \mathrm{PrOH}$ & 100 & 99 \\
\hline 14 & $\mathrm{Co}\left(\mathrm{BF}_{4}\right)_{2} \cdot 6 \mathrm{H}_{2} \mathrm{O}$ & $\mathrm{KOH}$ & Toluene & 68 & 68 \\
\hline 15 & $\mathrm{Co}\left(\mathrm{BF}_{4}\right)_{2} \cdot 6 \mathrm{H}_{2} \mathrm{O}$ & $\mathrm{KOH}$ & $\mathrm{CH}_{3} \mathrm{CN}$ & 100 & 95 \\
\hline 16 & $\mathrm{Co}\left(\mathrm{BF}_{4}\right)_{2} \cdot 6 \mathrm{H}_{2} \mathrm{O}$ & $\mathrm{KOH}$ & 1,4-Dioxane & 100 & 99 \\
\hline 17 & $\mathrm{Co}\left(\mathrm{BF}_{4}\right)_{2} \cdot 6 \mathrm{H}_{2} \mathrm{O}$ & $\mathrm{LiOH}$ & $\mathrm{EtOH}$ & 100 & 90 \\
\hline 18 & $\mathrm{Co}\left(\mathrm{BF}_{4}\right)_{2} \cdot 6 \mathrm{H}_{2} \mathrm{O}$ & $\mathrm{NaOH}$ & $\mathrm{EtOH}$ & 100 & 92 \\
\hline 19 & $\mathrm{Co}\left(\mathrm{BF}_{4}\right)_{2} \cdot 6 \mathrm{H}_{2} \mathrm{O}$ & $\mathrm{CsOH}$ & $\mathrm{EtOH}$ & 100 & 94 \\
\hline 20 & $\mathrm{Co}\left(\mathrm{BF}_{4}\right)_{2} \cdot 6 \mathrm{H}_{2} \mathrm{O}$ & $\mathrm{K}_{2} \mathrm{CO}_{3}$ & $\mathrm{EtOH}$ & 100 & 98 \\
\hline 21 & $\mathrm{Co}\left(\mathrm{BF}_{4}\right)_{2} \cdot 6 \mathrm{H}_{2} \mathrm{O}$ & $t \mathrm{BuOK}$ & $\mathrm{EtOH}$ & 100 & 91 \\
\hline 22 & $\mathrm{Co}\left(\mathrm{BF}_{4}\right)_{2} \cdot 6 \mathrm{H}_{2} \mathrm{O}$ & DABCO & $\mathrm{EtOH}$ & 20 & 18 \\
\hline 23 & $\mathrm{Co}\left(\mathrm{BF}_{4}\right)_{2} \cdot 6 \mathrm{H}_{2} \mathrm{O}$ & $\mathrm{Et}_{3} \mathrm{~N}$ & $\mathrm{EtOH}$ & 0 & -- \\
\hline $24^{b}$ & $\mathrm{Co}\left(\mathrm{BF}_{4}\right)_{2} \cdot 6 \mathrm{H}_{2} \mathrm{O}$ & $\mathrm{KOH}$ & $\mathrm{EtOH}$ & 100 & 99 \\
\hline $25^{c}$ & $\mathrm{Co}\left(\mathrm{BF}_{4}\right)_{2} \cdot 6 \mathrm{H}_{2} \mathrm{O}$ & $\mathrm{KOH}$ & $\mathrm{EtOH}$ & 11 & n.d. \\
\hline $26^{d}$ & $\mathrm{Co}\left(\mathrm{BF}_{4}\right)_{2} \cdot 6 \mathrm{H}_{2} \mathrm{O}$ & $\mathrm{KOH}$ & $\mathrm{EtOH}$ & 92 & 25 \\
\hline $27^{e}$ & $\mathrm{Co}\left(\mathrm{BF}_{4}\right)_{2} \cdot 6 \mathrm{H}_{2} \mathrm{O}$ & $\mathrm{KOH}$ & $\mathrm{EtOH}$ & 60 & 56 \\
\hline
\end{tabular}

${ }^{a}$ The conversion and yields were determined via ${ }^{1} \mathrm{H}$ NMR using 1,2-dibromoethane as the internal standard. DABCO $=$ 1,4-diazabicyclo[2.2.2] octane; acac $=$ acetylacetonate; $\mathrm{DCE}=1,2$-dichloroethane; $\mathrm{DCM}=$ dichloromethane. ${ }^{b} 1.1 \mathrm{~mol} \%$ $\mathrm{P}^{2 \mathrm{C}-\mathrm{PPh}_{2}}{ }_{2} \mathrm{~N}^{\mathrm{Bn}}{ }_{2}$ was employed as the ligand. ${ }^{c} 2.2 \mathrm{~mol} \%$ 1,2-bis(diphenylphosphino)ethane (dppe) was employed as the ligand. ${ }^{d} .2 \mathrm{~mol} \% \mathrm{P}_{2} \mathrm{~N}^{\mathrm{Ph}}$ was employed as the ligand. ${ }^{e} 1.1 \mathrm{~mol} \% \mathrm{P}^{3 \mathrm{C}-\mathrm{PPh}_{2}}{ }_{2} \mathrm{~N}^{\mathrm{Ph}}{ }_{2}$ was employed as the ligand.
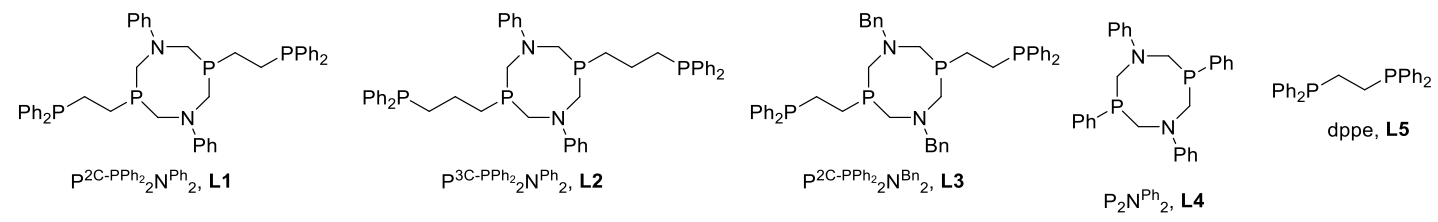


\section{Measurement of turnover frequency (TOF) and}

Hydrogenation reactions under pressurized condition were carried out on a Mettler-Toledo EasyMax 102 station with a 100-mL pressure vessel. The AFT-FTIR was performed with Mettler-Toledo ReactIR15 instrument. Experiment procedure: The vessel was dried by a heat gun and sealed afterwards. After 3 vacuum-argon-back-fill cycles, a mixture of 1bb $(20.0 \mathrm{mmol}), \mathbf{3}(0.02 \mathrm{mmol})$ and base $(0.20 \mathrm{mmol})$ in anhydrous ethanol was transferred into the vessel via a syringe. The vessel was pressurized with 30 atm hydrogen gas and stirred at $300 \mathrm{rpm}$ (magnetic stirring). The temperature was maintained at $60^{\circ} \mathrm{C}$ and the IR spectra were collected with such time interval as every 15 seconds. The reaction was monitored by tracing the consumption of $\mathbf{1 b b}$ at $1702 \mathrm{~cm}^{-1}$ (yellow line) and $1599 \mathrm{~cm}^{-1}$ (blue line). A baseline correction was applied before analysing the peak intensities. Reaction parameters were summarized as follow:

Table S2:

\begin{tabular}{|c|c|c|c|c|}
\hline temp & total vol. & conc. of $\mathbf{1 b b}$ & conc. of cat. & S/C \\
\hline $60^{\circ} \mathrm{C}$ & $23.0 \mathrm{~mL}$ & $0.87 \mathrm{M}$ & $8.7^{*} 10^{-4} \mathrm{M}$ & 1000 \\
\hline
\end{tabular}

When the reaction was performed at $60{ }^{\circ} \mathrm{C}$, this reaction was complete in five minutes, giving a TOF $\sim 12000 \mathrm{~h}^{-1}$. The crude data:

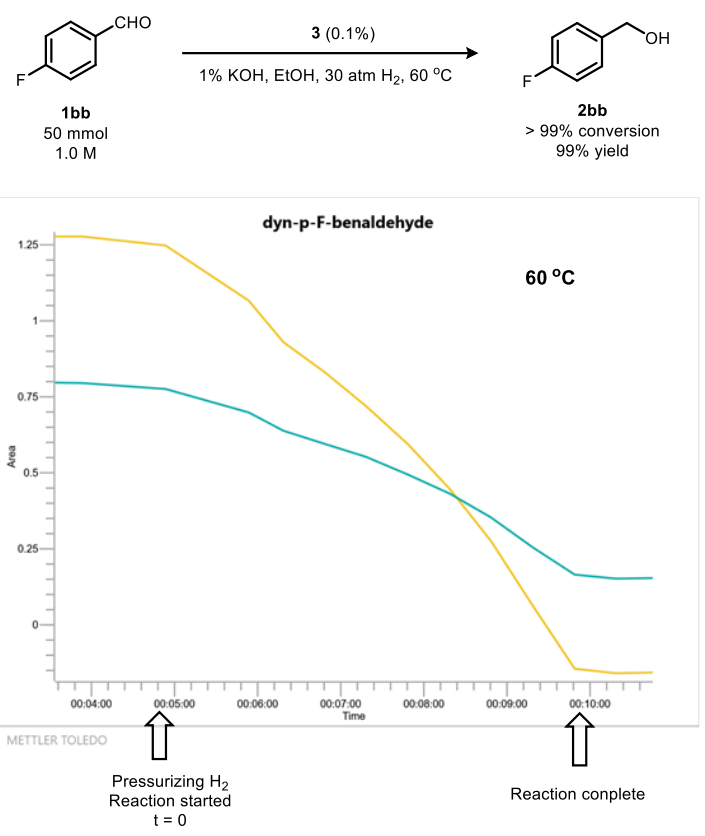

Figure S1. Measurement of turnover frequency for hydrogenation of 4-F-benzaldehyde using AFT-FTIR 


\section{Large-scale experiments}

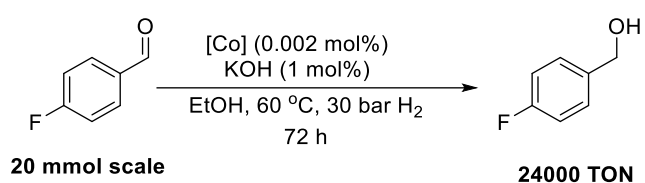

In an argon glovebox, a $50 \mathrm{~mL}$ vial equipped with a magnetic stirring bar, was added $40 \mu \mathrm{L}$ catalyst solution ([Co $\left.{ }^{\mathrm{II}}(\mathbf{L 1})\left(\mathrm{CH}_{3} \mathrm{CN}\right)\right]\left(\mathrm{BF}_{4}\right)_{2}: 0.01 \mathrm{M}$ in $\left.\mathrm{CH}_{3} \mathrm{CN}\right), p$-F-benzaldehyde $(20 \mathrm{mmol}, 2.48 \mathrm{~g}$ ), potassium hydroxide (1 mol\%, $11.2 \mathrm{mg}$ ) and $10 \mathrm{~mL}$ ethanol. Then the mixture was transferred to a stain-less autoclave and then was purged by three cycles of pressurization/venting with $\mathrm{H}_{2}(10 \mathrm{~atm})$ then pressurized with $\mathrm{H}_{2}(30 \mathrm{~atm})$ and disconnected from the $\mathrm{H}_{2}$ source. Then the autoclave was placed in an oil bath preheated to $60^{\circ} \mathrm{C}$. After 72 hours, the autoclave was cooled in an ice bath, and the pressure was released slowly. The reaction mixture was diluted with DCM and filtered via a short silica gel plug. 48\% conversion of $p$-F-benzaldehyde was obtained (TON: 24000).

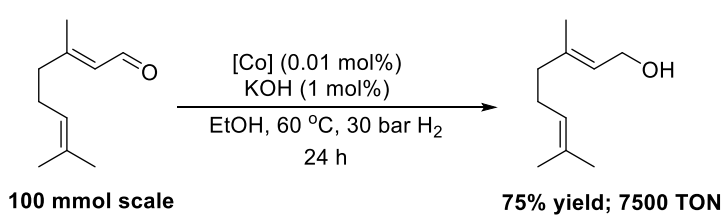

In an argon glovebox, a $50 \mathrm{~mL}$ vial equipped with a magnetic stirring bar, was added $100 \mu \mathrm{L}$ catalyst solution ([Co $\left.{ }^{\mathrm{II}}(\mathbf{L 1})\left(\mathrm{CH}_{3} \mathrm{CN}\right)\right]\left(\mathrm{BF}_{4}\right)_{2}: 0.1 \mathrm{M}$ in $\left.\mathrm{CH}_{3} \mathrm{CN}\right)$, citral $(100 \mathrm{mmol}, 15.2 \mathrm{~g})$, potassium hydroxide $(1 \mathrm{~mol} \%, 11.2 \mathrm{mg})$ and 10 $\mathrm{mL}$ ethanol. Then the mixture was transferred to a stain-less autoclave and then was purged by three cycles of pressurization/venting with $\mathrm{H}_{2}(10 \mathrm{~atm})$ then pressurized with $\mathrm{H}_{2}(30 \mathrm{~atm})$ and disconnected from the $\mathrm{H}_{2}$ source. Then the autoclave was placed in an oil bath preheated to $60{ }^{\circ} \mathrm{C}$. After 24 hours, the autoclave was cooled in an ice bath, and the pressure was released slowly. The reaction mixture was diluted with DCM and filtered via a short silica gel plug. The crude product was analyzed by ${ }^{1} \mathrm{H}$ NMR using 1,2-dibromomethane as the internal standard. 7500 TON for citral hydrogenation was obtained on $100 \mathrm{mmol}$ scale.

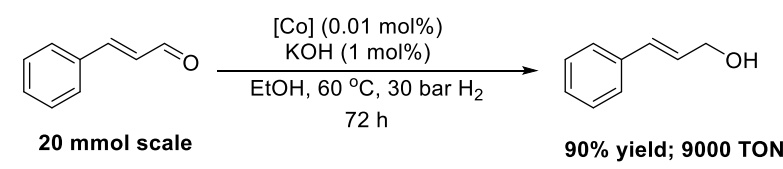

In an argon glovebox, a $50 \mathrm{~mL}$ vial equipped with a magnetic stirring bar, was added $100 \mu \mathrm{L}$ catalyst solution ([Co $\left.{ }^{\mathrm{II}}(\mathbf{L 1})\left(\mathrm{CH}_{3} \mathrm{CN}\right)\right]\left(\mathrm{BF}_{4}\right)_{2}: 0.1 \mathrm{M}$ in $\left.\mathrm{CH}_{3} \mathrm{CN}\right)$, trans-cinnamaldehyde (20 mmol, $2.64 \mathrm{~g}$ ), potassium hydroxide (1 mol\%, $11.2 \mathrm{mg}$ ) and $20 \mathrm{~mL}$ ethanol. Then the mixture was transferred to a stain-less autoclave and then was purged by three cycles of pressurization/venting with $\mathrm{H}_{2}(10 \mathrm{~atm})$ then pressurized with $\mathrm{H}_{2}(30 \mathrm{~atm})$ and disconnected from the $\mathrm{H}_{2}$ source. Then the autoclave was placed in an oil bath preheated to $60{ }^{\circ} \mathrm{C}$. After 72 hours, the autoclave was cooled in an ice bath, and the pressure was released slowly. The reaction mixture was diluted with DCM and filtered via a short silica gel plug. 90\% conversion of trans-cinnamaldehyde was obtained (TON: 9000). 


\section{Moisture sensitivity and TEMPO-inhibited experiments}

Water is a common adventitious impurity in reagents and solvents, and thus we tested the activity of cobalt(II) precatalyst $\left[\mathrm{Co}{ }^{\mathrm{II}}(\mathbf{L} \mathbf{1})\left(\mathrm{CH}_{3} \mathrm{CN}\right)\right]\left(\mathrm{BF}_{4}\right)_{2}(0.1 \mathrm{~mol} \%)$ for the hydrogenation of trans-cinnamaldehyde in the presence of intentionally added water (33 equiv. relative to substrate).

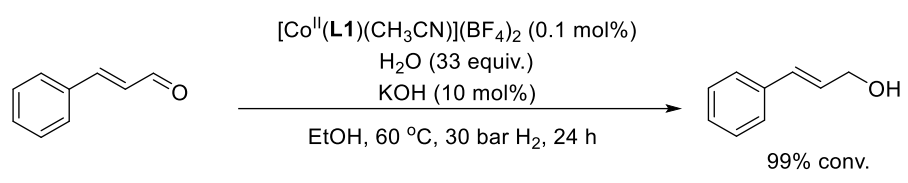

In an argon glovebox, a $5 \mathrm{~mL}$ vial equipped with a magnetic stirring bar, was added $20 \mu \mathrm{L}$ cobalt catalyst solution (0.1 $\mathrm{M}$ in $\left.\mathrm{CH}_{3} \mathrm{CN}\right)$, trans-cinnamaldehyde $(0.2 \mathrm{mmol}, 26.4 \mathrm{mg})$, potassium hydroxide $(10 \mathrm{~mol} \%, 1.12 \mathrm{mg}), 33$ equivalents of water $(6.6 \mathrm{mmol}, 0.12 \mathrm{~mL})$ and $1 \mathrm{~mL}$ ethanol. Then the mixture was transferred to a stain-less autoclave and then was purged by three cycles of pressurization/venting with $\mathrm{H}_{2}(10 \mathrm{~atm})$ then pressurized with $\mathrm{H}_{2}(30 \mathrm{~atm})$ and disconnected from the $\mathrm{H}_{2}$ source. Then the autoclave was placed in an oil bath preheated to $60{ }^{\circ} \mathrm{C}$. After 24 hours, the autoclave was cooled in an ice bath, and the pressure was released slowly. The reaction mixture was diluted with DCM and filtered via a short silica gel plug. The desired (E)-3-phenylprop-2-en-1-ol was offered in quantitative yield indicating remarkable tolerance of moisture in current cobalt catalytic system.

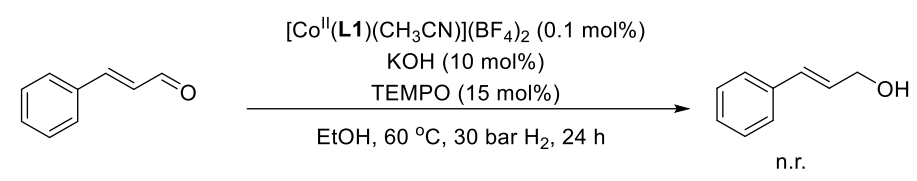

In an argon glovebox, a $5 \mathrm{~mL}$ vial equipped with a magnetic stirring bar, was added $20 \mu \mathrm{L}$ cobalt catalyst solution (0.1 $\mathrm{M}$ in $\left.\mathrm{CH}_{3} \mathrm{CN}\right)$, trans-cinnamaldehyde $(0.2 \mathrm{mmol}, 26.4 \mathrm{mg})$, potassium hydroxide $(10 \mathrm{~mol} \%, 1.12 \mathrm{mg})$, 2,2,6,6-tetramethylpiperidine-1-oxyl (4.86 mg, $15 \mathrm{~mol} \%)$ and $1 \mathrm{~mL}$ ethanol. Then the mixture was transferred to a stain-less autoclave and then was purged by three cycles of pressurization/venting with $\mathrm{H}_{2}(10 \mathrm{~atm})$ then pressurized with $\mathrm{H}_{2}(30 \mathrm{~atm})$ and disconnected from the $\mathrm{H}_{2}$ source. Then the autoclave was placed in an oil bath preheated to $60{ }^{\circ} \mathrm{C}$. After 24 hours, the autoclave was cooled in an ice bath, and the pressure was released slowly. The reaction mixture was diluted with DCM and filtered via a short silica gel plug. Hydrogenation reaction was completely inhibited and trans-cinnamaldehyde was recovered in above $95 \%$ yield. 


\section{Indirect verification of $\mathrm{H}_{2} \mathrm{O}_{2}$ formation}

In an argon glovebox, $\left[\mathrm{Co}{ }^{\mathrm{II}}(\mathbf{L 1})\left(\mathrm{CH}_{3} \mathrm{CN}\right)\right]\left(\mathrm{BF}_{4}\right)_{2}(20 \mathrm{mg}, 0.02 \mathrm{mmol})$ was dissolved in $\mathrm{CD}_{3} \mathrm{CN}(500 \quad \mathrm{~L})$. Then the resulting yellow solution and powder $\mathrm{KOH}(22.4 \mathrm{mg}, 0.4 \mathrm{mmol})$ were transferred to a $\mathrm{J}$. Young tube. After that, $\mathrm{PPh}_{3}$ $(0.6 \mathrm{mg}, 0.002 \mathrm{mmol})$ was added to the above tube and the tube was vortexed for several minutes. The ${ }^{31} \mathrm{P}$ NMR experiments were recorded in specific time intervals. As shown in Figure S2, after $48 \mathrm{~h}$ the clear formation of triphenylphosphine oxide was detected as comparison with the authentic sample. This result shows the possible formation of hydrogen peroxide yielded via the dimerization of $\cdot \mathrm{OH}$. And this provides an evidence for hydroxide ion acting as one-electron reducing agent during the conversion of complex 3 to 4 .

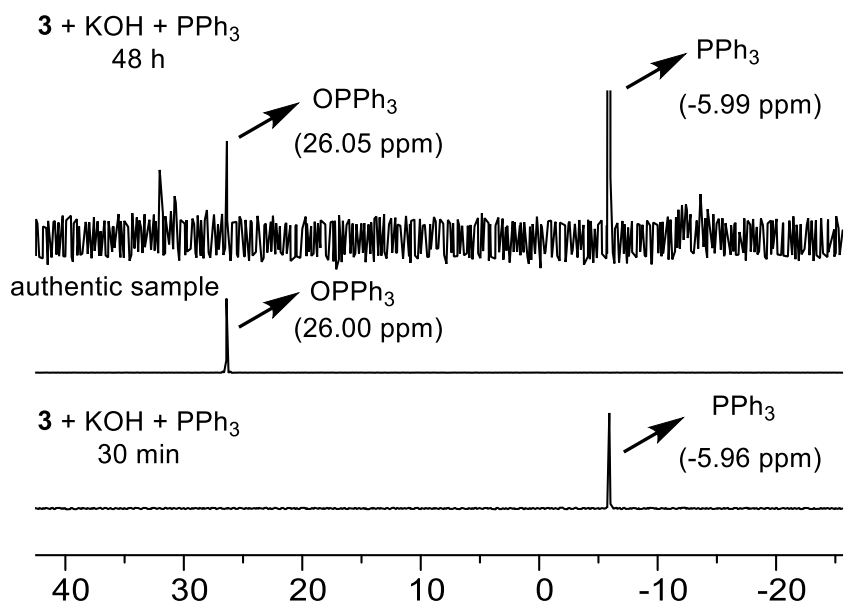

Figure S2. ${ }^{31} \mathrm{P}$ NMR for detecting the formation of triphenylphosphine oxide 


\section{Mechanistic experiments}

\subsection{Procedure for synthesis of $\left[\mathrm{Co}^{\mathrm{I}}(\mathrm{L1})\left(\mathrm{CH}_{3} \mathrm{CN}\right)\right]\left(\mathrm{BF}_{4}\right)$ using $\mathrm{KOH}$ as activator}

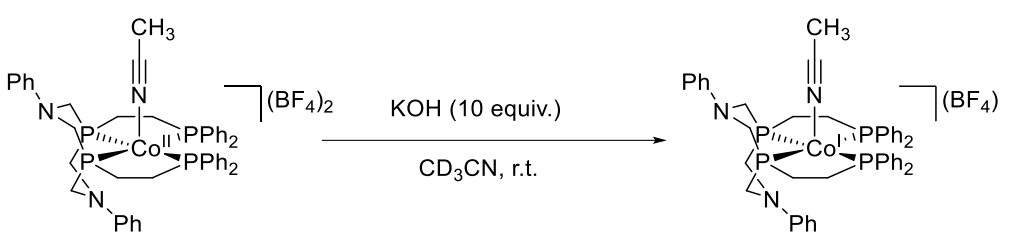

In an argon glovebox, $\left[\mathrm{Co}(\mathbf{I I} \mathbf{L})\left(\mathrm{CH}_{3} \mathrm{CN}\right)\right]\left(\mathrm{BF}_{4}\right)_{2}(10 \mathrm{mg}, 0.01 \mathrm{mmol})$ was dissolved in $\mathrm{CD}_{3} \mathrm{CN}(500 \mu \mathrm{L})$. Then the resulting yellow solution and powder $\mathrm{KOH}(11.2 \mathrm{mg}, 0.2 \mathrm{mmol})$ were transferred to a J. Young tube and the tube was vortexed for several minutes. The ${ }^{1} \mathrm{H}$ and ${ }^{31} \mathrm{P}$ NMR experiments were recorded in specific time intervals. As shown in Figure S3, one diamagnetic cobalt species was gradually formed which was confirmed to be $\left[\mathrm{Co}^{\mathrm{I}}(\mathbf{L} \mathbf{1})\left(\mathrm{CH}_{3} \mathrm{CN}\right)\right]\left(\mathrm{BF}_{4}\right)$ as comparison with the authentic sample prepared using $\mathrm{KC}_{8}$ as the reductant according to the reported literature ${ }^{[1]}$.

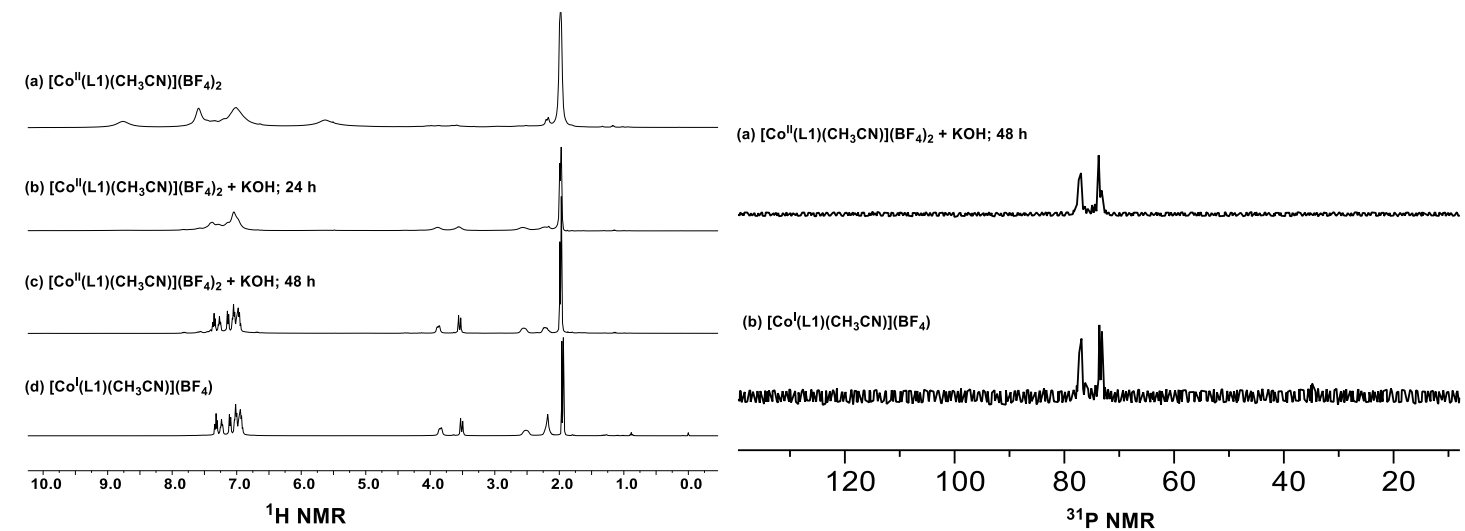

Figure S3. Formation of $\left[\mathrm{Co}^{\mathrm{I}}(\mathrm{L1})\left(\mathrm{CH}_{3} \mathrm{CN}\right)\right]\left(\mathrm{BF}_{4}\right)$ using $\mathrm{KOH}$ as activator monitored by NMR spectroscopy

\subsection{Conversion of $\left[\mathrm{Co}^{\mathrm{I}}(\mathrm{L1})\left(\mathrm{CH}_{3} \mathrm{CN}\right)\right]\left(\mathrm{BF}_{4}\right)$ with $\mathrm{KOH}$}

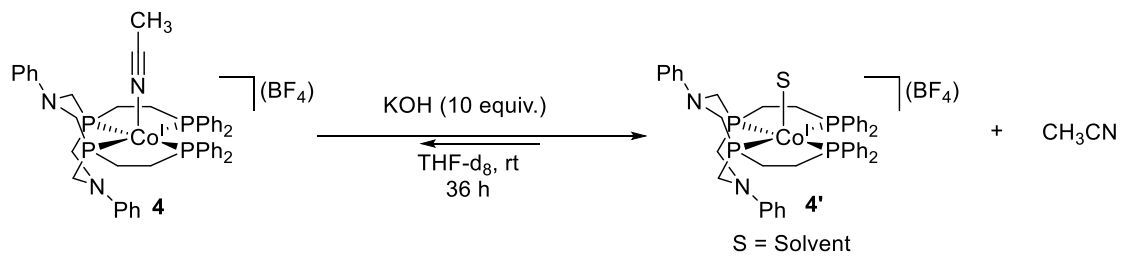

In an argon glovebox, $\left[\mathrm{Co}^{\mathrm{I}}(\mathbf{L 1})\left(\mathrm{CH}_{3} \mathrm{CN}\right)\right]\left(\mathrm{BF}_{4}\right)(9.6 \mathrm{mg}, 0.01 \mathrm{mmol})$ was dissolved in THF- $\mathrm{d}_{8}(500 \mu \mathrm{L})$. Then the resulting deep purple solution and powder $\mathrm{KOH}(5.6 \mathrm{mg}, 0.1 \mathrm{mmol})$ were transferred to a J. Young tube and the tube was sealed tightly and vortexed for several minutes intermittently. Then the mixture was monitored by NMR spectroscopy. The peak with chemical shift at $\delta 1.82 \mathrm{ppm}\left(\mathrm{THF}_{\mathrm{f}} \mathrm{d}_{8}\right)$ was ascribed to the methyl group of acetonitrile in $\left[\mathrm{Co}^{\mathrm{I}}(\mathbf{L 1})\left(\mathrm{CH}_{3} \mathrm{CN}\right)\right]\left(\mathrm{BF}_{4}\right)$ complex. During a course of $36 \mathrm{~h}$, the peak was downshifted to $1.94 \mathrm{ppm}$ as shown in Figure S4. The peak intensity at $\delta 1.94 \mathrm{ppm}$ was increased via adding 10 equiv. of $\mathrm{CH}_{3} \mathrm{CN}$, indicating that the peak was ascribed to the dissociated $\mathrm{CH}_{3} \mathrm{CN}$ molecule and no recoordination of $\mathrm{CH}_{3} \mathrm{CN}$ to cobalt center was observed. Therefore, a coordinatively unsaturated and stable $16 \mathrm{e}^{-}$cobalt(I) species $\mathbf{4}^{\prime}$ possibly formed. ${ }^{31} \mathrm{P}$ NMR spectroscopy of $4^{\prime}$ presented two broad peaks at $76.5 \mathrm{ppm}$ and $73.5 \mathrm{ppm}$, respectively, which are similar to the resonances of cobalt(I) complex 4. Then, the above-mentioned J. Young tube was immersed in liquid nitrogen and evacuated its head regions and refilled with dihydrogen via vacuum line. The whole process was repeated for three times. After that, the formation of 
trans- $\left[(\mathrm{H})_{2}-\mathrm{Co}^{\mathrm{III}}(\mathbf{L} \mathbf{1})\right]\left(\mathrm{BF}_{4}\right)$ was observed along with a little amount of $\left[\mathrm{Co}^{\mathrm{I}}(\mathbf{L} \mathbf{1})-\mathrm{H}\right]$.

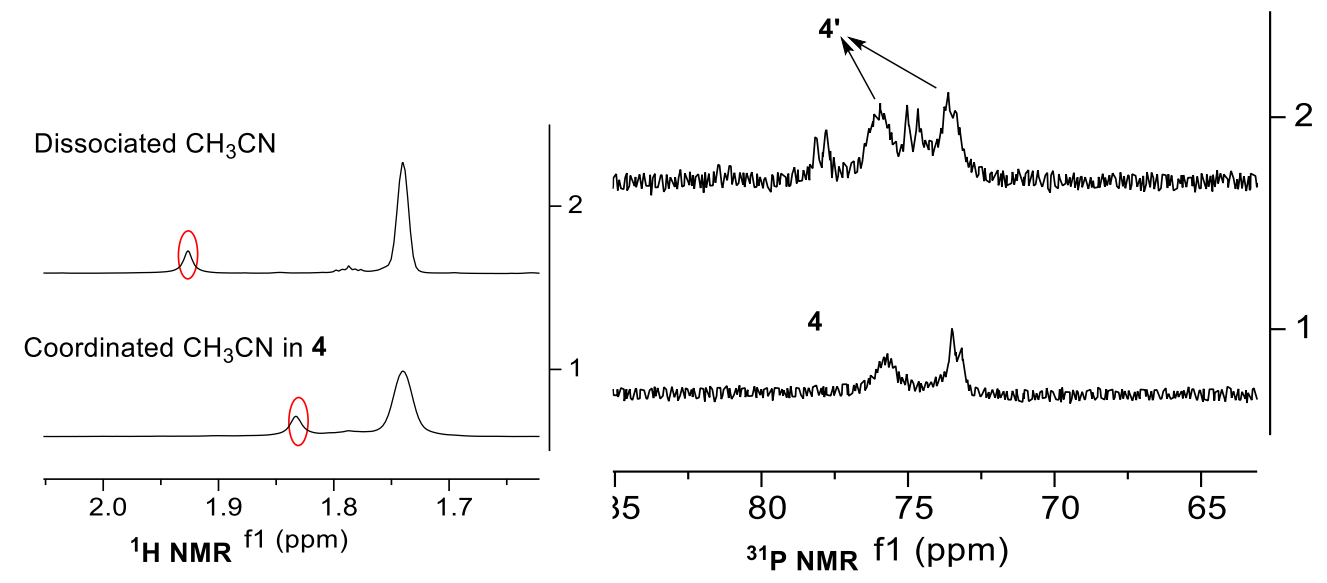

Figure S4. Inorganic base benefited to acetonitrile dissociation

\subsection{Reaction of $\left[\mathrm{Co}^{\mathrm{I}}(\mathrm{L1})\left(\mathrm{CH}_{3} \mathrm{CN}\right)\right]\left(\mathrm{BF}_{4}\right)$ with dihydrogen in the presence of $\mathrm{KOH}$}

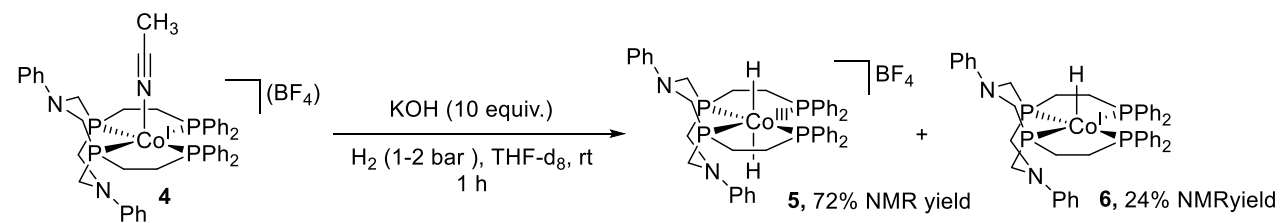

In an argon glovebox, $\left[\mathrm{Co}^{\mathrm{I}}(\mathbf{L 1})\left(\mathrm{CH}_{3} \mathrm{CN}\right)\right]\left(\mathrm{BF}_{4}\right)(9.6 \mathrm{mg}, 0.01 \mathrm{mmol})$ was dissolved in THF-d $\mathrm{d}_{8}(500 \mu \mathrm{L})$. Then the resulting deep purple solution and powder $\mathrm{KOH}(5.6 \mathrm{mg}, 0.1 \mathrm{mmol})$ were transferred to a J. Young tube and the tube was immersed in liquid nitrogen and evacuated its head regions and refilled with dihydrogen via vacuum line. The whole process was repeated for three times. After that the tube was gradually warmed to room temperature and vortexed for several minutes. The ${ }^{1} \mathrm{H}$ and ${ }^{31} \mathrm{P}$ NMR were recorded as shown in Figure S5. And the formation of trans-[(H) $\left.{ }_{2}-\mathrm{Co}^{\mathrm{III}}(\mathbf{L 1})\right]\left(\mathrm{BF}_{4}\right) 5$ and $\left[\mathrm{Co}^{\mathrm{I}}(\mathrm{H})(\mathbf{L 1})\right] 6$ was observed (72\% and 24\% NMR yields, respectively). 

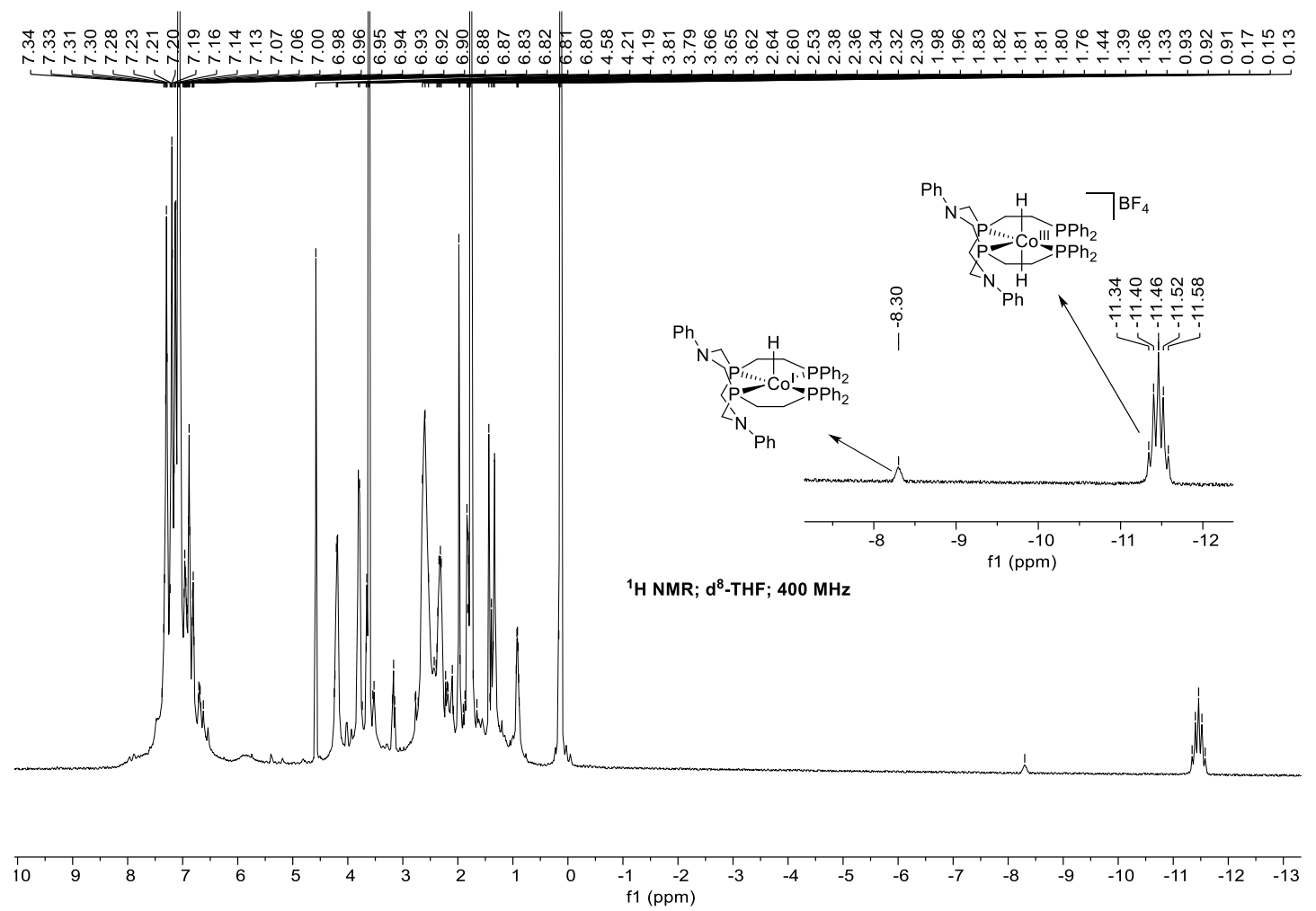

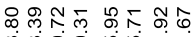

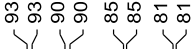

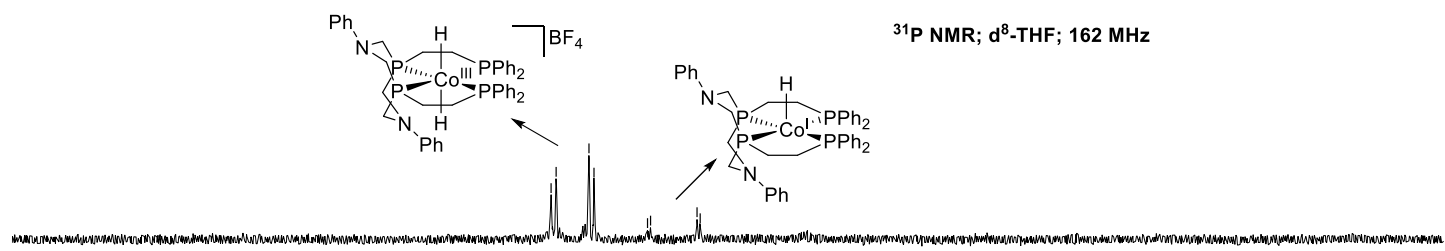

$\begin{array}{lllllllllllllllllllllllllll}135 & 130 & 125 & 120 & 115 & 110 & 105 & 100 & 95 & 90 & 85 & 80 & 75 & 70 & 65 & 60 & 55 & 50 & 45 & 40 & 35 & 30 & 25\end{array}$

Figure S5. $\left[\mathrm{Co}^{\mathrm{I}}(\mathrm{H})(\mathrm{L1})\right]$ and trans $-\left[(\mathrm{H})_{2}-\mathrm{Co}^{\mathrm{III}}(\mathrm{L1})\right]\left(\mathrm{BF}_{4}\right)$ formation monitored by NMR spectroscopy 


\subsection{Reaction of $\left[\mathrm{Co}^{\mathrm{II}}(\mathrm{L1})\left(\mathrm{CH}_{3} \mathrm{CN}\right)\right]\left(\mathrm{BF}_{4}\right)_{2}$ with dihydrogen in the presence of $\mathrm{KOH}$}

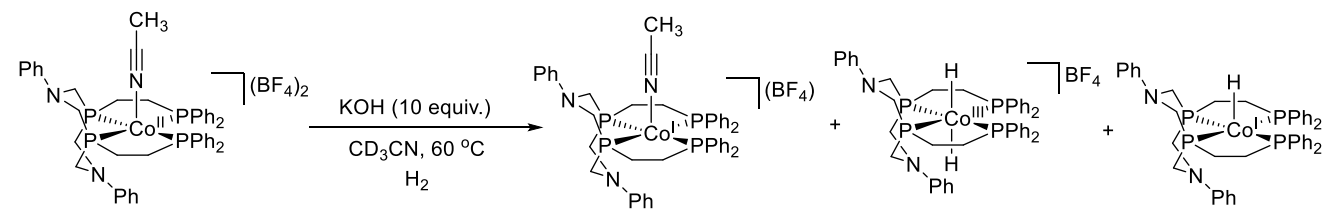

In an argon glovebox, $\left[\mathrm{Co}{ }^{\mathrm{II}}(\mathbf{L 1})\left(\mathrm{CH}_{3} \mathrm{CN}\right)\right]\left(\mathrm{BF}_{4}\right)_{2}(10.0 \mathrm{mg}, 0.01 \mathrm{mmol})$ was dissolved in $\mathrm{CD}_{3} \mathrm{CN}(500 \mu \mathrm{L})$. Then the resulting orange solution and powder $\mathrm{KOH}(5.6 \mathrm{mg}, 0.1 \mathrm{mmol})$ were transferred to a J. Young tube and the tube was immersed in liquid nitrogen and evacuated its head regions and refilled with dihydrogen via vacuum line. The whole process was repeated for three times. Then, the tube was gradually warmed to room temperature and vortexed for several minutes. Finally, the tube was immersed in $60{ }^{\circ} \mathrm{C}$ oil bath for $36 \mathrm{~h}$. The ${ }^{1} \mathrm{H}$ and ${ }^{31} \mathrm{P}$ NMR were recorded as shown in Figure S6 indicating that $\left[\mathrm{Co}^{\mathrm{I}}(\mathbf{L} \mathbf{1})\left(\mathrm{CH}_{3} \mathrm{CN}\right)\right]\left(\mathrm{BF}_{4}\right),\left[\mathrm{Co}^{\mathrm{I}}(\mathrm{H})(\mathbf{L} \mathbf{1})\right]$ and trans- $\left[(\mathrm{H})_{2}-\mathrm{Co}^{\mathrm{III}}(\mathbf{L} \mathbf{1})\right]\left(\mathrm{BF}_{4}\right)$ were formed in the presence of $\left[\mathrm{Co}^{\mathrm{II}}(\mathbf{L} \mathbf{1})\left(\mathrm{CH}_{3} \mathrm{CN}\right)\right]\left(\mathrm{BF}_{4}\right)_{2}$ complex, $\mathrm{KOH}$ and $\mathrm{H}_{2}$.

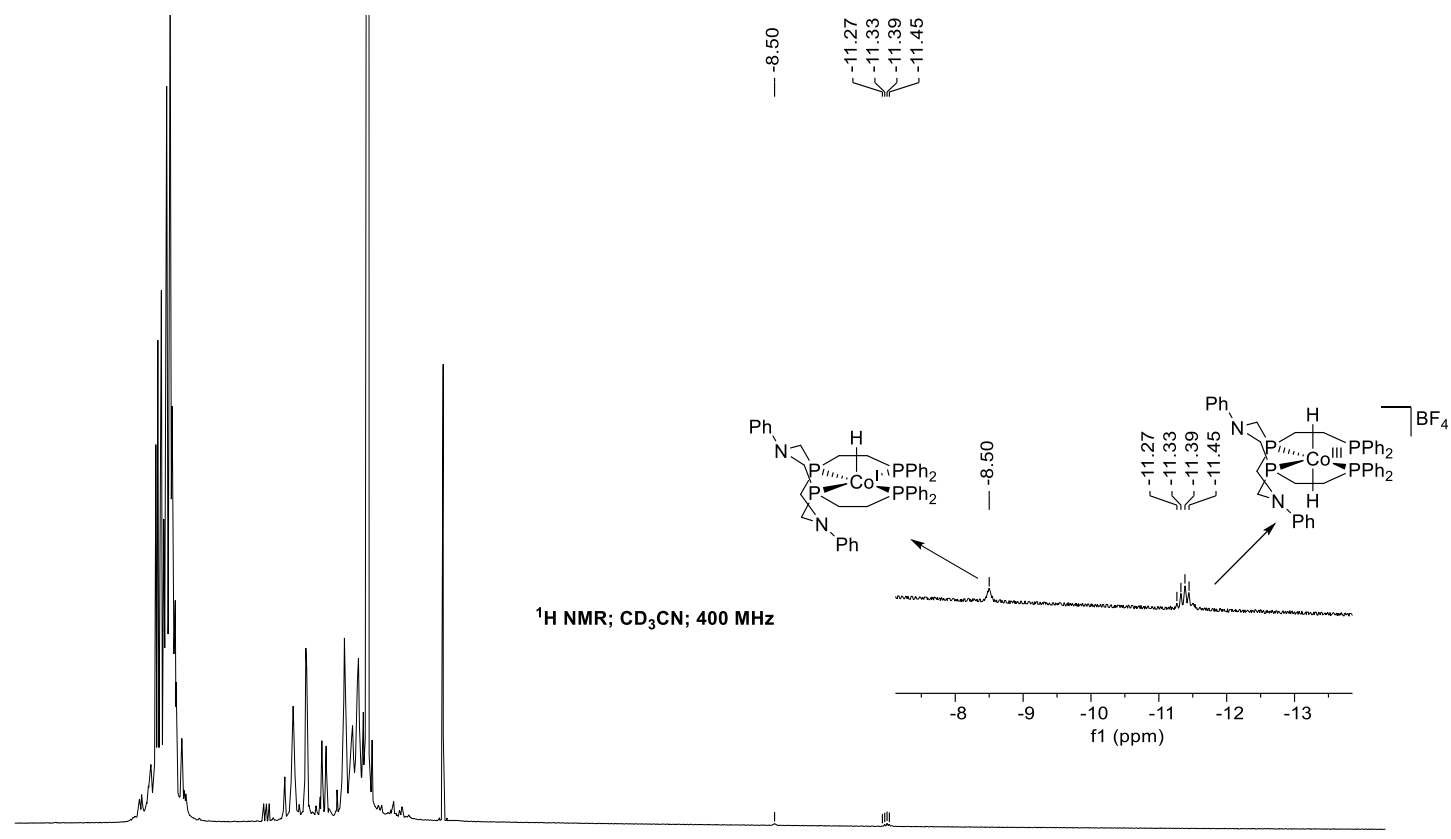

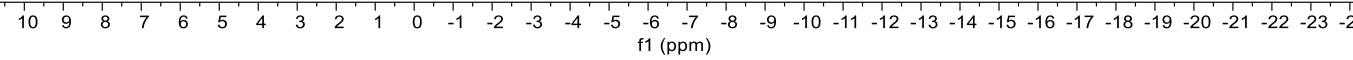




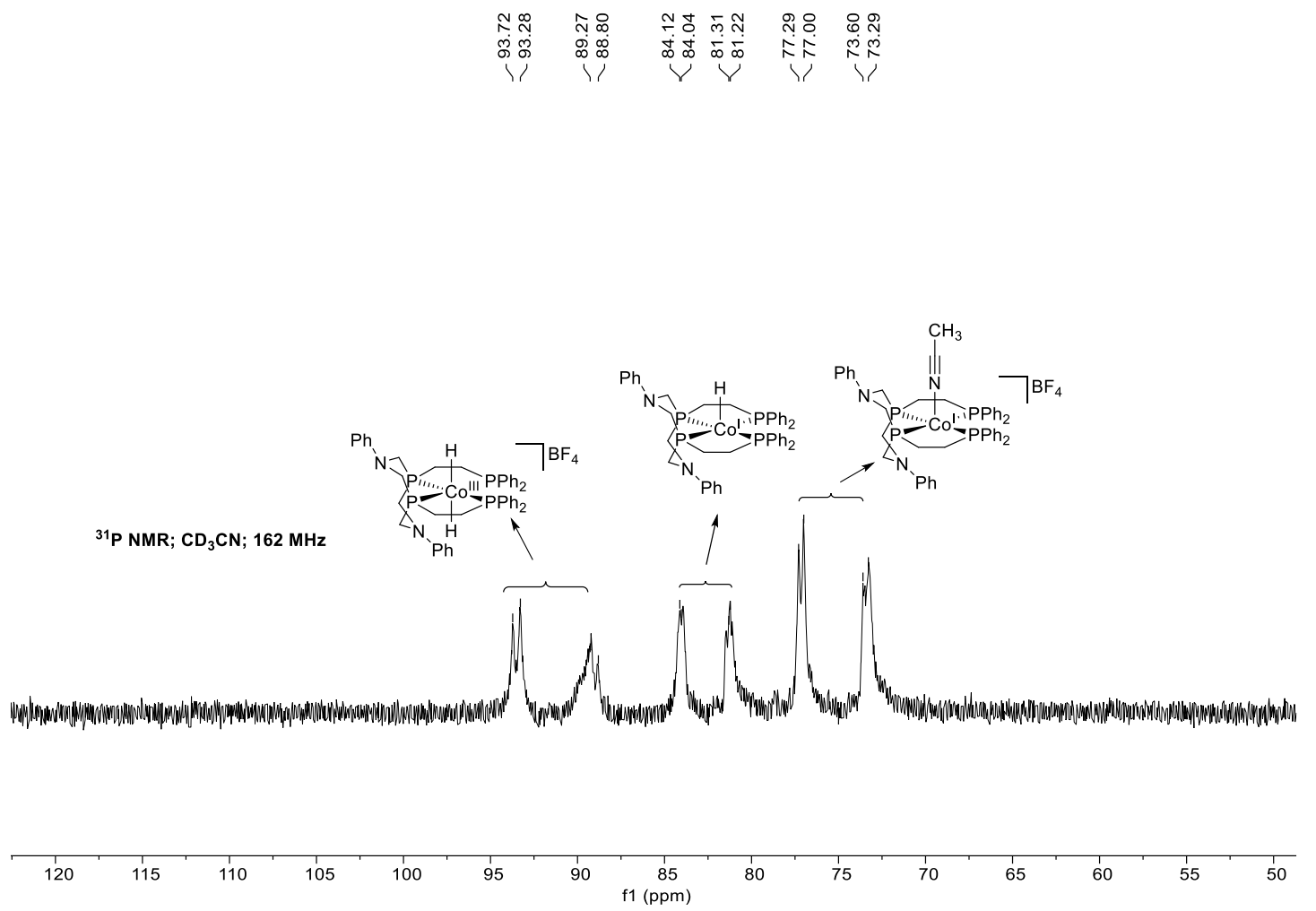

Figure S6. NMR spectroscopy for reaction of $\left[\mathrm{Co}^{\mathrm{II}}(\mathrm{L1})\left(\mathrm{CH}_{3} \mathrm{CN}\right)\right]\left(\mathrm{BF}_{4}\right)_{2}$ with dihydrogen in the presence of $\mathrm{KOH}$

\subsection{Procedure for stoichiometric reaction of cobalt hydride species with trans-cinnamaldehyde and quinoline}

\section{1) Stoichiometric reaction of cobalt hydride 5 and 6 with trans-cinnamaldehyde}

Cobalt hydride species $\mathbf{5}$ and $\mathbf{6}$ were prepared independently according to the procedure described above. In an argon glovebox, a $10 \mathrm{~mL}$ shrek tube equipped with a magnetic stirring bar was added $0.05 \mathrm{mmol}$ cobalt(I) hydride 6 in $0.1 \mathrm{~mL}$ THF, trans-cinnamaldehyde $(0.05 \mathrm{mmol}, 6.5 \mu \mathrm{L})$ and $0.5 \mathrm{~mL}$ EtOH. Then the tube was sealed tightly and placed in an oil bath preheated to $60{ }^{\circ} \mathrm{C}$. After $24 \mathrm{~h}$, the reaction mixture was cooled down and diluted with DCM and filtered via a short silica gel plug. $98 \%$ yield of the alcohol product was offered $(6.6 \mathrm{mg})$.

The procedure for stoichiometric reaction of complex $\mathbf{5}$ with trans-cinnamaldehyde was similar to that for complex $\mathbf{6}$. And no reaction occurred when complex 5 reacts with trans-cinnamaldehyde. But when 10 equiv. KOH was incorporated into such reaction, $98 \%$ conversion of trans-cinnamaldehyde was observed.

\section{2) Stoichiometric reaction of cobalt hydride 6 with quinoline}

In an argon glovebox, a $10 \mathrm{~mL}$ shrek tube equipped with a magnetic stirring bar was added $0.13 \mathrm{mmol}$ cobalt(I) hydride 6 in $0.25 \mathrm{~mL}$ THF, quinoline $(0.05 \mathrm{mmol}, 6.0 \mu \mathrm{L})$ and $0.25 \mathrm{~mL} i \operatorname{PrOH}$. Then the tube was sealed tightly and placed in an oil bath preheated to $100{ }^{\circ} \mathrm{C}$. After $48 \mathrm{~h}$, the reaction mixture was cooled down and diluted with DCM and filtered via a short silica gel plug. $95 \%$ yield of the tetrahydroquinoline was obtained $(6.2 \mathrm{mg})$.

9.6 Procedure for reaction of trans- $\left[(\mathrm{H})_{2}-\mathrm{Co}^{\mathrm{III}}(\mathrm{L1})\right]\left(\mathrm{BF}_{4}\right)$ with base

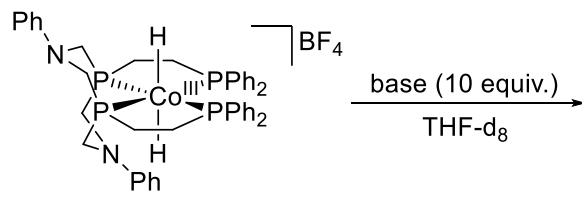

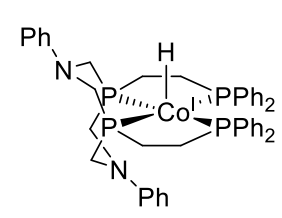

DBU, $60^{\circ} \mathrm{C}$ : n.r.

tBuTMG, $60^{\circ} \mathrm{C}:$ n.r.

$\mathrm{KOH}$, r.t.: $82 \%$ conv. $24 \mathrm{~h}$.

tBuOK, r.t.: quant. within $10 \mathrm{~min}$

EtOK, r.t.: quant. within $10 \mathrm{~min}$ 
The J. Young tube containing the orange solution of trans $-\left[(\mathrm{H})_{2}-\mathrm{Co}^{\mathrm{III}}(\mathbf{L 1})\right]\left(\mathrm{BF}_{4}\right)$ in $\mathrm{THF}-\mathrm{d}_{8}(0.01 \mathrm{mmol}$ in $500 \mu \mathrm{L}$ THF- $\mathrm{d}_{8}$ ) was transferred to the glovebox. Then the corresponding organic or inorganic base (10 equiv.) was transferred to the J. Young tube and the tube was sealed tightly and vortexed for several minutes. Then, ${ }^{1} \mathrm{H}$ and ${ }^{31} \mathrm{P}$ NMR were recorded in specific time intervals. The results showed that organic base like DBU and $t$ BuTMG could not deprotonate trans- $\left[(\mathrm{H})_{2}-\mathrm{Co}^{\mathrm{III}}(\mathbf{L 1})\right]\left(\mathrm{BF}_{4}\right)$ to form $\left[\mathrm{Co}^{\mathrm{I}}(\mathrm{H})(\mathbf{L 1})\right]$ even at $60{ }^{\circ} \mathrm{C}$. Inorganic base like $\mathrm{KOH}$, $t \mathrm{BuOK}$ and EtOK deprotonated trans- $\left[(\mathrm{H})_{2}-\mathrm{Co}^{\mathrm{III}}(\mathbf{L 1})\right]\left(\mathrm{BF}_{4}\right)$ to form $\left[\mathrm{Co}^{\mathrm{I}}(\mathrm{H})(\mathbf{L 1})\right]$ with different efficiency at room temperature $(\mathrm{KOH}: 82 \%$ conv. within $24 \mathrm{~h}$; $t \mathrm{BuOK}$ and EtOK: complete conv. within $10 \mathrm{~min}$ ).

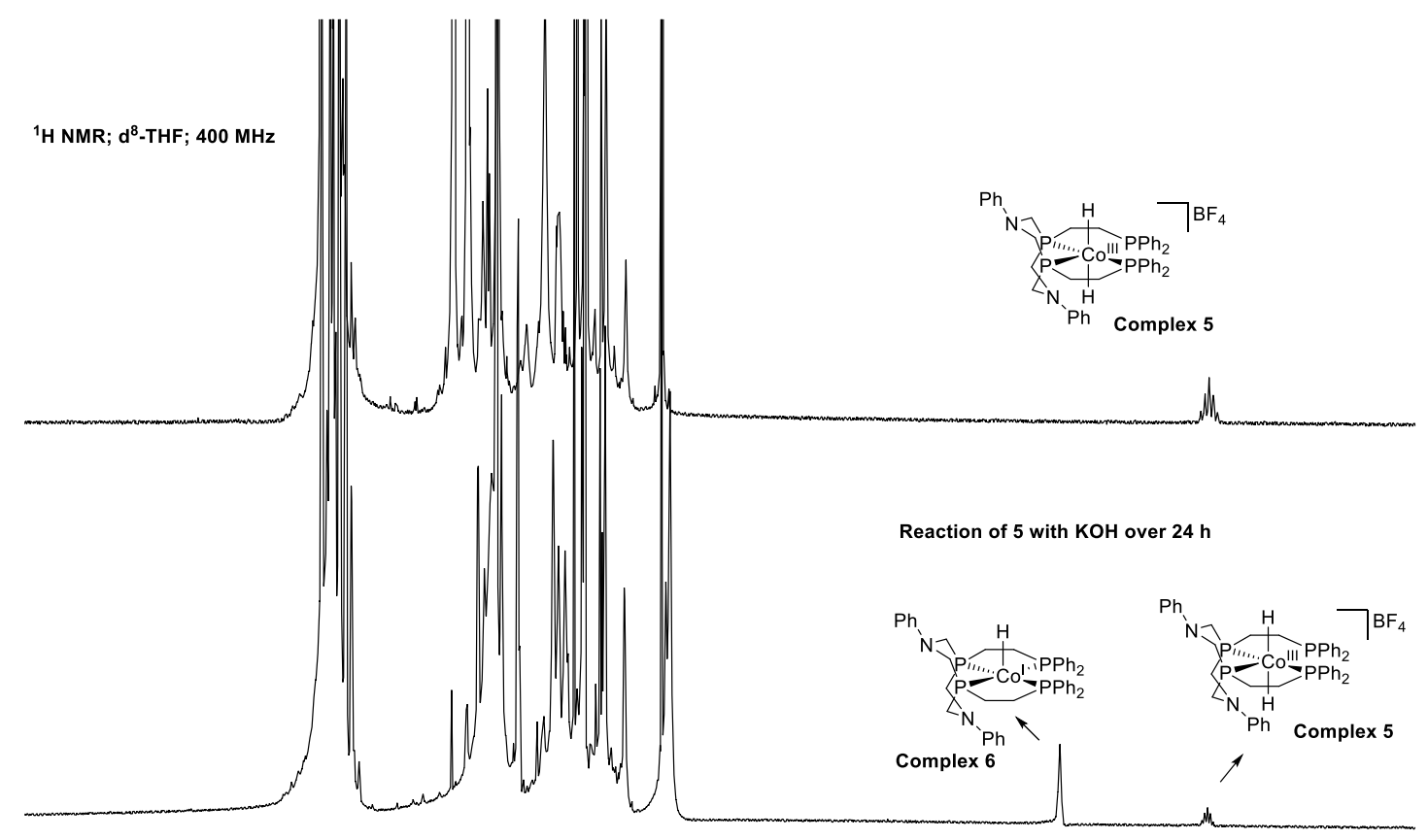

$\begin{array}{llllllllllllllllllllllllllllll}13 & 12 & 11 & 10 & 9 & 8 & 7 & 6 & 5 & 4 & 3 & 2 & 1 & 0 & -1 & -2 & -3 & -4 & -5 & -6 & -7 & -8 & -9 & -10 & -11 & -12 & -13 & -14 & -15\end{array}$ 


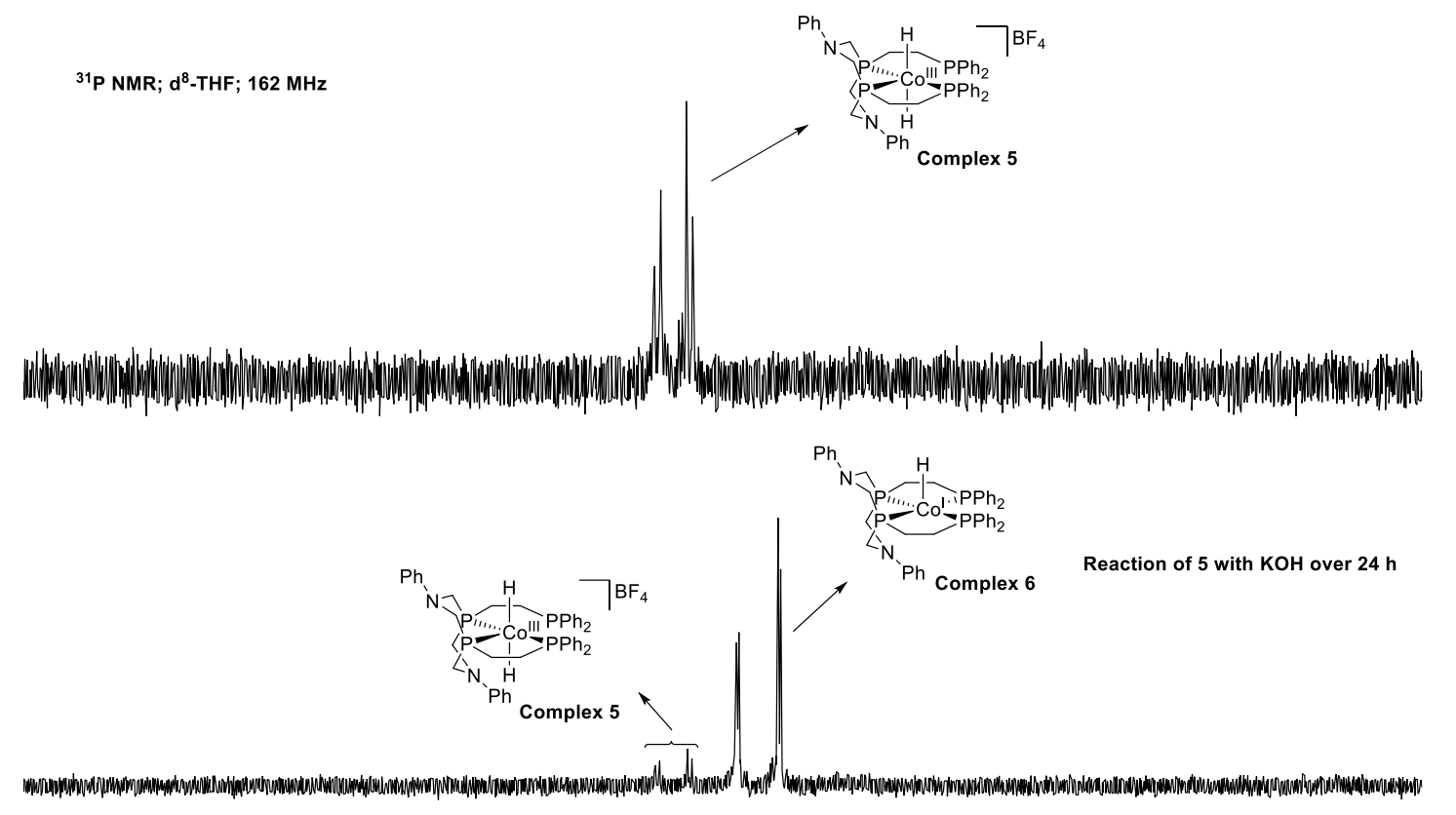

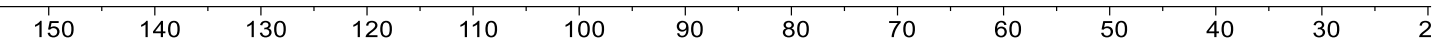

Figure S7. NMR spectroscopy for reaction of trans-[(H) $\left.)_{2}-\mathrm{Co}^{\mathrm{III}}(\mathrm{L1} 1)\right]\left(\mathrm{BF}_{4}\right)$ with $\mathrm{KOH}$

\subsection{Reaction of $\left[\mathrm{Co}^{\mathrm{I}}(\mathrm{H})(\mathrm{L1})\right]$ with 4-F-benzaldehyde}

The J. Young tube containing the solution of $0.01 \mathrm{mmol}\left[\mathrm{Co}^{\mathrm{I}}(\mathrm{H})(\mathbf{L 1})\right]$ in $500 \mu \mathrm{L}$ THF- $\mathrm{d}_{8}$ was transferred to the glovebox. Then 1.2 equiv. 4-F-benzaldehyde $(0.012 \mathrm{mmol}, 1.2 \mu \mathrm{L})$ was added to the above solution via microsyringe and the tube was sealed tightly and vortexed for several minutes. Then, ${ }^{1} \mathrm{H},{ }^{31} \mathrm{P}$ and ${ }^{19} \mathrm{~F}$ NMR were recorded. The results showed that $\left[\mathrm{Co}^{\mathrm{I}}(\mathrm{H})(\mathbf{L 1})\right]$ reacts with 4-F-benzaldehyde to yield the (4-fluorophenyl)methanol along with the regeneration of cobalt(I) complex $\mathbf{4}^{\prime}$.
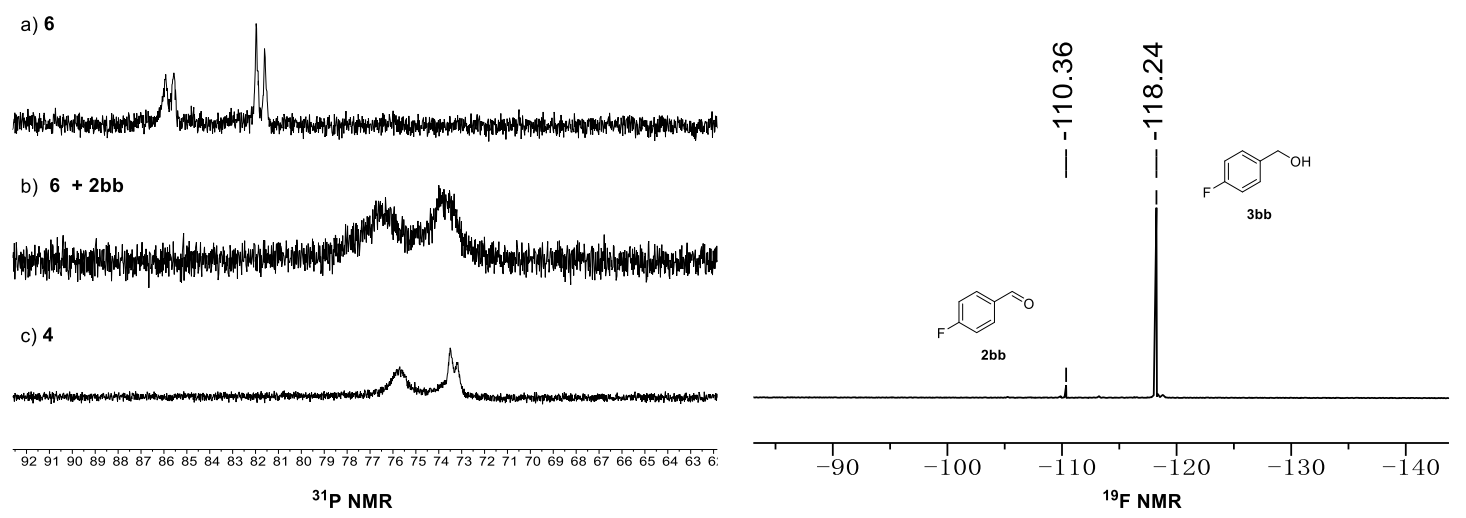

Figure S8. NMR spectroscopy for reaction of $\left[\mathrm{Co}^{\mathrm{I}}(\mathrm{H})(\mathrm{L1})\right]$ with 4-F-benzaldehyde

9.8 Hydrogenation of trans-cinnamaldehyde using $D_{2}$ and cobalt catalyst 


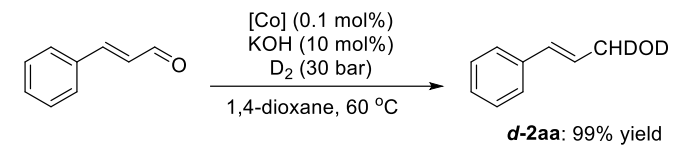

In an argon glovebox, a $5 \mathrm{~mL}$ vial equipped with a magnetic stirring bar, was added $20 \mu \mathrm{L}$ cobalt catalyst solution $(0.1 \mathrm{M}$ in $\left.\mathrm{CH}_{3} \mathrm{CN}\right)$, trans-cinnamaldehyde ( $\left.0.2 \mathrm{mmol}, 26.4 \mathrm{mg}\right)$, potassium hydroxide $(10 \mathrm{~mol} \%, 1.12 \mathrm{mg}$ ) and $1 \mathrm{~mL}$ 1,4-dioxane. Then the mixture was transferred to a stain-less autoclave and then was purged by three cycles of pressurization/venting with $\mathrm{D}_{2}(10 \mathrm{~atm})$ then pressurized with $\mathrm{D}_{2}(30 \mathrm{~atm})$ and disconnected from the $\mathrm{D}_{2}$ source. Then the autoclave was placed in an oil bath preheated to $60^{\circ} \mathrm{C}$. After 24 hours, the autoclave was cooled in an ice bath, and the pressure was released slowly. The reaction mixture was diluted with DCM and filtered via a short silica gel plug. The desired $\boldsymbol{d}$-2aa was offered in quantitative yield. ${ }^{1} \mathrm{H}$ NMR $(400 \mathrm{MHz}$, Chloroform-d) $\delta 7.42-7.36(\mathrm{~m}, 2 \mathrm{H}), 7.35-7.28(\mathrm{~m}, 2 \mathrm{H}), 7.28-7.21$ $(\mathrm{m}, 1 \mathrm{H}), 6.61(\mathrm{dd}, \mathrm{J}=15.9,1.5 \mathrm{~Hz}, 1 \mathrm{H}), 6.44-6.26(\mathrm{~m}, 1 \mathrm{H}), 4.30(\mathrm{dt}, \mathrm{J}=5.8,1.9 \mathrm{~Hz}, 1 \mathrm{H}) ;{ }^{13} \mathrm{C} \mathrm{NMR}(101 \mathrm{MHz}$, Chloroform- $d$ ) $\delta 136.7,131.3,128.6,128.4,127.8,126.5,63.4(\mathrm{t}, \mathrm{J}=21.9 \mathrm{~Hz}, 1 \mathrm{C})$. The spectroscopic data were in agreement with the reported data ${ }^{[3]}$. 


\section{Computational studies}

DFT calculations were performed using the Gaussian 16 package ${ }^{[4]}$. All of the structures are optimized using the $\omega \mathrm{B} 97 \mathrm{XD}$ functional ${ }^{[5]}$ with the basis set SDD for $\mathrm{Co}^{[6]}, 6-31+\mathrm{G}^{*}$ for $\mathrm{N}, \mathrm{O}^{[7]}$ and $6-31 \mathrm{G}^{*}$ for other main-group atoms ${ }^{[7 a, 8]}$. Vibrational analyses were performed to ensure the transition state structures to have only one imaginary frequency. Transition state structures were confirmed to connect appropriate reactants or products by intrinsic reaction coordinate (IRC) calculations ${ }^{[9]}$. Solvent effects in ethanol were considered using the SMD model ${ }^{[10]}$ at the $\omega \mathrm{B} 97 \mathrm{XD} / 6-311++\mathrm{G}^{* *}$ level ${ }^{[5,11]}$ with SDD for Co. The 3D molecular structures were generated by using CYL-view ${ }^{[12]}$.

Table S3. Summary of transition states in cobalt catalyzed hydrogenation of trans-cinnamaldehyde.

\begin{tabular}{cc}
\hline Transition state & $\Delta \Delta \mathrm{G}^{\ddagger}(\mathrm{kcal} / \mathrm{mol})$ \\
\hline $\mathbf{1 , 2}-\mathrm{TS1}$ & 0 \\
$\mathbf{1 , 2}-\mathrm{TS2}$ & 3.38 \\
$\mathbf{1 , 2}-\mathrm{TS3}$ & 2.31 \\
\hline $\mathbf{1 , 4 - T S 1}$ & 3.53 \\
$\mathbf{1 , 4 - T S 2}$ & 4.41 \\
\hline
\end{tabular}

Thermochemical data and coordinates of transition states of 1,2-reduction and 1,4-reduction of trans-cinnamaldehyde. 


\section{1,2-TS1}

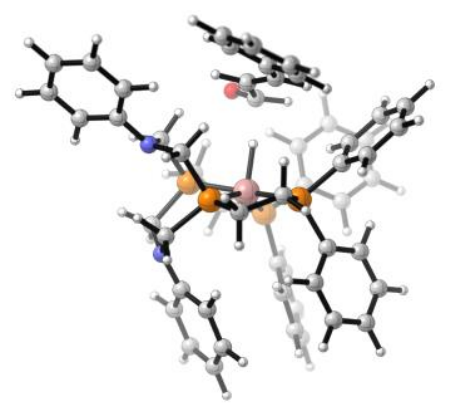

Imaginary Freq $=1$

Temperature $=298.15$ Kelvin

Pressure $=1 \mathrm{~atm}$

Frequencies scaled by $=1$

Electronic Energy $(\mathrm{EE})=-3747.9269$ Hartree

Zero-point Energy Correction $=0.960709$ Hartree

Thermal Correction to Energy = 1.015383 Hartree

Thermal Correction to Enthalpy $=1.016327$ Hartree

Thermal Correction to Free Energy $=0.872268$ Hartree

$\mathrm{EE}+$ Zero-point Energy $=-3746.9662$ Hartree

$\mathrm{EE}+$ Thermal Energy Correction $=-3746.9115$ Hartree

$\mathrm{EE}+$ Thermal Enthalpy Correction $=-3746.9106$ Hartree

$\mathrm{EE}+$ Thermal Free Energy Correction $=-3747.0546$ Hartree

$\mathrm{E}($ Thermal $)=637.162 \mathrm{kcal} / \mathrm{mol}$

Heat Capacity $(\mathrm{Cv})=221.079 \mathrm{cal} / \mathrm{mol}-\mathrm{kelvin}$

Entropy $(\mathrm{S})=303.197 \mathrm{cal} / \mathrm{mol}-\mathrm{kelvin}$

Co $-0.16078600-0.253610000 .24130300$

N $3.08965100-2.196354000 .32626400$

N -0.19670100 -3.60243900 -0.78466900

P - $1.96073800-0.335758001 .50664500$

P $0.53115800-1.932990001 .34482100$

P $1.05641400-1.16460900-1.23009400$

P - $0.496215001 .35599100-1.17792500$

C -1.383971001 .561955003 .49247100$

H -0.529565000 .933232003 .73428600$

C -1.525829002 .778513004 .15475000$

H -0.809991003 .050433004 .92496900$

C -2.565232003 .644094003 .82000500$

H - 2.671180004 .595985004 .33270900

C -3.467795003 .281705002 .82354700$

H -4.281785003 .949571002 .55602100$

C -3.333706002 .061032002 .16681000$

H -4.04594600 1.788391001 .39344700

C -2.287425001 .190096002 .48952300$

C - $3.97657600-0.91421700-0.34964900$

H - $3.18379600-0.82979800-1.08445300$

C -5.28398100 - $1.17725100-0.75645500$
H - 5.49995300 - $1.27774800-1.81495600$

C $-6.29409300-1.316013000 .18636900$

H -7.31241700 - $1.52402200-0.13033400$

C $-5.99894300-1.188789001 .54488000$

H -6.78474700 -1.29865200 2.28695200

C $-4.70062300-0.906007001 .94756600$

H $-4.48486400-0.773056003 .00482200$

C $-3.67597400-0.762868001 .00038100$

C $-1.68440000-1.616523002 .86033700$

H - $2.13079600-1.287259003 .80385500$

H - $2.22586500-2.513454002 .53544600$

C $-0.19283500-1.925969003 .02982900$

H $-0.02515200-2.866657003 .56735900$

H $0.33734000-1.121095003 .55650300$

C $2.36020200-2.015563001 .59008100$

H $2.64345100-1.109018002 .14135000$

H $2.58061600-2.869393002 .23670200$

C $5.51672100-1.847114000 .01934900$

H $5.34199800-0.89694100-0.47411400$

C $6.82565400-2.296629000 .18405800$

H $7.64449000-1.68921700-0.19144300$

C $7.08803600-3.497801000 .83076400$

H $8.10909200-3.842065000 .96164300$

C $6.01997300-4.259548001 .30357800$

H $6.20482600-5.209224001 .79732900$

C $4.71339600-3.825815001 .12976300$

H $3.88914400-4.446128001 .47054500$

C $4.44045200-2.606187000 .49305000$

C $2.85777600-1.22999000-0.74226900$

H $3.44132500-1.56302200-1.60825500$

H $3.16282600-0.20279600-0.49629300$

C $0.82921800-3.00073100-1.60818300$

H $0.61581700-3.16863900-2.66545000$

H $1.77551700-3.50362200-1.37906500$

C - $1.92102400-2.99356800-2.41295400$

H $-1.22659900-2.36955900-2.96113000$

C $-3.21061000-3.14192700-2.91114700$

H $-3.47156200-2.64698200-3.84276600$

C $-4.16268800-3.88355200-2.22338800$

H $-5.17094700-3.99111300-2.60959600$

C $-3.80009500-4.46434200-1.01136100$

H $-4.52819600-5.03613100-0.44345700$

C $-2.51049800-4.34066700-0.51417300$

H $-2.26208700-4.855596000 .40736500$

C $-1.52786600-3.61609100-1.21497700$

C $0.14997100-3.636584000 .62373600$

H $1.02430900-4.286318000 .76493200$

H - $0.66935300-4.057643001 .20565400$

C $1.11553000-0.26038200-2.84706800$

H $2.09636100-0.34114500-3.32884600$ 
H $0.38521800-0.73208600-3.51413300$ C $0.751947001 .20127300-2.56960500$ H $0.403275001 .70336500-3.47817900$ H $1.642184001 .73377600-2.21336100$ C - $2.383529000 .66059000-3.17360400$ H - $1.606032000 .03342700-3.59885200$ C -3.65059700 $0.64654300-3.74993000$ H -3.83903300 $0.02708700-4.62234200$ C -4.67212700 $1.42210700-3.20881100$ H -5.66327900 1.40655500 -3.65217800 C -4.41483300 2.21805600-2.09594800 H -5.20568800 2.82635200 -1.66710400 C -3.14606000 2.23714600-1.52642800 H -2.95786800 $2.86496200-0.65997100$ C -2.11491400 $1.45365400-2.05459700$ C -0.493944003 .515384000 .60118000$ H -0.78979900 2.773782001 .33154000 C -0.320682004 .841167000 .99255600$ H -0.49774400 5.116057002 .02772600 C 0.086790005 .792837000 .06398600 H 0.236626006 .824783000 .36751700 C $0.297610005 .41794600-1.26248600$ H $0.604937006 .15819100-1.99570100$ C $0.110291004 .09566500-1.65282700$ H $0.266871003 .82693100-2.69333000$ C $-0.271688003 .12466100-0.71905600$ H 0.769468000 .621711001 .26574200 C $6.259339002 .01612600-1.32751200$ C $6.046811002 .82881800-2.44057100$ C $4.868439003 .56426300-2.53406500$ C $3.907238003 .47697400-1.53157100$ C $4.096150002 .64574800-0.41861600$ C $5.299516001 .92921900-0.32626800$ C 3.015075002 .520789000 .56885200 C 2.867657001 .510350001 .43375300 C 1.692852001 .340419002 .33884600 O 1.792643000 .627223003 .37272300 H $7.184692001 .45426600-1.23309200$ H $6.799214002 .89660700-3.22062200$ H 4.69686800 4.21111100 -3.38994600 H $2.987002004 .05231600-1.60658800$ H 5.493038001 .311160000 .54598200 H 2.230561003 .274269000 .51007200 H 3.621070000 .729237001 .52294700 H 1.011629002 .208517002 .34329900

\section{1,2-TS2}
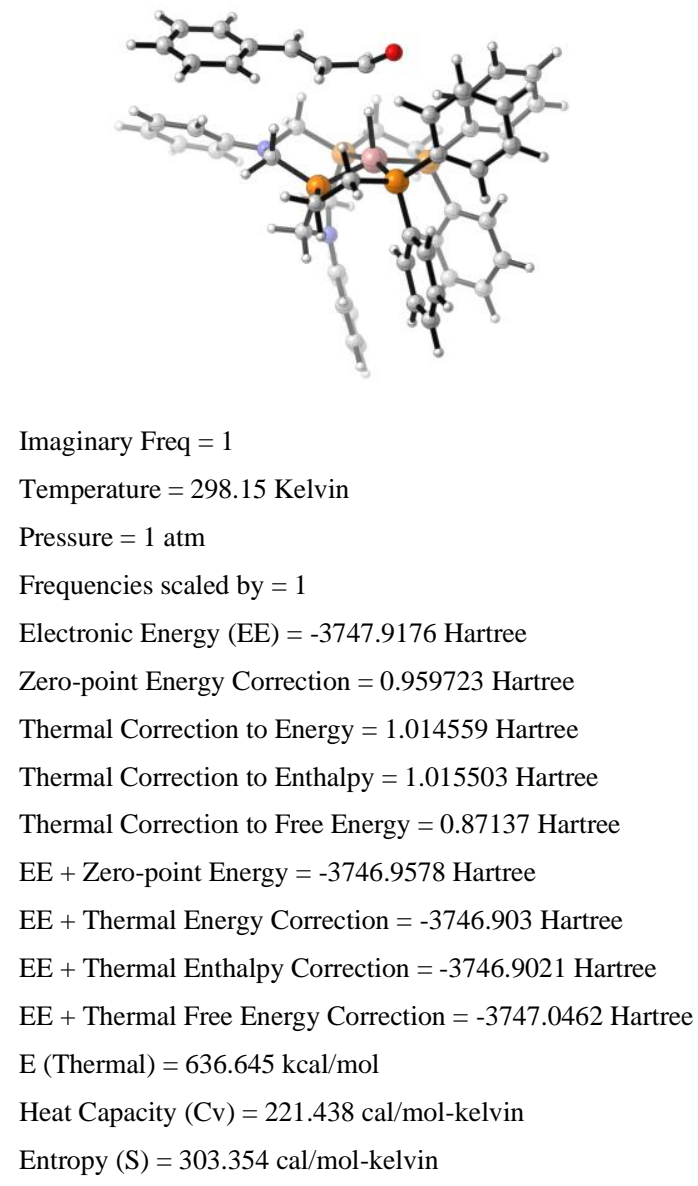
H $6.05935800-3.20531700-0.09013200$ C $5.46302100-1.15658800-0.37788100$ H $6.39064200-0.747591000 .01211400$ C $4.46062700-0.29217800-0.81210900$ H $4.620152000 .78076400-0.76000800$ C $3.25546300-0.79263900-1.31385100$ C $1.18552500-0.41121000-3.30567900$ H $1.592926000 .16248600-4.14292500$ H $1.56520200-1.43300000-3.40211100$ C - $0.34945000-0.41474500-3.32726300$ H -0.75229200 - $1.13712500-4.04693800$ H $-0.733208000 .57835400-3.58745900$ C -2.74091000 -0.51401200-1.57181200 H -2.95331500 $0.56006500-1.66132800$ H -3.18822100 - $1.02540200-2.42787700$ C -5.65326100 -1.01890400 0.38941500 H -5.44395500 -0.20507200 1.07546700 C -6.95103800 - 1.517275000 .30030600 H -7.72018900 -1.08971400 0.93670200 C -7.26687700 -2.52187800-0.60636000 H -8.28105600 -2.90284600 -0.67594500 C $-6.26268300-3.03676600-1.42538500$ H -6.48786700 -3.83160500 -2.13075500 C $-4.96336800-2.55791500-1.32948300$ H -4.18013200 -2.99321800 - 1.94453600 C -4.64124500 - $1.53579200-0.42446200$ C - $2.87890500-0.467420000 .90672000$ H -3.37510400 -1.00351000 1.72266200 H -3.13739100 0.596644000 .98139900 C -0.94428300 -2.52115400 1.05584300 H -0.43800600 -2.86026400 1.95854000 H - $1.96231200-2.941757001 .06151400$ C $1.26867200-4.485086001 .12022400$ H $1.25514900-3.820731001 .97588200$ C 2.07379100 -5.61278500 1.17498900 H 2.63587200 -5.81080700 2.08381000 C $2.18157900-6.474003000 .08412000$ H $2.81731800-7.352195000 .12971600$ C $1.44301200-6.18755400-1.05649900$ H 1.48704100 -6.85116200 -1.91557300 C $0.61853300-5.06573600-1.11688200$ H 0.02906700 -4.91001400 -2.01255000 C $0.52912500-4.17196600-0.03617200$ C $-0.75945400-2.59211400-1.40044800$ H - $1.72951000-3.07598600-1.60663800$ H $-0.05993300-2.90000700-2.18198600$ C - $0.76119900-0.131280002 .90320700$ H - $1.70620700-0.051412003 .45134800$ H $-0.15420400-0.902023003 .39207000$ C 0.005729001 .200438002 .88033500
Н 0.456381001 .421751003 .85333000 H -0.66434400 2.02345800 2.61206000 C 3.177068000 .341107003 .53294900 H 3.070275001 .357878003 .90271300 C $4.02848800-0.540614004 .18803500$ H $4.56865600-0.214319005 .07218000$ C $4.19406500-1.838453003 .70475500$ H $4.86939100-2.523793004 .20918700$ C $3.49611500-2.250263002 .57535100$ H 3.62880700 -3.25240400 2.17948100 C $2.63390300-1.368208001 .92544800$ H $2.08847800-1.683692001 .04007600$ C $2.47693000-0.062283002 .38813900$ C 1.461217003 .852713001 .50714000 H 0.377477003 .845526001 .54000600 C 2.115744005 .069916001 .34323800 H 1.530677005 .982974001 .29937000 C 3.498718005 .107487001 .19781000 H 4.007127006 .057204001 .05835700 C 4.228725003 .921128001 .21365600 H 5.307506003 .941592001 .08923500 C 3.577213002 .704489001 .37997300 H 4.154918001 .784820001 .38902100 C 2.186225002 .659897001 .53564900 H -0.56790600 $1.83388600-0.47510400$ C -6.26619400 2.28892000 2.21025400 C -7.42144500 2.034833001.47117500 C -7.35644300 2.038291000 .08110500 C -6.14870900 2.28958700-0.56087000 C -4.978218002 .545024000 .16488500$ C -5.060994002 .541946001 .56617800$ C -3.71984400 $2.76683500-0.56234000$ C - $2.505466002 .97207500-0.03963500$ C -1.29113800 3.20902500-0.87897100 O -0.56561900 4.19620600 -0.62021900 H -6.30591700 2.29403300 3.29586900 H -8.36251700 1.838982001 .97660300 H -8.24620000 $1.83520100-0.50736600$ H -6.10544500 2.27983300 -1.64707800 H -4.17471700 2.74472800 2.16078500 H -3.80428100 2.73493000 -1.65015100 H -2.34511200 3.070178001 .03252900 H -1.40982100 2.87640800 -1.93129200

\section{1,2-TS3}




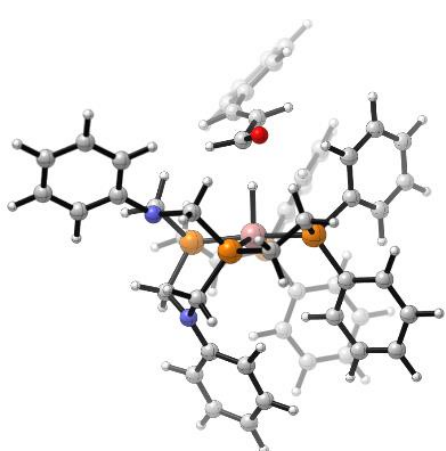

Imaginary Freq $=1$

Temperature $=298.15$ Kelvin

Pressure $=1 \mathrm{~atm}$

Frequencies scaled by $=1$

Electronic Energy $(\mathrm{EE})=-3747.9226$ Hartree

Zero-point Energy Correction $=0.960086$ Hartree

Thermal Correction to Energy $=1.014929$ Hartree

Thermal Correction to Enthalpy $=1.015874$ Hartree

Thermal Correction to Free Energy $=0.87011$ Hartree

$\mathrm{EE}+$ Zero-point Energy = -3746.9625 Hartree

$\mathrm{EE}+$ Thermal Energy Correction $=-3746.9076$ Hartree

$\mathrm{EE}+$ Thermal Enthalpy Correction $=-3746.9067$ Hartree

$\mathrm{EE}+$ Thermal Free Energy Correction $=-3747.0524$ Hartree

$\mathrm{E}($ Thermal $)=636.878 \mathrm{kcal} / \mathrm{mol}$

Heat Capacity $(\mathrm{Cv})=221.197 \mathrm{cal} / \mathrm{mol}-\mathrm{kelvin}$

Entropy $(\mathrm{S})=306.785 \mathrm{cal} / \mathrm{mol}-\mathrm{kelvin}$

Co -0.383202000 .259257000 .16184600$

$\mathrm{N}-1.208761003 .950668000 .14065500$

N -3.52960600 $1.33898500-1.00683100$

P $0.23386200-1.08227500-1.43819400$

P - $0.764168001 .69259900-1.34486100$

P - 1.813084001 .464472001 .18547700

P -0.36620900 -0.99365400 1.94726400

C $2.51780300-2.21777300-2.71497800$

H $1.87189900-2.42380600-3.56518700$

C $3.84636200-2.62083300-2.75915600$

H $4.23619200-3.10111800-3.65231300$

C $4.67431300-2.42052300-1.65346800$

H $5.71467700-2.72972600-1.68824300$

C $4.16663900-1.81489600-0.51190800$

H $4.80077900-1.653372000 .35318100$

C $2.84092300-1.38679000-0.48033700$

H $2.44914600-0.885087000 .39518400$

C $2.00272600-1.58573200-1.57396600$

C -1.89492700 - $2.77517600-2.21110900$

$\mathrm{H}-2.42167700-1.87087700-2.50382400$

C - $2.56425500-3.99141300-2.29977800$

H - $3.57557300-4.01185400-2.69384800$

C $-1.95087800-5.16279100-1.86215500$
H - $2.47788700-6.11079000-1.92015800$

C $-0.66108000-5.10896000-1.34317400$

H - $0.17530300-6.01534600-0.99327800$

C $0.01658000-3.89473500-1.27418000$

H $1.02717400-3.86947000-0.87717000$

C $-0.59032200-2.71149800-1.70867200$

C $-0.04071400-0.22575600-3.08859100$

H $0.63285300-0.61250300-3.85833500$

H -1.06494300 -0.44430600 -3.41103100

C $0.132004001 .28643000-2.90466100$

H -0.23016500 $1.85476700-3.76942900$

H $1.188052001 .53516200-2.74521900$

C $-0.354839003 .45208000-0.94065600$

H $0.718236003 .52212700-0.70743300$

H $-0.537943004 .07152800-1.82474200$

C -0.556671006 .014038001 .34040800$

H -0.06823500 5.430088002 .11434400

C -0.556954007 .405286001 .42083700$

H -0.07444700 7.888868002 .26518700

C - 1.154610008 .171408000 .42624400

H - 1.149397009 .255057000 .49133200

C - $1.766246007 .53490500-0.65292700$

H - $2.248721008 .12113300-1.42969400$

C $-1.783924006 .14838500-0.72936900$

H -2.28921000 $5.65022100-1.55292700$

C - 1.173938005 .371881000 .26327100

C -1.23045500 3.221632001 .41214900

H -1.94792800 3.742961002 .05667000

H -0.26965300 3.172842001 .94362100

C -3.46939400 1.822548000 .35772900

H -4.29319500 1.405138000 .93858900

H -3.59941600 2.91196000 0.33487700

C $-4.58792900-0.76999200-0.35476600$

H $-4.29567600-0.634445000 .67977900$

C $-5.32294400-1.90000700-0.69307200$

H $-5.61493900-2.584517000 .09768700$

C $-5.66191700-2.17019600-2.01446300$

H -6.23379300 - $3.05597800-2.27209100$

C $-5.25434500-1.27341200-2.99870500$

H $-5.51392900-1.45012500-4.03887300$

C $-4.54197300-0.12670800-2.67025600$

H $-4.301945000 .57740000-3.45983000$

C $-4.189759000 .15420600-1.33501700$

C $-2.559166001 .92882500-1.90274600$

H - $2.755564003 .00421300-2.01288800$

$\mathrm{H}-2.640693001 .47223900-2.88876900$

C -2.09917500 0.896510002 .92665700

H -2.31886000 1.729567003 .60303200

H -2.96216200 0.218684002 .92645400

C -0.822913000 .149481003 .33883900$ 
H -0.95716100 -0.38913900 4.28251000 H 0.003862000 .863424003 .44807300 C - $1.58550600-3.083528003 .42581000$ H $-0.91709600-2.803189004 .23628600$ C -2.45559800-4.15293400 3.59542800 H -2.48192200 -4.68441200 4.54243200 C -3.28124500-4.55414700 2.54385600 H -3.94842200 -5.40207600 2.67130400 C -3.24143000 -3.87152200 1.33408800 H -3.86248600 -4.18490600 0.50067800 C -2.38323900 -2.78419000 1.17418600 H - $2.35767800-2.241911000 .23639000$ C - $1.54114700-2.388070002 .20942000$ C $2.19859800-1.038626003 .12080600$ H 2.033435000 .007679003 .36808400 C $3.43505300-1.619589003 .39623800$ H 4.20154200 -1.03013700 3.89214900 C $3.69049300-2.940355003 .03720700$ H $4.65780300-3.387225003 .24847800$ C $2.69889200-3.686166002 .40486000$ H $2.88988700-4.716054002 .11763500$ C $1.46116900-3.112906002 .13427900$ H $0.69497300-3.703306001 .63924400$ C $1.20026100-1.783395002 .48560400$ H 0.963323000 .999803000 .76430600 C $7.206657000 .36942200-0.47204700$ C $7.299627000 .21995300-1.85457500$ C $6.253067000 .65493800-2.66386200$ C $5.121433001 .22542700-2.09315800$ C $5.002723001 .35572800-0.70453100$ C 6.072277000 .936128000 .09706100 C $3.755900001 .87896700-0.13131200$ C 3.235100001 .535858001 .05181800 C 1.949689002 .098273001 .54315400 O 1.666999002 .138245002 .76525700 H 8.026256000 .050129000 .16563100 H $8.18680600-0.22245500-2.29818900$ H $6.316298000 .54517600-3.74258300$ H $4.299714001 .54770500-2.72826800$ H 6.012313001 .062704001 .17447900 H $3.176999002 .54565500-0.77439100$ H 3.735174000 .830377001 .71179100 H 1.566887002 .892787000 .88120400

\section{1,4-TS1}

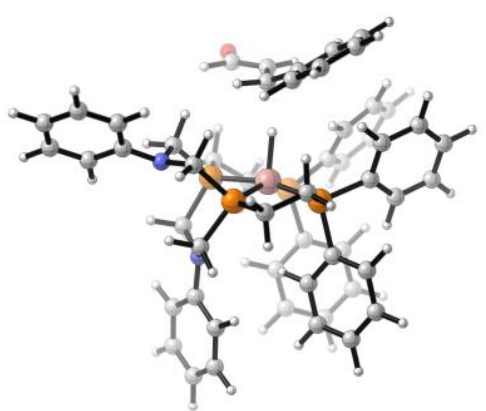

Imaginary Freq = 1

Temperature $=298.15$ Kelvin

Pressure $=1 \mathrm{~atm}$

Frequencies scaled by $=1$

Electronic Energy $(\mathrm{EE})=-3747.9123$ Hartree

Zero-point Energy Correction $=0.959137$ Hartree

Thermal Correction to Energy $=1.014148$ Hartree

Thermal Correction to Enthalpy $=1.015093$ Hartree

Thermal Correction to Free Energy $=0.87042$ Hartree

$\mathrm{EE}+$ Zero-point Energy $=-3746.9532$ Hartree

$\mathrm{EE}+$ Thermal Energy Correction $=-3746.8981$ Hartree

$\mathrm{EE}+$ Thermal Enthalpy Correction $=-3746.8972$ Hartree

$\mathrm{EE}+$ Thermal Free Energy Correction $=-3747.0419$ Hartree

$\mathrm{E}($ Thermal $)=636.388 \mathrm{kcal} / \mathrm{mol}$

Heat Capacity $(\mathrm{Cv})=221.688 \mathrm{cal} / \mathrm{mol}-\mathrm{kelvin}$

Entropy $(\mathrm{S})=304.489 \mathrm{cal} / \mathrm{mol}-\mathrm{kelvin}$

Co $-0.05246200-0.280041000 .03837800$

N $-3.58732900-1.70742800-0.26082200$

$\mathrm{N}-2.742773001 .812523000 .00086000$

P 0.951798000 .632124001 .73295000

P - $1.73667400-0.428575001 .30071500$

P - $1.57088400-0.25805000-1.42277100$

P $1.284960000 .24686200-1.58257600$

C 3.273486000 .486203003 .36878500

H 2.869868001 .334888003 .91625200

C $4.49674000-0.055130003 .74638000$

H 5.034478000 .361144004 .59326300

C $5.03186100-1.130058003 .03663500$

H $5.99057100-1.548794003 .32911400$

C $4.33501700-1.665939001 .95945800$

H $4.74757500-2.496911001 .39513400$

C $3.10542000-1.128405001 .58769200$

H $2.55034900-1.546603000 .75447700$

C $2.57062800-0.041249002 .27822500$

C 0.132518003 .303742001 .69293200

H -0.865384002 .890837001 .80293800$

C 0.264764004 .673593001 .50902000

H -0.62321000 5.300357001 .50940100

C 1.527331005 .223535001 .29199900 
H 1.638745006 .292253001 .13433900 C 2.643134004 .393529001 .27069100 H 3.630502004 .813645001 .10174400 C 2.505652003 .019780001 .46652800 H 3.388337002 .387348001 .45781800 C 1.246438002 .454504001 .67900700 C -0.107439000 .488688003 .28813900$ H 0.518757000 .175463004 .12821700 H -0.49511700 1.484509003 .52397700 C - $1.25903100-0.501952003 .07627500$ H -2.10248900 -0.30593000 3.74809000 H -0.91495800 -1.52282000 3.26326300 C -2.90749000 -1.84123700 1.02795800 H -2.34713200 -2.78449500 1.10272300 H -3.65976300 -1.84408100 1.82136600 C $-4.98317300-3.50399500-1.23182300$ H $-4.15777000-3.84098000-1.85079100$ C -6.20917100 -4.16294300 - 1.30558300 H $-6.32339200-4.99803600-1.99054500$ C -7.27221400 -3.77047300 -0.50120800 H -8.22402200 -4.28907100 -0.55765500 C -7.10443200 -2.70024700 0.37682900 H -7.92991200 -2.37455100 1.00285500 C $-5.89145600-2.028784000 .44132000$ H -5.77471100 -1.17358000 1.10162200 C $-4.81205600-2.42629500-0.35843700$ C - $2.72949600-1.72667900-1.43487100$ H -3.37174300 - $1.66519000-2.31987200$ H -2.11434700 -2.63914400 -1.51406200 C -2.86427100 1.10286400-1.26632900 H -2.70477400 $1.79894200-2.08854000$ H -3.87263800 0.67466000 - 1.37699300 C -2.67494800 4.04741500-0.98963000 H -2.17685100 $3.66072000-1.87069400$ C -2.88112400 5.41606900-0.89811900 H -2.57034600 $6.04789300-1.72592100$ C -3.45859600 5.983137000 .23649400 H -3.61237000 7.055061000 .30542800 C -3.84648500 5.139651001.26954900 H -4.32067300 5.54734500 2.15789400 C -3.65815500 3.762062001 .18225500 H -4.01626000 3.142811001 .99596400 C -3.04399900 3.182922000 .05817000 C - 2.935473001 .011792001 .19408400 $\mathrm{H}-3.975418000 .658878001 .30524400$ H -2.69811600 1.626374002 .06603900 C -0.90812000 -0.19357200 -3.16572400 H - $1.46751900-0.85206200-3.83910700$ H - $1.023081000 .83323700-3.53199400$ C $0.58232800-0.56305900-3.10706200$
H $1.10946100-0.24713800-4.01334600$ H $0.70447400-1.64442500-2.99168800$ C $2.205508002 .37113000-3.22700200$ H $2.863966001 .63590000-3.68285500$ C $2.234750003 .68624900-3.67361500$ H $2.898542003 .96685000-4.48640800$ C $1.422490004 .64592200-3.06880000$ H 1.457489005 .67790800 -3.40644900 C $0.573283004 .27891300-2.03191800$ H -0.05412700 5.01811100 -1.54376300 C $0.531884002 .95436900-1.59695700$ H -0.13216800 2.65953500 -0.78929200 C $1.356769001 .99357100-2.17740600$ C $3.53089000-1.42918000-1.96813000$ H $2.84333500-2.17518800-2.35225900$ C $4.88053100-1.75232300-1.84633100$ H $5.21955600-2.73614100-2.15552500$ C $5.78078500-0.82876000-1.32580600$ H $6.83097500-1.08674500-1.22478700$ C $5.328270000 .42893800-0.93346000$ H $6.022299001 .15581900-0.52195100$ C $3.984680000 .75597500-1.06448600$ H $3.642069001 .74242500-0.76645300$ C $3.07049700-0.17020400-1.58219500$ H $0.34929200-1.861948000 .22817400$ C $3.45822200-4.82878000-1.24581800$ C $2.92389100-4.91555500-2.52743800$ C $1.61111000-4.49608400-2.74905900$ C $0.85580900-3.99555500-1.69641400$ C $1.38031500-3.90372700-0.40076200$ C $2.69460200-4.32984000-0.19305900$ C $0.49529400-3.487781000 .72021200$ C $0.83853400-3.628822002 .05716700$ C -0.17086600 -3.80371400 3.04440700 O -0.01778800 -3.876399004.26711200 H $4.47451100-5.16305700-1.05663400$ H $3.51720800-5.31420300-3.34492000$ H $1.17610100-4.56737000-3.74222600$ H -0.16728200 -3.66936900 -1.86940000 H $3.11439800-4.304036000 .80681400$ H $-0.55739600-3.643506000 .49034000$ H $1.86569500-3.561917002 .40221800$ H -1.20670400 -3.899662002.63466600

\section{1,4-TS2}




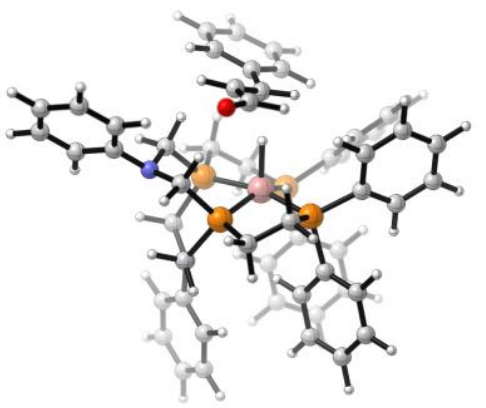

Imaginary Freq = 1

Temperature $=298.15$ Kelvin

Pressure $=1 \mathrm{~atm}$

Frequencies scaled by $=1$

Electronic Energy $(\mathrm{EE})=-3747.9181$ Hartree

Zero-point Energy Correction $=0.959466$ Hartree

Thermal Correction to Energy $=1.01451$ Hartree

Thermal Correction to Enthalpy $=1.015454$ Hartree

Thermal Correction to Free Energy $=0.87051$ Hartree

$\mathrm{EE}+$ Zero-point Energy $=-3746.9586$ Hartree

$\mathrm{EE}+$ Thermal Energy Correction $=-3746.9036$ Hartree

$\mathrm{EE}+$ Thermal Enthalpy Correction $=-3746.9026$ Hartree

$\mathrm{EE}+$ Thermal Free Energy Correction $=-3747.0476$ Hartree

$\mathrm{E}($ Thermal $)=636.615 \mathrm{kcal} / \mathrm{mol}$

Heat Capacity $(\mathrm{Cv})=221.539 \mathrm{cal} / \mathrm{mol}-\mathrm{kelvin}$

Entropy $(S)=305.061 \mathrm{cal} / \mathrm{mol}-\mathrm{kel}$ in

Co $0.07290500-0.218123000 .16026000$

N 3.613180001 .224143000 .30432800

N $0.507970003 .13826900-0.41871200$

$\mathrm{P}-1.26948200-0.52968200-1.52416300$

P $1.402945000 .64307900-1.23627600$

P 1.095984001 .166694001 .41891800

P - $1.17138500-0.588023001 .89608400$

C $-2.81674000-2.31635700-3.09728600$

H -2.92039600 -1.49171400 -3.79848400

C $-3.42254700-3.53408900-3.38203400$

H $-3.97559800-3.65965800-4.30843000$

C $-3.32555100-4.59060100-2.47555100$

H -3.80001300 -5.54225700 -2.69720400

C $-2.62498600-4.41863200-1.28752500$

H $-2.54871000-5.22876200-0.56878000$

C $-2.01344000-3.19643500-1.01006500$

H - $1.45331300-3.06765300-0.09077000$

C -2.09496400 -2.13630400-1.90942000

C -2.52288900 1.95103400-1.98101600

H - $1.532795002 .31095200-2.24070500$

C -3.56667000 2.86726200-1.93012600

H -3.37335800 $3.90956400-2.16817800$

C $-4.839407002 .44672100-1.54922300$
H -5.65817000 $3.15864100-1.49975100$

C -5.05263200 $1.11097400-1.22233100$

H -6.03983900 $0.77625500-0.91698200$

C -4.00341900 $0.19666300-1.27674000$

H -4.18598300 -0.84175100 -1.01455500

C $-2.722520000 .60407000-1.66154500$

C $-0.35362800-0.19269100-3.14587700$

H -0.56230700 -0.99953000 -3.85365800

H - $0.755320000 .72891300-3.57895800$

C $1.15797300-0.06481800-2.91591500$

H $1.643914000 .52995900-3.69809200$

H 1.62982200 -1.05224700 -2.89771800

C $3.205413000 .46495500-0.88088600$

H $3.40282200-0.60848500-0.77565900$

H $3.779665000 .83183100-1.73758900$

C 5.746684000 .525373001 .36334600

H $5.22999300-0.152448002 .03824500$

C 7.135467000 .626271001 .43054800

H 7.672387000 .030128002 .16239900

C 7.829683001 .467163000 .56750900

H 8.911691001 .538000000 .62407800

C $7.124386002 .22434800-0.36687400$

H $7.653668002 .89541200-1.03736100$

C $5.739286002 .14156200-0.42960600$

H $5.180311002 .75115600-1.13521300$

C 5.038687001 .287186000 .42862500

C 2.923512000 .881818001 .54599800

H 3.333560001 .522168002 .33448100

H $3.05477100-0.164065001 .85036100$

C 1.079611002 .972609000 .90943300

H 0.488250003 .528017001 .63646700

H 2.108323003 .364777000 .93974100

C - 1.228227004 .716489000 .24345700

H - 1.334545004 .212299001 .19737000

C -2.07430700 $5.77588600-0.04711200$

H -2.79295200 6.094255000 .70334800

C -2.02709600 6.41446600 - 1.28585000

H -2.69513800 7.23942100 -1.51103600

C -1.09725900 $5.97651600-2.22054200$

H - $1.023735006 .46691200-3.18723800$

C $-0.229242004 .92494800-1.93258500$

H $0.510215004 .64544000-2.67438400$

C -0.28661800 4.25864100 -0.69760600

C $1.247906002 .50186000-1.48920200$

H 2.246192002 .94818400 -1.63947400

H $0.685602002 .61566300-2.41962500$

C 0.553847001 .087295003 .19500300

H 1.390791001 .244150003 .88270100

H -0.182655001 .881770003 .36224400$

C -0.09842700 -0.28528300 3.39120700 
H -0.67790800 -0.33163600 4.31896500 H $0.67253300-1.062346003 .42429700$ C -3.37848800 0.41850300 3.38999300 H -3.27747800 -0.46328800 4.01780600 C -4.352726001 .363448003 .69033900$ H -4.99837500 1.218702004 .55177600 C -4.500983002.493086002.88569900 H -5.26797200 3.226990003 .11648200 C -3.664977002.676715001.79014400 H -3.77448000 3.545975001 .14876500 C -2.684819001.731120001.49334200 $\mathrm{H}-2.027174001 .871633000 .63978200$ C - 2.540250000 .587962002 .28010400 C - $1.12113400-3.287539002 .63435400$ H -0.06640500 -3.12256200 2.83448900 C - $1.64314000-4.571745002 .75691900$ H -0.99676900 -5.38765400 3.06651300 C -2.98931300-4.80666600 2.48528000 H -3.39811600 -5.80829900 2.58096800 C -3.80715500 -3.75170100 2.08972200 $\mathrm{H}-4.85522100$-3.92834000 1.86708400

C -3.28363600 -2.46841300 1.96488000
H -3.92831900 -1.65649600 1.64094600 C $-1.93512500-2.220107002 .24046500$ H $1.04444500-1.545351000 .25304400$ C $3.57999300-3.17131700-3.10211000$ C $2.47783900-3.63502200-3.81429000$ C $1.28309900-3.88052400-3.13673100$ C $1.19871100-3.63752500-1.77304700$ C $2.28320300-3.13294200-1.04793900$ C $3.48740600-2.93124600-1.73184800$ C $2.12235000-2.857754000 .40749700$ C $3.19653300-2.544072001 .23252600$ C $3.07136700-2.569683002 .64546600$ O $3.87701800-2.112313003 .46599400$ H $4.52486700-3.00156500-3.61047100$ H $2.55272800-3.81880200-4.88207000$ H $0.41321300-4.25909500-3.66635000$ H $0.26681400-3.82373600-1.25591100$ H $4.37097100-2.59775800-1.19719200$ H $1.29763100-3.400701000 .86624300$ H $4.11732600-2.123112000 .83713100$ H $2.13313900-3.038790003 .02753500$ 


\section{Characterization data of the hydrogenation products}

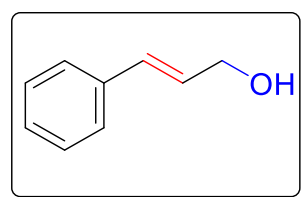

\section{2aa: (E)-3-phenylprop-2-en-1-ol ${ }^{[13]}$}

99\% yield; pale yellow oil; ${ }^{1} \mathrm{H}$ NMR (400 MHz, Chloroform- $d$ ) $\delta 7.35(\mathrm{~m}, 6 \mathrm{H}), 6.64(\mathrm{~d}, J=15.9$ $\mathrm{Hz}, 1 \mathrm{H}), 6.39$ (dt, $J=15.9,5.7 \mathrm{~Hz}, 1 \mathrm{H}), 4.34(\mathrm{~d}, J=5.7 \mathrm{~Hz}, 2 \mathrm{H}), 2.17$ (brs, $1 \mathrm{H}) ;{ }^{13} \mathrm{C}$ NMR $(101$ MHz, Chloroform- $d$ ) $\delta 136.7,131.1,128.6,128.6,127.7,126.5,63.7$;

The obtained spectroscopic data were in agreement with the reported data for compound $\mathbf{2 a a}$;

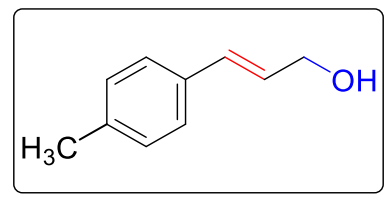

2ab: (E)-3-(p-tolyl)prop-2-en-1-ol ${ }^{[14]}$

95\% yield; colorless oil; ${ }^{1} \mathrm{H}$ NMR (400 MHz, Chloroform- $d$ ) $\delta$ 7.35-7.25 (m, 2H), $7.12(\mathrm{~d}$, $J=7.9 \mathrm{~Hz}, 2 \mathrm{H}), 6.58(\mathrm{dt}, J=15.9,1.5 \mathrm{~Hz}, 1 \mathrm{H}), 6.31(\mathrm{dt}, J=15.9,5.8 \mathrm{~Hz}, 1 \mathrm{H}), 4.40-4.23$ $(\mathrm{m}, 2 \mathrm{H}), 2.34(\mathrm{~s}, 3 \mathrm{H}), 1.60(\mathrm{~d}, J=6.6 \mathrm{~Hz}, 1 \mathrm{H}) ;{ }^{13} \mathrm{C}$ NMR $(101 \mathrm{MHz}$, Chloroform- $d) \delta$

137.6, 133.9, 131.3, 129.4, 127.5, 126.4, 63.9, 21.2;

The obtained spectroscopic data were in agreement with the reported data for compound $\mathbf{2 a b}$;

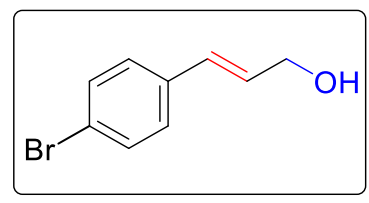

2ac: (E)-3-(4-bromophenyl)prop-2-en-1-ol ${ }^{[15]}$

94\% yield; white solid; ${ }^{1} \mathrm{H}$ NMR (400 MHz, Chloroform- $d$ ) $\delta$ 7.50-7.40 (m, 2H), 7.25 (dd, $J$ $=9.2,2.8 \mathrm{~Hz}, 2 \mathrm{H}), 6.65-6.49(\mathrm{~m}, 1 \mathrm{H}), 6.35(\mathrm{dt}, J=15.9,5.6 \mathrm{~Hz}, 1 \mathrm{H}), 4.39-4.24(\mathrm{~m}, 2 \mathrm{H})$, $1.64(\mathrm{~s}, 2 \mathrm{H}) ;{ }^{13} \mathrm{C}$ NMR $(101 \mathrm{MHz}$, Chloroform- $d$ ) $\delta 135.7,131.7,129.8,129.3,128.0,121.5$,

63.5 ;

The obtained spectroscopic data were in agreement with the reported data for compound 2ac;

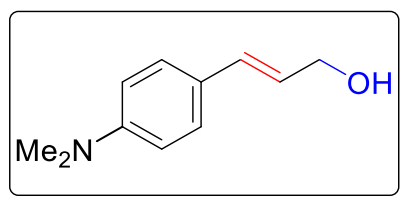

2ad: (E)-3-(4-(dimethylamino)phenyl)prop-2-en-1-ol ${ }^{[16]}$

98\% yield; bright yellow solid; ${ }^{1} \mathrm{H}$ NMR (400 MHz, Chloroform- $d$ ) $\delta 7.28(\mathrm{~d}, J=8.7 \mathrm{~Hz}$, $2 \mathrm{H}), 6.72-6.64(\mathrm{~m}, 2 \mathrm{H}), 6.51(\mathrm{dd}, J=15.8,1.7 \mathrm{~Hz}, 1 \mathrm{H}), 6.17(\mathrm{dt}, J=15.8,6.2 \mathrm{~Hz}, 1 \mathrm{H})$, $4.27(\mathrm{t} J=4.9 \mathrm{~Hz}, 2 \mathrm{H}), 2.96(\mathrm{~s}, 6 \mathrm{H}), 1.51(\mathrm{~s}, 1 \mathrm{H}) ;{ }^{13} \mathrm{C}$ NMR $(101 \mathrm{MHz}$, Chloroform- $d) \delta 150.2,131.9,127.5,125.0$, 124.0, 112.4, 64.3, 40.5;

The obtained spectroscopic data were in agreement with the reported data for compound 2ad;

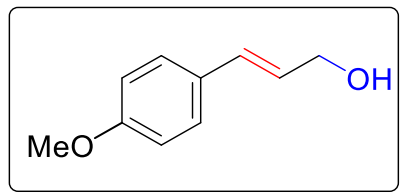

2ae: $(E)-3-(4-m e t h o x y p h e n y l) p r o p-2-e n-1-o I^{[17]}$

97\% yield; colorless oil; ${ }^{1} \mathrm{H}$ NMR (400 MHz, Chloroform- $d$ ) $\delta 7.35-7.30(\mathrm{~m}, 2 \mathrm{H}), 6.91-$ $6.72(\mathrm{~m}, 2 \mathrm{H}), 6.56(\mathrm{dt}, J=15.8,1.6 \mathrm{~Hz}, 1 \mathrm{H}), 6.24(\mathrm{dt}, J=15.8,5.9 \mathrm{~Hz}, 1 \mathrm{H}), 4.30(\mathrm{~d}, J=$ $5.9 \mathrm{~Hz}, 2 \mathrm{H}), 3.81(\mathrm{~s}, 3 \mathrm{H}), 1.49(\mathrm{~s}, 1 \mathrm{H}) ;{ }^{13} \mathrm{C}$ NMR (101 MHz, Chloroform-d) $\delta$ 159.32,

$131.00,129.41,127.69,126.24,114.02,63.97,55.32$.

The obtained spectroscopic data were in agreement with the reported data for compound $2 \mathbf{a e}$; 


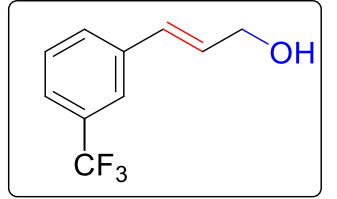

2af: (E)-3-(3-(trifluoromethyl)phenyl)prop-2-en-1-ol ${ }^{[18]}$

91\% yield; white solid; ${ }^{1} \mathrm{H}$ NMR (600 MHz, Chloroform- $d$ ) $\delta 7.62$ (s, 1H), 7.54 (d, $J=7.8 \mathrm{~Hz}$, $1 \mathrm{H}), 7.49(\mathrm{~d}, J=7.9 \mathrm{~Hz}, 1 \mathrm{H}), 7.43(\mathrm{t}, J=7.8 \mathrm{~Hz}, 1 \mathrm{H}), 6.66(\mathrm{dd}, J=16.0,1.7 \mathrm{~Hz}, 1 \mathrm{H}), 6.44$ (dtd, $J=16.0,5.5,1.3 \mathrm{~Hz}, 1 \mathrm{H}), 4.36(\mathrm{dd}, J=5.4,1.5 \mathrm{~Hz}, 2 \mathrm{H}), 1.75$ (brs, $1 \mathrm{H}) ;{ }^{19} \mathrm{~F}$ NMR $(565 \mathrm{MHz}$,

Chloroform- $d$ ) $\delta-62.8 ;{ }^{13} \mathrm{C}$ NMR (151 MHz, Chloroform- $d$ ) $\delta 137.5,130.6,129.6,129.4,129.1,124.3,124.2,124.1$, 123.1, 123.0, 63.3;

The obtained spectroscopic data were in agreement with the reported data for compound 2af;

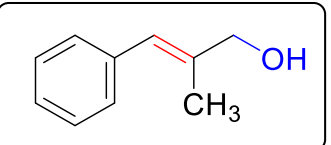

2ag: (E)-2-methyl-3-phenylprop-2-en-1-ol ${ }^{[19]}$

95\% yield; pale yellow oil; ${ }^{1} \mathrm{H}$ NMR (400 MHz, Chloroform-d) $\delta 7.58-7.07(\mathrm{~m}, 5 \mathrm{H}), 6.56$ (s, $1 \mathrm{H}), 4.31-4.12(\mathrm{~m}, 2 \mathrm{H}), 1.93(\mathrm{~s}, 3 \mathrm{H}) ;{ }^{13} \mathrm{C} \mathrm{NMR}\left(101 \mathrm{MHz}, \mathrm{CDCl}_{3}\right) \delta 137.7,137.5,128.9$, 128.2, 126.5, 125.0, 77.4, 77.1, 76.7, 69.0, 15.3;

The obtained spectroscopic data were in agreement with the reported data for compound $\mathbf{2}$ af;

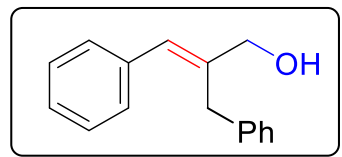

126.3, 66.5, 34.4;

\section{2ah: (E)-2-benzyl-3-phenylprop-2-en-1-ol ${ }^{[20]}$}

95\% yield; colorless oil; ${ }^{1} \mathrm{H}$ NMR $(400 \mathrm{MHz},) \delta 7.36(\mathrm{~d}, J=5.0 \mathrm{~Hz}, 4 \mathrm{H}), 7.35-7.27(\mathrm{~m}, 4 \mathrm{H})$, $7.26(\mathrm{dd}, J=7.3,5.1 \mathrm{~Hz}, 3 \mathrm{H}), 6.84(\mathrm{~s}, 1 \mathrm{H}), 4.18(\mathrm{~s}, 2 \mathrm{H}), 3.75(\mathrm{~s}, 2 \mathrm{H}), 1.75(\mathrm{~s}, 2 \mathrm{H}) ;{ }^{13} \mathrm{C} \mathrm{NMR}$ (101 MHz, Chloroform-d) $\delta 139.7,137.17,128.7,128.6,128.6,128.5,128.4,127.3,126.9$,

The obtained spectroscopic data were in agreement with the reported data for compound $\mathbf{2 a h}$;

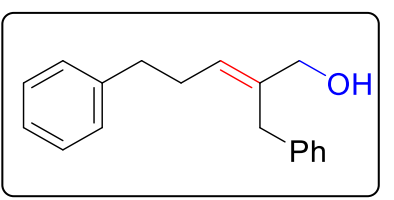

\section{2ai: $(E)$-2-benzyl-5-phenylpent-2-en-1-ol ${ }^{[21]}$}

95\% yield; colorless viscous liquid; ${ }^{1} \mathrm{H}$ NMR (400 MHz, Chloroform- $d$ ) $\delta 7.33-7.21$ (m, $5 \mathrm{H}), 7.19(\mathrm{dd}, J=8.9,2.5 \mathrm{~Hz}, 3 \mathrm{H}), 7.13-7.06(\mathrm{~m}, 2 \mathrm{H}), 5.66(\mathrm{t}, J=7.2 \mathrm{~Hz}, 1 \mathrm{H}), 3.95(\mathrm{~s}, 2 \mathrm{H})$, $3.43(\mathrm{~s}, 2 \mathrm{H}), 2.73(\mathrm{dd}, J=8.8,6.7 \mathrm{~Hz}, 2 \mathrm{H}), 2.51(\mathrm{q}, J=7.5 \mathrm{~Hz}, 2 \mathrm{H}), 1.24(\mathrm{~d}, J=8.8 \mathrm{~Hz}$, 2H); ${ }^{13} \mathrm{C}$ NMR (101 MHz, Chloroform-d) $\delta$ 141.8, 138.2, 128.5, 128.5, 128.4, 127.2 , 126.1, 126.0, 66.7, 35.9, 33.8, 29.9; The obtained spectroscopic data were in agreement with the reported data for compound 2ai;

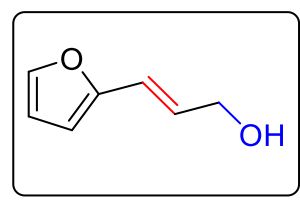

2aj: (E)-3-(furan-2-yl)prop-2-en-1-ol ${ }^{[22]}$

97\% yield; white solid; ${ }^{1} \mathrm{H}$ NMR (600 MHz, Chloroform- $d$ ) $\delta 7.37$ (s, 1H), 6.44 (d, J=15.9 Hz, $1 \mathrm{H}), 6.41-6.19(\mathrm{~m}, 3 \mathrm{H}), 4.29$ (d, $J=5.6 \mathrm{~Hz}, 2 \mathrm{H}), 1.76(\mathrm{~s}, 2 \mathrm{H}) ;{ }^{13} \mathrm{C}$ NMR $(151 \mathrm{MHz}$,

Chloroform- $d$ ) $\delta 152.4,142.0,127.2,119.3,111.3,108.0,63.3$;

The obtained spectroscopic data were in agreement with the reported data for compound 2aj;

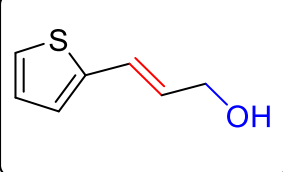




\section{2ak: (E)-3-(thiophen-2-yl)prop-2-en-1-o[ ${ }^{[14]}$}

95\% yield; pale yellow solid; ${ }^{1} \mathrm{H}$ NMR $(600 \mathrm{MHz}$, Chloroform- $d$ ) $\delta 7.18-7.13(\mathrm{~m}, 1 \mathrm{H}), 6.96(\mathrm{~d}, J=1.6 \mathrm{~Hz}, 1 \mathrm{H}), 6.74$ $(\mathrm{dt}, J=15.8,1.6 \mathrm{~Hz}, 1 \mathrm{H}), 6.20(\mathrm{dt}, J=15.7,5.8 \mathrm{~Hz}, 1 \mathrm{H}), 4.27(\mathrm{~d}, J=1.6 \mathrm{~Hz}, 1 \mathrm{H}) ;{ }^{13} \mathrm{C}$ NMR $(151 \mathrm{MHz}, \mathrm{Chloroform}-d) \delta$ $141.79,128.15,127.38,125.82,124.40,124.36,63.38$.

The obtained spectroscopic data were in agreement with the reported data for compound 2ak;

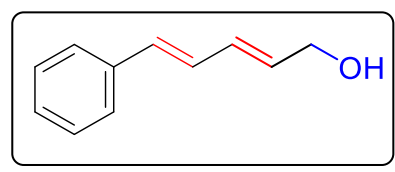

2al: $(2 E, 4 E)-5$-phenylpenta-2,4-dien-1-ol ${ }^{[14]}$

98\% yield; colorless oil; ${ }^{1} \mathrm{H}$ NMR (600 MHz, Chloroform- $d$ ) $\delta 7.38$ (d, $\left.J=7.7 \mathrm{~Hz}, 2 \mathrm{H}\right)$, $7.30(\mathrm{t}, J=7.6 \mathrm{~Hz}, 2 \mathrm{H}), 7.22(\mathrm{t}, J=7.4 \mathrm{~Hz}, 1 \mathrm{H}), 6.78(\mathrm{dd}, J=15.6,10.5 \mathrm{~Hz}, 1 \mathrm{H}), 6.54(\mathrm{~d}$, $J=15.6 \mathrm{~Hz}, 1 \mathrm{H}), 6.41(\mathrm{dd}, J=15.2,10.5 \mathrm{~Hz}, 1 \mathrm{H}), 5.95(\mathrm{dt}, J=15.1,5.9 \mathrm{~Hz}, 1 \mathrm{H}), 4.23(\mathrm{~d}, J=5.9 \mathrm{~Hz}, 2 \mathrm{H}), 1.75(\mathrm{~s}, 1 \mathrm{H})$; ${ }^{13} \mathrm{C}$ NMR (151 MHz, Chloroform-d) $\delta$ 137.2, 132.8, 132.6, 131.6, 128.7, 128.2, 127.7, 126.4, 63.4;

The obtained spectroscopic data were in agreement with the reported data for compound $\mathbf{2 a l}$;

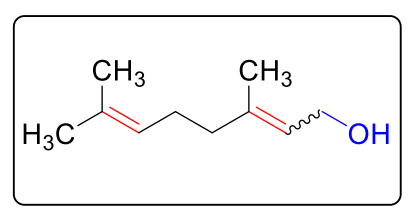

\section{2am: 3,7-dimethylocta-2,6-dien-1-ol ${ }^{[23]}$}

97\% yield; pale yellow oil; ${ }^{1} \mathrm{H}$ NMR (400 MHz, Chloroform- $d$ ) $\delta 5.52-5.33(\mathrm{~m}, 1 \mathrm{H})$, 5.19-5.01 (m, 1H), $4.14(\mathrm{~d}, J=6.9 \mathrm{~Hz}, 2 \mathrm{H}), 4.08(\mathrm{~d}, J=7.2 \mathrm{~Hz}, 1 \mathrm{H}), 2.15-2.06(\mathrm{~m}, 3 \mathrm{H})$, $2.03(\mathrm{~d}, J=8.3 \mathrm{~Hz}, 2 \mathrm{H}), 1.74(\mathrm{~s}, 1 \mathrm{H}), 1.68(\mathrm{~d}, J=3.1 \mathrm{~Hz}, 7 \mathrm{H}), 1.60(\mathrm{~s}, 4 \mathrm{H}), 1.25$ (s, 3H); ${ }^{13} \mathrm{C}$ NMR $\left(101 \mathrm{MHz}, \mathrm{CDCl}_{3}\right) \delta 139.9,139.7,132.4,131.7,124.4,123.9,123.8,123.4$, $77.3,59.4,59.0,39.5,29.7,26.5,26.4,25.7,23.4,17.7,17.6,16.3$;

The obtained spectroscopic data were in agreement with the reported data for compound 2am;

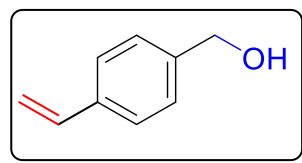

\section{2an: (4-vinylphenyl)methanol ${ }^{[24]}$}

95\% yield; colorless oil; ${ }^{1} \mathrm{H}$ NMR (400 MHz, Chloroform- $d$ ) $\delta 7.44-7.37$ (m, 2H), $7.35-7.25$ $(\mathrm{m}, 2 \mathrm{H}), 6.71(\mathrm{dd}, J=17.6,10.9 \mathrm{~Hz}, 1 \mathrm{H}), 5.75(\mathrm{dd}, J=17.6,0.9 \mathrm{~Hz}, 1 \mathrm{H}), 5.24(\mathrm{dd}, J=10.9,0.9$ $\mathrm{Hz}, 1 \mathrm{H}), 4.67$ (s, 2H), 1.81 (s, 1H); ${ }^{13} \mathrm{C}$ NMR (101 MHz, Chloroform-d) $\delta 140.44,137.05$,

$136.48,127.22,126.42,113.92,65.12$;

The obtained spectroscopic data were in agreement with the reported data for compound 2an;

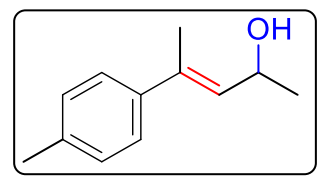

2ao: (E)-4-(p-tolyl)pent-3-en-2-ol ${ }^{[25]}$

96 yield; yellow oil; ${ }^{1} \mathrm{H}$ NMR (600 MHz, Chloroform- $d$ ) $\delta 7.35-7.25(\mathrm{~m}, 2 \mathrm{H}), 7.13(\mathrm{~d}, J=7.9$ $\mathrm{Hz}, 2 \mathrm{H}), 5.77(\mathrm{dq}, J=8.4,1.4 \mathrm{~Hz}, 1 \mathrm{H}), 4.83-4.68(\mathrm{~m}, 1 \mathrm{H}), 2.34(\mathrm{~s}, 3 \mathrm{H}), 2.07$ (d, $J=1.4 \mathrm{~Hz}$ 3H), $1.33(\mathrm{~d}, J=6.3 \mathrm{~Hz}, 3 \mathrm{H}) ;{ }^{13} \mathrm{C}$ NMR $(151 \mathrm{MHz}$, Chloroform- $d) \delta$ 140.0, 137.0, 136.1, 131.2, 129.0, 125.7, 65.3, 23.6, 21.1, 16.1;

The obtained spectroscopic data were in agreement with the reported data for compound 2ao;

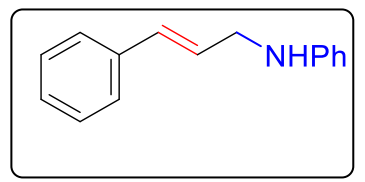

\section{2ap: $N$-cinnamylaniline ${ }^{[26]}$}

97\% yield; yellow solid; ${ }^{1} \mathrm{H}$ NMR (400 MHz, Chloroform- $d$ ) $\delta 7.43-7.32(\mathrm{~m}, 2 \mathrm{H}), 7.29$ (dd, $J=8.4,6.7 \mathrm{~Hz}, 2 \mathrm{H}), 7.25-7.11(\mathrm{~m}, 3 \mathrm{H}), 6.71(\mathrm{dd}, J=8.0,6.7 \mathrm{~Hz}, 1 \mathrm{H}), 6.72-6.61(\mathrm{~m}, 2 \mathrm{H})$, 
$6.64-6.52(\mathrm{~m}, 1 \mathrm{H}), 6.30(\mathrm{dt}, J=15.9,5.7 \mathrm{~Hz}, 1 \mathrm{H}), 3.90(\mathrm{dd}, J=5.9,1.7 \mathrm{~Hz}, 2 \mathrm{H}), 3.79(\mathrm{~s}, 1 \mathrm{H}) ;{ }^{13} \mathrm{C} \mathrm{NMR}(101 \mathrm{MHz}$, Chloroform- $d$ ) $\delta 147.0,135.8,130.5,128.3,127.5,127.4,126.5,126.0,125.3,116.6,112.0,111.7,45.2$;

The obtained spectroscopic data were in agreement with the reported data for compound 2ap;

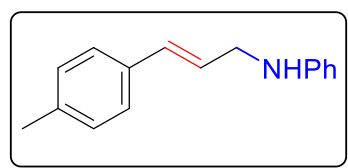

\section{2aq: (E)-N-(3-(p-tolyl)allyl)aniline $\mathrm{e}^{[27]}$}

98\% yield; white solid; ${ }^{1} \mathrm{H}$ NMR (400 MHz, Chloroform-d) $\delta 7.28-7.22$ (m, 2H), $7.22-7.13$ $(\mathrm{m}, 2 \mathrm{H}), 7.11(\mathrm{~s}, 1 \mathrm{H}), 6.71(\mathrm{tt}, \mathrm{J}=7.4,1.1 \mathrm{~Hz}, 1 \mathrm{H}), 6.70-6.61(\mathrm{~m}, 2 \mathrm{H}), 6.57(\mathrm{dt}, J=15.7,1.6$ $\mathrm{Hz}, 1 \mathrm{H}), 6.26(\mathrm{dt}, J=15.8,5.8 \mathrm{~Hz}, 1 \mathrm{H}), 3.89(\mathrm{dd}, J=5.9,1.6 \mathrm{~Hz}, 2 \mathrm{H}), 3.79(\mathrm{~s}, 1 \mathrm{H}), 2.32$ (s, $3 \mathrm{H}) ;{ }^{13} \mathrm{C}$ NMR (101 MHz, Chloroform-d) $\delta$ 148.2, 137.4, 134.1, 131.5, 129.4, 129.3, 126.3, 126.0, 117.6, 113.1, 46.3, 21.3;

The obtained spectroscopic data were in agreement with the reported data for compound 2aq;

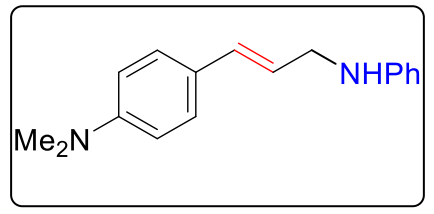

\section{2ar: (E)-N,N-dimethyl-4-(3-(phenylamino)prop-1-en-1-yl)aniline ${ }^{[28]}$}

99\% yield; yellow solid; ${ }^{1} \mathrm{H}$ NMR (400 MHz, Chloroform-d) $\delta 7.27(\mathrm{dd}, \mathrm{J}=9.2,2.4 \mathrm{~Hz}$, $2 \mathrm{H}), 7.23-7.16(\mathrm{~m}, 2 \mathrm{H}), 6.79-6.65(\mathrm{~m}, 5 \mathrm{H}), 6.55(\mathrm{~d}, \mathrm{~J}=15.8 \mathrm{~Hz}, 1 \mathrm{H}), 6.14(\mathrm{dt}, \mathrm{J}=$ 15.6, 6.0 Hz, 1H), $3.89(\mathrm{dd}, \mathrm{J}=6.0,1.5 \mathrm{~Hz}, 2 \mathrm{H}), 3.70(\mathrm{brs}, 1 \mathrm{H}), 2.94(\mathrm{~s}, 6 \mathrm{H}) ;{ }^{13} \mathrm{C} \mathrm{NMR}$ (101 MHz, Chloroform-d) $\delta 150.1,148.3,131.8,129.3,127.3,125.4,122.4,117.5,113.1,112.5,46.6,40.6$;

The obtained spectroscopic data were in agreement with the reported data for compound $2 \mathbf{a r}$;

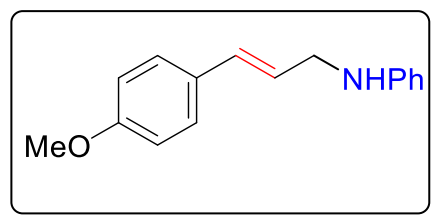

\section{2as: (E)- $N$-(3-(4-methoxyphenyl)allyl)aniline ${ }^{[26]}$}

$98 \%$ yield; white solid; ${ }^{1} \mathrm{H}$ NMR (600 MHz, Chloroform- $d$ ) $\delta 7.35-7.27$ (m, 2H), 7.18 $(\mathrm{tt}, J=7.3,1.4 \mathrm{~Hz}, 2 \mathrm{H}), 6.87-6.81(\mathrm{~m}, 2 \mathrm{H}), 6.72(\mathrm{tq}, J=7.2,1.2 \mathrm{~Hz}, 1 \mathrm{H}), 6.68-6.63$ $(\mathrm{m}, 2 \mathrm{H}), 6.55(\mathrm{dd}, J=16.0,1.6 \mathrm{~Hz}, 1 \mathrm{H}), 6.18(\mathrm{dtd}, J=15.8,5.9,1.4 \mathrm{~Hz}, 1 \mathrm{H}), 3.89$ (dt, $J=5.9,1.5 \mathrm{~Hz}, 2 \mathrm{H}), 3.85(\mathrm{~s}, 1 \mathrm{H}), 3.79$ (d, $J=1.4 \mathrm{~Hz}, 4 \mathrm{H}) .{ }^{13} \mathrm{C}$ NMR $(151 \mathrm{MHz}$, Chloroform- $d$ ) $\delta 159.2,148.2,131.1$, 129.7, 129.3, 127.5, 124.8, 117.6, 114.0, 113.1, 55.3, 46.3;

The obtained spectroscopic data were in agreement with the reported data for compound 2as;

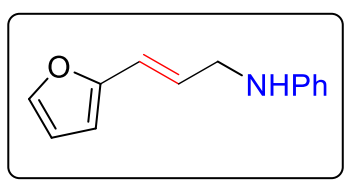

\section{2at: (E)-N-(3-(furan-2-yl)allyl)aniline ${ }^{[29]}$}

97\% yield; white solid; ${ }^{1} \mathrm{H}$ NMR (400 MHz, Chloroform- $d$ ) $\delta 7.22(\mathrm{~d}, J=1.9 \mathrm{~Hz}, 1 \mathrm{H}), 7.08$ (d, $J=15.5 \mathrm{~Hz}, 1 \mathrm{H}), 6.62(\mathrm{t}, J=7.3 \mathrm{~Hz}, 1 \mathrm{H}), 6.53(\mathrm{~d}, J=8.0 \mathrm{~Hz}, 2 \mathrm{H}), 6.32(\mathrm{~d}, J=15.9 \mathrm{~Hz}, 1 \mathrm{H})$, $6.24(\mathrm{t}, J=2.6 \mathrm{~Hz}, 1 \mathrm{H}), 6.15(\mathrm{dt}, J=15.8,5.5 \mathrm{~Hz}, 1 \mathrm{H}), 6.08(\mathrm{~d}, J=3.3 \mathrm{~Hz}, 1 \mathrm{H}), 3.81-3.75$ $(\mathrm{m}, 2 \mathrm{H}), 3.73-3.68(\mathrm{~m}, 1 \mathrm{H}) .{ }^{13} \mathrm{C}$ NMR $(101 \mathrm{MHz}$, Chloroform-d) $\delta$ 152.6, 148.1, 141.9, 129.4, 125.9, 119.8, 117.7, 113.1, 111.4, 107.7, 45.8;

The obtained spectroscopic data were in agreement with the reported data for compound 2at;

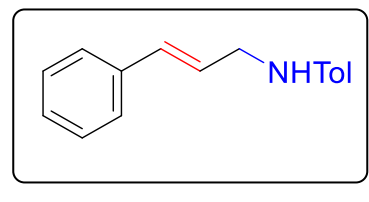

\section{2au: $N$-cinnamyl-4-methylaniline ${ }^{[30]}$}

96\% yield; white solid; ${ }^{1} \mathrm{H}$ NMR (600 MHz, Chloroform- $d$ ) $\delta 7.35$ (d, $\left.J=7.4 \mathrm{~Hz}, 2 \mathrm{H}\right), 7.29$ $(\mathrm{dd}, J=8.4,6.8 \mathrm{~Hz}, 2 \mathrm{H}), 7.24-7.17(\mathrm{~m}, 1 \mathrm{H}), 6.99(\mathrm{~d}, J=8.2 \mathrm{~Hz}, 2 \mathrm{H}), 6.63-6.57$ (m, 3H), $6.32(\mathrm{dt}, J=15.9,5.8 \mathrm{~Hz}, 1 \mathrm{H}), 3.90(\mathrm{dd}, J=5.8,1.7 \mathrm{~Hz}, 2 \mathrm{H}), 3.68(\mathrm{~s}, 1 \mathrm{H}), 2.24(\mathrm{~s}, 3 \mathrm{H}) ;{ }^{13} \mathrm{C}$ 
NMR (151 MHz, Chloroform-d) $\delta$ 145.8, 137.0, 131.4, 129.8, 128.6, 127.5, 127.4, 126.9, 126.4, 113.3, 46.6, 20.5;

The obtained spectroscopic data were in agreement with the reported data for compound $\mathbf{2 a u}$;

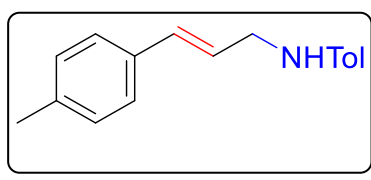

\section{2av: (E)-4-methyl- $N$-(3-(p-tolyl)allyl)aniline}

96\% yield; yellow solid; ${ }^{1} \mathrm{H}$ NMR $(600 \mathrm{MHz}$, Chloroform- $d$ ) $\delta 7.26$ (s, 1H), 7.11 (d, $J=7.9$ $\mathrm{Hz}, 2 \mathrm{H}), 7.00(\mathrm{~d}, J=8.1 \mathrm{~Hz}, 2 \mathrm{H}), 6.62-6.55(\mathrm{~m}, 3 \mathrm{H}), 6.28(\mathrm{dt}, J=15.9,5.9 \mathrm{~Hz}, 1 \mathrm{H}), 3.89$ $(\mathrm{dd}, J=5.9,1.6 \mathrm{~Hz}, 2 \mathrm{H}), 3.68(\mathrm{~s}, 1 \mathrm{H}), 2.33(\mathrm{~s}, 3 \mathrm{H}), 2.24(\mathrm{~s}, 3 \mathrm{H}) ;{ }^{13} \mathrm{C} \mathrm{NMR}(151 \mathrm{MHz}$, Chloroform- $d$ ) $\delta 145.8,137.3,134.1,131.4,129.8,129.3,126.9,126.2,126.2,113.3,46.7,21.2,20.4$; HR ESI-MS (positive ion): $238.1593\left([\mathrm{M}+\mathrm{H}]^{+}\right.$, calcd 238.1596$) \mathrm{m} / \mathrm{z}$

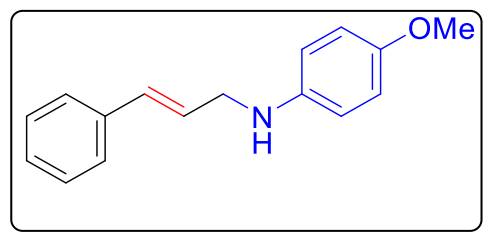

\section{2aw: $N$-cinnamyl-4-methoxyaniline ${ }^{[30]}$}

95\% yield; white solid; ${ }^{1} \mathrm{H}$ NMR (400 MHz, Chloroform- $d$ ) $\delta 7.38$ (d, $J=1.8 \mathrm{~Hz}$, $1 \mathrm{H}), 7.30(\mathrm{t}, J=7.5 \mathrm{~Hz}, 3 \mathrm{H}), 7.23(\mathrm{dd}, J=12.5,5.2 \mathrm{~Hz}, 2 \mathrm{H}), 6.84-6.74(\mathrm{~m}, 3 \mathrm{H})$, $6.69-6.57(\mathrm{~m}, 4 \mathrm{H}), 6.34(\mathrm{dt}, J=15.9,5.8 \mathrm{~Hz}, 1 \mathrm{H}), 3.89(\mathrm{dd}, J=5.9,1.6 \mathrm{~Hz}, 2 \mathrm{H})$, $3.75(\mathrm{~s}, 3 \mathrm{H}) ;{ }^{13} \mathrm{C}$ NMR (101 MHz, Chloroform- $\left.d\right) \delta 152.3,142.3,136.9,131.5$,

128.6, 127.5, 127.4, 126.3, 114.9, 114.5, 55.8, 47.3;

The obtained spectroscopic data were in agreement with the reported data for compound 2aw;

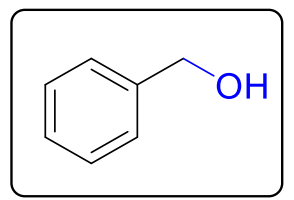

\section{2ba: Phenylmethanol ${ }^{[31]}$}

99\% yield; colorless oil; ${ }^{1} \mathrm{H}$ NMR (400 MHz, Chloroform- $d$ ) $\delta 7.39$ (d, $\left.J=4.5 \mathrm{~Hz}, 4 \mathrm{H}\right), 7.33$ (dt, $J$ $=9.4,4.7 \mathrm{~Hz}, 1 \mathrm{H}), 4.70(\mathrm{~d}, J=1.6 \mathrm{~Hz}, 2 \mathrm{H}), 2.15(\mathrm{~s}, 1 \mathrm{H}) ;{ }^{13} \mathrm{C} \mathrm{NMR}\left(101 \mathrm{MHz}, \mathrm{CDCl}_{3}\right) \delta 140.9$, 128.6, 127.7, 127.0, 65.3;

The obtained spectroscopic data were in agreement with the reported data for compound $\mathbf{2} \mathbf{b a}$;

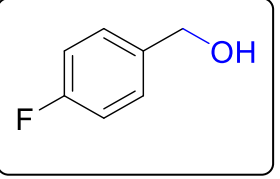

2bb: (4-fluorophenyl)methanol ${ }^{[32]}$

99\% yield; colorless oil; ${ }^{1} \mathrm{H}$ NMR (400 MHz, Chloroform- $d$ ) $\delta 7.33$ (m, 2H), 7.04 (m, 2H), 4.66 (s, $2 \mathrm{H}) ;{ }^{19} \mathrm{~F}$ NMR (376 MHz, Chloroform- $d$ ) $\delta-114.9 ;{ }^{13} \mathrm{C}$ NMR (101 MHz, Chloroform- $d$ ) $\delta 163.5$, $161.1,128.8,128.7,115.5,115.3,64.7$;

The obtained spectroscopic data were in agreement with the reported data for compound $\mathbf{2} \mathbf{b b}$;

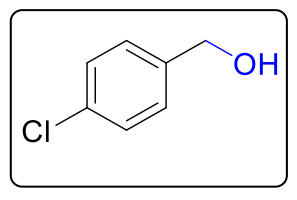

2bc: (4-chlorophenyl)methanol ${ }^{[32]}$

99\% yield; white solid; ${ }^{1} \mathrm{H}$ NMR (600 MHz, Chloroform- $d$ ) $\delta 7.43-7.24(\mathrm{~m}, 4 \mathrm{H}), 4.66(\mathrm{~d}, J=3.6$ $\mathrm{Hz}, 2 \mathrm{H}) ;{ }^{13} \mathrm{C}$ NMR (151 MHz, Chloroform- $d$ ) $\delta$ 139.3, 133.4, 128.7, 128.3, 64.6;

The obtained spectroscopic data were in agreement with the reported data for compound $\mathbf{2 b c}$;

\section{2bd: (4-bromophenyl)methanol ${ }^{[32]}$}

99\% yield; white solid; ${ }^{1} \mathrm{H}$ NMR (400 MHz, Chloroform- $d$ ) $\delta$ 7.59-7.43 (m, 2H), 7.40-7.19 (m, 2H), $4.68(\mathrm{~s}, 2 \mathrm{H}), 1.82(\mathrm{~s}, 1 \mathrm{H}) ;{ }^{13} \mathrm{C}$ NMR (101 MHz, Chloroform- $d$ ) $\delta$ 139.8, 131.6, 128.6, 
The obtained spectroscopic data were in agreement with the reported data for compound $\mathbf{2 b d}$;

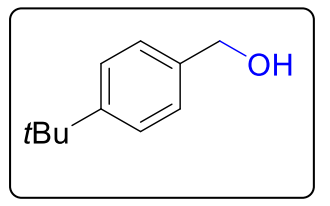

2be: (4-(tert-butyl)phenyl)methanol ${ }^{[33]}$

99\% yield; colorless oil; ${ }^{1} \mathrm{H}$ NMR (400 MHz, Chloroform- $d$ ) $\delta 7.39$ (dd, $J=8.3,1.5 \mathrm{~Hz}, 2 \mathrm{H}$ ), $7.33-7.23(\mathrm{~m}, 2 \mathrm{H}), 4.65(\mathrm{~d}, J=4.0 \mathrm{~Hz}, 2 \mathrm{H}), 1.75(\mathrm{~s}, 1 \mathrm{H}), 1.32(\mathrm{~s}, 9 \mathrm{H}) ;{ }^{13} \mathrm{C}$ NMR $(101 \mathrm{MHz}$, Chloroform- $d$ ) $\delta 150.8,138.0,126.9,125.5,65.2,34.6,31.4$.

The obtained spectroscopic data were in agreement with the reported data for compound $\mathbf{2 b e}$;

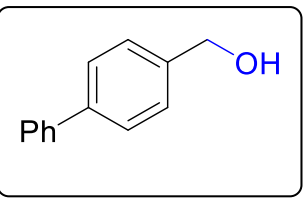

\section{2bf: [1,1'-biphenyl]-4-ylmethano ${ }^{[34]}$}

99\% yield; white solid; ${ }^{1} \mathrm{H}$ NMR (400 MHz, Chloroform- $d$ ) $\delta 7.60$ (d, $\left.J=7.4 \mathrm{~Hz}, 4 \mathrm{H}\right), 7.45$ (d, $J=7.2 \mathrm{~Hz}, 4 \mathrm{H}), 7.37$ (d, $J=7.0 \mathrm{~Hz}, 1 \mathrm{H}), 4.74(\mathrm{~s}, 2 \mathrm{H}), 1.87$ (s, 1H); ${ }^{13} \mathrm{C}$ NMR $(101 \mathrm{MHz}$, Chloroform- $d$ ) $\delta 140.8,140.7,139.9,128.8,127.5,127.4,127.1,65.1$;

The obtained spectroscopic data were in agreement with the reported data for compound $\mathbf{2 b f}$;

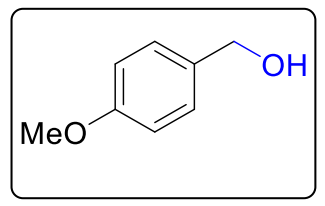

2bg: (4-methoxyphenyl)methano ${ }^{[35]}$

99\% yield; colorless oil; ${ }^{1} \mathrm{H}$ NMR (400 MHz, Chloroform- $d$ ) $\delta$ 7.44-7.17 (m, 2H), 7.01-6.83 (m, 2H), 4.61 (s, 2H), $3.82(\mathrm{~s}, 3 \mathrm{H}), 2.12(\mathrm{~s}, 1 \mathrm{H}) ;{ }^{13} \mathrm{C}$ NMR (101 MHz, Chloroform- $d$ ) $\delta$ 159.1, 133.2, 128.7, 113.9, 64.9, 55.3.

The obtained spectroscopic data were in agreement with the reported data for compound $\mathbf{2} \mathbf{b g}$;

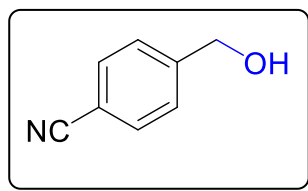

\section{2bh: 4-(hydroxymethyl)benzonitrile ${ }^{[36]}$}

96\% yield; white solid; ${ }^{1} \mathrm{H}$ NMR (400 MHz, Chloroform- $d$ ) $\delta 7.63$ (d, $\left.J=7.9 \mathrm{~Hz}, 2 \mathrm{H}\right), 7.47$ (d, $J=8.0 \mathrm{~Hz}, 2 \mathrm{H}), 4.77$ (s, 2H), 2.64 (s, 1H); ${ }^{13} \mathrm{C}$ NMR (101 MHz, Chloroform- $d$ ) $\delta 146.5,132.3$, 127.0, 118.9, 110.9, 64.1;

The obtained spectroscopic data were in agreement with the reported data for compound $\mathbf{2} \mathbf{b h}$;

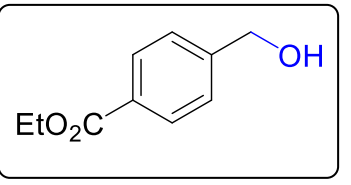

\section{2bi: Ethyl 4-(hydroxymethyl)benzoate ${ }^{[37]}$}

95\% yield; white solid; ${ }^{1} \mathrm{H}$ NMR (400 MHz, Chloroform- $d$ ) $\delta 8.14-7.99(\mathrm{~m}, 2 \mathrm{H}), 7.45$ (d, $J=$ $8.1 \mathrm{~Hz}, 2 \mathrm{H}), 4.79$ (s, 2H), 4.39 (q, $J=7.1 \mathrm{~Hz}, 2 \mathrm{H}), 2.03(\mathrm{~d}, J=8.1 \mathrm{~Hz}, 1 \mathrm{H}), 1.42(\mathrm{t}, J=7.1$ $\mathrm{Hz}, 3 \mathrm{H}) ;{ }^{13} \mathrm{C}$ NMR (101 MHz, Chloroform- $d$ ) $\delta 166.5,145.9,129.8,126.4,64.7,61.0,14.4$;

The obtained spectroscopic data were in agreement with the reported data for compound $\mathbf{2 b i}$; 


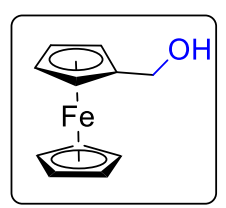

\section{2bj: Ferrocenemethanol ${ }^{[38]}$}

98\% yield; organge solid; ${ }^{1} \mathrm{H}$ NMR (400 MHz, Chloroform- $d$ ) $\delta 4.32(\mathrm{~s}, 2 \mathrm{H}), 4.23(\mathrm{t}, J=1.9 \mathrm{~Hz}, 2 \mathrm{H})$, 4.17 (dd, $J=2.9,1.4 \mathrm{~Hz}, 7 \mathrm{H}), 1.68$ (brs, $1 \mathrm{H}) ;{ }^{13} \mathrm{C}$ NMR (101 MHz, Chloroform- $d$ ) $\delta 88.4,68.3,68.3$, $67.9,60.8$.

The obtained spectroscopic data were in agreement with the reported data for compound $\mathbf{2} \mathbf{b j}$;

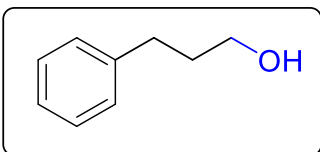

$125.9,62.3,34.2,32.1$

2bk: 3-phenylpropan-1-o[ ${ }^{[39]}$

92\% yield; colorless oil; ${ }^{1} \mathrm{H}$ NMR (400 MHz, Chloroform- $d$ ) $\delta 7.31-7.26(\mathrm{~m}, 2 \mathrm{H}), 7.19$ (ddd, $J=8.5,5.2,2.9 \mathrm{~Hz}, 3 \mathrm{H}), 3.68(\mathrm{t}, J=6.4 \mathrm{~Hz}, 2 \mathrm{H}), 2.71(\mathrm{dd}, J=8.7,6.8 \mathrm{~Hz}, 2 \mathrm{H}), 2.00-1.81$ $(\mathrm{m}, 2 \mathrm{H}), 1.69$ (s, 1H); ${ }^{13} \mathrm{C}$ NMR (101 MHz, Chloroform- $\left.d\right) \delta 141.8,128.5,128.5,128.5,128.4$,

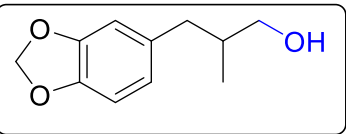

2bl: 3-(benzo[d][1,3] dioxol-5-yl)-2-methylpropan-1-ol ${ }^{[40]}$

99\% yield; white solid; ${ }^{1} \mathrm{H}$ NMR (400 MHz, Chloroform- $d$ ) $\delta 6.72(\mathrm{~d}, J=7.9 \mathrm{~Hz}, 1 \mathrm{H}), 6.67$ $(\mathrm{d}, J=1.7 \mathrm{~Hz}, 1 \mathrm{H}), 6.61(\mathrm{dd}, J=7.8,1.7 \mathrm{~Hz}, 1 \mathrm{H}), 5.91(\mathrm{~s}, 2 \mathrm{H}), 3.48$ (qd, $J=10.6,5.9 \mathrm{~Hz}$,

2H), 2.67 (dd, $J=13.6,6.3 \mathrm{~Hz}, 1 \mathrm{H}), 2.34$ (dd, $J=13.6,8.0 \mathrm{~Hz}, 1 \mathrm{H}), 1.88(\mathrm{dp}, J=7.8,6.3 \mathrm{~Hz}, 1 \mathrm{H}), 1.71(\mathrm{~s}, 1 \mathrm{H}), 0.90$ (d, $J=6.8 \mathrm{~Hz}, 3 \mathrm{H}) ;{ }^{13} \mathrm{C}$ NMR $(101 \mathrm{MHz}$, Chloroform- $d$ ) $\delta 147.5,145.7,134.4,121.9,109.5,108.1,100.8,67.5,39.4,37.9$, 16.4 .

The obtained spectroscopic data were in agreement with the reported data for compound $\mathbf{2} \mathbf{b l}$;

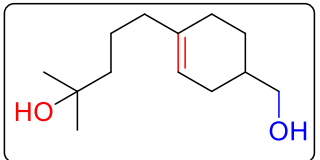

2bm: 5-(4-(hydroxymethyl)cyclohex-1-en-1-yl)-2-methylpentan-2-ol ${ }^{[41]}$

99\% yield; colorless oil; ${ }^{1} \mathrm{H}$ NMR (400 MHz, Chloroform- $d$ ) $\delta 5.42-5.36(\mathrm{~m}, 1 \mathrm{H}), 3.55$ (dt, $J=$ 9.1, $5.9 \mathrm{~Hz}, 2 \mathrm{H}), 2.09(\mathrm{~s}, 1 \mathrm{H}), 2.02-1.92(\mathrm{~m}, 5 \mathrm{H}), 1.84(\mathrm{ddt}, J=11.8,5.6,3.3 \mathrm{~Hz}, 1 \mathrm{H}), 1.75$ $(\mathrm{dd}, J=4.2,2.4 \mathrm{~Hz}, 1 \mathrm{H}), 1.75-1.65(\mathrm{~m}, 1 \mathrm{H}), 1.60(\mathrm{~s}, 4 \mathrm{H}), 1.52-1.38(\mathrm{~m}, 3 \mathrm{H}), 1.27(\mathrm{~s}, 6 \mathrm{H}) ;{ }^{13} \mathrm{C} \mathrm{NMR}(100 \mathrm{MHz}$, Chloroform- $d$ ) $\delta 137.7,119.8,71.1,67.6,43.5,38.0,36.3,29.2,28.2,27.6,25.6,22.4$.

The obtained spectroscopic data were in agreement with the reported data for compound $\mathbf{2} \mathbf{b m}$;

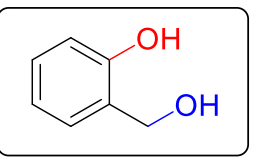

\section{2bn: 2-(hydroxymethyl)phenol ${ }^{[42]}$}

95\% yield; white solid; ${ }^{1} \mathrm{H}$ NMR (400 MHz, Chloroform- $d$ ) $\delta$ 7.24-7.17 (m, 1H), $7.04(\mathrm{~d}, J=6.9$ $\mathrm{Hz}, 1 \mathrm{H}), 6.91-6.83(\mathrm{~m}, 2 \mathrm{H}), 4.85(\mathrm{~s}, 2 \mathrm{H}), 1.26(\mathrm{~s}, 1 \mathrm{H}) ;{ }^{13} \mathrm{C}$ NMR (101 MHz, Chloroform- $\left.d\right) \delta 156.0$,

$129.5,127.9,124.8,120.1,116.5,64.5$;

The obtained spectroscopic data were in agreement with the reported data for compound $\mathbf{2} \mathbf{b n}$;

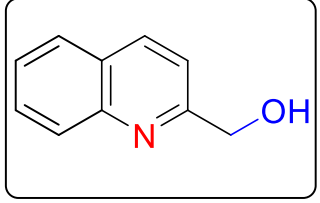




\section{2bo: quinolin-2-ylmethanol ${ }^{[43]}$}

95\% yield; pale yellow solid; ${ }^{1} \mathrm{H}$ NMR $(400 \mathrm{MHz}$, Chloroform- $d) \delta 8.10(\mathrm{~d}, J=8.3 \mathrm{~Hz}, 1 \mathrm{H}), 7.86(\mathrm{~d}, J=8.0 \mathrm{~Hz}, 1 \mathrm{H})$, 7.75 (t, $J=7.4 \mathrm{~Hz}, 2 \mathrm{H}), 7.58(\mathrm{~d}, J=7.4 \mathrm{~Hz}, 1 \mathrm{H}), 7.29$ (d, $J=8.0 \mathrm{~Hz}, 1 \mathrm{H}), 4.95(\mathrm{~s}, 2 \mathrm{H}), 4.57$ (brs, $1 \mathrm{H}) ;{ }^{13} \mathrm{C} \mathrm{NMR}(101$ MHz, Chloroform- $d$ ) $\delta 136.9,129.8,128.7,127.7,126.4,118.3,64.1$.

The obtained spectroscopic data were in agreement with the reported data for compound $\mathbf{2 b o}$;

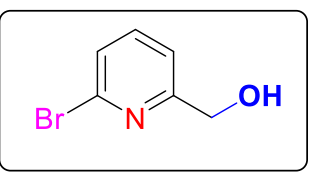

\section{2bp: (6-bromopyridin-2-yl)methanol ${ }^{[44]}$}

85\% yield; pale yellow solid; ${ }^{1} \mathrm{H}$ NMR (400 MHz, Chloroform- $d$ ) $\delta 7.55$ (t, $J=7.7 \mathrm{~Hz}, 1 \mathrm{H}$ ), $7.38(\mathrm{~d}, J=7.9 \mathrm{~Hz}, 1 \mathrm{H}), 7.32(\mathrm{~d}, J=7.6 \mathrm{~Hz}, 1 \mathrm{H}), 4.75(\mathrm{~s}, 2 \mathrm{H}), 3.68(\mathrm{~s}, 1 \mathrm{H}) ;{ }^{13} \mathrm{C}$ NMR $(101$ MHz, Chloroform-d) $\delta 161.5,141.3,139.2,126.6,119.4,64.2$;

The obtained spectroscopic data were in agreement with the reported data for compound $\mathbf{2 b p}$;

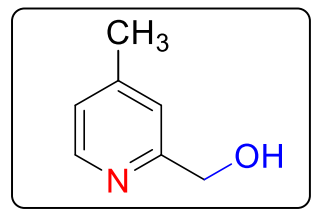

\section{2bq: (4-methylpyridin-2-yl)methanol ${ }^{[45]}$}

95\% yield; colorless oil; ${ }^{1} \mathrm{H}$ NMR (400 MHz, Chloroform- $d$ ) $\delta 8.37(\mathrm{~d}, J=5.1 \mathrm{~Hz}, 1 \mathrm{H}), 7.08$ (s, $1 \mathrm{H}), 7.01(\mathrm{~d}, J=5.1 \mathrm{~Hz}, 1 \mathrm{H}), 4.70(\mathrm{~s}, 2 \mathrm{H}), 2.34(\mathrm{~s}, 3 \mathrm{H}) ;{ }^{13} \mathrm{C}$ NMR $(101 \mathrm{MHz}$, Chloroform-d) $\delta$ 159.0, 148.2, 148.1, 123.4, 121.4, 64.2, 21.1;

The obtained spectroscopic data were in agreement with the reported data for compound $\mathbf{2 b q}$;

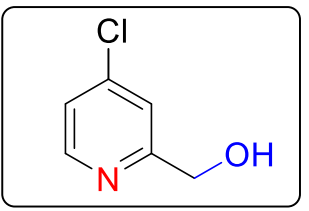

2br: (4-chloropyridin-2-yl)methanol ${ }^{[46]}$

90\% yield; white solid; ${ }^{1} \mathrm{H}$ NMR (400 MHz, Chloroform- $d$ ) $\delta 8.43(\mathrm{~d}, J=5.4 \mathrm{~Hz}, 1 \mathrm{H}), 7.33(\mathrm{~d}, J$ $=2.1 \mathrm{~Hz}, 1 \mathrm{H}), 7.21(\mathrm{dd}, J=5.7,1.9 \mathrm{~Hz}, 1 \mathrm{H}), 4.74(\mathrm{~s}, 2 \mathrm{H}), 3.81(\mathrm{~s}, 1 \mathrm{H}) ;{ }^{13} \mathrm{C} \mathrm{NMR}(101 \mathrm{MHz}$, Chloroform- $d$ ) $\delta 161.2,149.5,144.9,122.8,120.9,64.1$;

The obtained spectroscopic data were in agreement with the reported data for compound $\mathbf{2 b r}$;

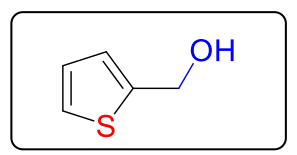

125.6, 125.5, 59.9;

\section{2bs: thiophen-2-ylmethanol ${ }^{[47]}$}

96\% yield; yellow oil; ${ }^{1} \mathrm{H}$ NMR (400 MHz, Chloroform- $d$ ) $\delta 7.29$ (dd, $\left.J=4.9,1.4 \mathrm{~Hz}, 1 \mathrm{H}\right), 7.06-$ 6.95 (m, 2H), 4.82 (s, 2H), $2.48(\mathrm{~s}, 1 \mathrm{H}) ;{ }^{13} \mathrm{C}$ NMR (101 MHz, Chloroform-d) $\delta 144.1,126.88$,

The obtained spectroscopic data were in agreement with the reported data for compound $\mathbf{2 b s}$;

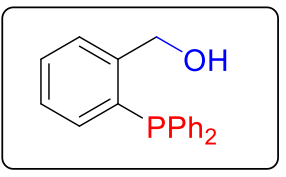

2bt: (2-(diphenylphosphaneyl)phenyl)methanol ${ }^{[48]}$

87\% yield; white solid; ${ }^{1} \mathrm{H}$ NMR (400 MHz, Chloroform- $d$ ) $\delta 7.55$ (dd, $J=7.5,4.4 \mathrm{~Hz}, 1 \mathrm{H}$ ), $7.44-7.34$ (m, 7H), 7.30 (ddd, $J=7.9,5.6,2.4 \mathrm{~Hz}, 4 \mathrm{H}), 7.24(\mathrm{t}, J=7.5 \mathrm{~Hz}, 1 \mathrm{H}), 6.94$ (dd, $J=7.5$, $4.7 \mathrm{~Hz}, 1 \mathrm{H}), 4.86(\mathrm{~d}, J=4.8 \mathrm{~Hz}, 2 \mathrm{H}), 2.16(\mathrm{~s}, 1 \mathrm{H}) ;{ }^{31} \mathrm{P} \mathrm{NMR}\left(162 \mathrm{MHz}, \mathrm{CDCl}_{3}\right) \delta-16.3 ;{ }^{13} \mathrm{C}$ NMR (101 MHz, Chloroform-d) $\delta 136.0,135.9,134.0,133.8,133.5,129.3,129.0,128.7,128.6,128.1,128.0,127.9$, 64.0, 63.8;

The obtained spectroscopic data were in agreement with the reported data for compound $\mathbf{2 b t}$; 


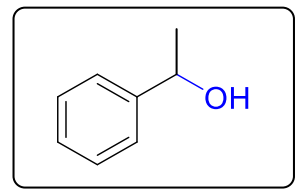

2bu: 1-phenylethan-1-ol ${ }^{[49]}$

99\% yield; colorless oil; ${ }^{1} \mathrm{H}$ NMR (400 MHz, Chloroform- $d$ ) $\delta$ 7.41-7.33 (m, 4H), 7.32-7.25 (m, $1 \mathrm{H}), 4.90(\mathrm{q}, J=6.5 \mathrm{~Hz}, 1 \mathrm{H}), 2.52(\mathrm{~s}, 1 \mathrm{H}), 1.50(\mathrm{~d}, J=6.5 \mathrm{~Hz}, 3 \mathrm{H}) ;{ }^{13} \mathrm{C}$ NMR $(101 \mathrm{MHz}$, Chloroform- $d$ ) $\delta 145.9,128.4,127.3,125.3,70.2,25.1$;

The obtained spectroscopic data were in agreement with the reported data for compound $\mathbf{2} \mathbf{b u}$;

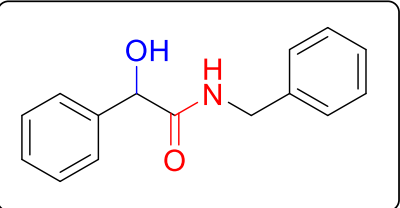

2bv: $N$-benzyl-2-hydroxy-2-phenylacetamide ${ }^{[50]}$

95\% yield; white solid; ${ }^{1} \mathrm{H}$ NMR (400 MHz, Chloroform- $d$ ) $\delta 7.33$ (ddd, $J=15.8,9.4,4.8$ $\mathrm{Hz}, 8 \mathrm{H}), 7.22-7.11(\mathrm{~m}, 2 \mathrm{H}), 6.94(\mathrm{t}, J=5.8 \mathrm{~Hz}, 1 \mathrm{H}), 4.98(\mathrm{~s}, 1 \mathrm{H}), 4.40-4.28(\mathrm{~m}, 3 \mathrm{H})$; ${ }^{13} \mathrm{C}$ NMR (101 MHz, Chloroform- $d$ ) $\delta$ 172.6, 139.6, 137.7, 128.7, 128.5, 127.6, 126.8, 74.1, 43.3;

The obtained spectroscopic data were in agreement with the reported data for compound $\mathbf{2} \mathbf{b v}$;

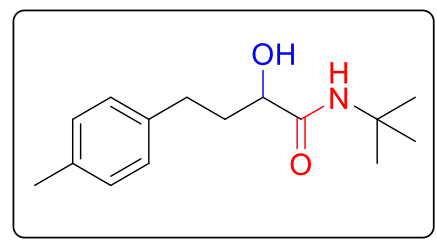

\section{2bw: $N$-(tert-butyl)-2-hydroxy-4-(p-tolyl)butanamide}

93\% yield; white solid; ${ }^{1} \mathrm{H}$ NMR (400 MHz, Chloroform- $d$ ) $\delta 7.12(\mathrm{~s}, 4 \mathrm{H}), 6.32(\mathrm{~s}, 1 \mathrm{H})$, 4.10-3.93 (m, 1H), 3.21 (d, $J=5.1 \mathrm{~Hz}, 1 \mathrm{H}), 2.73$ (t, $J=7.9 \mathrm{~Hz}, 2 \mathrm{H}), 2.33$ (s, 3H), 2.11 (dtd, $J=16.8,8.1,3.5 \mathrm{~Hz}, 1 \mathrm{H}), 1.93(\mathrm{~d}, J=7.5 \mathrm{~Hz}, 1 \mathrm{H}), 1.38(\mathrm{~s}, 9 \mathrm{H}) ;{ }^{13} \mathrm{C}$ NMR $(101$ MHz, Chloroform- $d$ ) $\delta$ 173.0, 138.3, 135.5, 129.2, 128.4, 71.6, 51.0, 36.8, 30.8, 28.8, 21.0; HRMS(ESI): $[\mathrm{M}+\mathrm{H}]^{+} \mathrm{C}_{15} \mathrm{H}_{24} \mathrm{O}_{2} \mathrm{~N}$, calcd: 250.1802 , found 250.1800 .

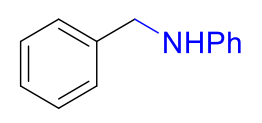

\section{2bx: $N$-benzylaniline ${ }^{[51]}$}

96\% yield; white solid; ${ }^{1} \mathrm{H}$ NMR (400 MHz, Chloroform- $d$ ) $\delta 7.32-7.15(\mathrm{~m}, 5 \mathrm{H}), 7.15-7.04$ (m, 2H), $6.63(\mathrm{tt}, J=7.3,1.2 \mathrm{~Hz}, 1 \mathrm{H}), 6.58-6.50(\mathrm{~m}, 2 \mathrm{H}), 4.23(\mathrm{~s}, 2 \mathrm{H}), 3.93(\mathrm{~s}, 1 \mathrm{H}) .{ }^{13} \mathrm{C}$ NMR (101 MHz, Chloroform- $d$ ) $\delta$ 148.2, 139.5, 129.3, 128.7, 127.6, 127.3, 117.6, 112.9, 48.4;

The obtained spectroscopic data were in agreement with the reported data for compound $\mathbf{2 b x}$;

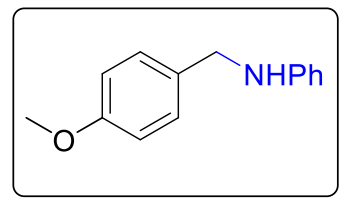

\section{2by: $N$-(4-methoxybenzyl)aniline ${ }^{[52]}$}

96\% yield; white solid; ${ }^{1} \mathrm{H}$ NMR (400 MHz, Chloroform- $d$ ) $\delta 7.28$ (d, $\left.J=8.3 \mathrm{~Hz}, 2 \mathrm{H}\right), 7.22$ $7.13(\mathrm{~m}, 2 \mathrm{H}), 6.88(\mathrm{dd}, J=8.9,2.8 \mathrm{~Hz}, 2 \mathrm{H}), 6.76-6.66(\mathrm{~m}, 1 \mathrm{H}), 6.66-6.59(\mathrm{~m}, 2 \mathrm{H}), 4.24(\mathrm{~s}$, 2H), $3.94(\mathrm{~s}, 1 \mathrm{H}), 3.79$ (d, $J=1.2 \mathrm{~Hz}, 3 \mathrm{H}) ;{ }^{13} \mathrm{C}$ NMR (101 MHz, Chloroform-d) $\delta$ 158.9, 148.2,

131.4, 129.3, 128.8, 117.5, 114.1, 112.9, 55.3, 47.8;

The obtained spectroscopic data were in agreement with the reported data for compound $\mathbf{2 b y}$;

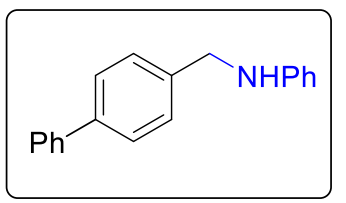

\section{2bz: $N$-([1,1'-biphenyl]-4-ylmethyl)aniline ${ }^{[53]}$}

96\% yield; white solid; ${ }^{1} \mathrm{H}$ NMR (400 MHz, Chloroform- $d$ ) $\delta 7.66-7.62(\mathrm{~m}, 4 \mathrm{H}), 7.51-7.48$ $(\mathrm{m}, 4 \mathrm{H}), 7.40$ (t, J = 7.8 Hz, 1H), $7.26-7.23(\mathrm{~m}, 2 \mathrm{H}), 6.72(\mathrm{t}, \mathrm{J}=7.3 \mathrm{~Hz}, 1 \mathrm{H}), 6.67$ (d, J = 7.8 
$\mathrm{Hz}, 2 \mathrm{H}), 4.37$ (s, 2H), 4.03 (br s, 1H); ${ }^{13} \mathrm{C}$ NMR (101 MHz, Chloroform-d) $\delta 148.2,140.9,140.3,138.6,129.3,128.8$, 128.0, 127.4, 127.3, 127.1, 117.7, 112.9, 48.0;

The obtained spectroscopic data were in agreement with the reported data for compound $\mathbf{2 b z}$;

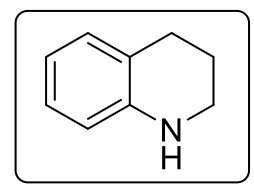

2ca: 1,2,3,4-tetrahydroquinoline ${ }^{[54]}$

Yield: 99\%; ${ }^{1} \mathrm{H}$ NMR (400 MHz, Chloroform- $d$ ) $\delta 7.01-6.92(\mathrm{~m}, 2 \mathrm{H}), 6.61(\mathrm{td}, J=7.4,1.2 \mathrm{~Hz}, 1 \mathrm{H})$, $6.48(\mathrm{dd}, J=8.0,1.1 \mathrm{~Hz}, 1 \mathrm{H}), 3.74(\mathrm{~s}, 1 \mathrm{H}), 3.34-3.27(\mathrm{~m}, 2 \mathrm{H}), 2.77(\mathrm{t}, J=6.4 \mathrm{~Hz}, 2 \mathrm{H}), 1.95$ (dtdd, $J=8.8,6.3,4.2,2.3 \mathrm{~Hz}, 2 \mathrm{H}) ;{ }^{13} \mathrm{C}$ NMR $(101 \mathrm{MHz}$, Chloroform-d) $\delta 144.8,129.5,126.7,121.5,117.0,114.2,42.0,27.0$, 22.2;

The obtained spectroscopic data were in agreement with the reported data for this compound 2ca;

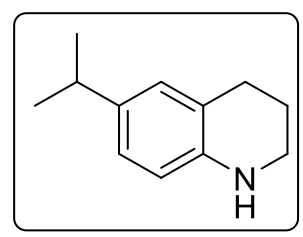

\section{2cb: 6-isopropyl-1,2,3,4-tetrahydroquinoline $\mathrm{e}^{[55]}$}

Yield: 99\%; ${ }^{1} \mathrm{H}$ NMR (600 MHz, Chloroform- $\left.d\right) \delta 6.86-6.79(\mathrm{~m}, 2 \mathrm{H}), 6.43(\mathrm{~d}, J=8.0 \mathrm{~Hz}, 1 \mathrm{H})$, $3.53(\mathrm{~s}, 1 \mathrm{H}), 3.30-3.24(\mathrm{~m}, 2 \mathrm{H}), 2.75(\mathrm{td}, J=6.7,4.1 \mathrm{~Hz}, 3 \mathrm{H}), 1.93(\mathrm{dt}, J=10.9,6.3 \mathrm{~Hz}, 2 \mathrm{H})$, $1.19(\mathrm{~d}, J=6.9 \mathrm{~Hz}, 6 \mathrm{H}) ;{ }^{13} \mathrm{C}$ NMR $(151 \mathrm{MHz}$, Chloroform- $d) \delta 142.8,137.7,127.5,124.7,121.4$, 114.5, 42.2, 33.3, 27.1, 24.3, 22.5;

The obtained spectroscopic data were in agreement with the reported data for this compound $\mathbf{2 c b}$;

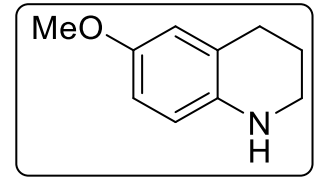

\section{2cc: 6-methoxy-1,2,3,4-tetrahydroquinoline ${ }^{[56]}$}

Yield: 90\%; ${ }^{1} \mathrm{H}$ NMR (400 MHz, Chloroform- $d$ ) $\delta 6.63-6.53(\mathrm{~m}, 2 \mathrm{H}), 6.45(\mathrm{~d}, J=8.5 \mathrm{~Hz}, 1 \mathrm{H})$, $3.72(\mathrm{~s}, 3 \mathrm{H}), 3.28-3.21(\mathrm{~m}, 2 \mathrm{H}), 2.75(\mathrm{t}, J=6.5 \mathrm{~Hz}, 2 \mathrm{H}), 1.98-1.87(\mathrm{~m}, 2 \mathrm{H}) ;{ }^{13} \mathrm{C}$ NMR $(101$ MHz, Chloroform- $d$ ) $\delta 151.8,138.9,122.9,115.6,114.9,112.9,55.8,42.4,27.2,22.5$;

The obtained spectroscopic data were in agreement with the reported data for this compound 2cc;

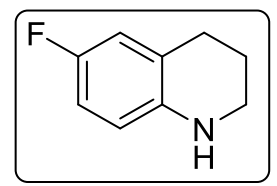

\section{2cd: 6-fluoro-1,2,3,4-tetrahydroquinoline ${ }^{[57]}$}

Yield: 98\%; ${ }^{1} \mathrm{H}$ NMR (400 MHz, Chloroform- $d$ ) $\delta 6.68$ (ddd, $J=8.7,4.2,1.7 \mathrm{~Hz}, 4 \mathrm{H}$ ), 6.40 (dd, $J$ $=9.5,4.8 \mathrm{~Hz}, 2 \mathrm{H}), 3.32-3.23(\mathrm{~m}, 4 \mathrm{H}), 2.74(\mathrm{t}, J=6.5 \mathrm{~Hz}, 4 \mathrm{H}), 1.99-1.87(\mathrm{~m}, 4 \mathrm{H}), 1.28(\mathrm{~s}, 1 \mathrm{H})$, $1.26(\mathrm{~s}, 1 \mathrm{H}) ;{ }^{13} \mathrm{C}$ NMR $(101 \mathrm{MHz}$, Chloroform- $d) \delta 154.3,140.9,122.9,122.8,115.7,115.5,115.0,114.9,113.3,113.1$, 42.1, 27.0, 22.0; ${ }^{19} \mathrm{~F}$ NMR (376 MHz, Chloroform- $d$ ) $\delta$-128.3;

The obtained spectroscopic data were in agreement with the reported data for this compound $\mathbf{2 c d}$.

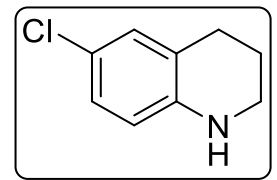

2ce: 6-chloro-1,2,3,4-tetrahydroquinoline ${ }^{[54]}$

Yield: 98\%; ${ }^{1} \mathrm{H}$ NMR (400 MHz, Chloroform- $d$ ) $\delta 7.03-6.74(\mathrm{~m}, 2 \mathrm{H}), 6.52-6.17(\mathrm{~m}, 1 \mathrm{H}), 3.84(\mathrm{~s}$, 
$1 \mathrm{H}), 3.27(\mathrm{~d}, J=6.8 \mathrm{~Hz}, 2 \mathrm{H}), 2.71(\mathrm{t}, J=6.4 \mathrm{~Hz}, 2 \mathrm{H}), 1.95-1.81(\mathrm{~m}, 2 \mathrm{H}) ;{ }^{13} \mathrm{C}$ NMR $(101 \mathrm{MHz}$, Chloroform- $d) \delta 143.3$, $129.03,126.5,122.9,121.1,115.1,41.9,26.9,21.8$;

The obtained spectroscopic data were in agreement with the reported data for this compound 2 ce.

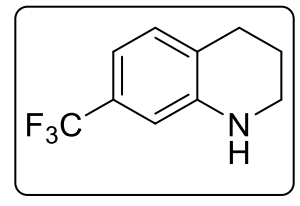

\section{2cf: 7-(trifluoromethyl)-1,2,3,4-tetrahydroquinoline ${ }^{[58]}$}

Yield: 95\%; ${ }^{1} \mathrm{H}$ NMR (600 MHz, Chloroform- $\left.d\right) \delta 7.00(\mathrm{~d}, J=7.8 \mathrm{~Hz}, 1 \mathrm{H}), 6.80(\mathrm{~d}, J=7.9 \mathrm{~Hz}$, 1H), 6.65 (s, 1H), 3.99 (s, 1H), $3.37-3.29$ (m, 2H), 2.77 (t, $J=6.5 \mathrm{~Hz}, 2 \mathrm{H}), 1.93$ (p, J = 6.1 Hz, $2 \mathrm{H}) ;{ }^{19} \mathrm{~F}$ NMR (565 MHz, Chloroform- $d$ ) $\delta-62.64 ;{ }^{13} \mathrm{C}$ NMR $(151 \mathrm{MHz}$, Chloroform- $d$ ) $\delta 144.8$, 129.7, 129.2, 129.0, 124.8, 123.5, 113.1, 113.00, 113.0, 110.2, 110.22, 110.2, 41.8, 27.0, 21.5;

The obtained spectroscopic data were in agreement with the reported data for this compound $2 \mathrm{cf}$.

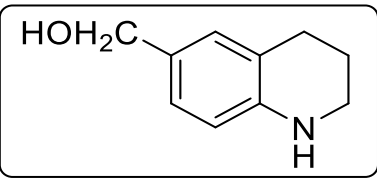

\section{2cg: 6-(hydroperoxymethyl)-1,2,3,4-tetrahydroquinoline ${ }^{[59]}$}

Yield: 96\%; ${ }^{1} \mathrm{H}$ NMR (400 MHz, Chloroform- $d$ ) $\delta 6.96(\mathrm{dt}, J=4.5,2.5 \mathrm{~Hz}, 2 \mathrm{H}), 6.45(\mathrm{~d}, J$ $=8.6 \mathrm{~Hz}, 1 \mathrm{H}), 4.49(\mathrm{~s}, 2 \mathrm{H}), 3.36-3.23(\mathrm{~m}, 2 \mathrm{H}), 2.76(\mathrm{t}, J=6.4 \mathrm{~Hz}, 2 \mathrm{H}), 2.00-1.88(\mathrm{~m}$, $2 \mathrm{H}) ;{ }^{13} \mathrm{C}$ NMR (101 MHz, Chloroform- $d$ ) $\delta$ 144.5, 129.4, 129.2, 126.4, 121.5, 114.2, 65.5, 42.0, 27.0, 22.1;

The obtained spectroscopic data were in agreement with the reported data for this compound $\mathbf{2} \mathbf{c g}$;

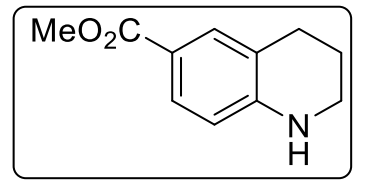

\section{2ch: methyl 1,2,3,4-tetrahydroquinoline-6-carboxylate ${ }^{[57]}$}

Yield: $80 \%$; ${ }^{1} \mathrm{H}$ NMR $(400 \mathrm{MHz}$, Chloroform- $d$ ) $\delta 7.68-7.60(\mathrm{~m}, 2 \mathrm{H}), 6.39(\mathrm{~d}, J=8.9 \mathrm{~Hz}$, $1 \mathrm{H}), 4.33(\mathrm{~s}, 1 \mathrm{H}), 3.83(\mathrm{~s}, 3 \mathrm{H}), 3.38-3.31(\mathrm{~m}, 2 \mathrm{H}), 2.76(\mathrm{t}, J=6.3 \mathrm{~Hz}, 2 \mathrm{H}), 1.92(\mathrm{dtd}, J=$ 8.8, 6.8, 6.2, $5.0 \mathrm{~Hz}, 2 \mathrm{H}) ;{ }^{13} \mathrm{C}$ NMR (101 MHz, Chloroform- $d$ ) $\delta$ 167.6, 148.8, 131.3, 129.1, 119.9, 117.4, 112.7, 51.5, 41.7, 26.9, 21.4;

The obtained spectroscopic data were in agreement with the reported data for this compound $\mathbf{2} \mathbf{c h}$;

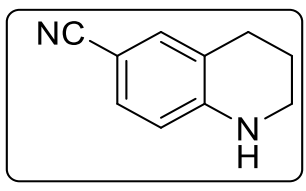

\section{2ci: 1,2,3,4-tetrahydroquinoline-6-carbonitrile ${ }^{[57]}$}

Yield: 76\% (s/c = 100); ${ }^{1} \mathrm{H}$ NMR $(400 \mathrm{MHz}$, Chloroform- $d) \delta 7.24-7.13(\mathrm{~m}, 2 \mathrm{H}), 6.39(\mathrm{~d}, J=$ $8.2 \mathrm{~Hz}, 1 \mathrm{H}), 4.41(\mathrm{~s}, 1 \mathrm{H}), 3.36(\mathrm{td}, J=5.7,2.3 \mathrm{~Hz}, 2 \mathrm{H}), 2.73(\mathrm{t}, J=6.3 \mathrm{~Hz}, 2 \mathrm{H}), 2.00-1.85(\mathrm{~m}$, $2 \mathrm{H}) .{ }^{13} \mathrm{C}$ NMR (101 MHz, Chloroform- $d$ ) $\delta$ 148.3, 133.2, 131.3, 120.9, 120.9, 113.2, 97.7, 41.6, 26.7, 21.0;

The obtained spectroscopic data were in agreement with the reported data for this compound $\mathbf{2 c i}$;

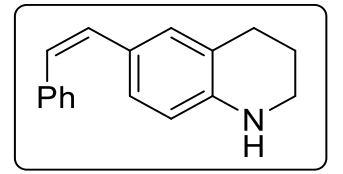

\section{2cj: (Z)-6-styryl-1,2,3,4-tetrahydroquinoline}

Yield: $85 \%$; ${ }^{1} \mathrm{H}$ NMR $(600 \mathrm{MHz}$, Chloroform- $d$ ) $\delta 7.35-7.31(\mathrm{~m}, 2 \mathrm{H}), 7.23(\mathrm{dd}, J=14.8,7.4$ $\mathrm{Hz}, 2 \mathrm{H}), 7.16$ (t, $J=7.3 \mathrm{~Hz}, 1 \mathrm{H}), 6.89(\mathrm{~m}, 2 \mathrm{H}), 6.42$ (d, $J=12.2 \mathrm{~Hz}, 1 \mathrm{H}), 6.39$ (d, $J=12.2 \mathrm{~Hz}$, $1 \mathrm{H}), 6.28(\mathrm{~d}, J=8.0 \mathrm{~Hz}, 1 \mathrm{H}), 3.86(\mathrm{~s}, 1 \mathrm{H}), 3.28(\mathrm{t}, J=6.4 \mathrm{~Hz}, 2 \mathrm{H}), 2.64(\mathrm{t}, J=6.4 \mathrm{~Hz}, 2 \mathrm{H}), 1.90$ 
(m, 2H); ${ }^{13} \mathrm{C}$ NMR (151 MHz, Chloroform- $d$ ) $\delta$ 144.0, 138.3, 130.5, 130.4, 128.8, 128.1, 127.6, 126.6, 126.5, 125.8, 120.8, 113.6, 42.0, 26.9, 22.1; HR ESI-MS (positive ion): $236.1443\left([\mathrm{M}+\mathrm{H}]^{+}\right.$, calcd 236.1440) m/z.

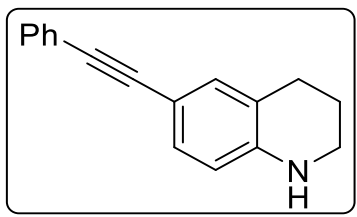

\section{2ck: 6-(phenylethynyl)-1,2,3,4-tetrahydroquinoline ${ }^{[60]}$}

Yield: 80\%; ${ }^{1} \mathrm{H}$ NMR (600 MHz, Chloroform- $d$ ) $\delta 7.50-7.43(\mathrm{~m}, 2 \mathrm{H}), 7.39-7.17(\mathrm{~m}$, 4H), $7.16-7.09(\mathrm{~m}, 2 \mathrm{H}), 6.40(\mathrm{~d}, J=8.7 \mathrm{~Hz}, 1 \mathrm{H}), 4.03(\mathrm{~s}, 1 \mathrm{H}), 3.35-3.27(\mathrm{~m}, 2 \mathrm{H}), 2.75$ $(\mathrm{t}, J=6.4 \mathrm{~Hz}, 2 \mathrm{H}), 1.97-1.90(\mathrm{~m}, 2 \mathrm{H}) ;{ }^{13} \mathrm{C}$ NMR $(151 \mathrm{MHz}$, Chloroform- $d$ ) $\delta 145.0$,

$132.9,131.3,130.5,128.2,127.4,124.2,121.0,113.6,110.5,90.8,86.8,41.9,26.8,21.9$.

The obtained spectroscopic data were in agreement with the reported data for this compound $\mathbf{2} \mathbf{c j}$;

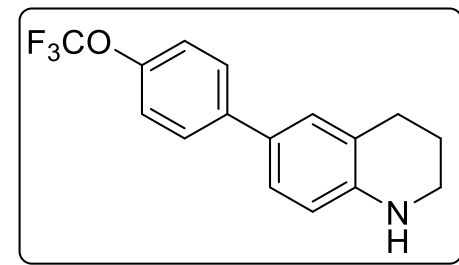

-57.8 ;

\section{2cl: 6-(4-(trifluoromethoxy)phenyl)-1,2,3,4-tetrahydroquinoline $\mathrm{e}^{[56]}$}

Yield: 95\%; ${ }^{1} \mathrm{H}$ NMR (400 MHz, Chloroform- $d$ ) $\delta 7.54-7.45(\mathrm{~m}, 2 \mathrm{H}), 7.23-7.13(\mathrm{~m}$, $4 \mathrm{H}), 6.54-6.46(\mathrm{~m}, 1 \mathrm{H}), 3.93(\mathrm{~s}, 1 \mathrm{H}), 3.36-3.23(\mathrm{~m}, 2 \mathrm{H}), 2.81(\mathrm{t}, J=6.4 \mathrm{~Hz}, 2 \mathrm{H}), 2.02$ $-1.85(\mathrm{~m}, 2 \mathrm{H}) ;{ }^{13} \mathrm{C}$ NMR (101 MHz, Chloroform- $d$ ) $\delta$ 147.6, 144.6, 140.4, 128.3, 128.2, $127.4,125.5,121.6,121.2,114.4,42.0,27.2,22.1 ;{ }^{19} \mathrm{~F}$ NMR (376 MHz, Chloroform- $d$ ) $\delta$

The obtained spectroscopic data were in agreement with the reported data for this compound 2cl;

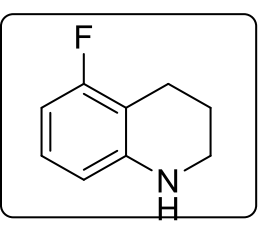

The obtained spectroscopic data were in agreement with the reported data for this compound $\mathbf{2 c m}$;

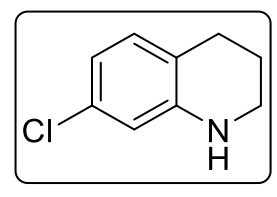

\section{2cn: 7-chloro-1,2,3,4-tetrahydroquinoline ${ }^{[62]}$}

Yield: 98\%, ${ }_{1}^{1} \mathrm{H}$ NMR (400 MHz, Chloroform- $d$ ) $\delta 6.83(\mathrm{dt}, J=7.9,1.0 \mathrm{~Hz}, 1 \mathrm{H}), 6.53(\mathrm{dd}, J=8.0$, $2.1 \mathrm{~Hz}, 1 \mathrm{H}), 6.43(\mathrm{~d}, J=2.1 \mathrm{~Hz}, 1 \mathrm{H}), 3.88(\mathrm{~s}, 1 \mathrm{H}), 3.33-3.24(\mathrm{~m}, 2 \mathrm{H}), 2.70(\mathrm{t}, J=6.4 \mathrm{~Hz}, 2 \mathrm{H})$, 1.91 (qdd, $J=6.3,4.2,2.5 \mathrm{~Hz}, 2 \mathrm{H}) ;{ }^{13} \mathrm{C}$ NMR (101 MHz, Chloroform- $d$ ) $\delta$ 145.7, 131.9, 130.4, 119.6, 116.5, 113.4, 41.7, 26.6, 21.8;

The obtained spectroscopic data were in agreement with the reported data for this compound $\mathbf{2} \mathbf{c n}$;

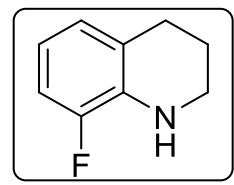

\section{2co: 8-fluoro-1,2,3,4-tetrahydroquinoline ${ }^{[63]}$}

Yield: 98\%; ${ }^{1} \mathrm{H}$ NMR (600 MHz, Chloroform- $d$ ) $\delta 6.79(\mathrm{dd}, J=11.3,8.1 \mathrm{~Hz}, 1 \mathrm{H}), 6.74(\mathrm{~d}, J=7.6 \mathrm{~Hz}$, $1 \mathrm{H}), 6.50(\mathrm{td}, J=7.8,5.4 \mathrm{~Hz}, 1 \mathrm{H}), 4.00(\mathrm{~s}, 1 \mathrm{H}), 3.34(\mathrm{t}, J=5.5 \mathrm{~Hz}, 2 \mathrm{H}), 2.78(\mathrm{t}, J=6.4 \mathrm{~Hz}, 2 \mathrm{H}), 1.96$ 
$(\mathrm{p}, J=6.1 \mathrm{~Hz}, 2 \mathrm{H}) ;{ }^{13} \mathrm{C} \mathrm{NMR}(151 \mathrm{MHz}$, Chloroform- $d) \delta 133.1,124.5\left(\mathrm{~d}, \mathrm{~J}_{\mathrm{C}-\mathrm{F}}=15.1 \mathrm{~Hz}\right), 123.6,115.6,115.5,112.2$, 112.1, 41.3, 26.5, 21.8;

The obtained spectroscopic data were in agreement with the reported data for this compound 2co;

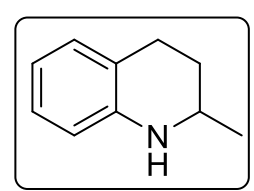

\section{2cp: 2-methyl-1,2,3,4-tetrahydroquinoline ${ }^{[54]}$}

Yield: 99\%; ${ }^{1} \mathrm{H}$ NMR (400 MHz, Chloroform- $d$ ) $\delta 7.03-6.95(\mathrm{~m}, 2 \mathrm{H}), 6.63(\mathrm{t}, J=7.4 \mathrm{~Hz}, 1 \mathrm{H}), 6.49$ $(\mathrm{d}, J=8.2 \mathrm{~Hz}, 1 \mathrm{H}), 3.71(\mathrm{~s}, 1 \mathrm{H}), 3.42(\mathrm{dqd}, J=9.3,6.2,2.8 \mathrm{~Hz}, 1 \mathrm{H}), 2.86(\mathrm{ddd}, J=16.9,11.5,5.7$ $\mathrm{Hz}, 1 \mathrm{H}), 2.75$ (ddd, $J=16.3,5.4,3.6 \mathrm{~Hz}, 1 \mathrm{H}), 1.95$ (ddt, $J=12.4,6.1,3.2 \mathrm{~Hz}, 1 \mathrm{H}), 1.61$ (tdd, $J=$ 12.2, 10.0, $5.3 \mathrm{~Hz}, 1 \mathrm{H}), 1.23$ (d, $J=6.3 \mathrm{~Hz}, 3 \mathrm{H}) ;{ }^{13} \mathrm{C}$ NMR (101 MHz, Chloroform-d) $\delta$ 144.8, 129.3, 126.7, 121.2, 117.0, 114.1, 47.2, 30.2, 26.6, 22.7;

The obtained spectroscopic data were in agreement with the reported data for this compound $\mathbf{2 c p}$.

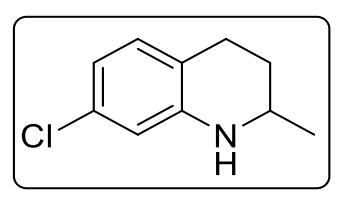

\section{2cq: 7-chloro-2-methyl-1,2,3,4-tetrahydroquinoline ${ }^{[56]}$}

Yield: 87\%; ${ }^{1} \mathrm{H}$ NMR (600 MHz, Chloroform- $d$ ) $\delta 6.85(\mathrm{~d}, J=7.9 \mathrm{~Hz}, 1 \mathrm{H}), 6.55(\mathrm{dd}, J=8.0$, $2.1 \mathrm{~Hz}, 1 \mathrm{H}), 6.44(\mathrm{~d}, J=2.1 \mathrm{~Hz}, 1 \mathrm{H}), 3.75(\mathrm{~s}, 1 \mathrm{H}), 3.39(\mathrm{dqd}, J=9.3,6.3,2.9 \mathrm{~Hz}, 1 \mathrm{H}), 2.76$ (ddd, $J=16.7,11.4,5.5 \mathrm{~Hz}, 1 \mathrm{H}), 2.69(\mathrm{dt}, J=16.3,4.5 \mathrm{~Hz}, 1 \mathrm{H}), 1.92$ (ddt, $J=12.7,5.4,3.4$ $\mathrm{Hz}, 1 \mathrm{H}), 1.55$ (dddd, $J=12.6,11.0,9.7,5.2 \mathrm{~Hz}, 1 \mathrm{H}), 1.21(\mathrm{~d}, J=6.3 \mathrm{~Hz}, 3 \mathrm{H}) ;{ }^{13} \mathrm{C}$ NMR $(151 \mathrm{MHz}, \mathrm{Chloroform}-d) \delta$ $145.7,131.9,130.2,119.3,116.6,113.3,47.0,29.8,26.1,22.5$;

The obtained spectroscopic data were in agreement with the reported data for this compound 2cq;

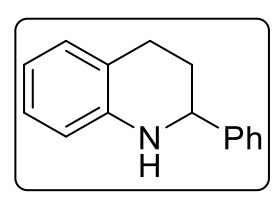

\section{2cr: 2-phenyl-1,2,3,4-tetrahydroquinoline ${ }^{[64]}$}

Yield: 90\%; ${ }^{1} \mathrm{H}$ NMR (400 MHz, Chloroform- $d$ ) $\delta 7.42-7.27(\mathrm{~m}, 4 \mathrm{H}), 7.31-7.22(\mathrm{~m}, 1 \mathrm{H}), 7.01(\mathrm{t}$, $J=7.3 \mathrm{~Hz}, 2 \mathrm{H}), 6.65$ (td, $J=7.4,1.2 \mathrm{~Hz}, 1 \mathrm{H}), 6.61-6.50(\mathrm{~m}, 1 \mathrm{H}), 4.43(\mathrm{dd}, J=9.3,3.3 \mathrm{~Hz}, 1 \mathrm{H})$, $4.03(\mathrm{~s}, 1 \mathrm{H}), 2.92(\mathrm{ddd}, J=16.3,10.6,5.5 \mathrm{~Hz}, 1 \mathrm{H}), 2.73(\mathrm{dt}, J=16.4,4.8 \mathrm{~Hz}, 1 \mathrm{H}), 2.12(\mathrm{dtd}, J=$ 13.2, 5.1, $3.4 \mathrm{~Hz}, 1 \mathrm{H}), 1.99$ (dddd, $J=12.9,10.6,9.3,5.1 \mathrm{~Hz}, 1 \mathrm{H}), 1.27(\mathrm{~d}, J=11.7 \mathrm{~Hz}, 4 \mathrm{H}), 0.92-0.81(\mathrm{~m}, 1 \mathrm{H}) . ;{ }^{13} \mathrm{C}$ NMR (101 MHz, Chloroform-d) $\delta$ 144.8, 144.8, 129.3, 128.6, 127.5, 126.9, 126.6, 120.9, 117.2, 114.0, 56.3, 31.0, 26.4; The obtained spectroscopic data were in agreement with the reported data for this compound $\mathbf{2 c r}$;

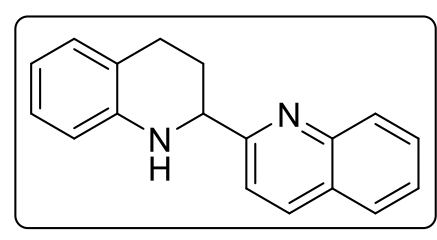

\section{2cs: 1,2,3,4-tetrahydro-2,2'-biquinoline ${ }^{[65]}$}

Yield: $85 \%$; ${ }^{1} \mathrm{H}$ NMR (400 MHz, Chloroform- $d$ ) $\delta 8.14(\mathrm{~d}, J=8.5 \mathrm{~Hz}, 2 \mathrm{H}), 8.09$ (dd, $J$ $=8.4,1.1 \mathrm{~Hz}, 2 \mathrm{H}), 7.81(\mathrm{dd}, J=8.1,1.4 \mathrm{~Hz}, 2 \mathrm{H}), 7.72(\mathrm{ddd}, J=8.5,6.9,1.5 \mathrm{~Hz}, 2 \mathrm{H})$, $7.59-7.48(\mathrm{~m}, 4 \mathrm{H}), 7.10-7.01(\mathrm{~m}, 3 \mathrm{H}), 7.01(\mathrm{~d}, J=1.6 \mathrm{~Hz}, 1 \mathrm{H}), 6.68(\mathrm{td}, J=7.8,7.2$, $1.4 \mathrm{~Hz}, 4 \mathrm{H}), 4.83(\mathrm{~s}, 2 \mathrm{H}), 4.75(\mathrm{dd}, J=9.6,3.5 \mathrm{~Hz}, 2 \mathrm{H}), 3.00(\mathrm{ddd}, J=16.1,10.7,5.2$ $\mathrm{Hz}, 2 \mathrm{H}), 2.78(\mathrm{dt}, J=16.2,4.7 \mathrm{~Hz}, 2 \mathrm{H}), 2.35(\mathrm{dtd}, J=13.1,5.0,3.4 \mathrm{~Hz}, 2 \mathrm{H}), 2.04$ (dddd, $J=12.8,10.7,9.5,4.8 \mathrm{~Hz}, 2 \mathrm{H})$, $1.27(\mathrm{~d}, J=11.5 \mathrm{~Hz}, 1 \mathrm{H}), 1.27(\mathrm{~s}, 1 \mathrm{H}), 0.92-0.81(\mathrm{~m}, 0 \mathrm{H}) ;{ }^{13} \mathrm{C}$ NMR $(101 \mathrm{MHz}$, Chloroform- $d) \delta 163.0,147.5,144.2$, 136.9, 129.7, 129.3, 129.1, 127.6, 127.5, 127.0, 126.3, 121.1, 118.9, 117.3, 114.6, 57.6, 29.1, 26.6;

The obtained spectroscopic data were in agreement with the reported data for this compound 2cs; 


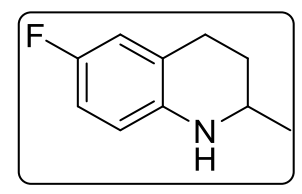

2ct: 6-fluoro-2-methyl-1,2,3,4-tetrahydroquinoline ${ }^{[65]}$

Yield: 96\%; ${ }^{1} \mathrm{H}$ NMR (600 MHz, Chloroform- $d$ ) $\delta 6.68(\mathrm{t}, J=8.3 \mathrm{~Hz}, 2 \mathrm{H}), 6.44-6.37(\mathrm{~m}, 1 \mathrm{H})$, $3.58(\mathrm{~s}, 1 \mathrm{H}), 3.35$ (ddd, $J=9.7,6.3,2.7 \mathrm{~Hz}, 1 \mathrm{H}), 2.83(\mathrm{ddd}, J=17.2,11.6,6.0 \mathrm{~Hz}, 1 \mathrm{H}), 2.70$ (ddd, $J=16.7,5.5,3.3 \mathrm{~Hz}, 1 \mathrm{H}), 1.92(\mathrm{ddt}, J=12.7,6.0,3.0 \mathrm{~Hz}, 1 \mathrm{H}), 1.62-1.50(\mathrm{~m}, 1 \mathrm{H}), 1.21(\mathrm{dd}, J=$ 6.3, $1.2 \mathrm{~Hz}, 3 \mathrm{H}) ;{ }^{19} \mathrm{~F}$ NMR (565 MHz, Chloroform- $d$ ) $\delta-128.33 ;{ }^{13} \mathrm{C}$ NMR (151 MHz, Chloroform- $d$ ) $\delta 156.3,154.7$, $141.0,122.5,122.5,115.5,115.3,114.8,114.7,113.2,113.1,47.3,29.9,26.7,22.5$;

The obtained spectroscopic data were in agreement with the reported data for this compound 2ct;

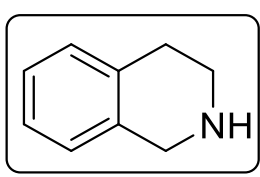

\section{2da: 1,2,3,4-tetrahydroisoquinoline ${ }^{[60]}$}

Yield: 89\%; ${ }^{1} \mathrm{H}$ NMR (600 MHz, Chloroform- $d$ ) $\delta 7.17-7.11(\mathrm{~m}, 2 \mathrm{H}), 7.11-7.05(\mathrm{~m}, 1 \mathrm{H}), 7.04-$ $6.97(\mathrm{~m}, 1 \mathrm{H}), 4.02(\mathrm{~s}, 2 \mathrm{H}), 3.14(\mathrm{td}, J=5.9,1.1 \mathrm{~Hz}, 2 \mathrm{H}), 2.80(\mathrm{t}, J=6.0 \mathrm{~Hz}, 2 \mathrm{H}), 1.80-1.64(\mathrm{~m}$, $1 \mathrm{H}) ;{ }^{13} \mathrm{C}$ NMR (151 MHz, Chloroform-d) $\delta$ 136.1, 134.8, 129.4, 126.2, 126.0, 125.7, 48.4, 44.0,

29.3;

The obtained spectroscopic data were in agreement with the reported data for this compound $\mathbf{2 d a}$.

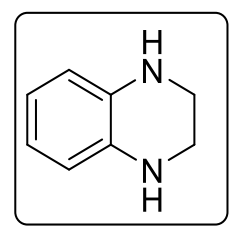

\section{2db: 1,2,3,4-tetrahydroquinoxaline ${ }^{[60]}$}

Yield: 98\%; ${ }^{1} \mathrm{H}$ NMR (400 MHz, Chloroform- $d$ ) $\delta 6.58(\mathrm{dd}, J=5.8,3.4 \mathrm{~Hz}, 2 \mathrm{H}), 6.49$ (dd, $J=5.8,3.4$ $\mathrm{Hz}, 2 \mathrm{H}), 3.64$ (brs, 2H), 3.41 (s, 4H); ${ }^{13} \mathrm{C}$ NMR (101 MHz, Chloroform- $d$ ) $\delta$ 133.7, 118.8, 114.7, 41.4; The obtained spectroscopic data were in agreement with the reported data for this compound $\mathbf{2} \mathbf{d b}$.

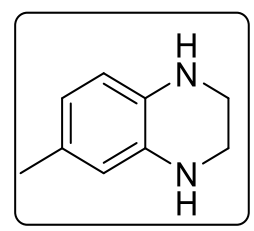

\section{2dc: 6-methyl-1,2,3,4-tetrahydroquinoxaline ${ }^{[66]}$}

Yield: 95\%; ${ }^{1} \mathrm{H}$ NMR (400 MHz, Chloroform- $d$ ) $\delta 6.46-6.37(\mathrm{~m}, 2 \mathrm{H}), 6.34(\mathrm{~d}, J=1.7 \mathrm{~Hz}, 1 \mathrm{H}), 3.45$ - $3.36(\mathrm{~m}, 5 \mathrm{H}), 2.18(\mathrm{~s}, 3 \mathrm{H}) ;{ }^{13} \mathrm{C}$ NMR (101 MHz, Chloroform-d) $\delta$ 133.8, 131.2, 128.4, 119.1, 115.5, 115.0, 41.6, 41.6, 20.7;

The obtained spectroscopic data were in agreement with the reported data for this compound $\mathbf{2 d c}$;

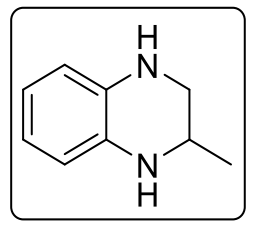

\section{2dd: 2-methyl-1,2,3,4-tetrahydroquinoxaline ${ }^{[67]}$}

Yield: 92\%; ${ }^{1} \mathrm{H}$ NMR (400 MHz, Chloroform- $d$ ) $\delta 6.66-6.58(\mathrm{~m}, 2 \mathrm{H}), 6.58-6.48(\mathrm{~m}, 2 \mathrm{H}), 3.63(\mathrm{~s}$, 2H), 3.54 (dqd, $J=8.1,6.3,2.9 \mathrm{~Hz}, 1 \mathrm{H}), 3.34$ (dd, $J=10.7,2.9 \mathrm{~Hz}, 1 \mathrm{H}), 3.07$ (dd, $J=10.7,8.2 \mathrm{~Hz}$, $1 \mathrm{H}), 1.31(\mathrm{~d}, J=11.9 \mathrm{~Hz}, 3 \mathrm{H}), 1.27(\mathrm{~s}, 1 \mathrm{H}), 1.22(\mathrm{~d}, J=6.3 \mathrm{~Hz}, 3 \mathrm{H}) ;{ }^{13} \mathrm{C}$ NMR $(101 \mathrm{MHz}$, Chloroform- $d$ ) $\delta 133.6,133.2,118.8,114.5,114.5,48.3,45.7,19.9$;

The obtained spectroscopic data were in agreement with the reported data for this compound 2dd;

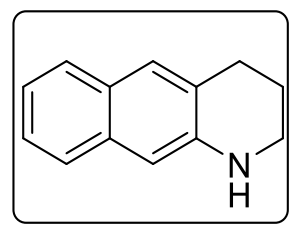

\section{2de: 1,2,3,4-tetrahydrobenzo[g]quinoline}

Yield: 98\%; ${ }^{1} \mathrm{H}$ NMR (400 MHz, Chloroform- $d$ ) $\delta 7.78-7.68(\mathrm{~m}, 1 \mathrm{H}), 7.71-7.63(\mathrm{~m}, 1 \mathrm{H}), 7.43$ $-7.32(\mathrm{~m}, 2 \mathrm{H}), 7.16(\mathrm{~d}, J=8.3 \mathrm{~Hz}, 1 \mathrm{H}), 7.10(\mathrm{~d}, J=8.4 \mathrm{~Hz}, 1 \mathrm{H}), 4.34(\mathrm{~s}, 1 \mathrm{H}), 3.50-3.42(\mathrm{~m}$, 2H), $2.90(\mathrm{t}, J=6.4 \mathrm{~Hz}, 2 \mathrm{H}), 2.07-1.97(\mathrm{~m}, 2 \mathrm{H}) ;{ }^{13} \mathrm{C}$ NMR (101 MHz, Chloroform- $d$ ) $\delta$ 139.0, 
133.1, 128.6, 128.6, 125.0, 124.8, 123.3, 119.5, 117.0, 115.8, 42.5, 27.5, 22.2; HR ESI-MS (positive ion): 184.1119 $\left([\mathrm{M}+\mathrm{H}]^{+}\right.$, calcd 184.1126) $\mathrm{m} / \mathrm{z}$;

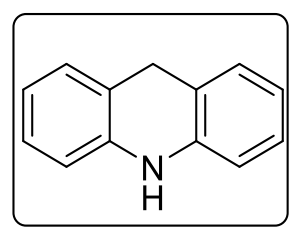

\section{2df: 9,10-dihydroacridine ${ }^{[68]}$}

Yield: 99\%; ${ }^{1} \mathrm{H}$ NMR (400 MHz, Chloroform- $d$ ) $\delta 7.15-7.05(\mathrm{~m}, 4 \mathrm{H}), 6.87$ (td, $J=7.4,1.2 \mathrm{~Hz}$, 2H), $6.68(\mathrm{dd}, J=7.9,1.1 \mathrm{~Hz}, 2 \mathrm{H}), 5.96(\mathrm{~s}, 1 \mathrm{H}), 4.07(\mathrm{~s}, 2 \mathrm{H}) ;{ }^{13} \mathrm{C}$ NMR $(101 \mathrm{MHz}$, Chloroform- $d)$ $\delta 140.2,128.6,127.0,120.7,120.1,113.5,31.4$;

The obtained spectroscopic data were in agreement with the reported data for this compound $\mathbf{2 d f}$;

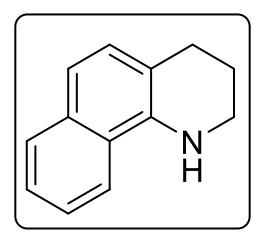

\section{2dg: 1,2,3,4-tetrahydrobenzo[h]quinoline ${ }^{[69]}$}

Yield: 99\%; ${ }^{1} \mathrm{H}$ NMR (600 MHz, Chloroform-d) $\delta 7.72(\mathrm{~d}, J=7.7 \mathrm{~Hz}, 1 \mathrm{H}), 7.66(\mathrm{~d}, J=7.7 \mathrm{~Hz}, 1 \mathrm{H})$, $7.41-7.33(\mathrm{~m}, 2 \mathrm{H}), 7.15(\mathrm{~d}, J=8.3 \mathrm{~Hz}, 1 \mathrm{H}), 7.10(\mathrm{~d}, J=8.2 \mathrm{~Hz}, 1 \mathrm{H}), 3.45(\mathrm{t}, J=5.5 \mathrm{~Hz}, 2 \mathrm{H}), 2.90$ $(\mathrm{t}, J=6.4 \mathrm{~Hz}, 2 \mathrm{H}), 2.07-1.94(\mathrm{~m}, 2 \mathrm{H}) ;{ }^{13} \mathrm{C}$ NMR (151 MHz, Chloroform-d) $\delta 139.1,133.1,128.6$, $128.6,124.9,124.7,123.3,119.5,116.9,115.8,42.5,25.4,22.2$. The obtained spectroscopic data were in agreement with the reported data for this compound $\mathbf{2 d g}$;

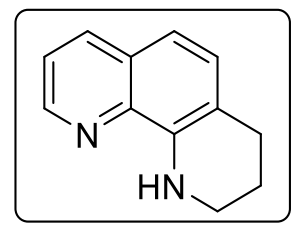

\section{2dh: 1,2,3,4-tetrahydro-1,10-phenanthroline ${ }^{[64]}$}

Yield: $85 \% ;{ }^{1} \mathrm{H}$ NMR (400 MHz, Chloroform- $d$ ) $\delta 8.67(\mathrm{dd}, J=4.2,1.7 \mathrm{~Hz}, 1 \mathrm{H}), 8.00(\mathrm{dd}, J=8.2$, $1.7 \mathrm{~Hz}, 1 \mathrm{H}), 7.28$ (dd, $J=8.3,4.2 \mathrm{~Hz}, 1 \mathrm{H}), 7.15$ (d, $J=8.2 \mathrm{~Hz}, 1 \mathrm{H}), 6.97$ (d, $J=8.3 \mathrm{~Hz}, 1 \mathrm{H}), 5.92$ $(\mathrm{s}, 1 \mathrm{H}), 3.52(\mathrm{t}, J=5.5 \mathrm{~Hz}, 2 \mathrm{H}), 2.92(\mathrm{t}, J=6.4 \mathrm{~Hz}, 2 \mathrm{H}), 2.12-1.99(\mathrm{~m}, 2 \mathrm{H}) ;{ }^{13} \mathrm{C} \mathrm{NMR}(101 \mathrm{MHz}$, Chloroform- $d$ ) $\delta 147.0,140.7,135.9,129.1,127.4,120.6,116.6,113.1,41.3,27.1,21.8$;

The obtained spectroscopic data were in agreement with the reported data for this compound $\mathbf{2 d h}$;

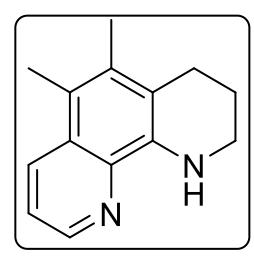

2di: 5,6-dimethyl-1,2,3,4-tetrahydro-1,10-phenanthroline ${ }^{[70]}$

Yield: 90\%; ${ }^{1} \mathrm{H}$ NMR (600 MHz, Chloroform- $d$ ) $\delta 8.63(\mathrm{~d}, J=4.1 \mathrm{~Hz}, 1 \mathrm{H}), 8.23(\mathrm{~d}, J=8.6 \mathrm{~Hz}, 1 \mathrm{H})$, $7.30(\mathrm{dd}, J=8.6,4.1 \mathrm{~Hz}, 1 \mathrm{H}), 5.93(\mathrm{~s}, 1 \mathrm{H}), 3.44$ (t, $J=5.5 \mathrm{~Hz}, 2 \mathrm{H}), 2.83$ (t, $J=6.5 \mathrm{~Hz}, 2 \mathrm{H}), 2.47$ (s, 3H), 2.31 (s, 3H), $2.15-2.02(\mathrm{~m}, 2 \mathrm{H}) ;{ }^{13} \mathrm{C}$ NMR (151 MHz, Chloroform-d) $\delta 145.7,139.0,136.8$, $134.2,132.2,126.2,120.3,117.0,116.8,40.8,25.2,22.7,15.9,13.7$;

The obtained spectroscopic data were in agreement with the reported data for this compound $2 \mathbf{d i}$.

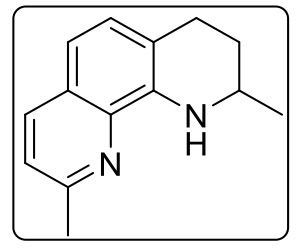

\section{2dj: 2,9-dimethyl-1,2,3,4-tetrahydro-1,10-phenanthroline ${ }^{[71]}$}

Yield: 85\%; ${ }^{1} \mathrm{H}$ NMR $\quad(600 \mathrm{MHz}$, Chloroform- $d) \delta 7.89(\mathrm{~d}, J=8.3 \mathrm{~Hz}, 1 \mathrm{H}), 7.17(\mathrm{~d}, J=8.2 \mathrm{~Hz}$, $1 \mathrm{H}), 7.10$ (d, $J=8.1 \mathrm{~Hz}, 1 \mathrm{H}), 6.95$ (d, $J=8.1 \mathrm{~Hz}, 1 \mathrm{H}), 5.86(\mathrm{~s}, 1 \mathrm{H}), 3.59$ (ddq, $J=12.8,6.5,4.1$,

$2.7 \mathrm{~Hz}, 1 \mathrm{H}), 3.00(\mathrm{ddd}, J=16.7,11.0,5.8 \mathrm{~Hz}, 1 \mathrm{H}), 2.87$ (dt, $J=16.6,4.5 \mathrm{~Hz}, 1 \mathrm{H}), 2.69$ (s, 3H), $2.05(\mathrm{ddt}, J=12.7,6.4,3.3 \mathrm{~Hz}, 1 \mathrm{H}), 1.82-1.56(\mathrm{~m}, 2 \mathrm{H}), 1.38(\mathrm{~d}, J=6.3 \mathrm{~Hz}, 3 \mathrm{H}) ;{ }^{13} \mathrm{C}$ NMR $(151 \mathrm{MHz}$, Chloroform-d) $\delta$ 
The obtained spectroscopic data were in agreement with the reported data for this compound $\mathbf{2 d j}$;

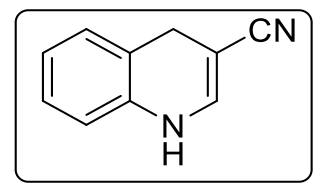

\section{2ea: 1,4-dihydroquinoline-3-carbonitrile}

White solid; Yield: 95\%; ${ }^{1} \mathrm{H}$ NMR (400 MHz, Chloroform- $d$ ) $\delta 7.08$ (td, $J=7.4,2.0 \mathrm{~Hz}, 1 \mathrm{H}$ ), $7.00-6.88(\mathrm{~m}, 2 \mathrm{H}), 6.82(\mathrm{~d}, J=5.9 \mathrm{~Hz}, 1 \mathrm{H}), 6.59(\mathrm{~d}, J=7.9 \mathrm{~Hz}, 1 \mathrm{H}), 6.27(\mathrm{~s}, 1 \mathrm{H}), 3.72(\mathrm{~s}, 2 \mathrm{H})$;

${ }^{13} \mathrm{C}$ NMR (101 MHz, Chloroform-d) $\delta$ 139.5, 135.7, 129.4, 127.7, 123.8, 121.3, 118.5, 115.2, 26.9; HR ESI-MS (positive ion): $157.0760\left([\mathrm{M}+\mathrm{H}]^{+}\right.$, calcd 157.0766) $\mathrm{m} / \mathrm{z}$.

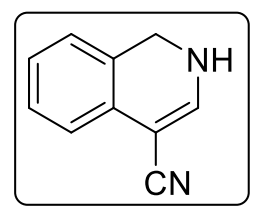

\section{2eb: 1,2-dihydroisoquinoline-4-carbonitrile}

White solid; Yield: $92 \% ;{ }^{1} \mathrm{H}$ NMR $(\mathbf{s} / \mathbf{c}=\mathbf{2 0})(600 \mathrm{MHz}$, Chloroform- $d) \delta 7.27-7.15(\mathrm{~m}, 2 \mathrm{H}), 7.10$ (t, $J=7.5 \mathrm{~Hz}, 1 \mathrm{H}), 7.03(\mathrm{~d}, J=6.7 \mathrm{~Hz}, 1 \mathrm{H}), 6.90(\mathrm{~d}, J=7.7 \mathrm{~Hz}, 1 \mathrm{H}), 4.79(\mathrm{~s}, 1 \mathrm{H}), 4.66(\mathrm{~s}, 2 \mathrm{H}) ;{ }^{13} \mathrm{C}$ NMR (151 MHz, Chloroform- $d$ ) $\delta$ 145.5, 128.8, 128.3, 126.7, 125.6, 124.6, 121.9, 45.0; HR ESI-MS (positive ion): $157.0758\left([\mathrm{M}+\mathrm{H}]^{+}\right.$, calcd 157.0766$) \mathrm{m} / \mathrm{z}$.

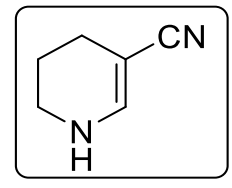

\section{2ec: 1,4,5,6-tetrahydropyridine-3-carbonitrile ${ }^{[72]}$}

Yield: 95\%; ${ }^{1} \mathrm{H}$ NMR (400 MHz, Chloroform- $d$ ) $\delta 6.90(\mathrm{~d}, J=6.0 \mathrm{~Hz}, 1 \mathrm{H}), 3.21(\mathrm{dq}, J=6.1,3.0 \mathrm{~Hz}$, $2 \mathrm{H}), 2.26(\mathrm{t}, J=6.2 \mathrm{~Hz}, 2 \mathrm{H}), 1.84(\mathrm{p}, J=6.0 \mathrm{~Hz}, 2 \mathrm{H}) .{ }^{13} \mathrm{C}$ NMR $(101 \mathrm{MHz}$, Chloroform- $d$ ) $\delta 144.3$,

123.1, 74.4, 40.2, 22.4, 20.5;

The obtained spectroscopic data were in agreement with the reported data for this compound 2ec;

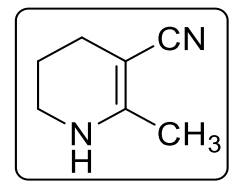

2ed: 2-methyl-1,4,5,6-tetrahydropyridine-3-carbonitrile ${ }^{[73]}$

Yield: 96\%; ${ }^{1} \mathrm{H}$ NMR (400 MHz, Chloroform- $d$ ) $\delta 4.31(\mathrm{~s}, 1 \mathrm{H}), 3.26-3.18(\mathrm{~m}, 2 \mathrm{H}), 2.29-2.21(\mathrm{~m}$, $2 \mathrm{H}), 2.01(\mathrm{t}, J=1.3 \mathrm{~Hz}, 3 \mathrm{H}), 1.84-1.73(\mathrm{~m}, 2 \mathrm{H}) ;{ }^{13} \mathrm{C}$ NMR $(101 \mathrm{MHz}$, Chloroform- $d$ ) $\delta$ 152.9, 123.6, $71.6,41.2,23.2,20.7,19.8$;

The obtained spectroscopic data were in agreement with the reported data for this compound 2ed.

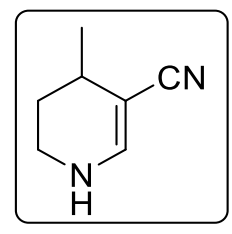

\section{2ee: 4-methyl-1,4,5,6-tetrahydropyridine-3-carbonitrile}

Yellow oil; Yield: 92\%; ${ }^{1} \mathrm{H}$ NMR (400 MHz, Chloroform- $d$ ) $\delta 6.89$ (dd, $\left.J=6.1,1.2 \mathrm{~Hz}, 1 \mathrm{H}\right), 4.45(\mathrm{~s}$, $1 \mathrm{H}), 3.30-3.15$ (m, 2H), 2.46 (tdd, $J=6.8,5.2,1.3 \mathrm{~Hz}, 1 \mathrm{H}), 1.94-1.82(\mathrm{~m}, 1 \mathrm{H}), 1.52$ (dtd, $J=13.5$, 6.8, $4.9 \mathrm{~Hz}, 1 \mathrm{H}), 1.17(\mathrm{~d}, J=6.9 \mathrm{~Hz}, 3 \mathrm{H}) ;{ }^{13} \mathrm{C}$ NMR $(101 \mathrm{MHz}$, Chloroform- $d$ ) $\delta 144.0,122.7,80.8$, 38.5, 28.8, 27.0, 20.8; HR ESI-MS (positive ion): 123.0918 ([M+H] $]^{+}$, calcd 123.0922) m/z. 


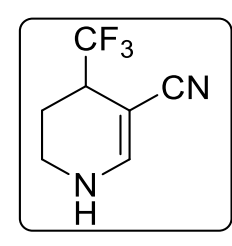

2ef: 4-(trifluoromethyl)-1,4,5,6-tetrahydropyridine-3-carbonitrile

Yellow oil; Yield: 89\%; ${ }^{1} \mathrm{H}$ NMR (600 MHz, Chloroform- $\left.d\right) \delta 6.99(\mathrm{~d}, J=6.6 \mathrm{~Hz}, 1 \mathrm{H}), 5.59(\mathrm{~d}, J=$ $2.0 \mathrm{~Hz}, 1 \mathrm{H}), 4.77(\mathrm{~s}, 1 \mathrm{H}), 4.34(\mathrm{dt}, J=4.5,2.1 \mathrm{~Hz}, 2 \mathrm{H}), 1.31(\mathrm{~d}, J=29.2 \mathrm{~Hz}, 1 \mathrm{H}) ;{ }^{19} \mathrm{~F}$ NMR $(565$

MHz, Chloroform- $d$ ) $\delta-67.7 ;{ }^{13} \mathrm{C}$ NMR (151 MHz, Chloroform- $d$ ) $\delta 148.7,122.3,120.5,118.1,113.4$, 113.3, 73.3, 41.8, 31.4, 30.2; HR ESI-MS (negative ion): 175.0482 ([M-H]', calcd 175.0483) m/z. 


\section{NMR Spectra}

L1; ${ }^{1} \mathrm{H}$ NMR; $\mathrm{CDCl}_{3} ; 400 \mathrm{MHz}$

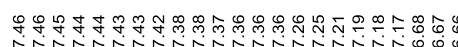

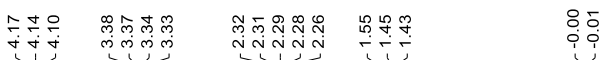
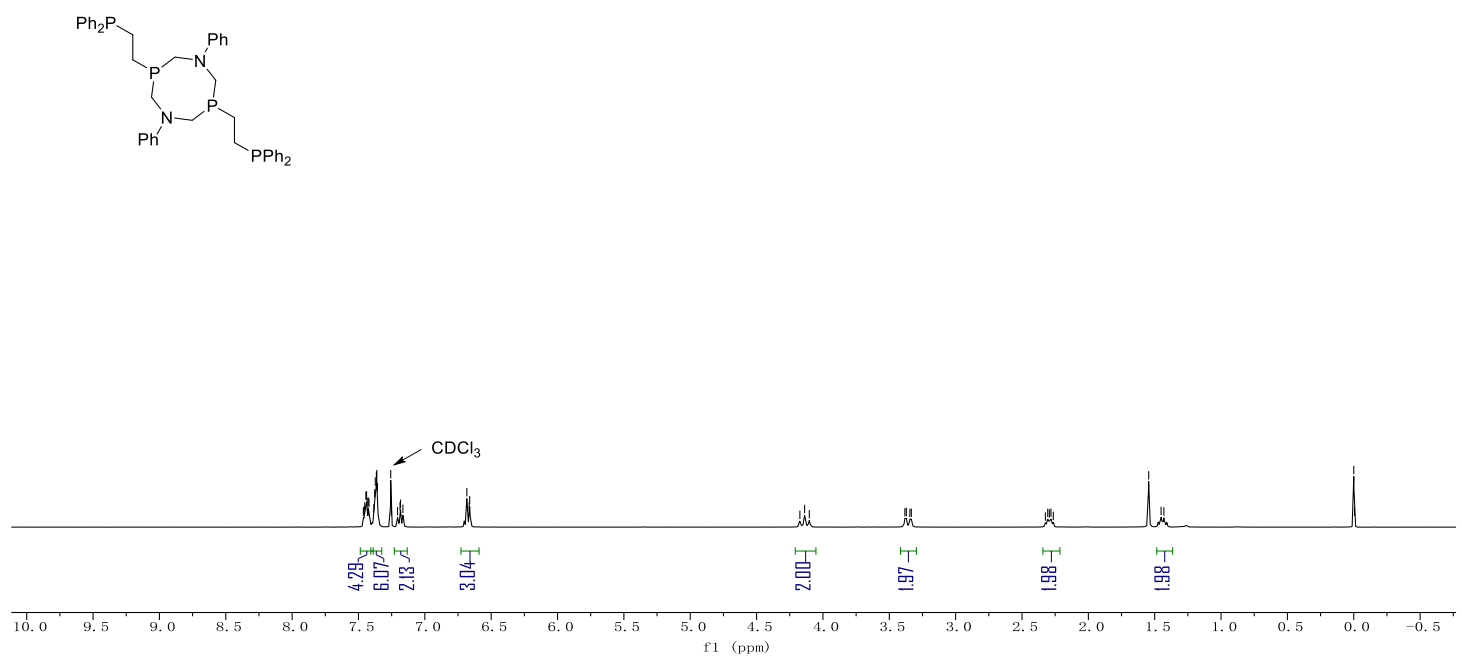

L1; ${ }^{31}$ P NMR; $\mathrm{CDCl}_{3}, 162 \mathrm{MHz}$
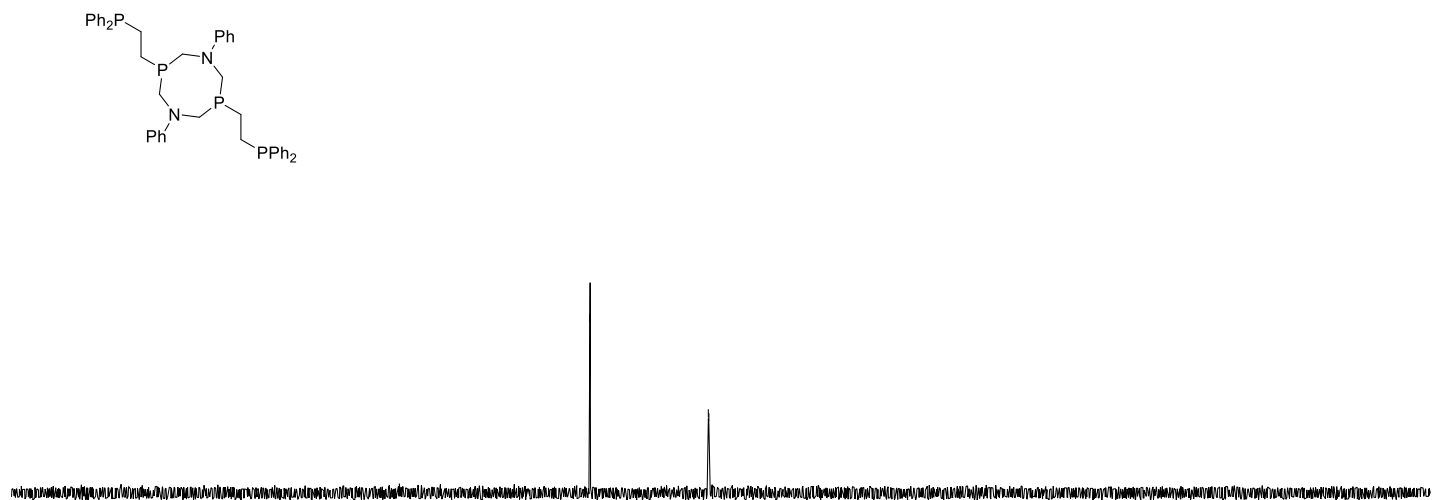

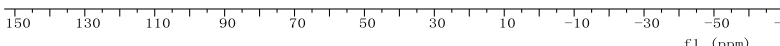




\section{Complex 3; ${ }^{1} \mathrm{H}$ NMR; $\mathrm{CD}_{3} \mathrm{CN} ; 400 \mathrm{MHz}$}

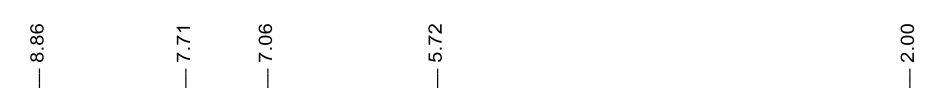
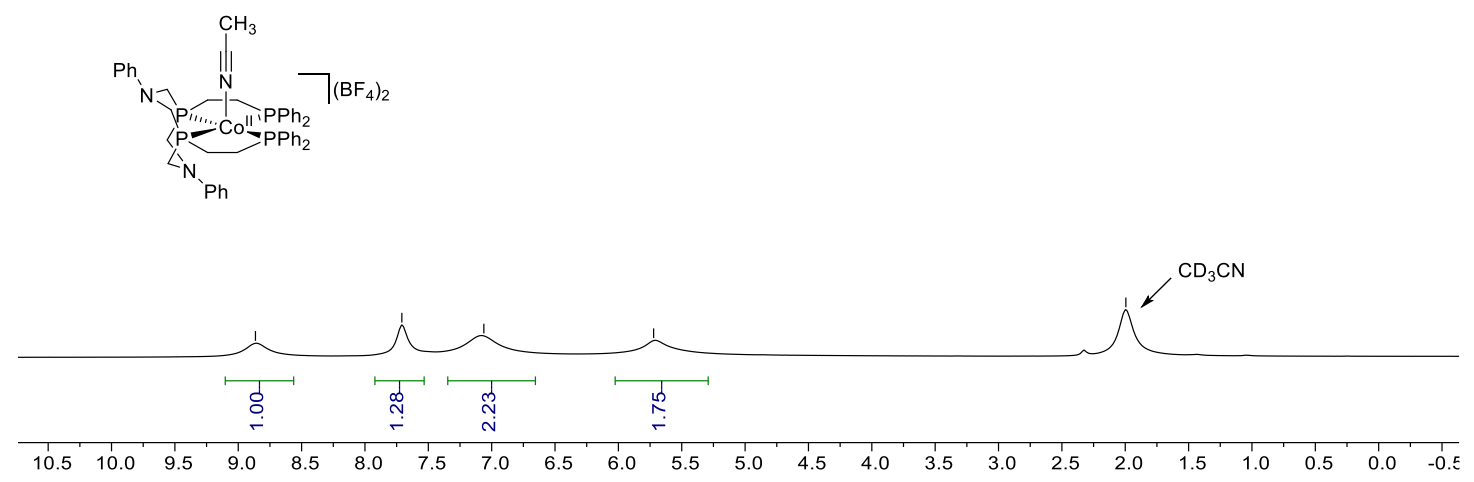

Complex 4; ${ }^{1} \mathrm{H}$ NMR; $\mathrm{d}_{8}$-THF; $400 \mathrm{MHz}$

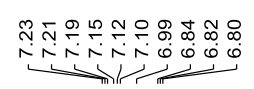

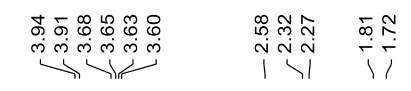

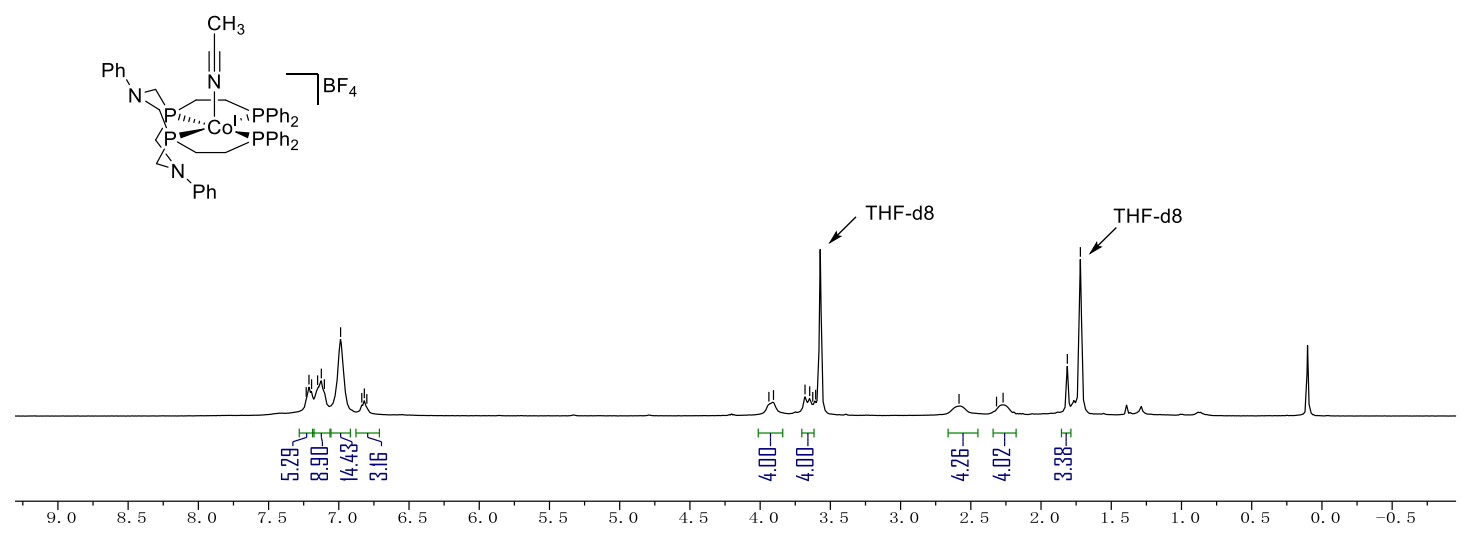


Complex 4; ${ }^{31} \mathrm{P}$ NMR; $\mathrm{d}_{8}$-THF; $162 \mathrm{MHz}$

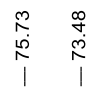

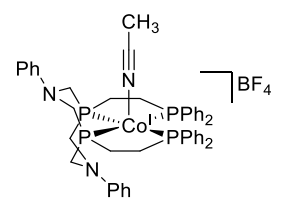

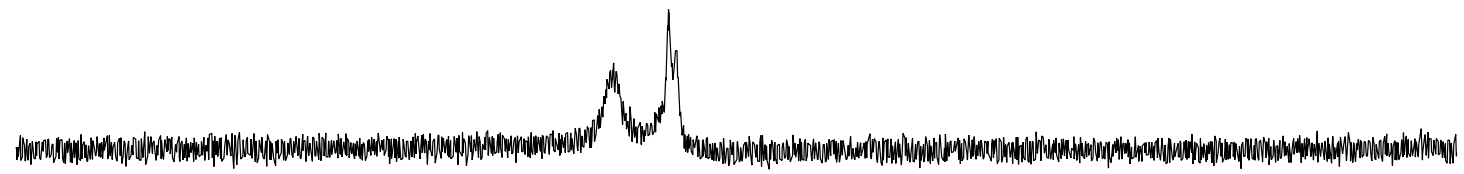

Complex 4; ${ }^{19} \mathrm{~F}$ NMR; $\mathrm{d}_{8}$-THF; $376 \mathrm{MHz}$

$$
\underset{\substack{i \\ \text { i }}}{\stackrel{\circ}{i}}
$$

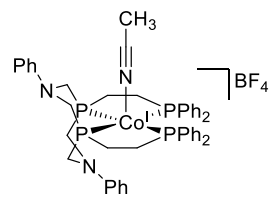


Complex 5; ${ }^{1} \mathrm{H}$ NMR; $\mathrm{d}_{8}$-THF, $400 \mathrm{MHz}$

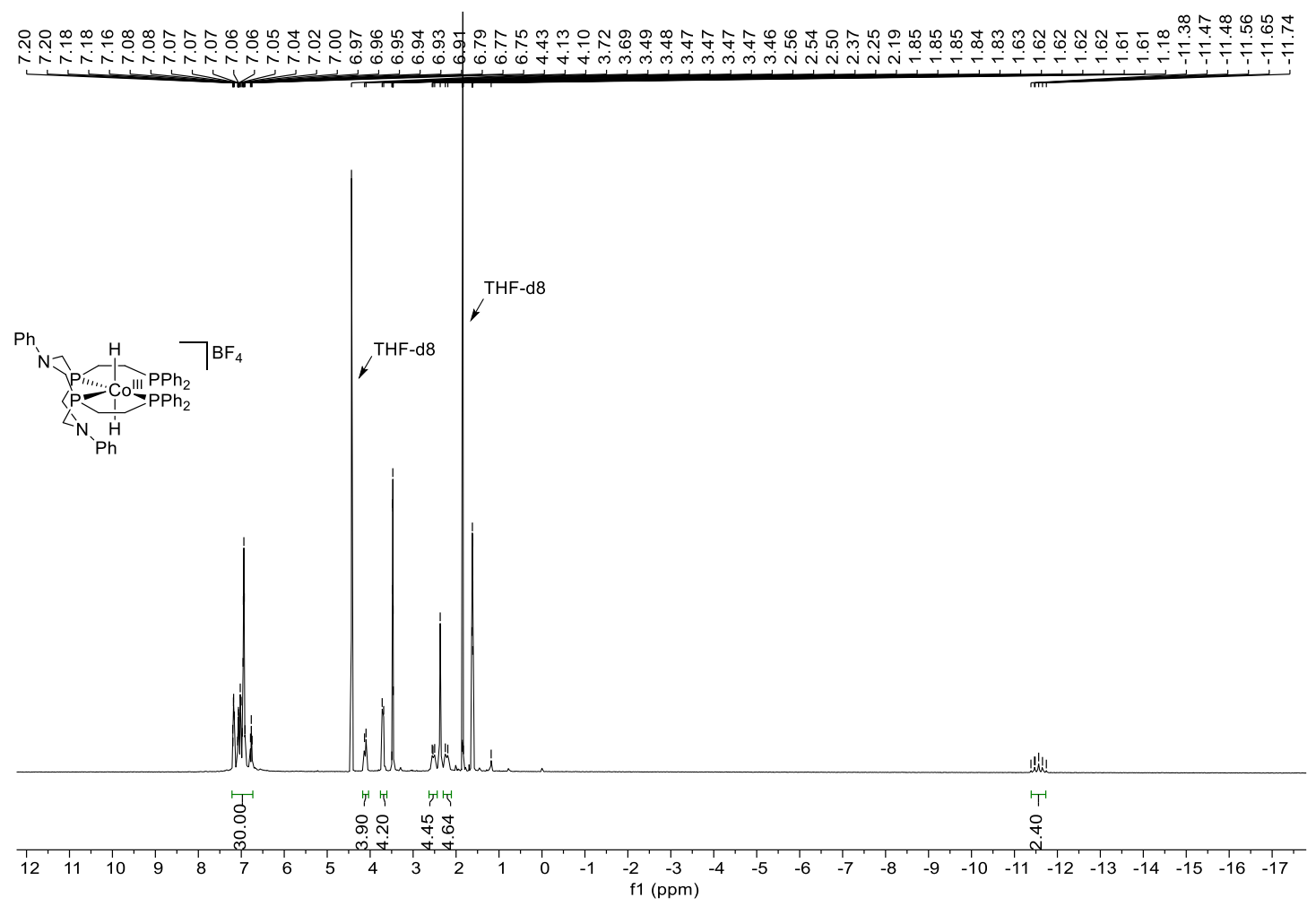

Complex 5; ${ }^{31} \mathrm{P}$ NMR; $\mathrm{d}_{8}$-THF; $162 \mathrm{MHz}$

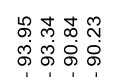

4

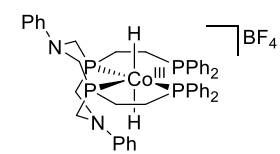

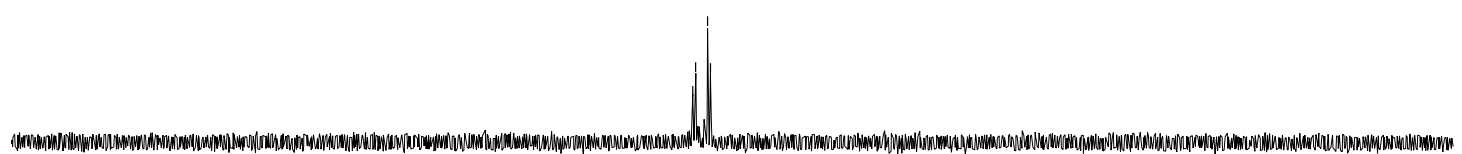

$\begin{array}{llllllllllllllllllllllllllllllllllll}230 & 220 & 210 & 200 & 190 & 180 & 170 & 160 & 150 & 140 & 130 & 120 & 110 & 100 & 90 & 80 & 70 & 60 & 50 & 40 & 30 & 20 & 10 & 0 & -10 & -20 & -30 & -40 & -50 & -60\end{array}$ $\mathrm{f} 1(\mathrm{ppm})$

NMR; $\mathrm{d}_{8}$-THF; $376 \mathrm{MHz}$ 

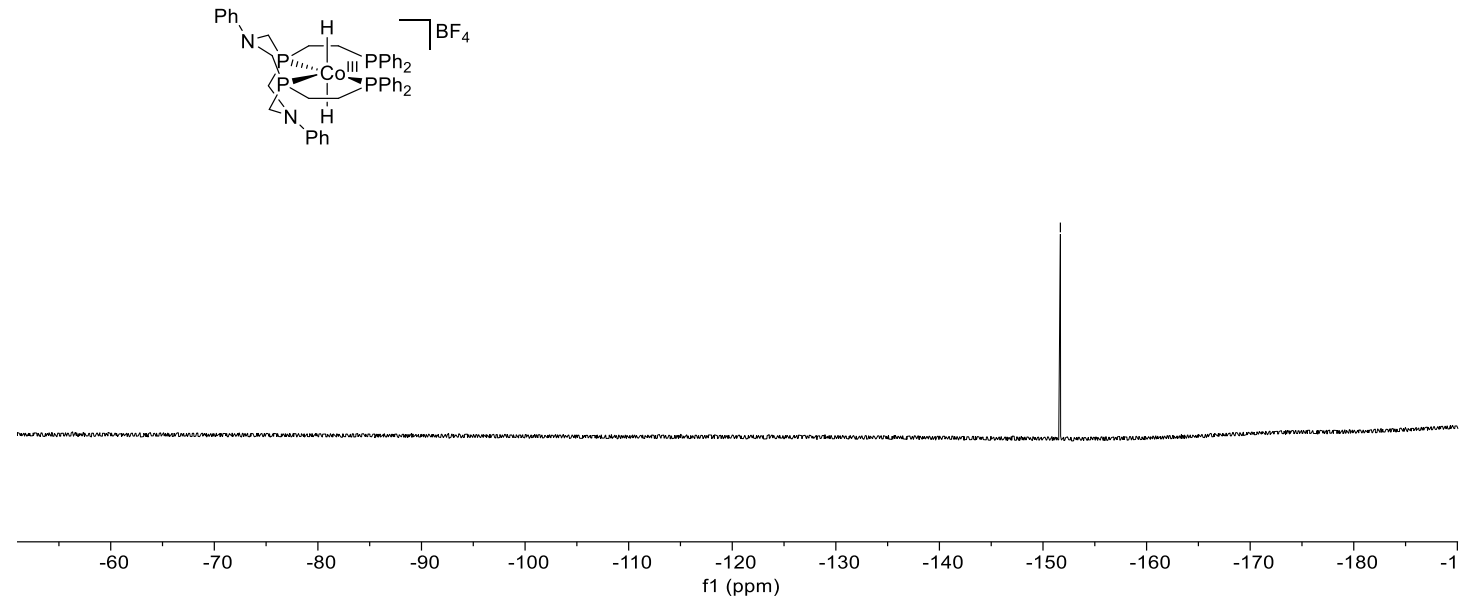

Complex 5; ${ }^{13} \mathrm{C}$ NMR; $\mathrm{d}_{8}$-THF; $101 \mathrm{MHz}$

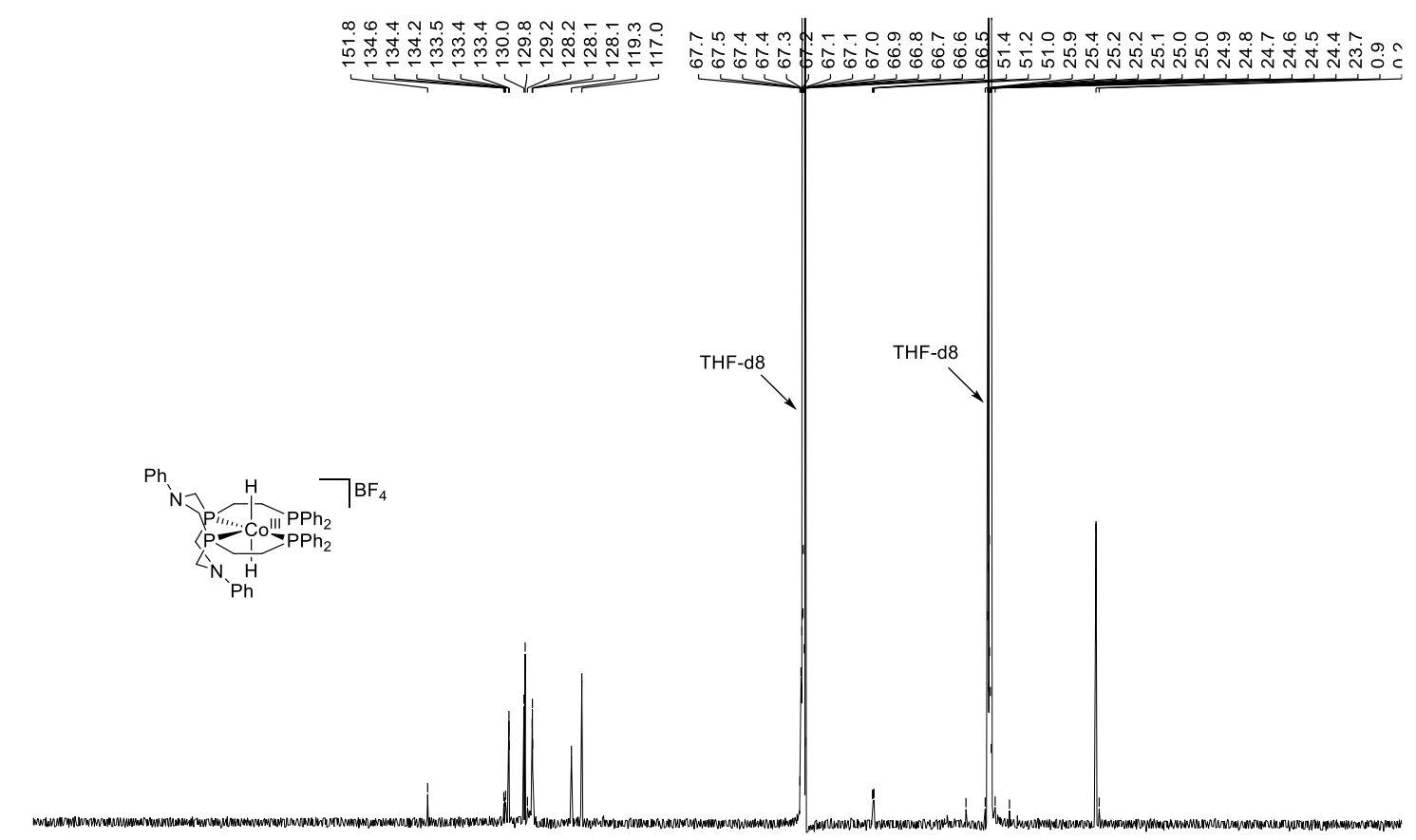

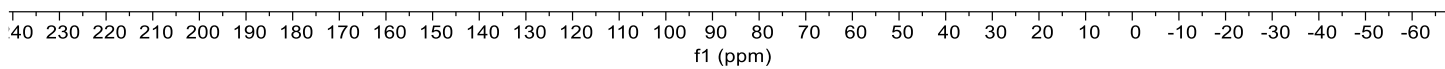

Complex 6; ${ }^{1} \mathrm{H} \mathrm{NMR} ; \mathrm{CD}_{3} \mathrm{CN} ; 400 \mathrm{MHz}$ 


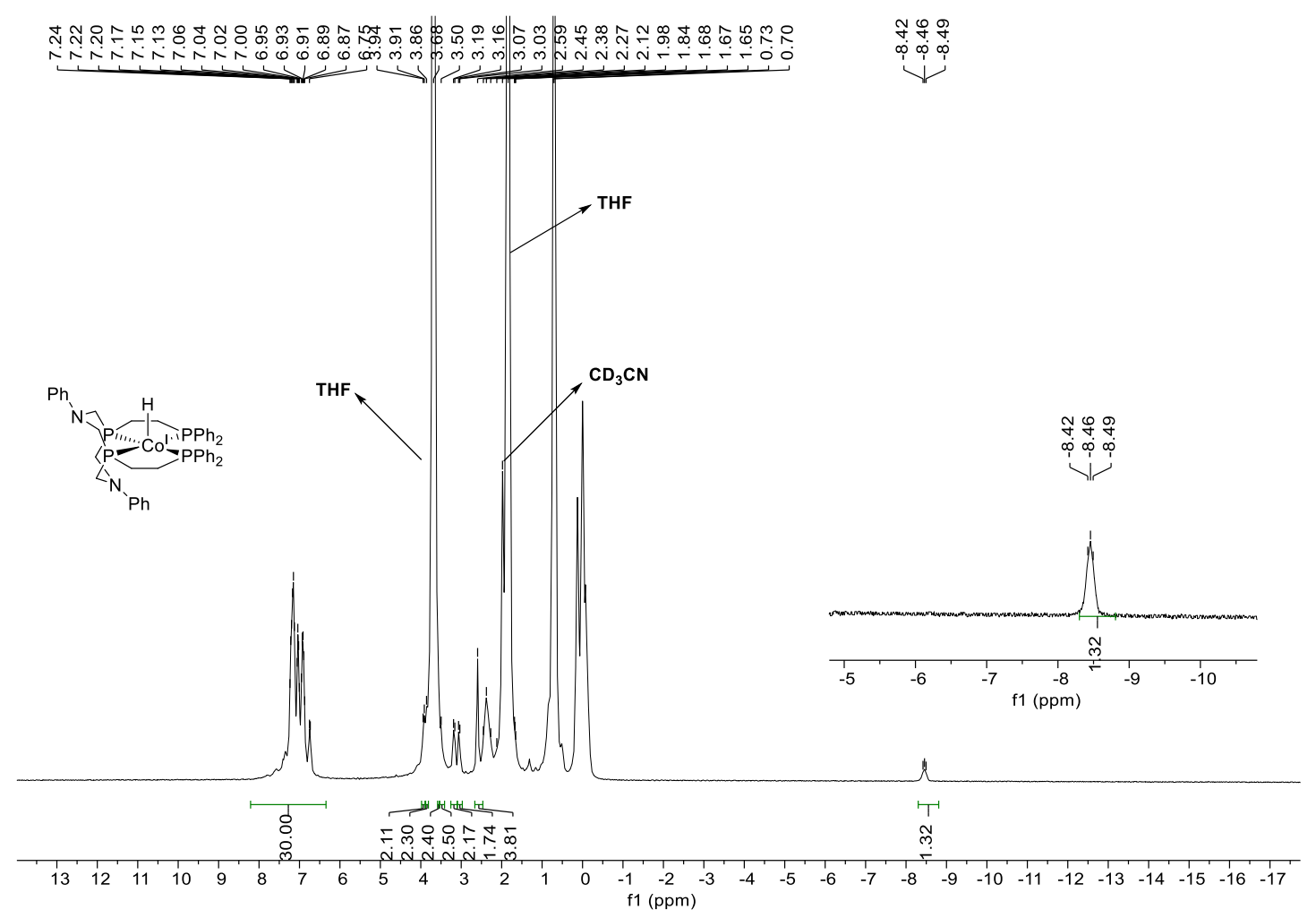

Complex 6; ${ }^{31} \mathrm{P}$ NMR; $\mathrm{CD}_{3} \mathrm{CN} ; 162 \mathrm{MHz}$

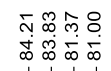

4

$\underbrace{\mathrm{Ph}}_{\mathrm{N}_{\mathrm{Ph}}}$

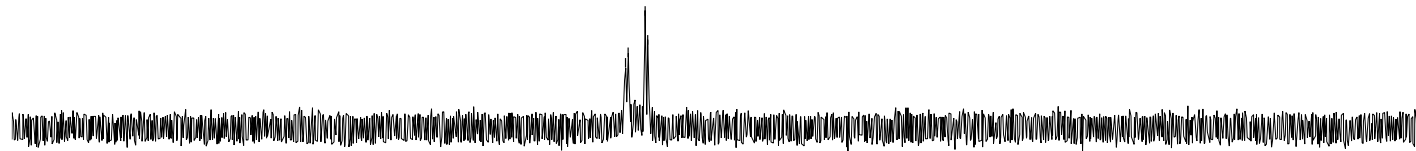

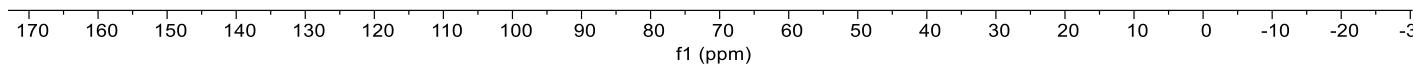

L2; ${ }^{1} \mathrm{H}$ NMR; $\mathrm{CDCl}_{3} ; 400 \mathrm{MHz}$ 


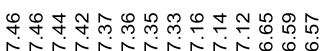

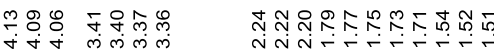

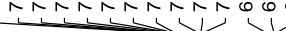

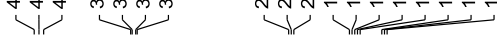
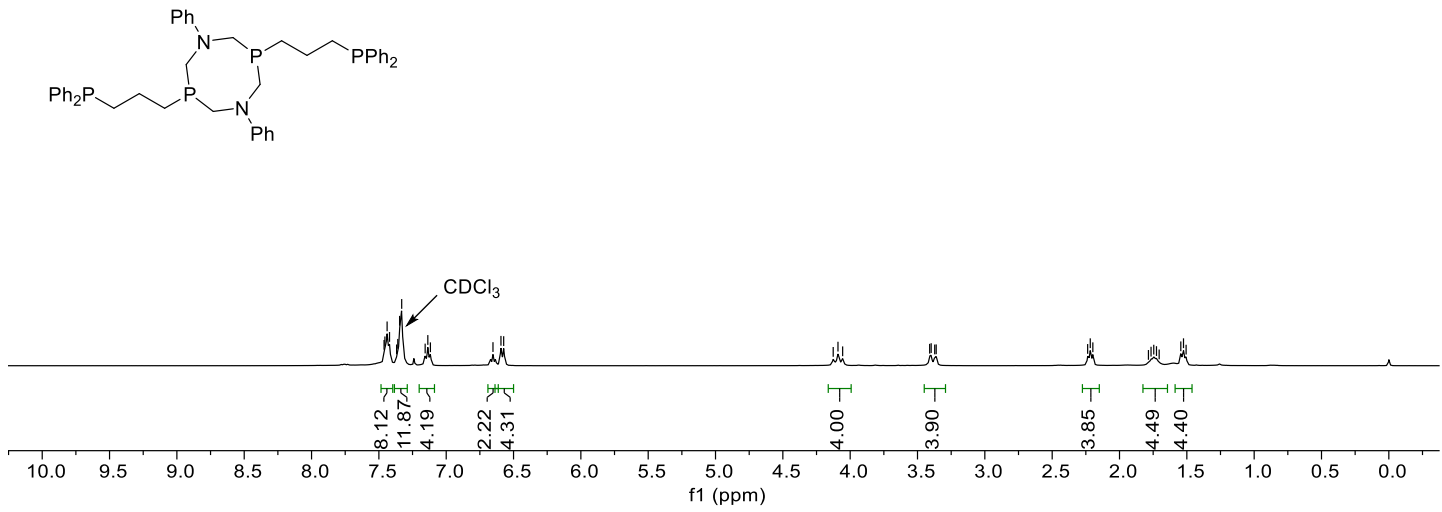

L2; ${ }^{31} \mathrm{P}$ NMR; $\mathrm{CDCl}_{3} ; 162 \mathrm{MHz}$

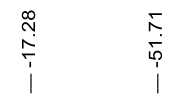<smiles>Pc1ccccc1</smiles>

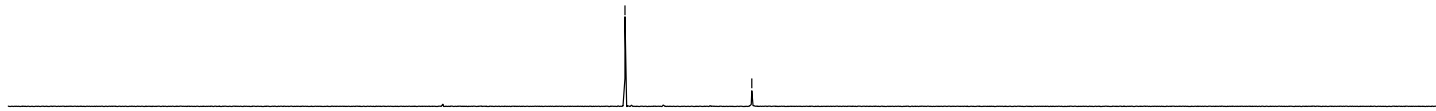

$\begin{array}{llll}140 & 120 & 100 & 100\end{array}$ -40
$\mathrm{f} 1(\mathrm{ppm})$ 
L2; ${ }^{13} \mathrm{C} \mathrm{NMR} ; \mathrm{CDCl}_{3} ; 101 \mathrm{MHz}$

\begin{tabular}{|c|c|}
\hline 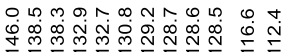 & \\
\hline
\end{tabular}

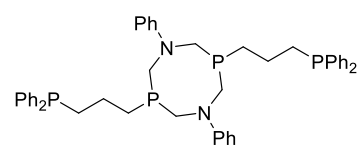
$\mathrm{CDCl}_{3}$

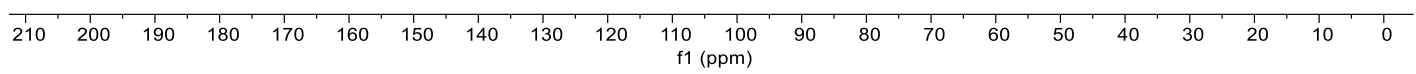

L3; ${ }^{1} \mathrm{H} \mathrm{NMR} ; \mathrm{CDCl}_{3} ; 400 \mathrm{MHz}$

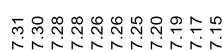

若

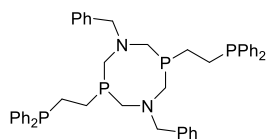

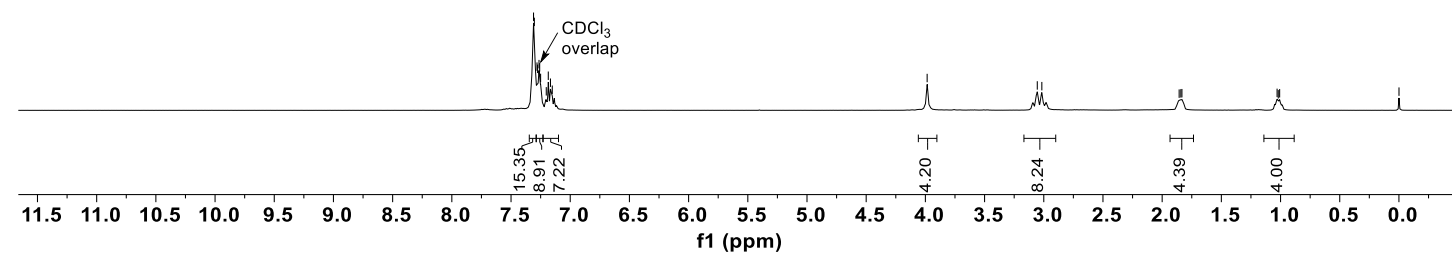


L3; ${ }^{31} \mathrm{P}$ NMR; $\mathrm{CDCl}_{3} ; 162 \mathrm{MHz}$

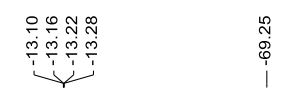
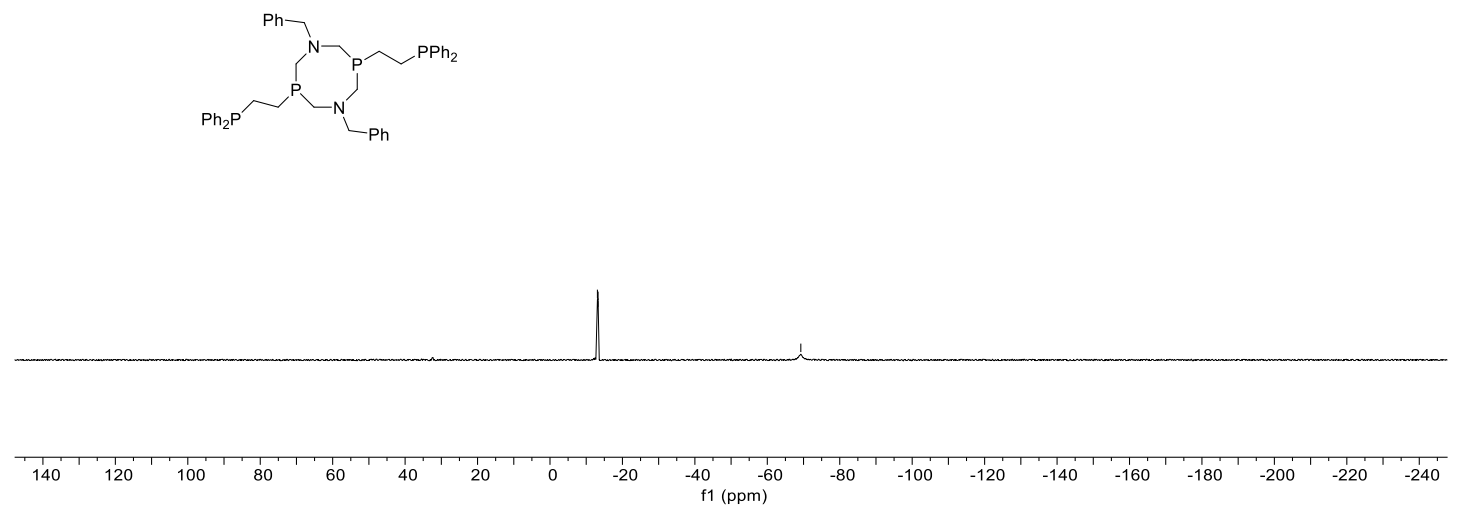

L3; ${ }^{13} \mathrm{C}$ NMR; $\mathrm{CDCl}_{3} ; 101 \mathrm{MHz}$
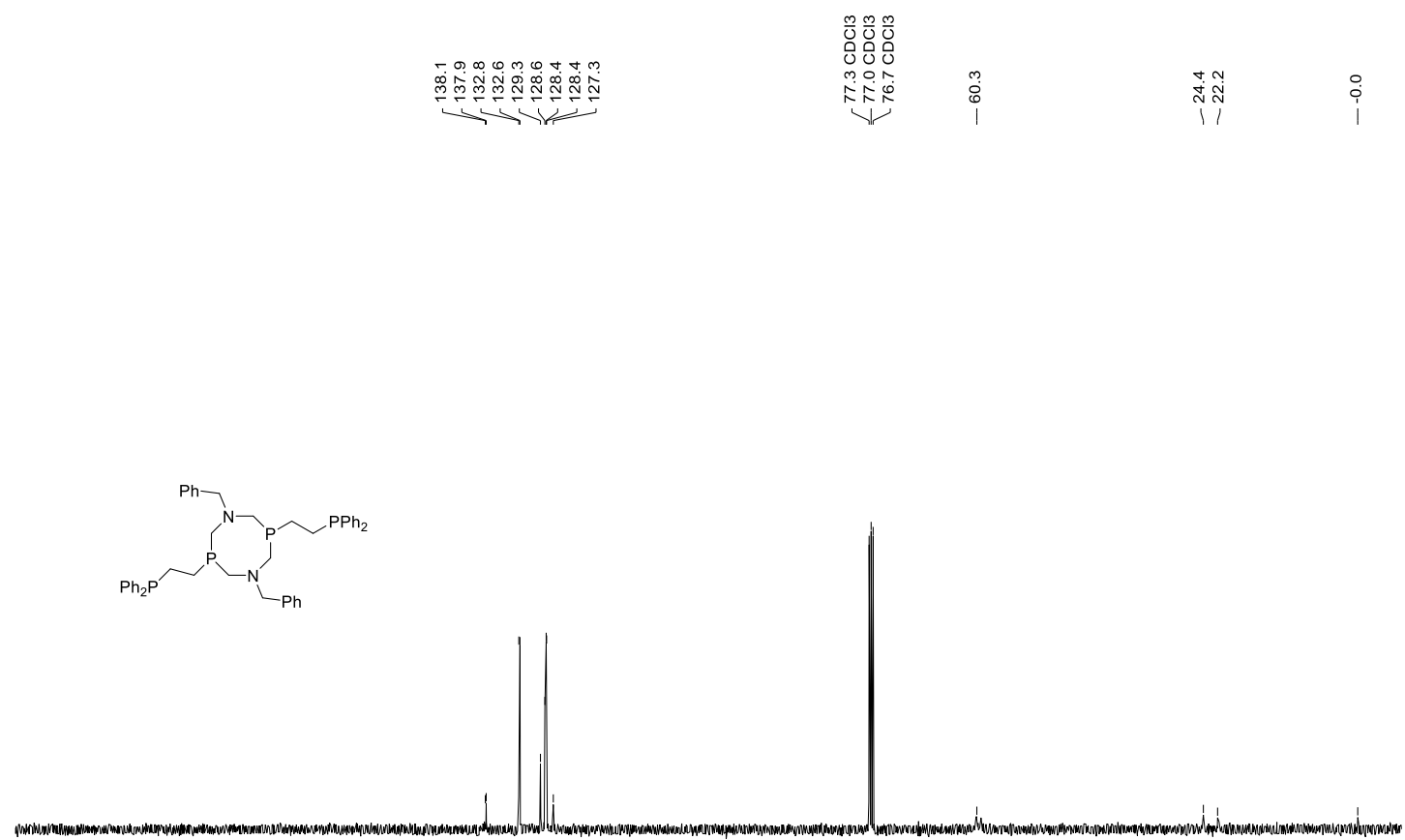

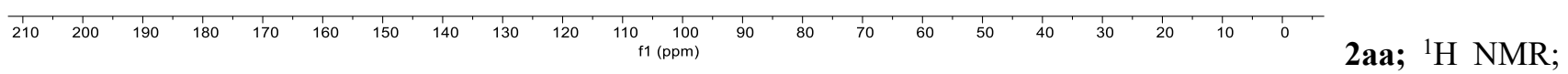



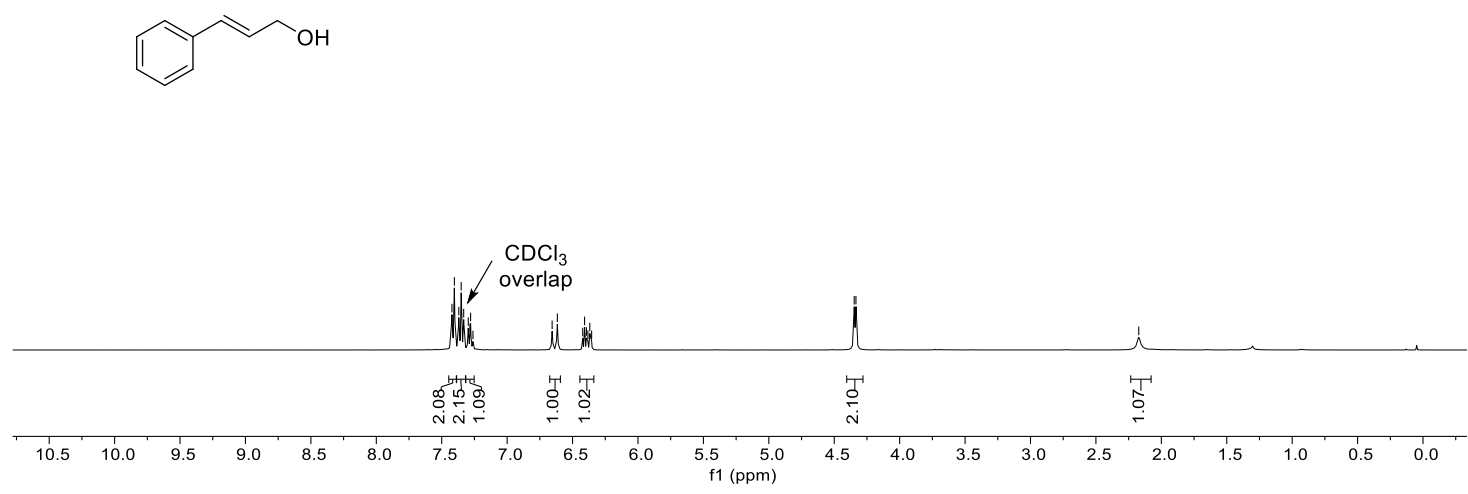

2aa; ${ }^{13} \mathrm{C} \mathrm{NMR} ; \mathrm{CDCl}_{3} ; 101 \mathrm{MHz}$

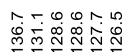

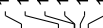

$\frac{m}{0} \frac{m}{0}$
0
000
0

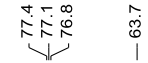
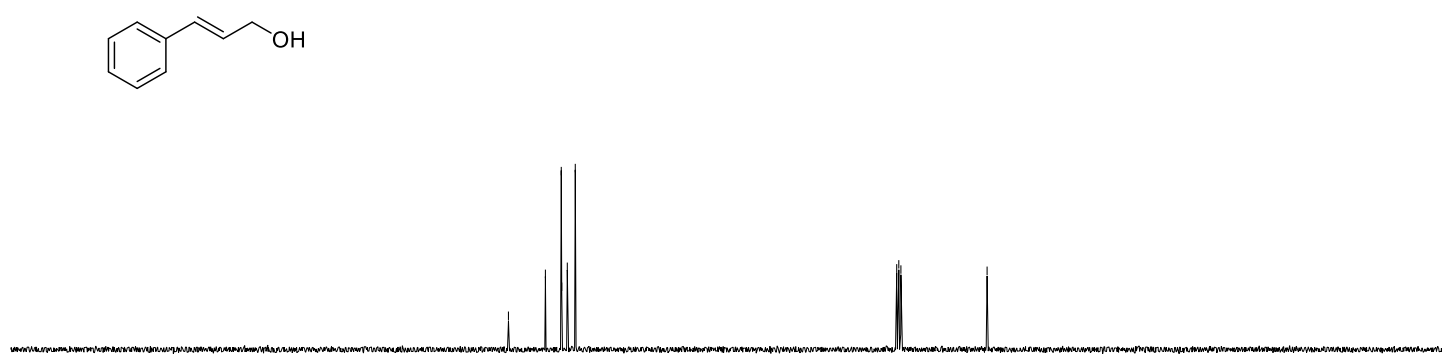

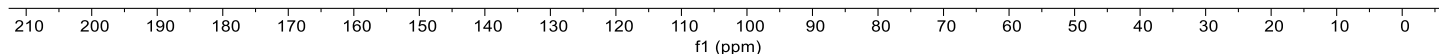

2ab; ${ }^{1} \mathrm{H} \mathrm{NMR} ; \mathrm{CDCl}_{3}, 400 \mathrm{MHz}$ 


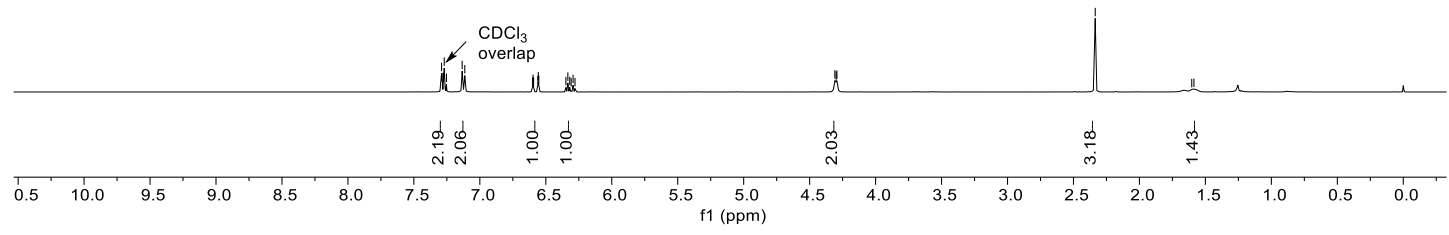

2ab; ${ }^{13} \mathrm{C} \mathrm{NMR} ; \mathrm{CDCl}_{3}, 101 \mathrm{MHz}$

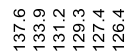
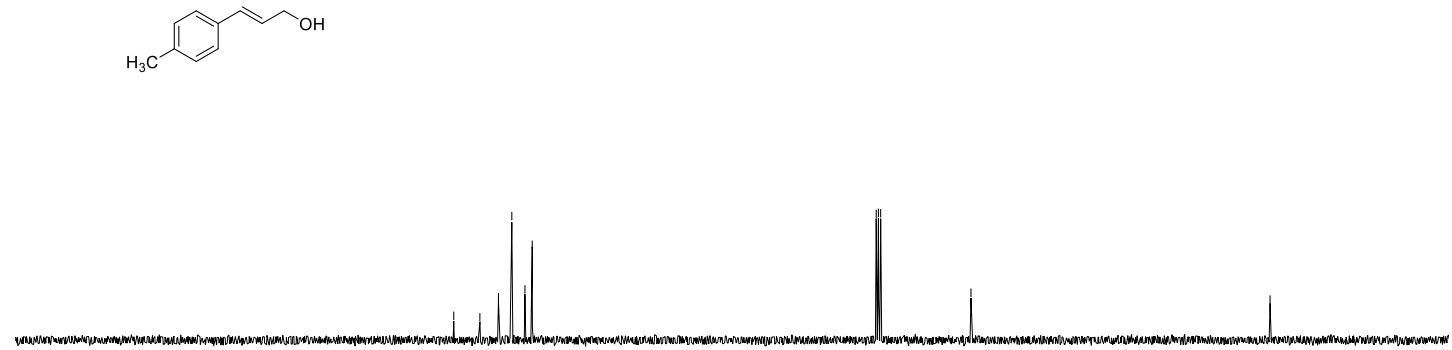

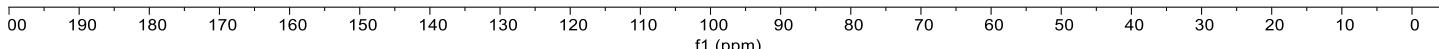

2ac; ${ }^{1} \mathrm{H} \mathrm{NMR} ; \mathrm{CDCl}_{3} ; 400 \mathrm{MHz}$ 


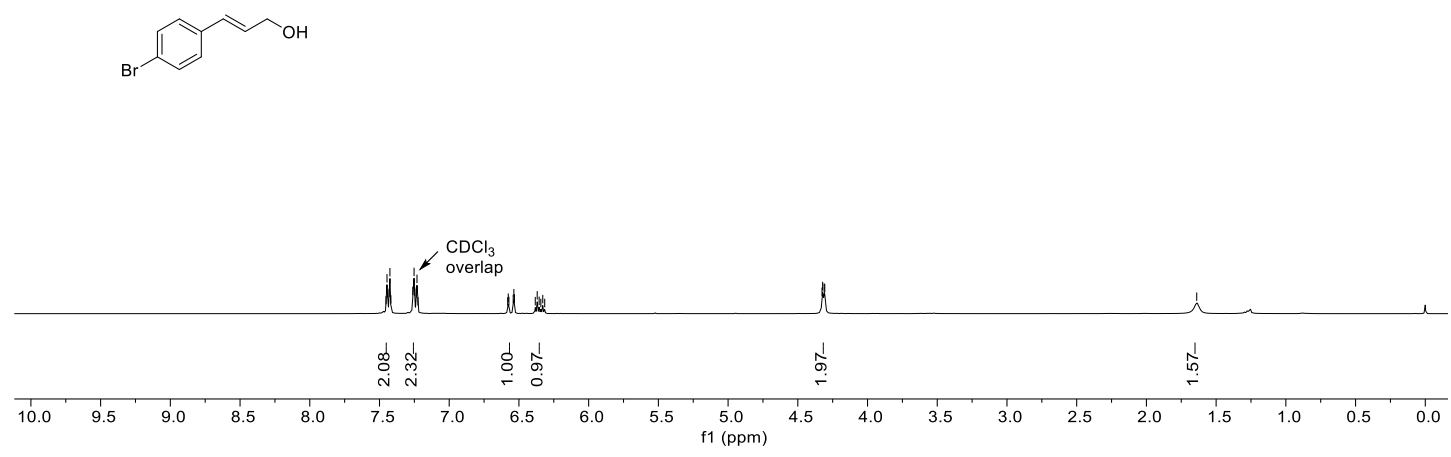

2ac; ${ }^{13} \mathrm{C} \mathrm{NMR} ; \mathrm{CDCl}_{3} ; 101 \mathrm{MHz}$

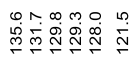

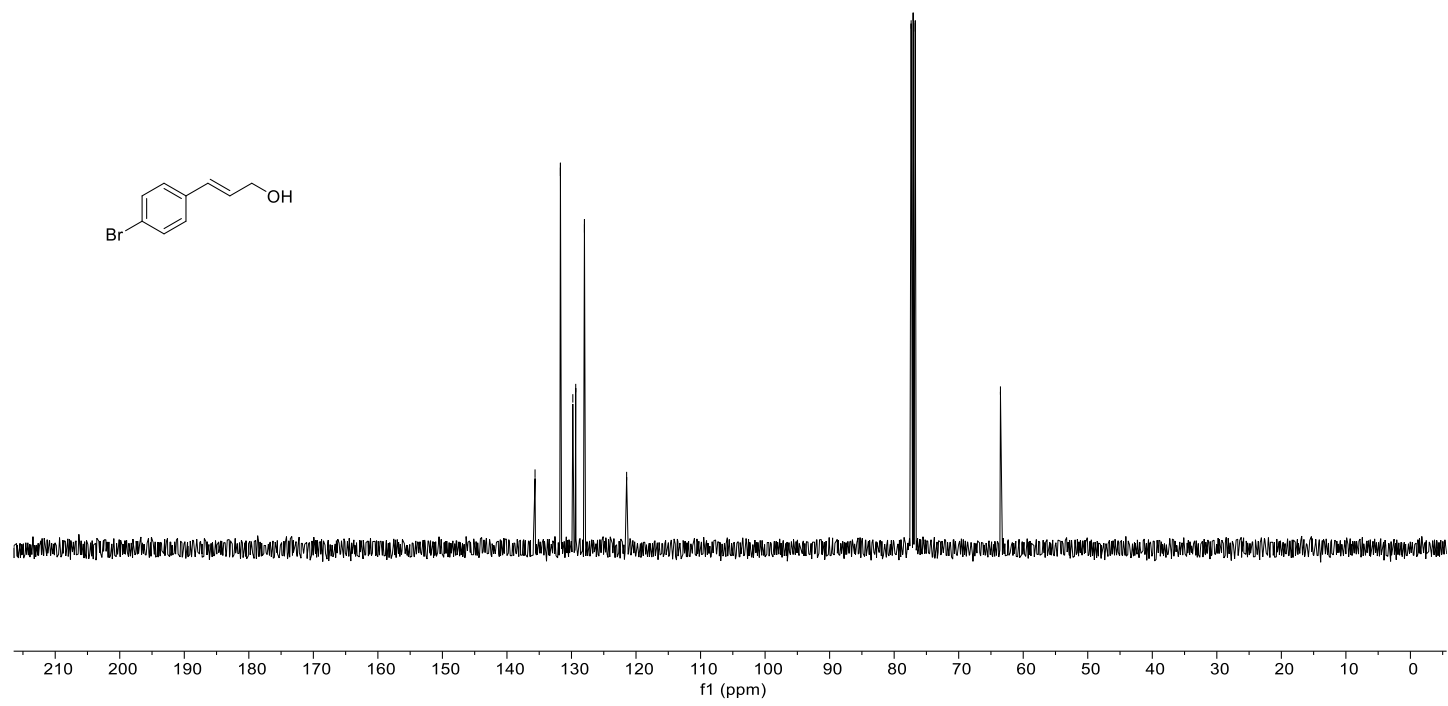

2ad; ${ }^{1} \mathrm{H} \mathrm{NMR} ; \mathrm{CDCl}_{3} ; 400 \mathrm{MHz}$ 
年

$\stackrel{\leftrightarrow}{\stackrel{\leftrightarrow}{~}}$

$\stackrel{i n}{i}$
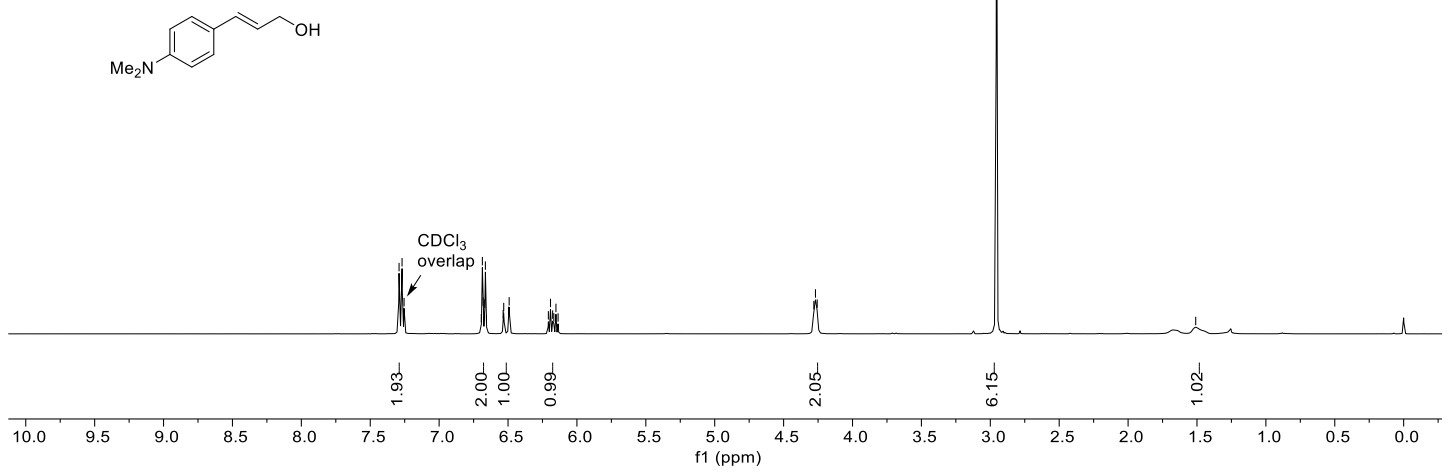

2ad; ${ }^{13} \mathrm{C} \mathrm{NMR} ; \mathrm{CDCl}_{3} ; 101 \mathrm{MHz}$
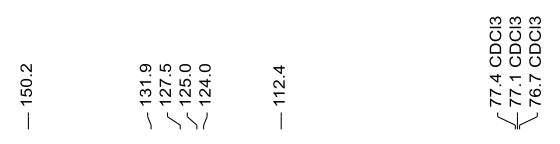

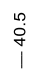
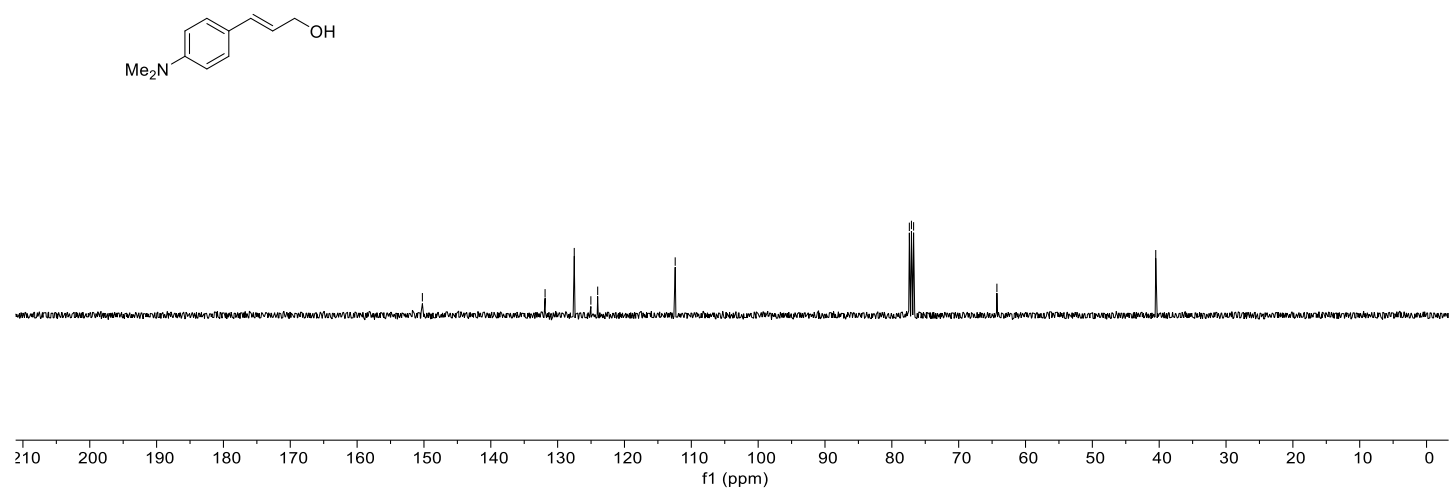

2ae; ${ }^{1} \mathrm{H} \mathrm{NMR} ; \mathrm{CDCl}_{3} ; 400 \mathrm{MHz}$

59 / 150 

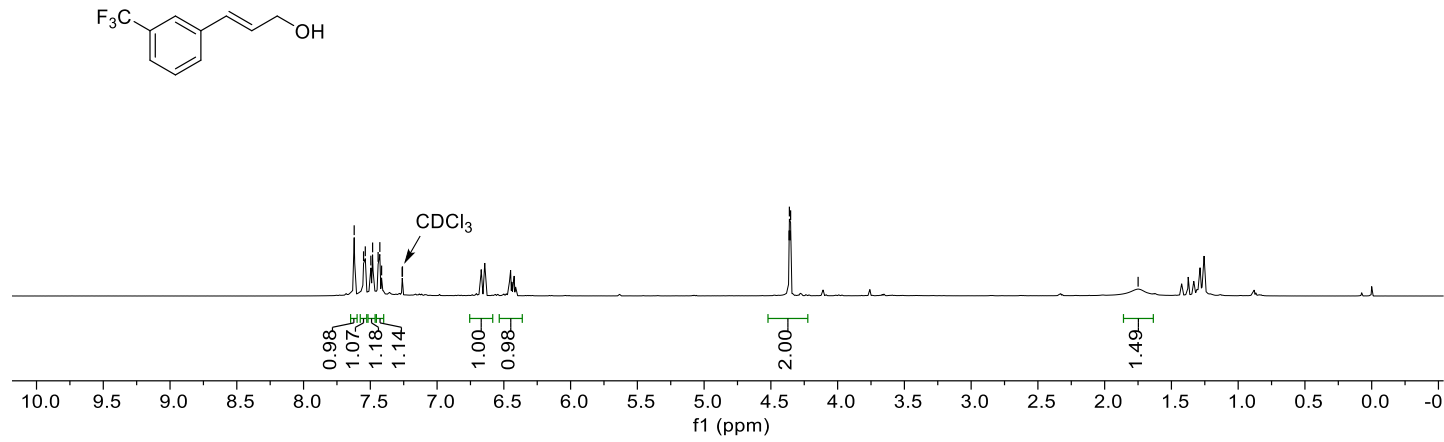

2af; ${ }^{19} \mathrm{~F}$ NMR; $\mathrm{CDCl}_{3} ; 565 \mathrm{MHz}$

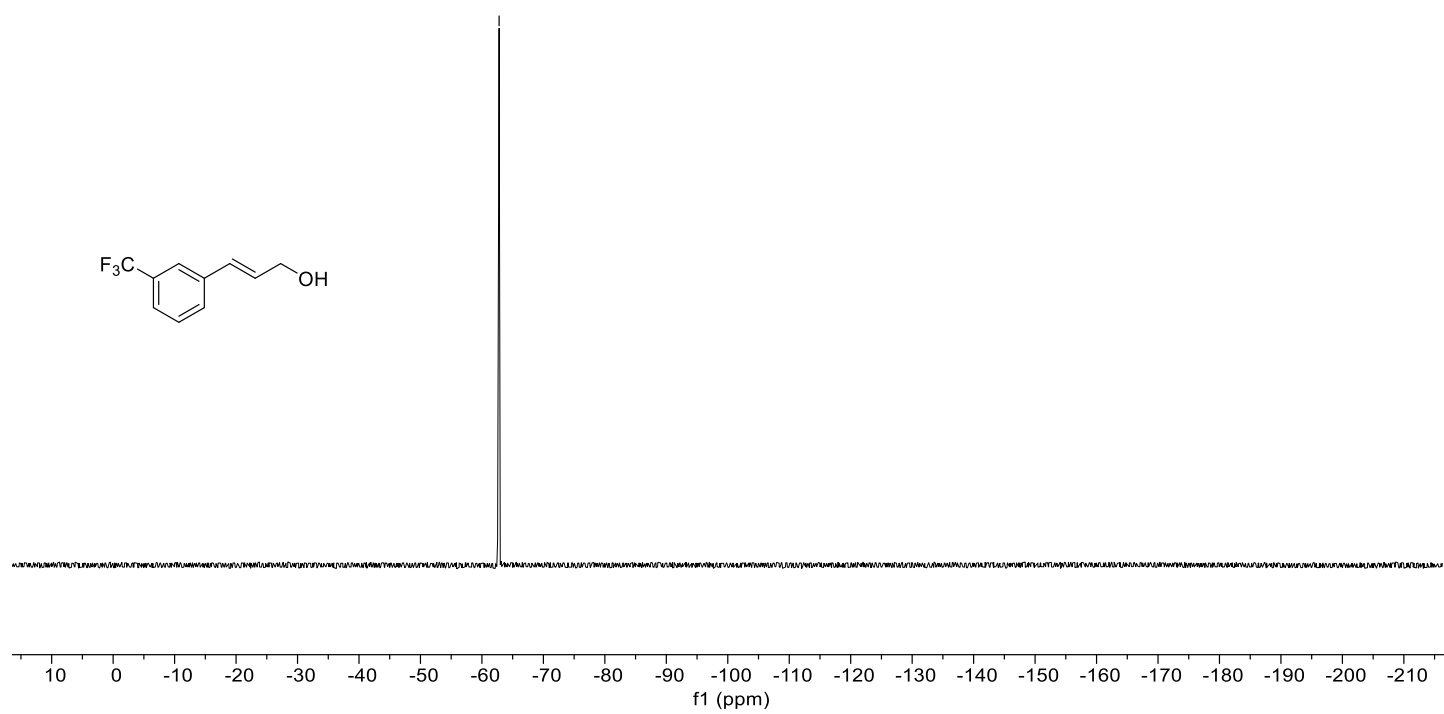

$$
\begin{aligned}
& 0 \\
& 0 \\
& 0 \\
& 0 \\
& 1
\end{aligned}
$$

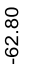

2af; ${ }^{13} \mathrm{C} \mathrm{NMR} ; \mathrm{CDCl}_{3} ; 151 \mathrm{MHz}$ 

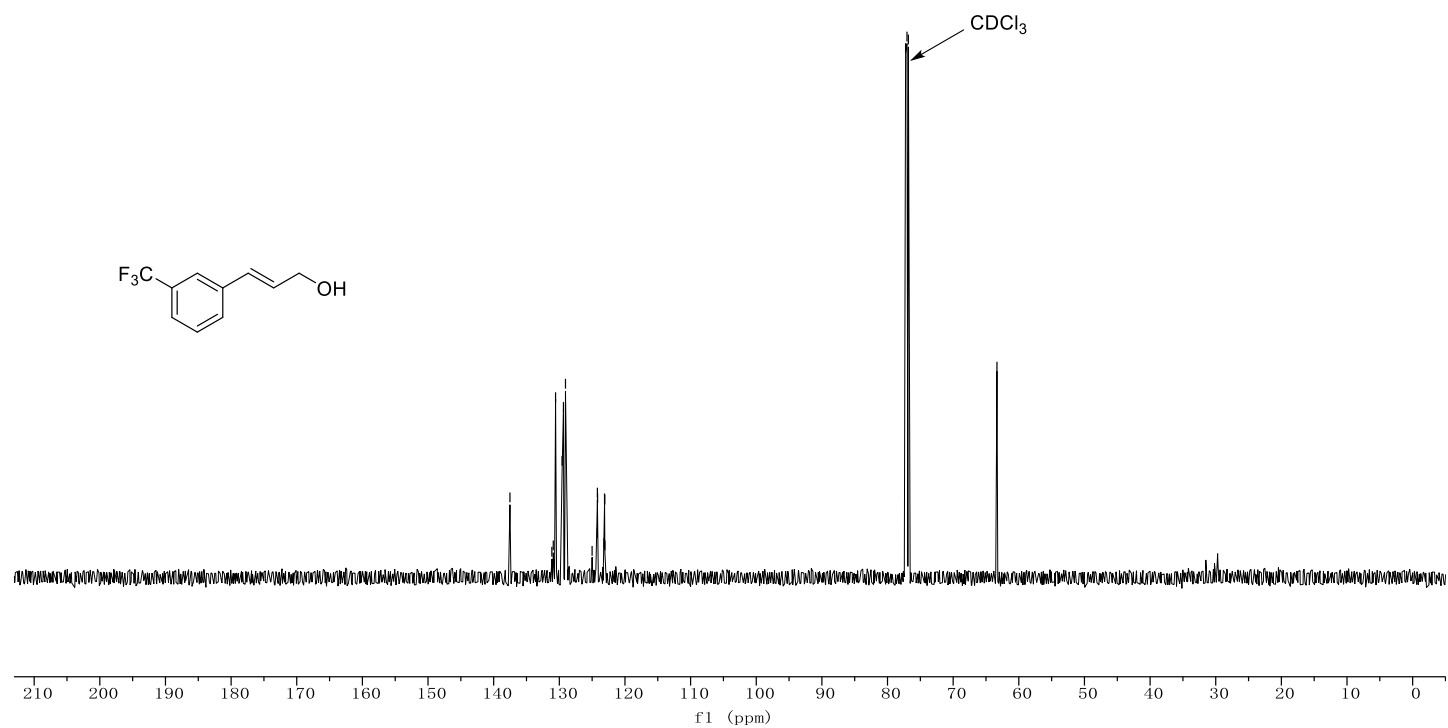

2ag; ${ }^{1} \mathrm{H} \mathrm{NMR} ; \mathrm{CDCl}_{3} ; 400 \mathrm{MHz}$

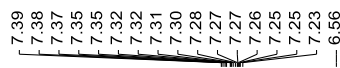

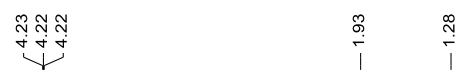

$\curvearrowright \mathrm{CH}_{\mathrm{CH}_{3}}^{\mathrm{OH}}$

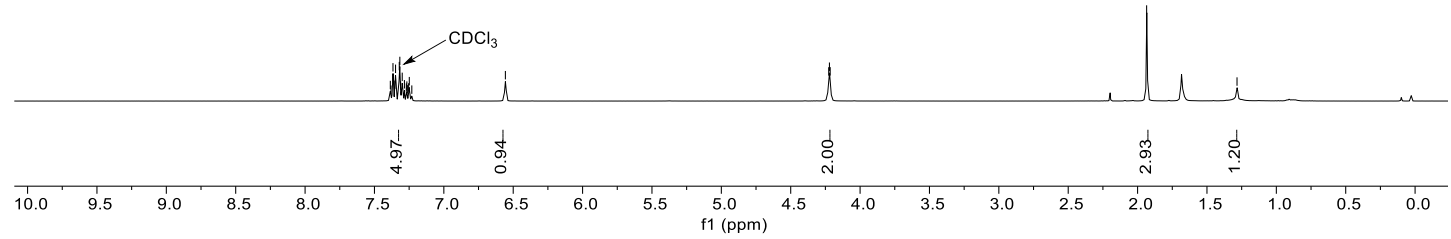

2ag; ${ }^{13} \mathrm{C} \mathrm{NMR} ; \mathrm{CDCl}_{3} ; 101 \mathrm{MHz}$ 
(1) $\mathrm{CH}_{\mathrm{CH}_{3}} \mathrm{OH}$

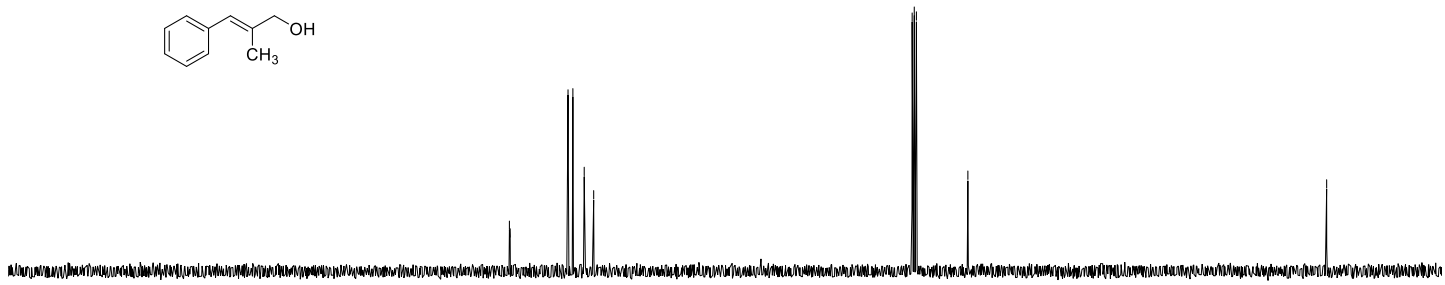

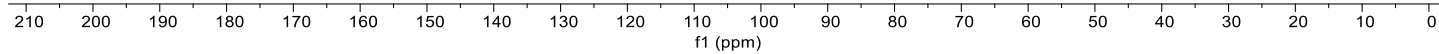

2ah; ${ }^{1} \mathrm{H} \mathrm{NMR} ; \mathrm{CDCl}_{3} ; 400 \mathrm{MHz}$

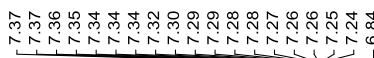

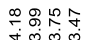

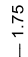
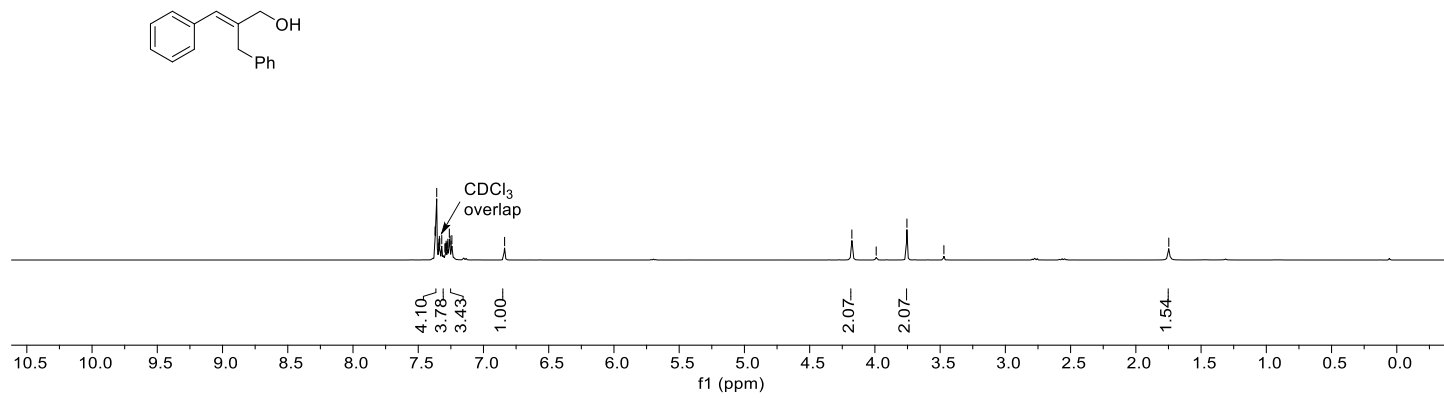

2ah; ${ }^{13} \mathrm{C} \mathrm{NMR} ; \mathrm{CDCl}_{3} ; 101 \mathrm{MHz}$ 

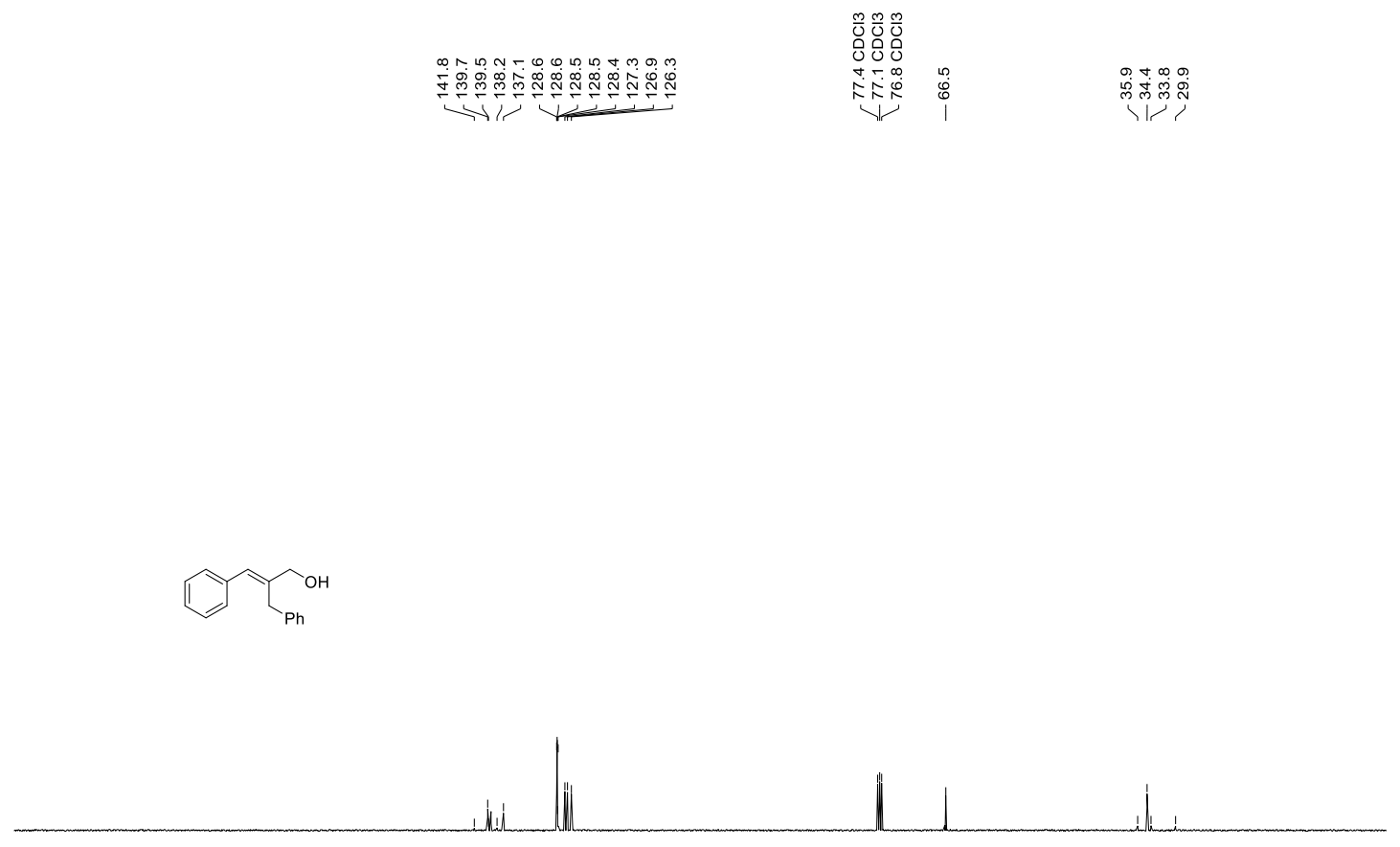

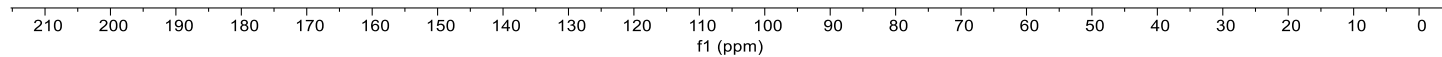

2ai; ${ }^{1} \mathrm{H} \mathrm{NMR}$; $\mathrm{CDCl}_{3}, 400 \mathrm{MHz}$

$\sqrt{\ln }$

$\overbrace{\substack{0 \\ 0}}^{1}$
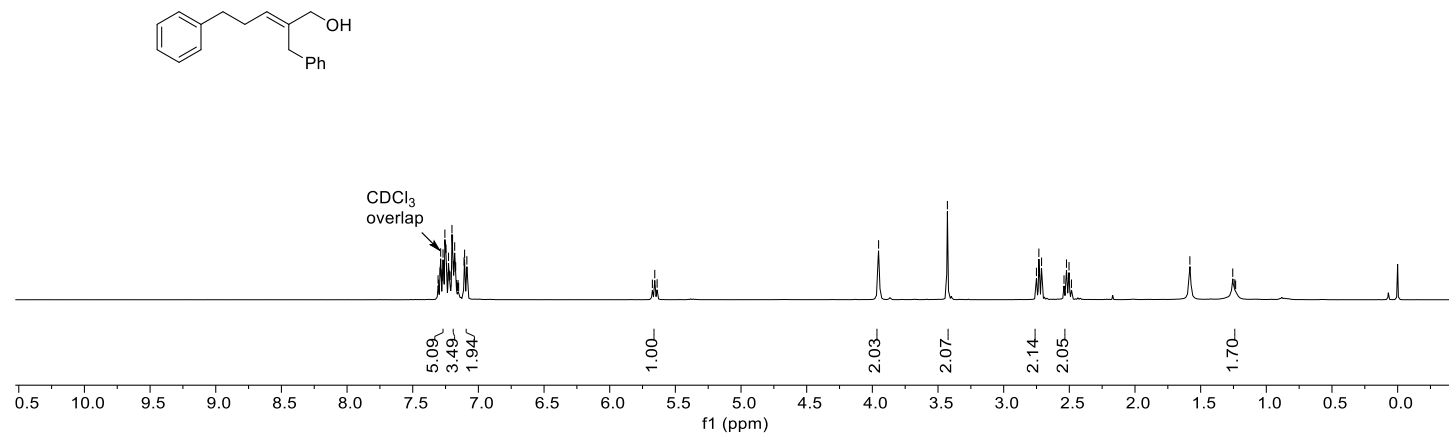

2ai; ${ }^{13} \mathrm{C} \mathrm{NMR} ; \mathrm{CDCl}_{3}, 101 \mathrm{MHz}$ 


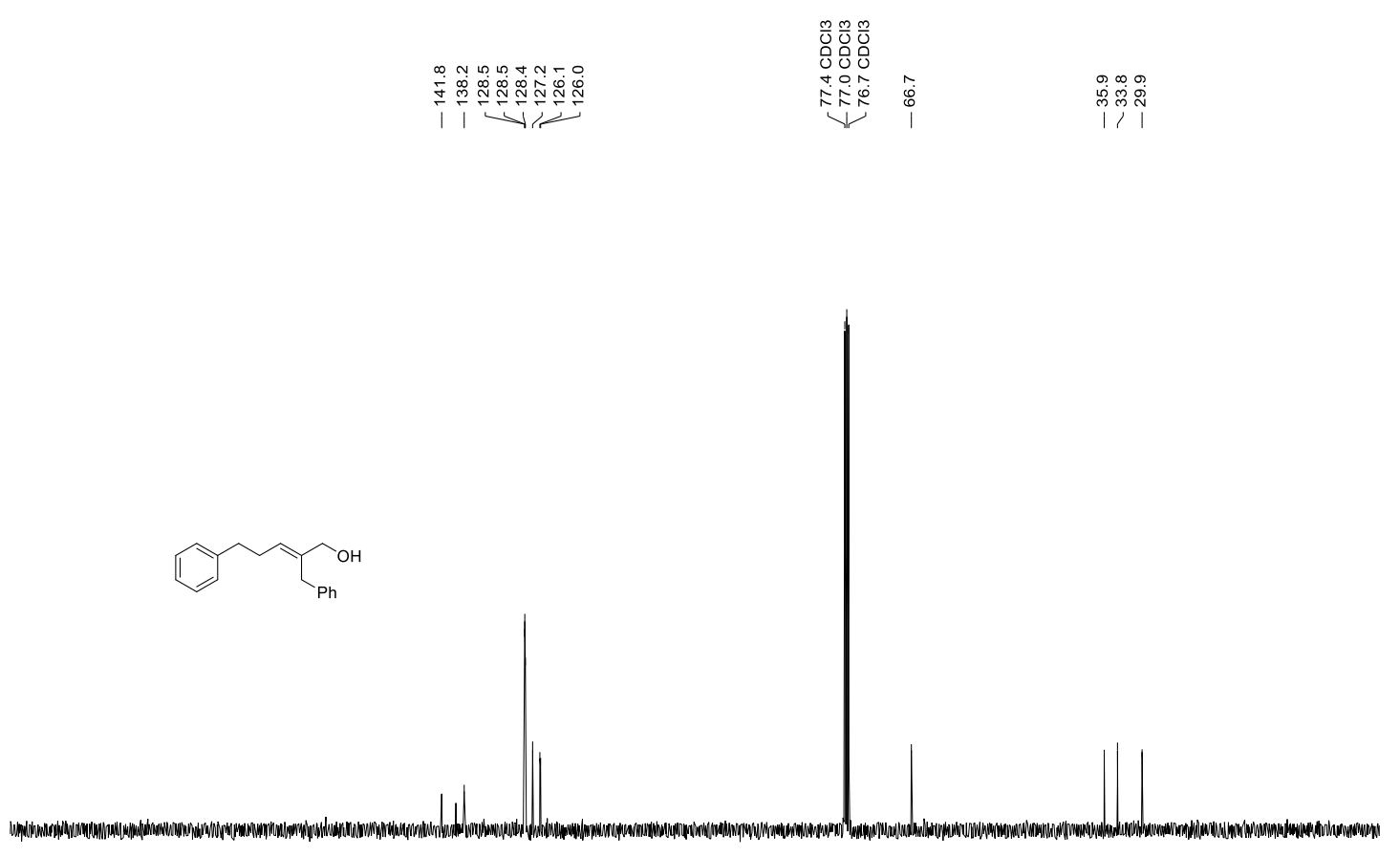

$\begin{array}{lllllllllllllllllllllll}1 & 10 & 200 & 190 & 180 & 170 & 160 & 150 & 140 & 130 & 120 & 110 & 100 & 10 & 80 & 70 & 60 & \frac{1}{1} 10 & 40 & 30 & 20 & 10 & 0\end{array}$

2aj; ${ }^{1} \mathrm{H} \mathrm{NMR}$; $\mathrm{CDCl}_{3} ; 600 \mathrm{MHz}$

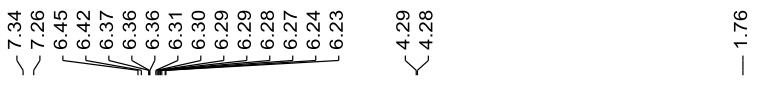
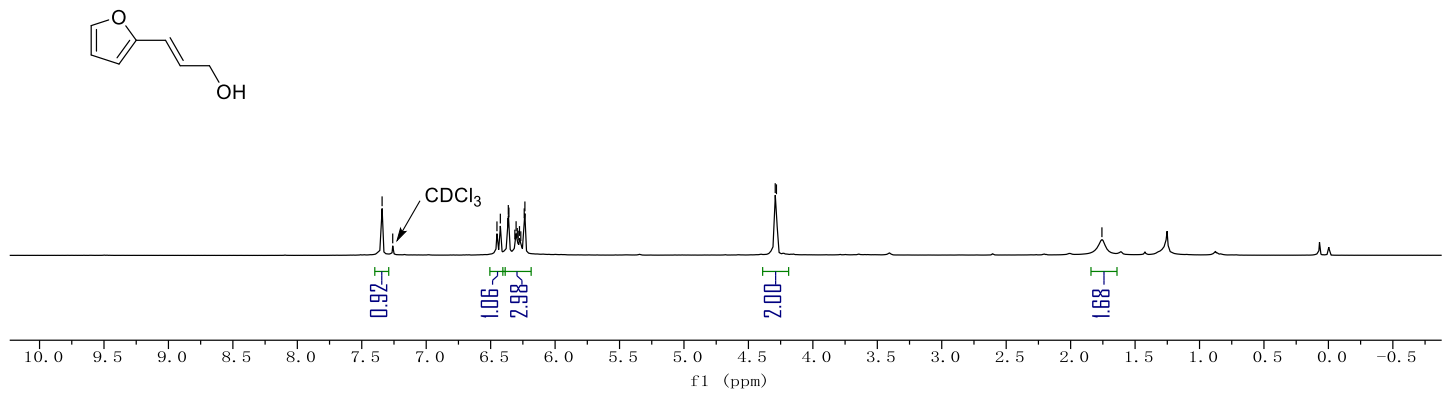

2aj; ${ }^{13} \mathrm{C} \mathrm{NMR} ; \mathrm{CDCl}_{3} ; 151 \mathrm{MHz}$ 


$$
\text { i }
$$

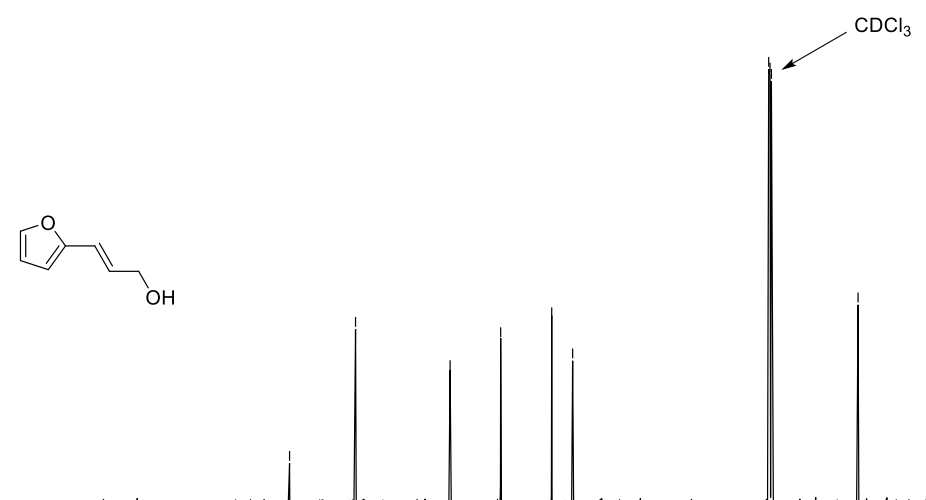

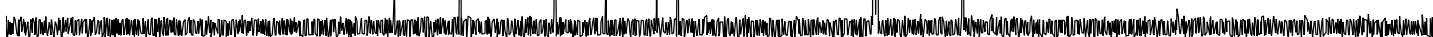

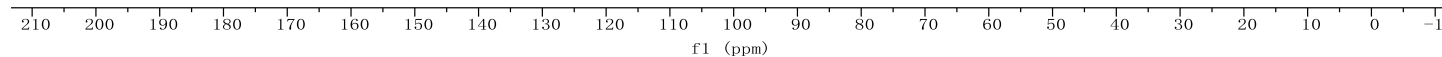

2ak; ${ }^{1} \mathrm{H} \mathrm{NMR} ; \mathrm{CDCl}_{3} ; 400 \mathrm{MHz}$

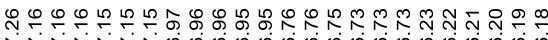

r r r
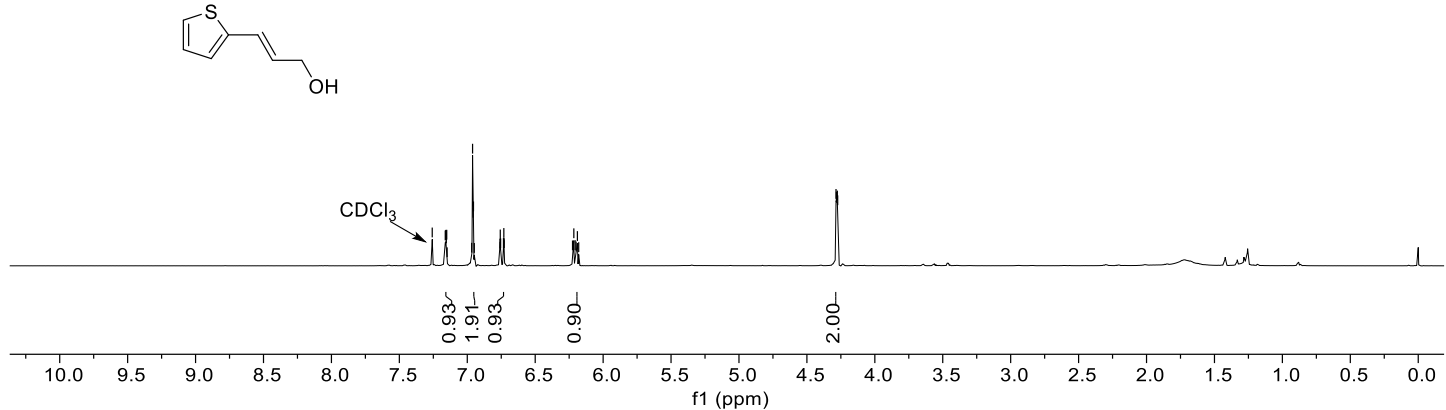

2ak; ${ }^{13} \mathrm{C} \mathrm{NMR} ; \mathrm{CDCl}_{3} ; 101 \mathrm{MHz}$ 


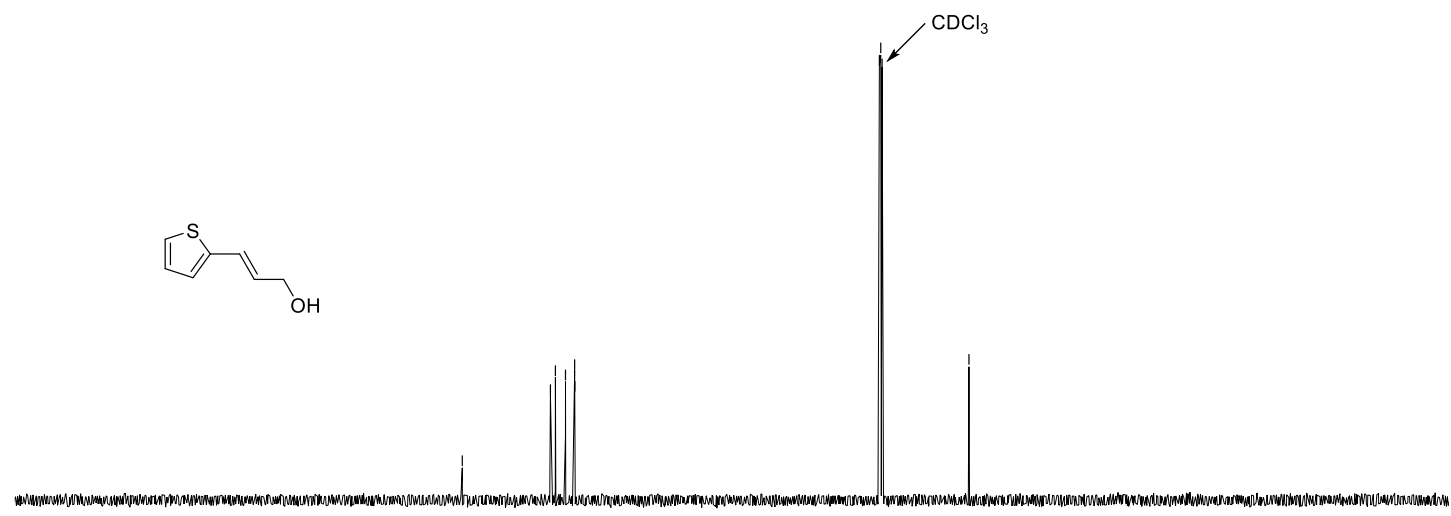

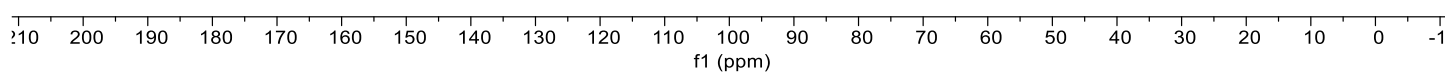

2al; ${ }^{1} \mathrm{H} \mathrm{NMR} ; \mathrm{CDCl}_{3} ; 600 \mathrm{MHz}$

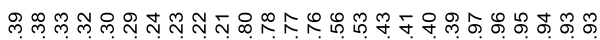

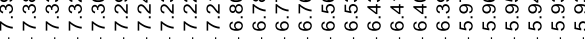

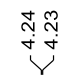

$\stackrel{\infty}{i}$
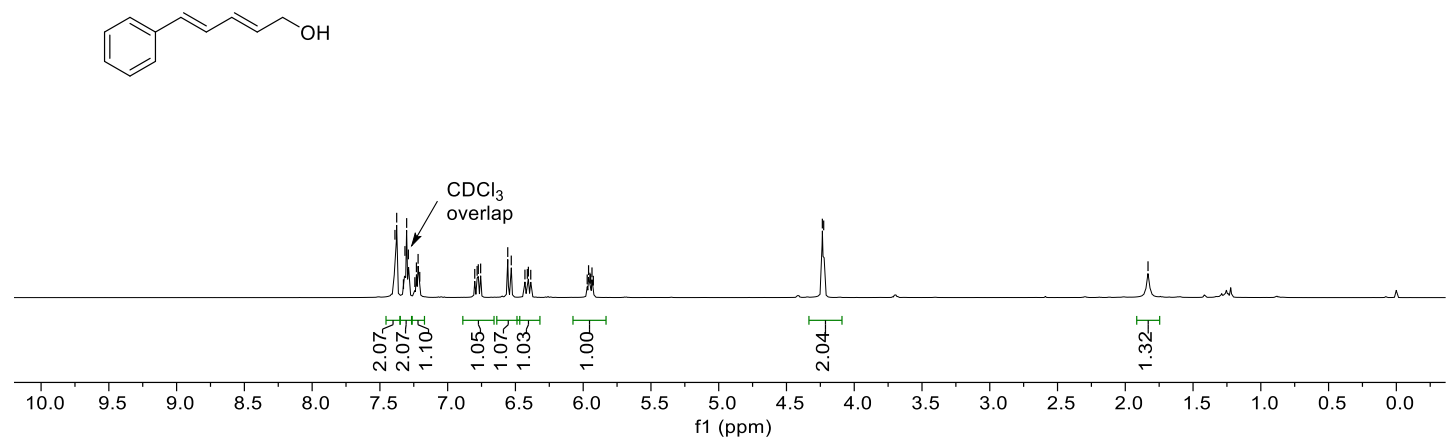

2al; ${ }^{13} \mathrm{C} \mathrm{NMR} ; \mathrm{CDCl}_{3} ; 151 \mathrm{MHz}$ 

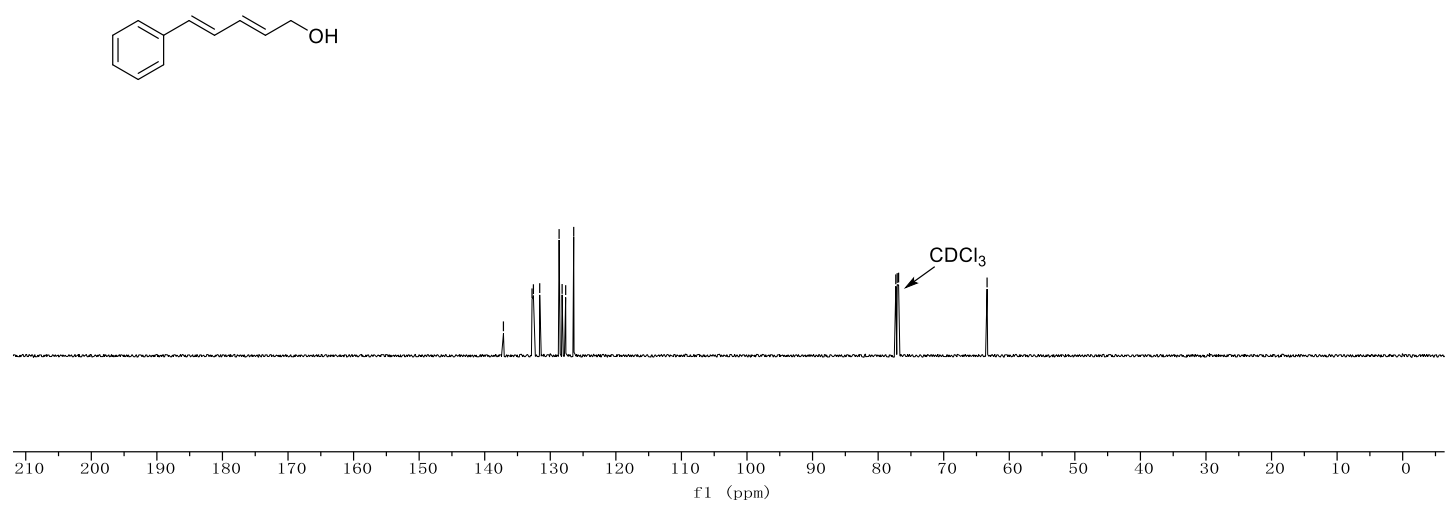

2am; ${ }^{1} \mathrm{H} \mathrm{NMR} ; \mathrm{CDCl}_{3} ; 400 \mathrm{MHz}$

象

$\mathrm{H}_{3} \mathrm{C} \overbrace{\mathrm{CH}}^{\mathrm{CH}_{3} \mathrm{H}_{3}}$

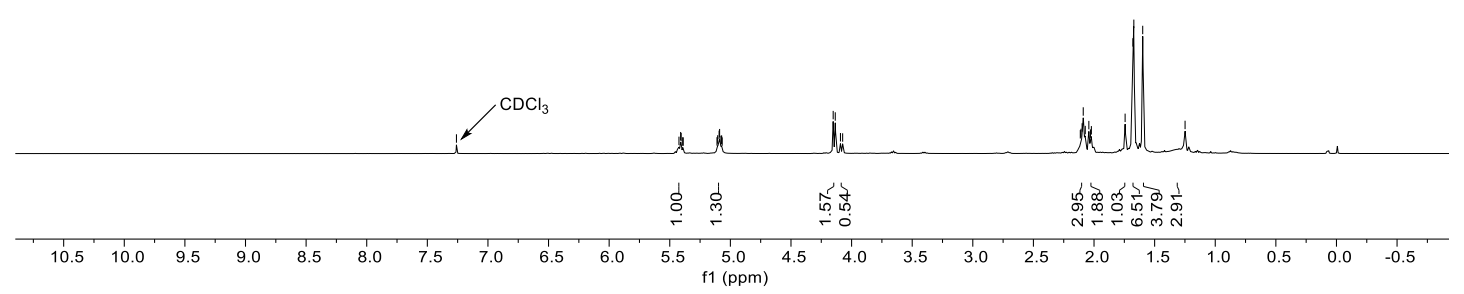

2am; ${ }^{13} \mathrm{C} \mathrm{NMR} ; \mathrm{CDCl}_{3} ; 101 \mathrm{MHz}$ 


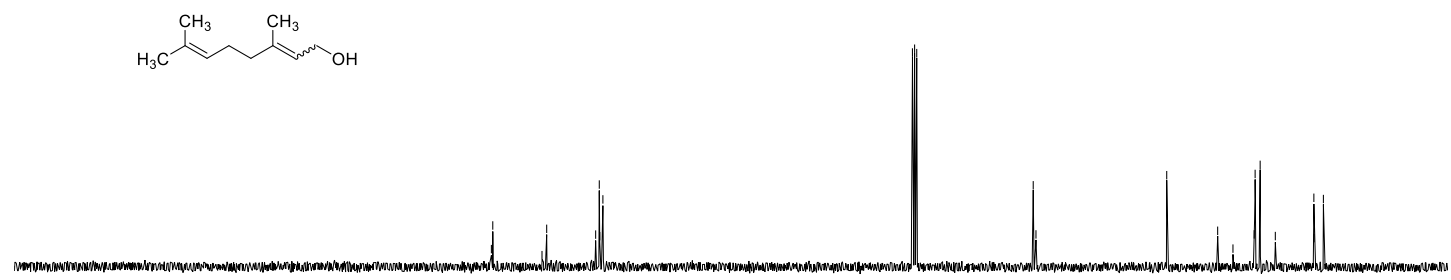

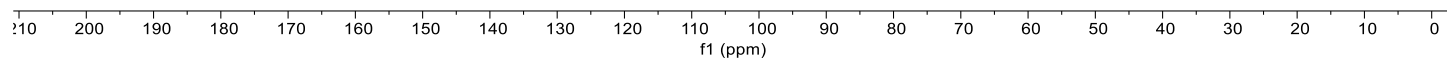

2an; ${ }^{1} \mathrm{H}$ NMR; $\mathrm{CDCl}_{3} ; 400 \mathrm{MHz}$

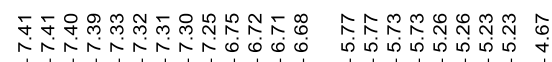
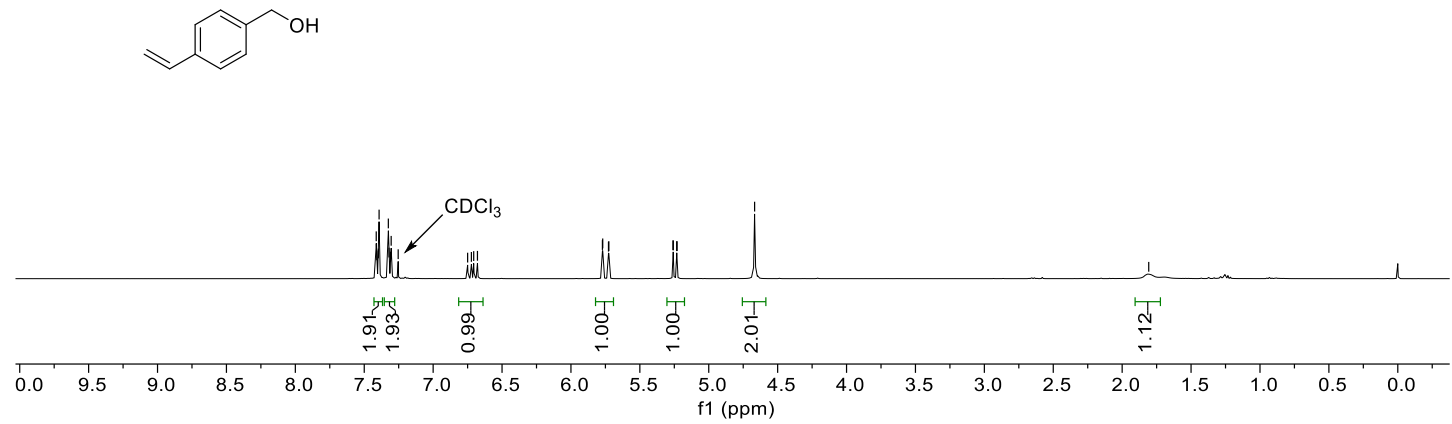

2an; ${ }^{13} \mathrm{C} \mathrm{NMR} ; \mathrm{CDCl}_{3} ; 101 \mathrm{MHz}$ 


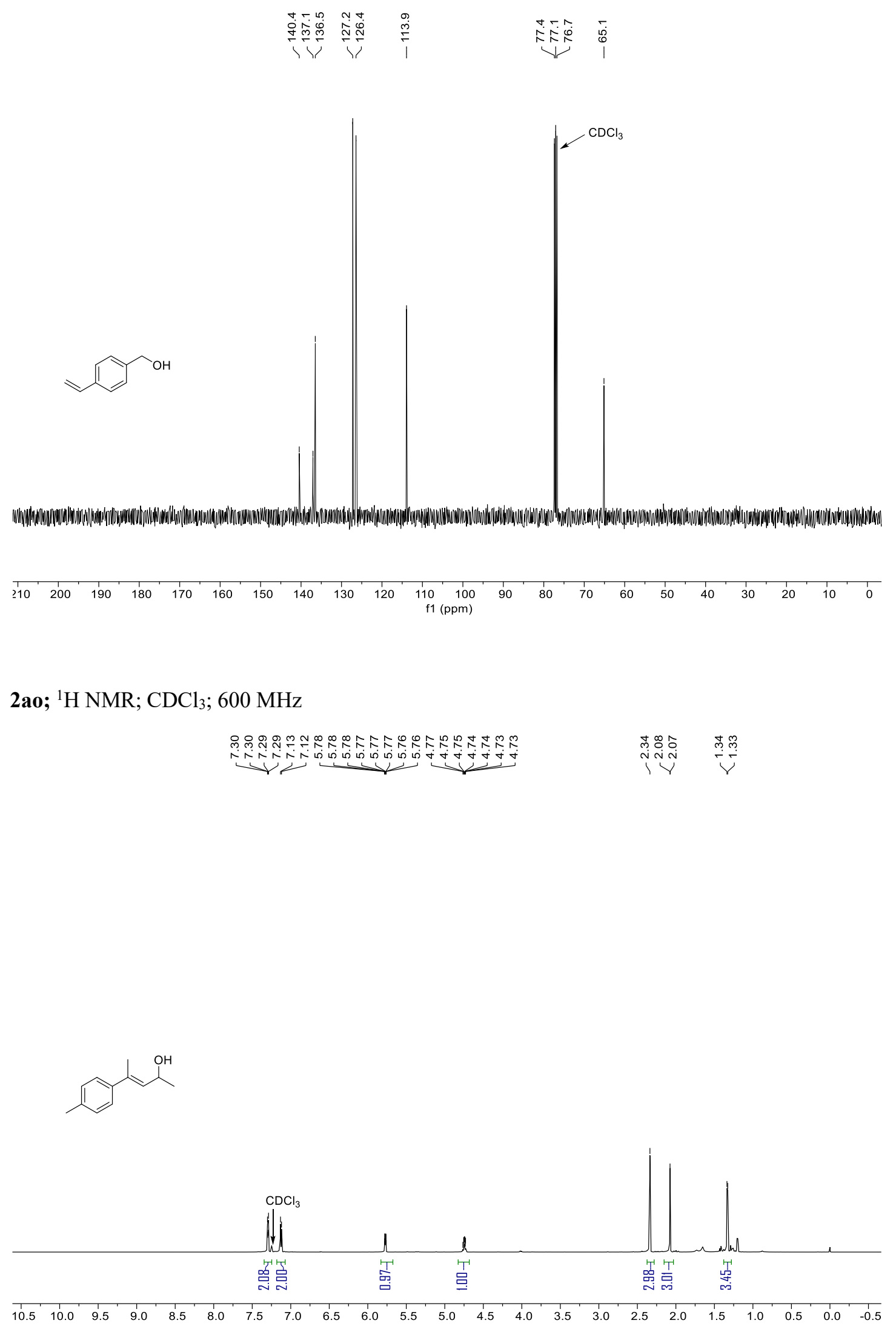

2ao; ${ }^{13} \mathrm{C} \mathrm{NMR} ; \mathrm{CDCl}_{3} ; 151 \mathrm{MHz}$ 


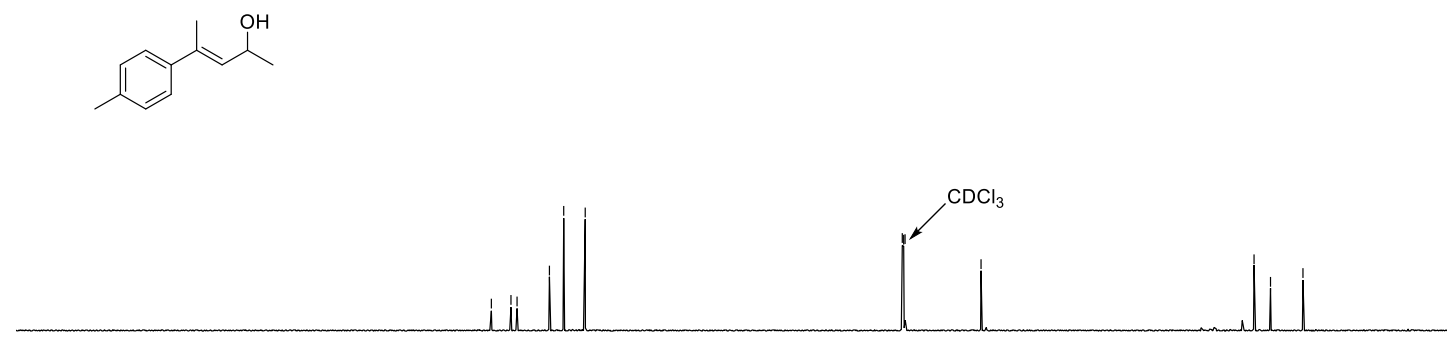

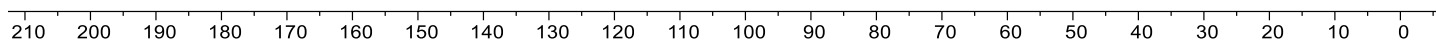

2ap; ${ }^{1} \mathrm{H} \mathrm{NMR} ; \mathrm{CDCl}_{3} ; 400 \mathrm{MHz}$

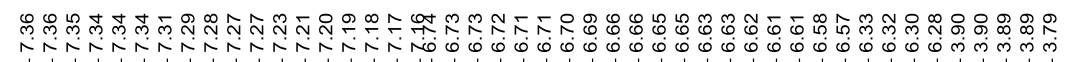
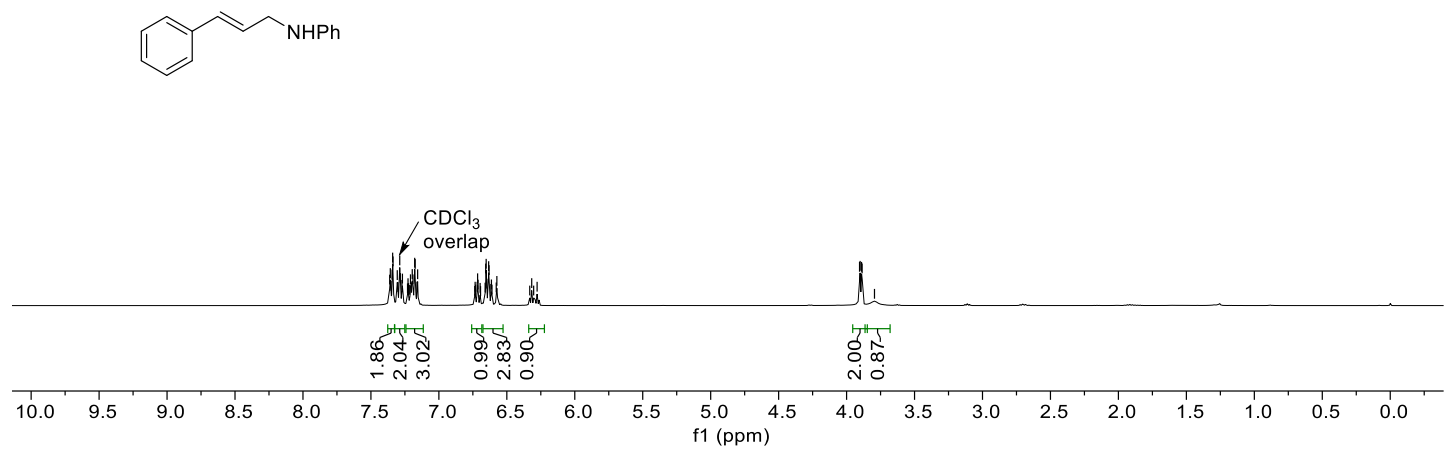

2ap; ${ }^{13} \mathrm{C} \mathrm{NMR} ; \mathrm{CDCl}_{3} ; 101 \mathrm{MHz}$ 


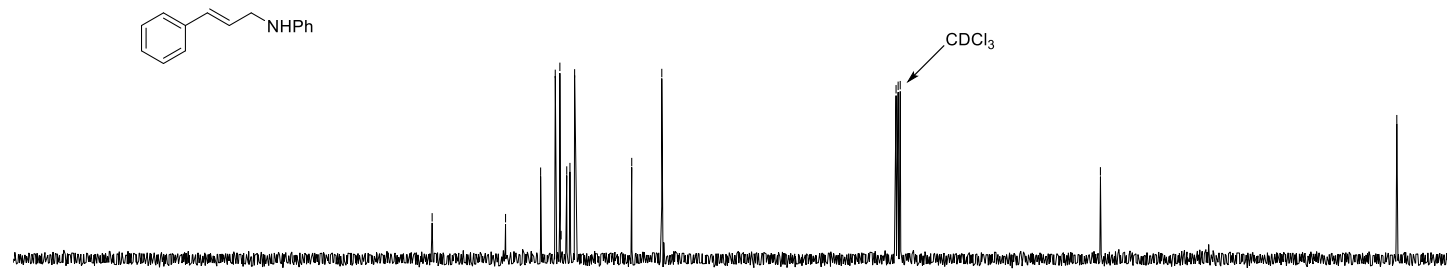

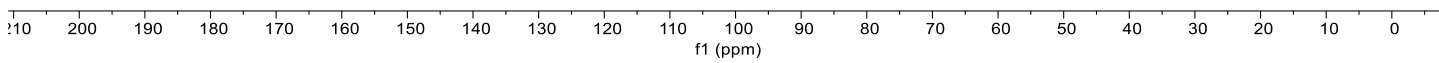

2aq; ${ }^{1} \mathrm{H} \mathrm{NMR} ; \mathrm{CDCl}_{3} ; 400 \mathrm{MHz}$

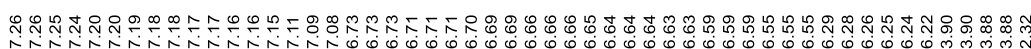

NNNNMNNKNNMNN

$\mathrm{H}_{3} \mathrm{C}$

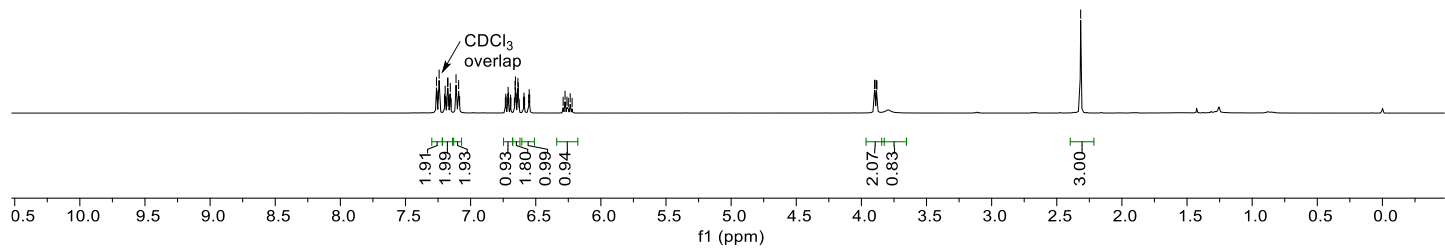

2aq; ${ }^{13} \mathrm{C} \mathrm{NMR} ; \mathrm{CDCl}_{3} ; 101 \mathrm{MHz}$ 


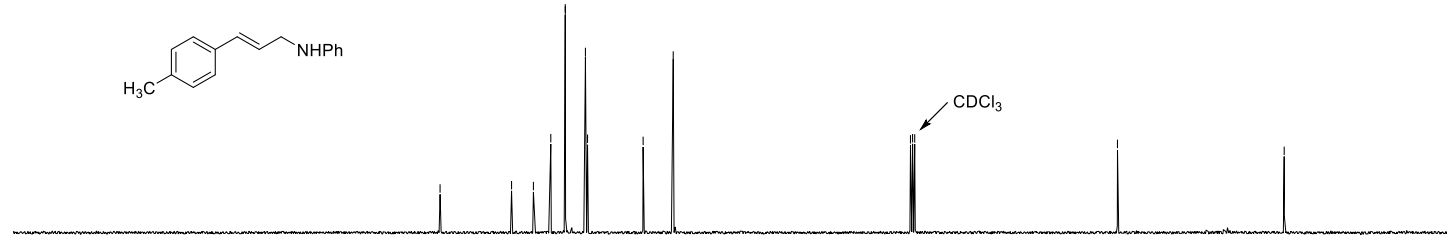

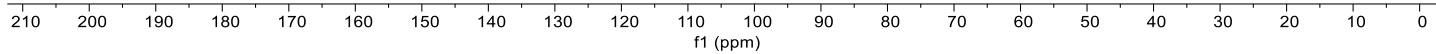

2ar; ${ }^{1} \mathrm{H}$ NMR; $\mathrm{CDCl}_{3} ; 400 \mathrm{MHz}$

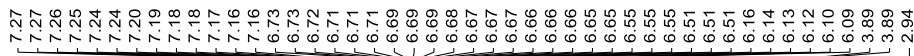

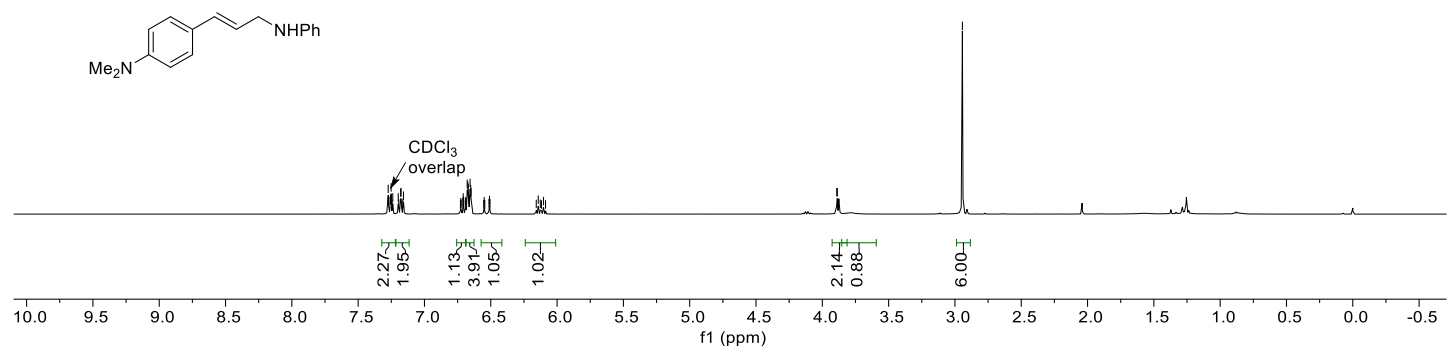

2ar; ${ }^{13} \mathrm{C} \mathrm{NMR} ; \mathrm{CDCl}_{3} ; 101 \mathrm{MHz}$ 

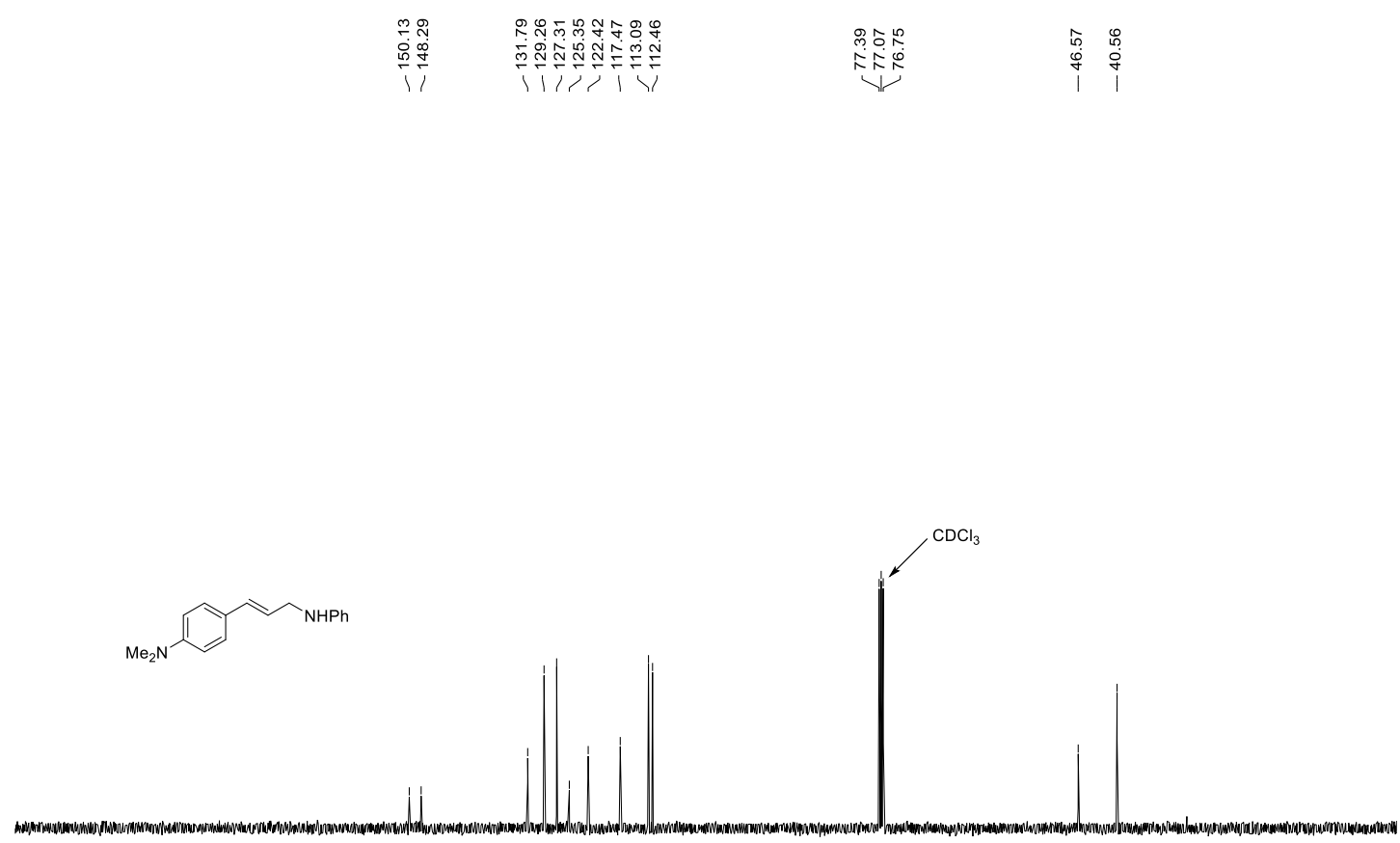

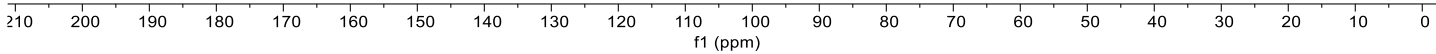

2as; ${ }^{1} \mathrm{H} \mathrm{NMR} ; \mathrm{CDCl}_{3} ; 600 \mathrm{MHz}$

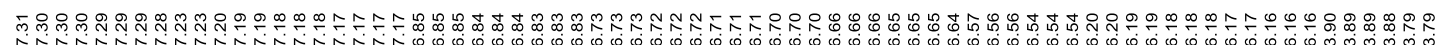

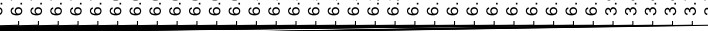

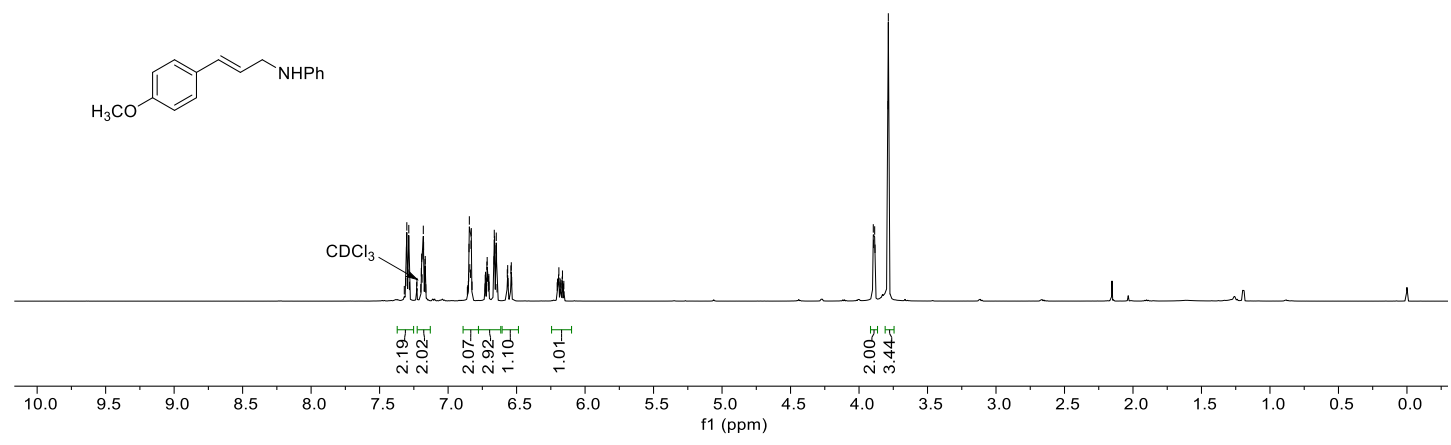

2as; ${ }^{13} \mathrm{C} \mathrm{NMR} ; \mathrm{CDCl}_{3} ; 151 \mathrm{MHz}$ 


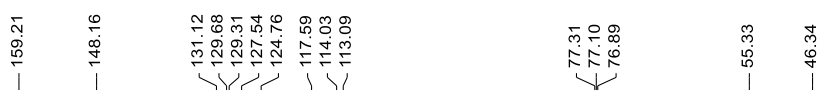

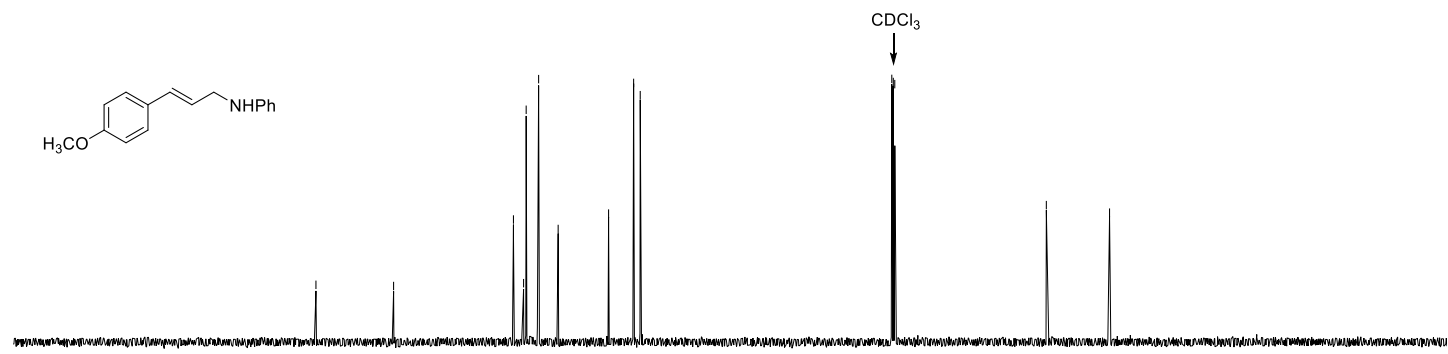

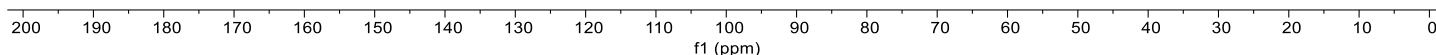

2at; ${ }^{1} \mathrm{H} \mathrm{NMR} ; \mathrm{CDCl}_{3} ; 400 \mathrm{MHz}$

$\underbrace{\underbrace{2}}$
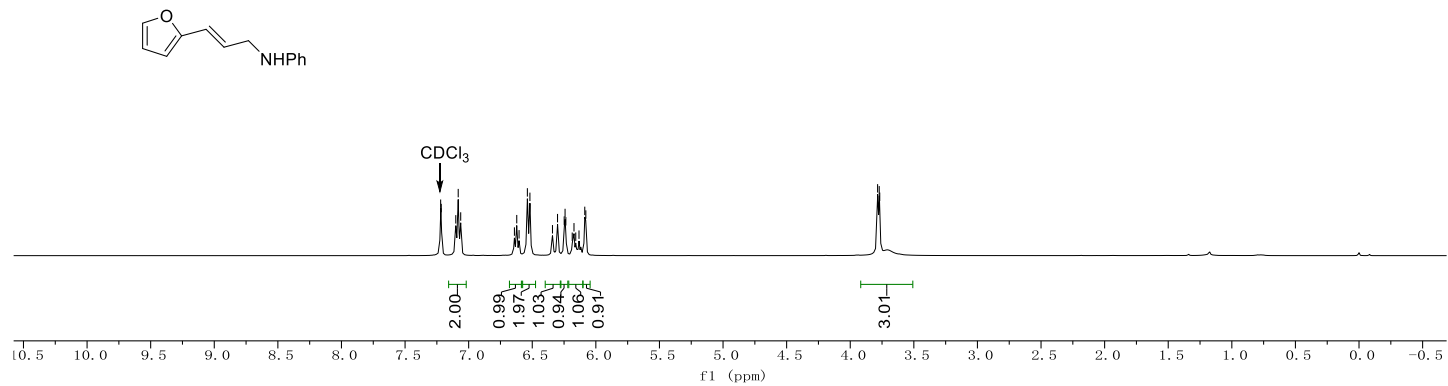

2at; ${ }^{13} \mathrm{C} \mathrm{NMR} ; \mathrm{CDCl}_{3} ; 101 \mathrm{MHz}$ 


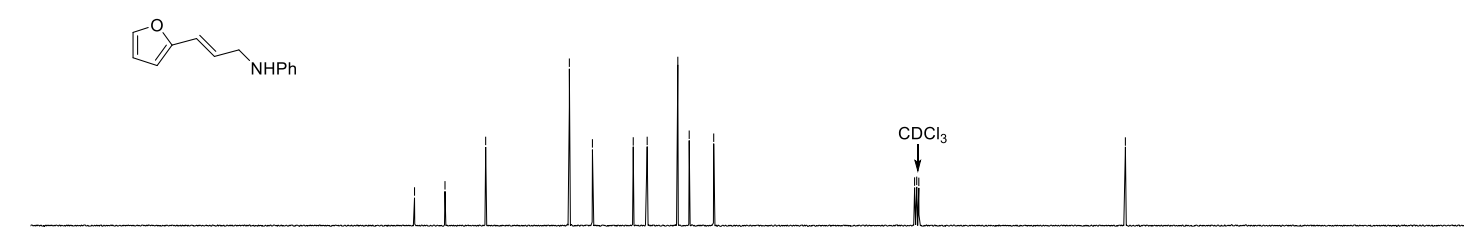

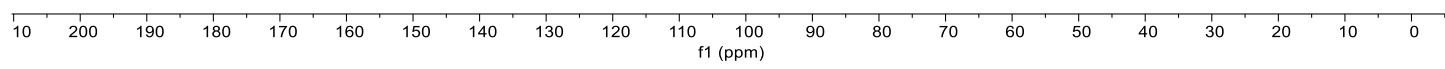

2au; ${ }^{1} \mathrm{H} \mathrm{NMR}$; $\mathrm{CDCl}_{3} ; 600 \mathrm{MHz}$

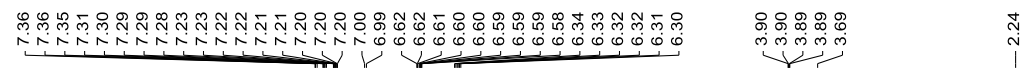

$\overbrace{\mathrm{NHTOI}}$

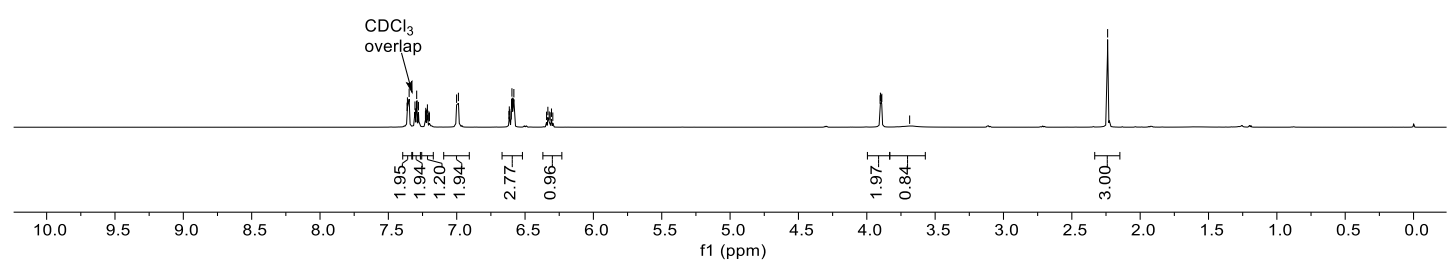

2au; ${ }^{13} \mathrm{C} \mathrm{NMR} ; \mathrm{CDCl}_{3} ; 151 \mathrm{MHz}$ 


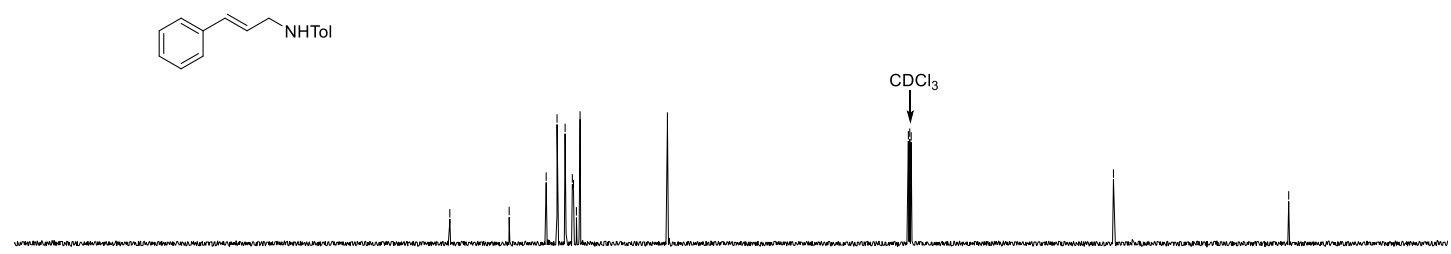

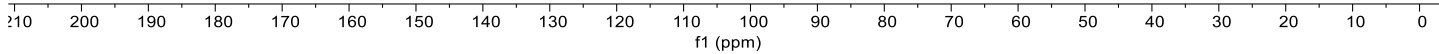

2av; ${ }^{1} \mathrm{H} \mathrm{NMR} ; \mathrm{CDCl}_{3} ; 600 \mathrm{MHz}$

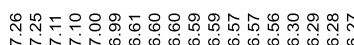

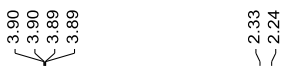

${ }_{\mathrm{H}_{3} \mathrm{C}}^{\mathrm{C}} \mathrm{NHTO}^{\mathrm{N}}$

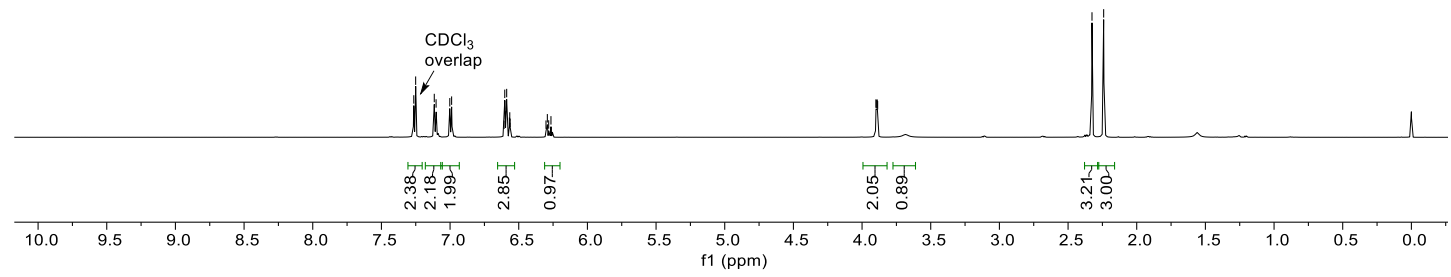

2av; ${ }^{13} \mathrm{C} \mathrm{NMR} ; \mathrm{CDCl}_{3} ; 151 \mathrm{MHz}$ 


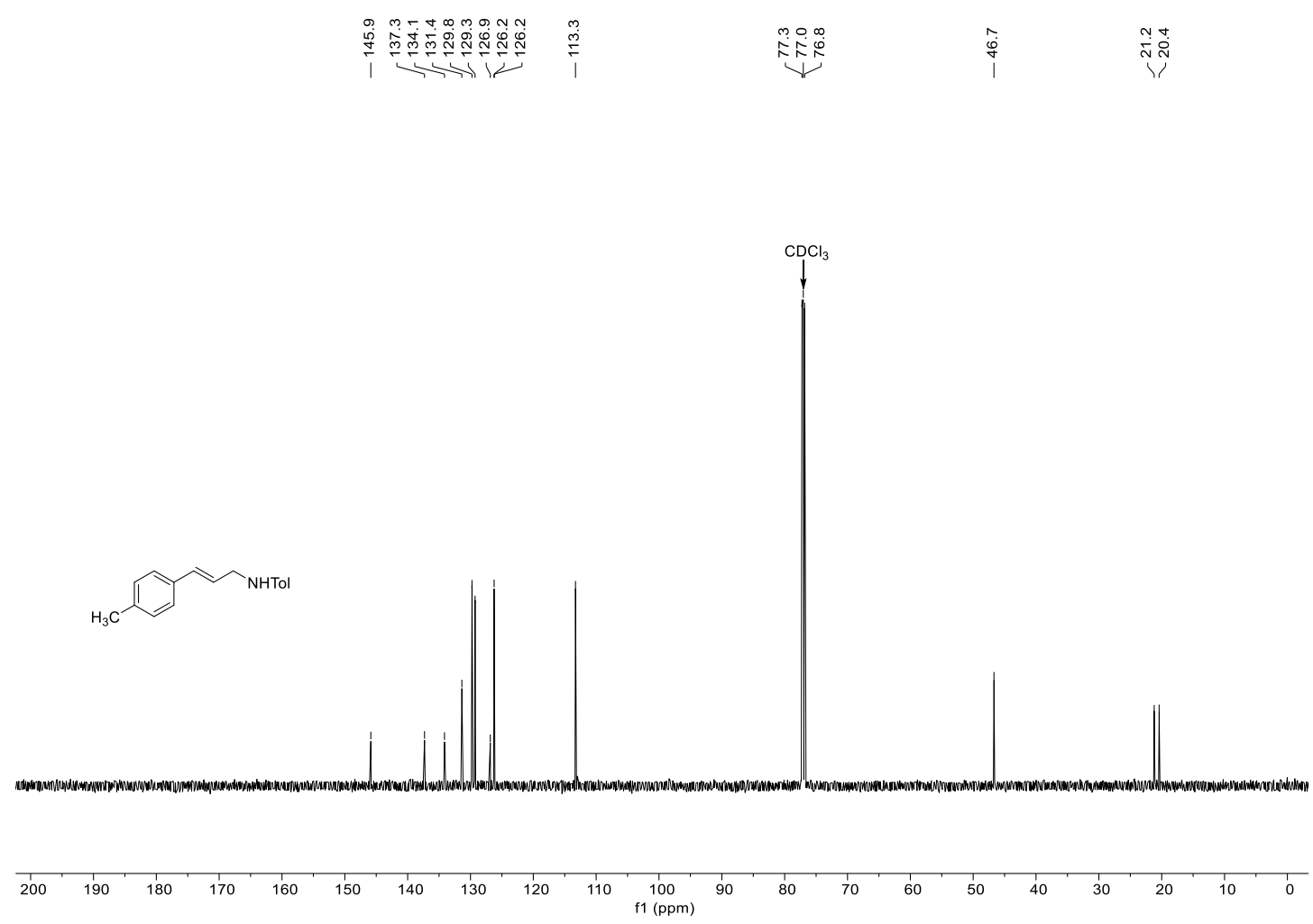

2aw; ${ }^{1} \mathrm{H} \mathrm{NMR} ; \mathrm{CDCl}_{3} ; 400 \mathrm{MHz}$

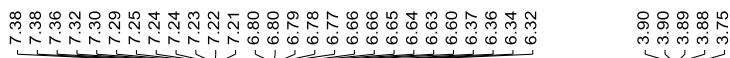

$\overbrace{H}^{O M e}$

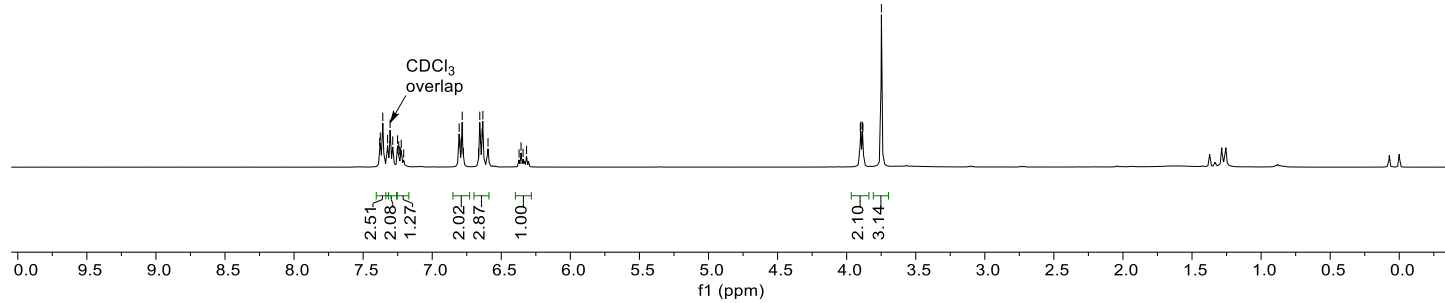

2aw; ${ }^{13} \mathrm{C} \mathrm{NMR} ; \mathrm{CDCl}_{3} ; 101 \mathrm{MHz}$ 


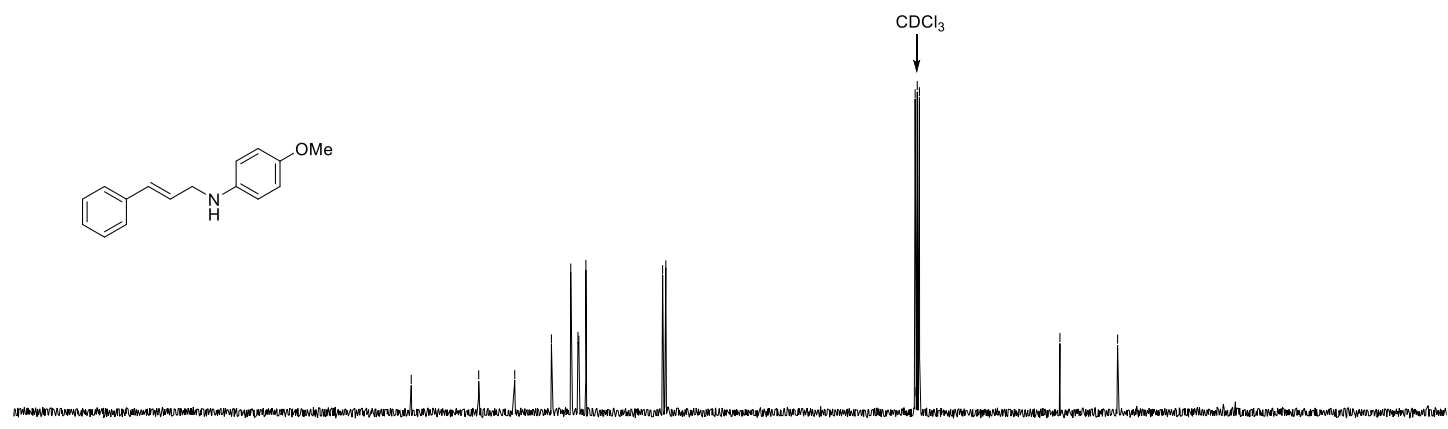

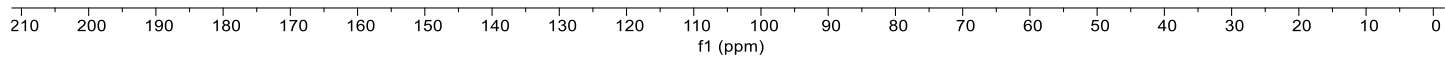

2ba; ${ }^{1} \mathrm{H} \mathrm{NMR} ; \mathrm{CDCl}_{3} ; 400 \mathrm{MHz}$

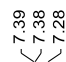

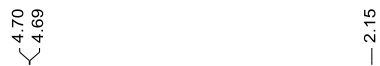

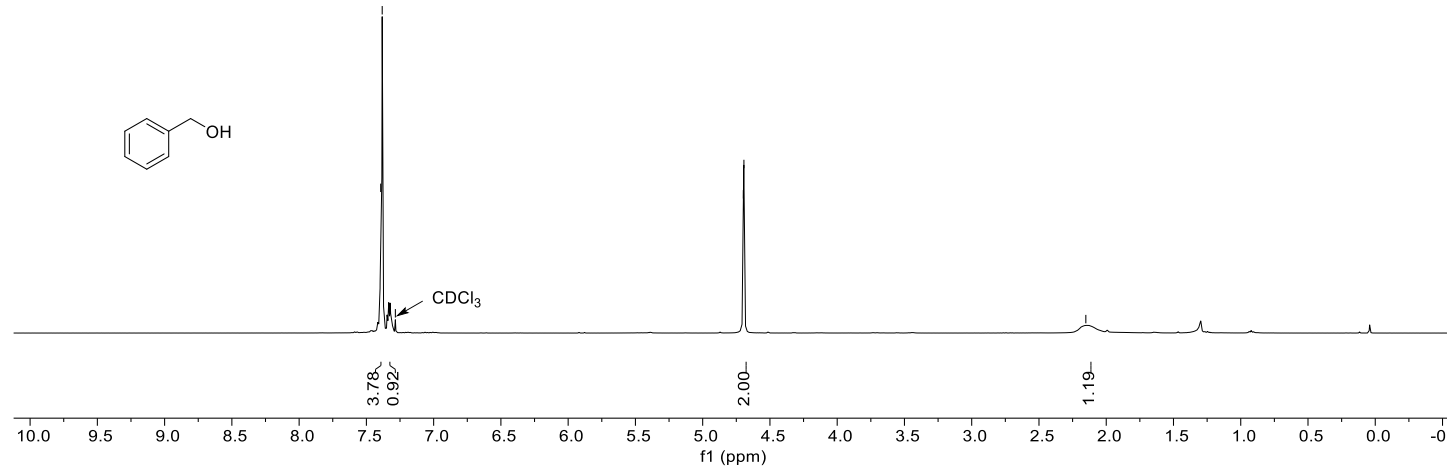

2ba; ${ }^{13} \mathrm{C} \mathrm{NMR} ; \mathrm{CDCl}_{3} ; 101 \mathrm{MHz}$ 


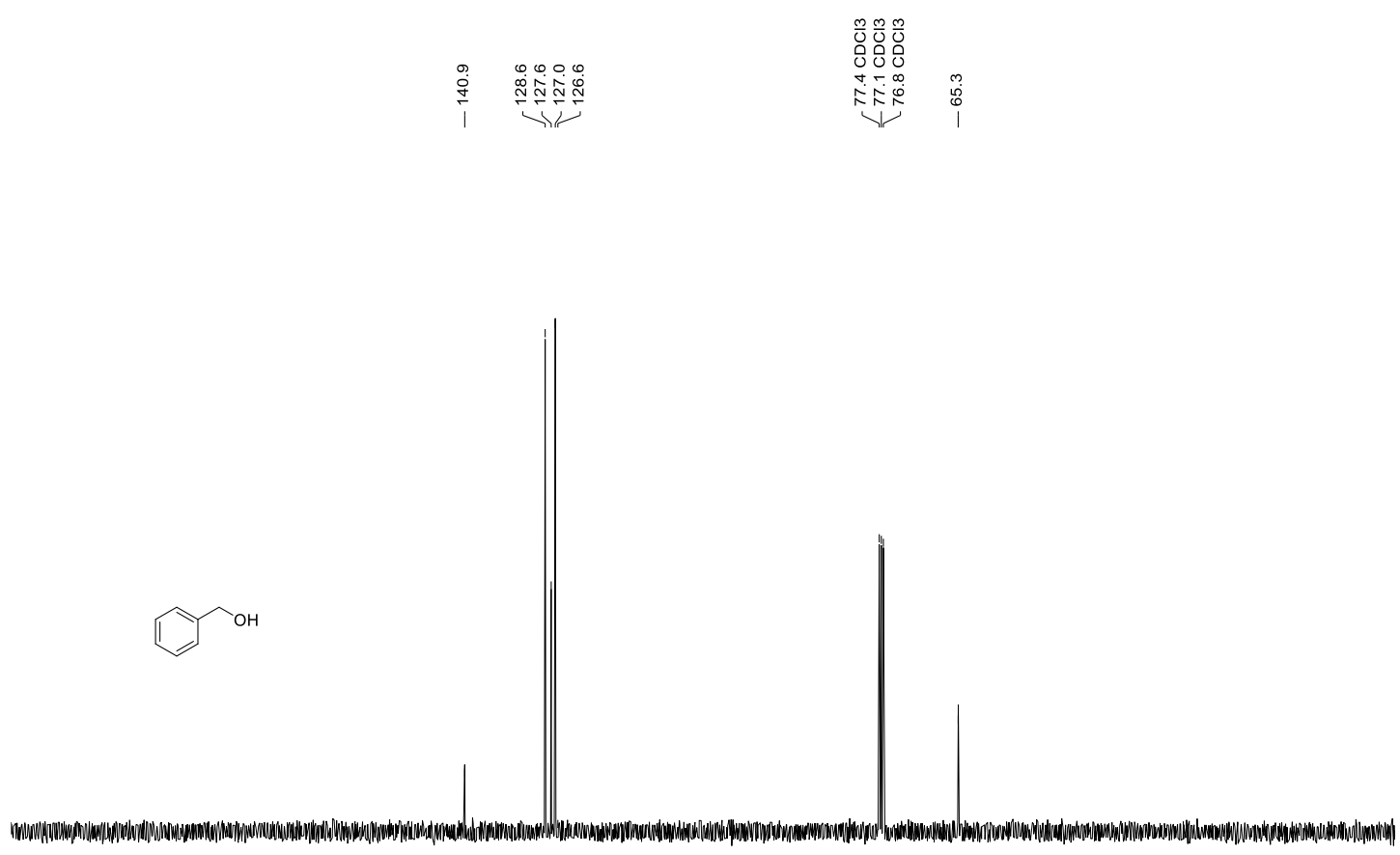

\begin{tabular}{lllllllllllllllllllll}
\hline 10 & 200 & 190 & 180 & 170 & 160 & 150 & 140 & 130 & 120 & $\begin{array}{c}110 \\
\mathrm{f} 1(\mathrm{ppm})\end{array}$ & 90 & 80 & 70 & 60 & 50 & 40 & 30 & 20 & 10 & 0
\end{tabular}

2bb; ${ }^{1} \mathrm{H} \mathrm{NMR} ; \mathrm{CDCl}_{3}, 400 \mathrm{MHz}$

$\underbrace{\underbrace{20}}$

$\overbrace{\mathrm{OH}}$

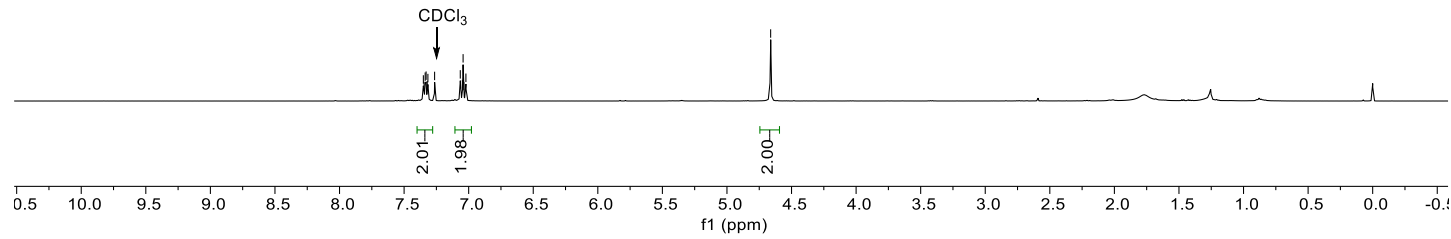

2bb; ${ }^{19} \mathrm{~F} \mathrm{NMR} ; \mathrm{CDCl}_{3} ; 376 \mathrm{MHz}$ 

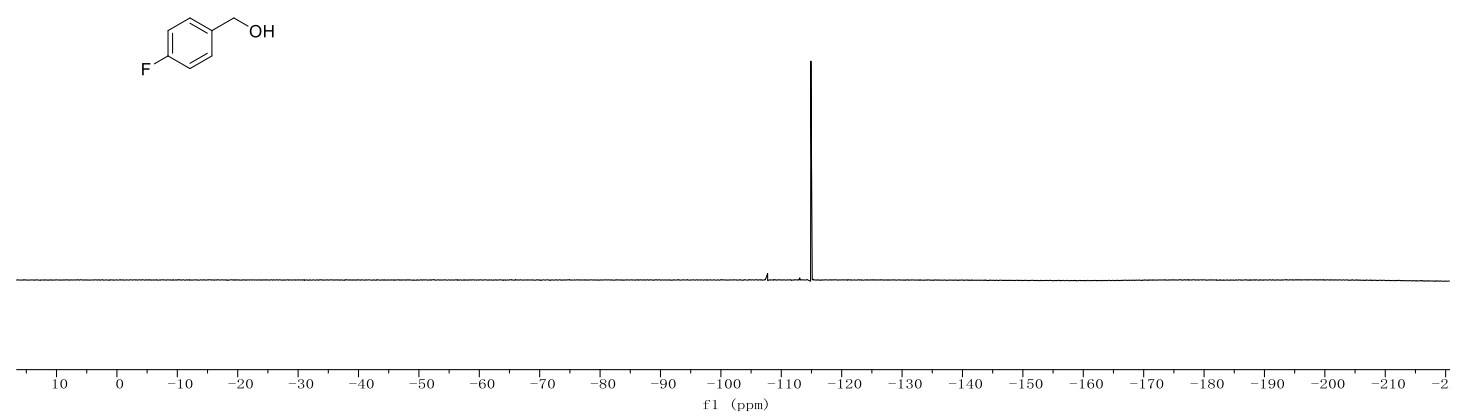

2bb; ${ }^{13} \mathrm{C} \mathrm{NMR} ; \mathrm{CDCl}_{3} ; 101 \mathrm{MHz}$
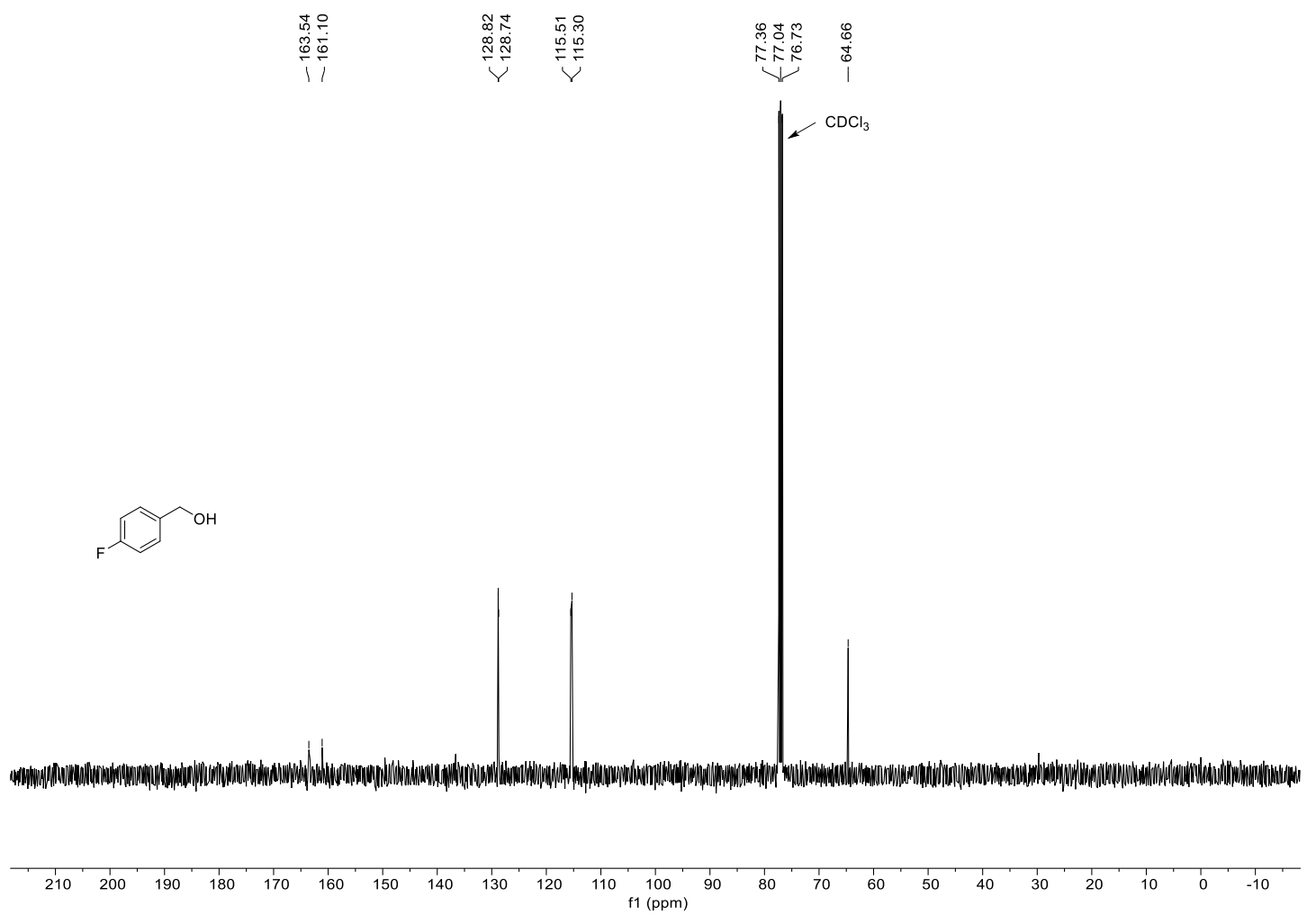

2bc; ${ }^{1} \mathrm{H}$ NMR, $\mathrm{CDCl}_{3} ; 600 \mathrm{MHz}$ 

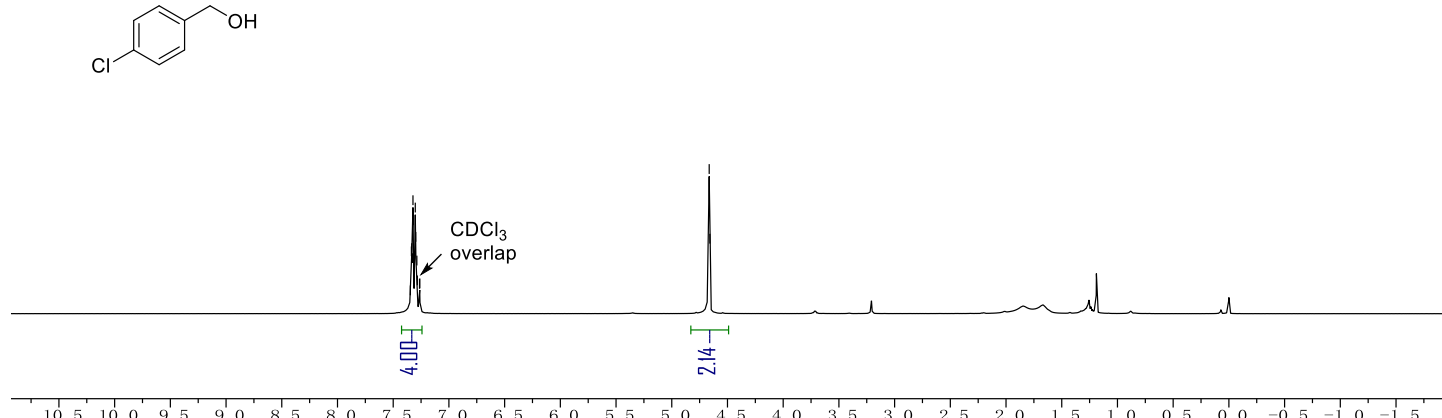

2bc; ${ }^{13} \mathrm{C} \mathrm{NMR,} \mathrm{CDCl}_{3} ; 151 \mathrm{MHz}$

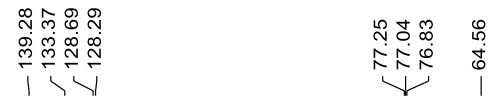

$\mathrm{OOH}_{\mathrm{OH}}$

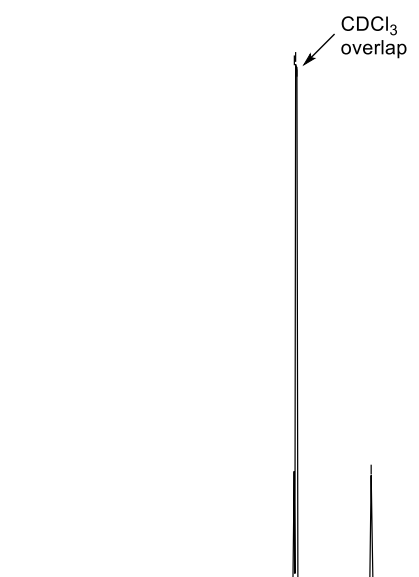

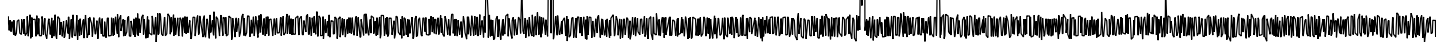

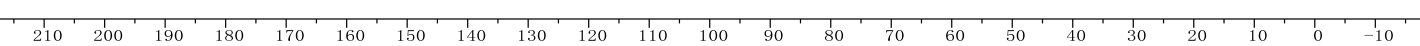

2bd; ${ }^{1} \mathrm{H} \mathrm{NMR} ; \mathrm{CDCl}_{3} ; 400 \mathrm{MHz}$ 


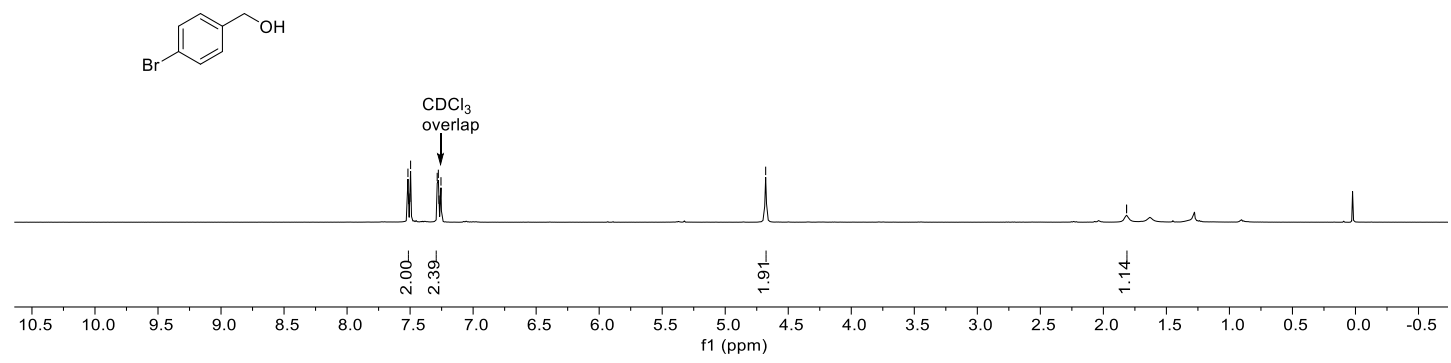

2bd; ${ }^{13} \mathrm{C} \mathrm{NMR} ; \mathrm{CDCl}_{3} ; 101 \mathrm{MHz}$
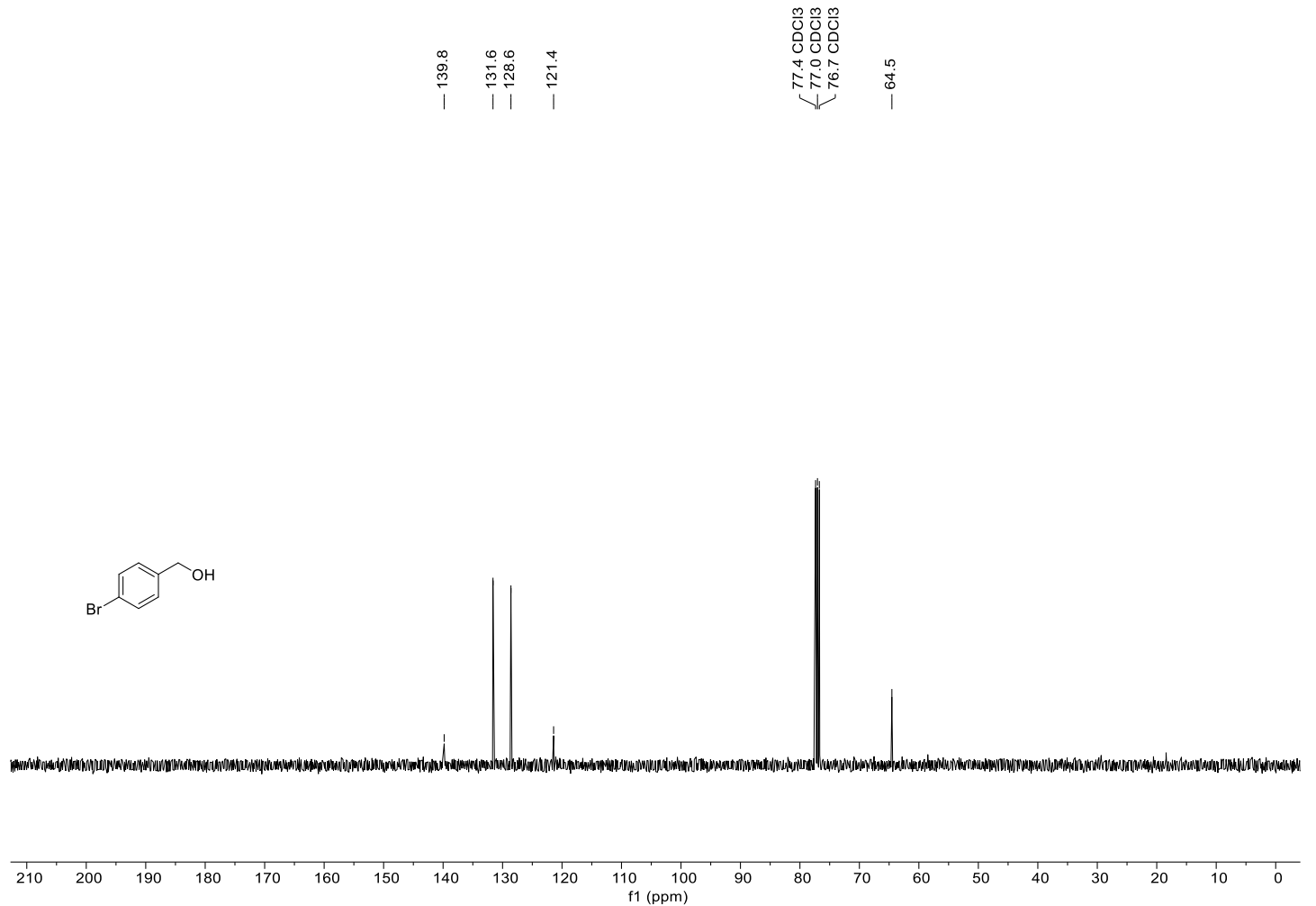

2be; ${ }^{1} \mathrm{H}$ NMR; $\mathrm{CDCl}_{3} ; 400 \mathrm{MHz}$ 


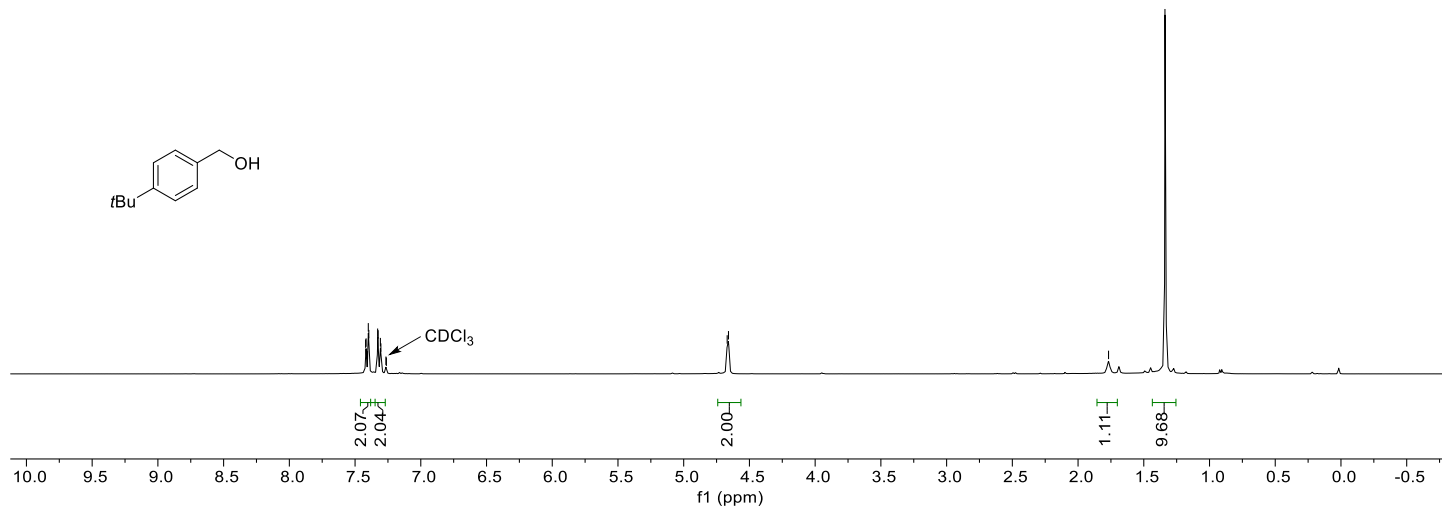

2be; ${ }^{13} \mathrm{C}$ NMR; $\mathrm{CDCl}_{3} ; 101 \mathrm{MHz}$

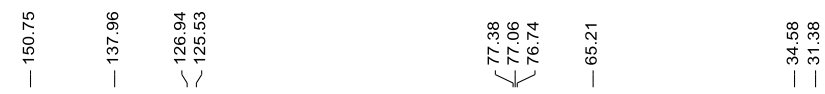

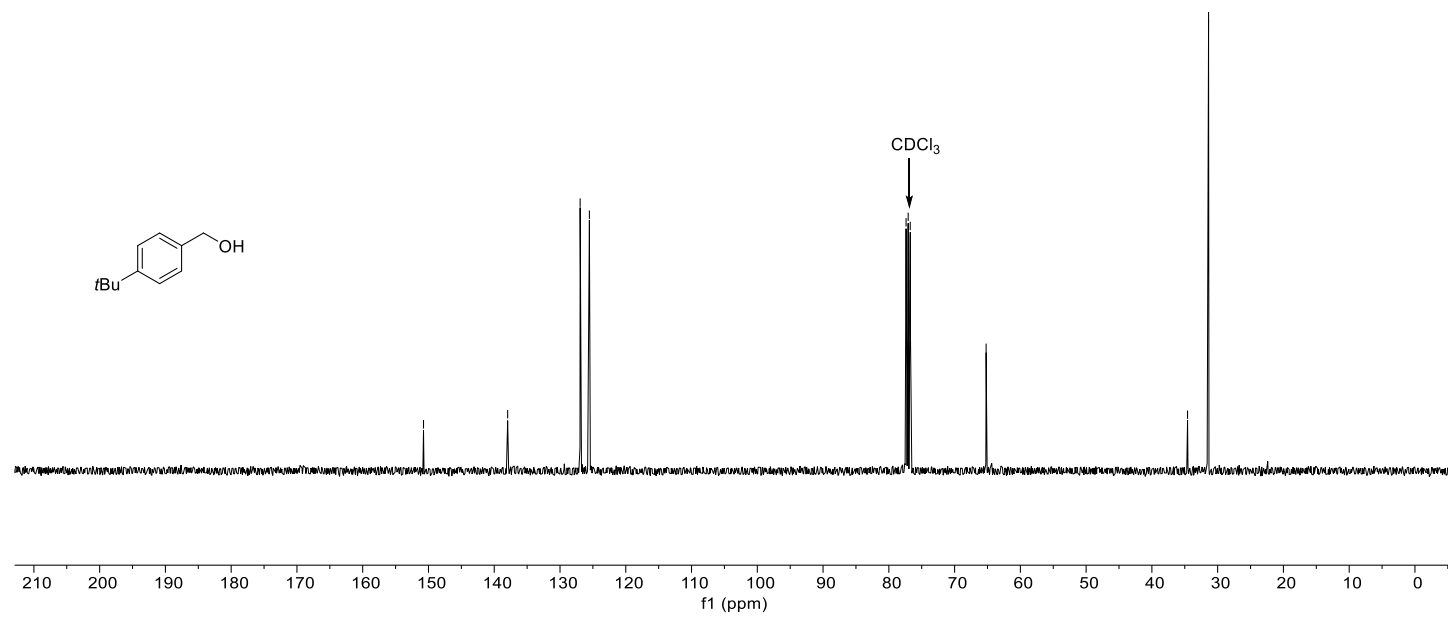

2bf; ${ }^{1} \mathrm{H} \mathrm{NMR} ; \mathrm{CDCl}_{3} ; 400 \mathrm{MHz}$ 


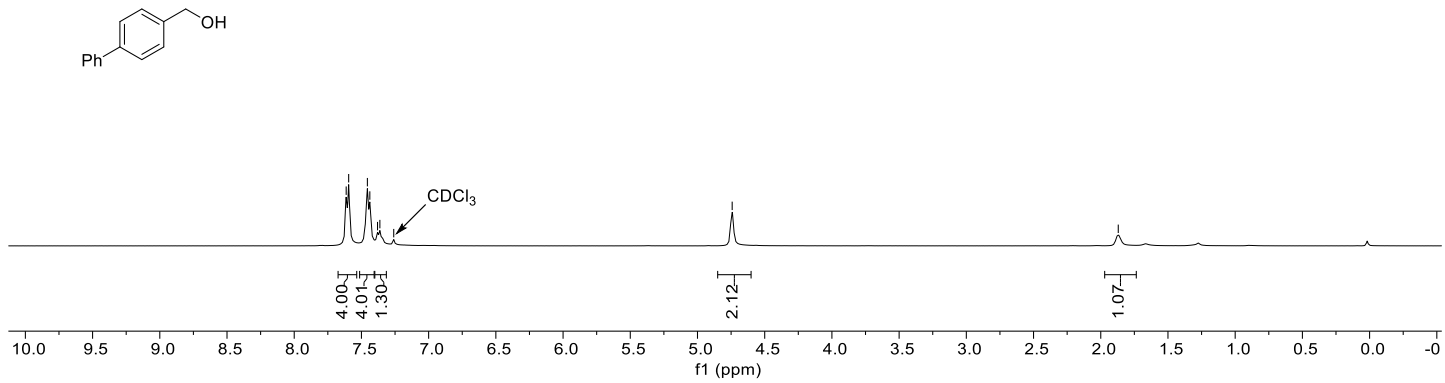

2bf; ${ }^{13} \mathrm{C}$ NMR; $\mathrm{CDCl}_{3} ; 101 \mathrm{MHz}$

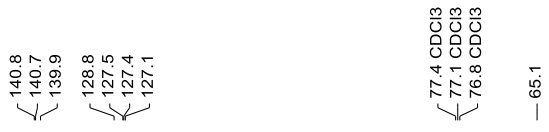
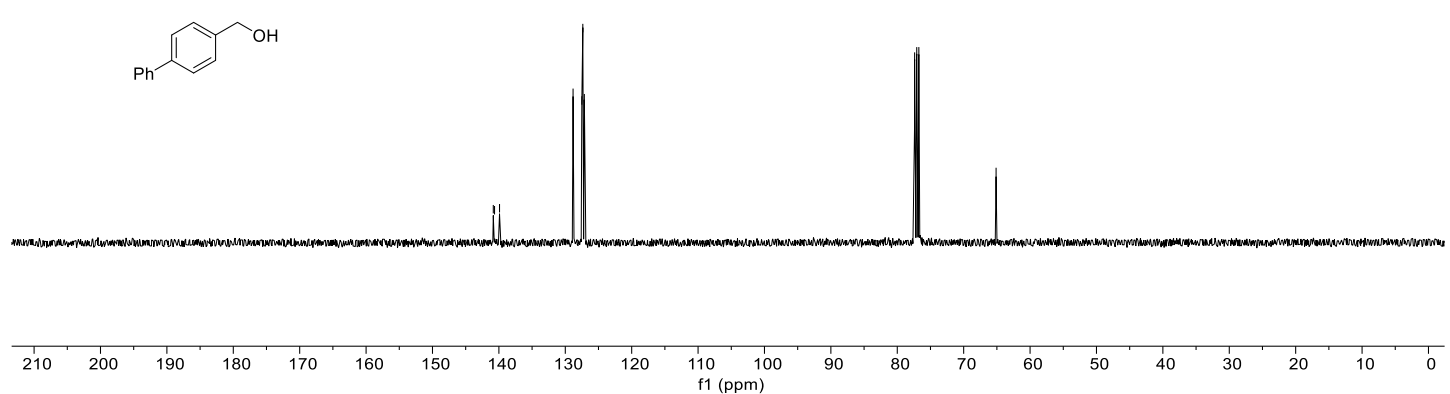

2bg; ${ }^{1} \mathrm{H} \mathrm{NMR} ; \mathrm{CDCl}_{3} ; 400 \mathrm{MHz}$ 


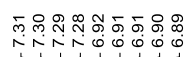

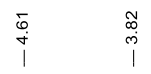

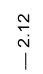

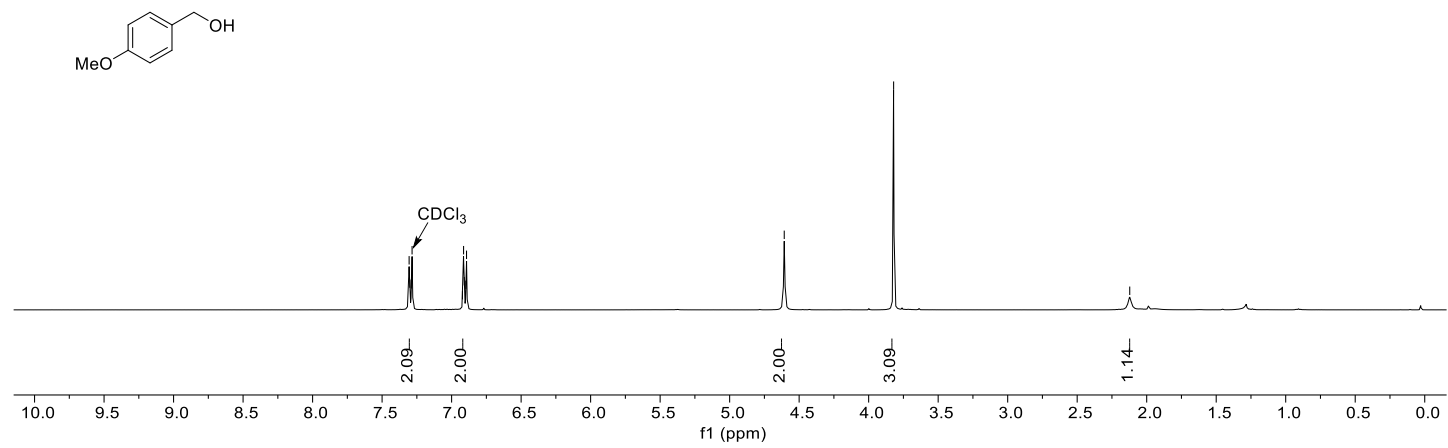

2bg; ${ }^{13} \mathrm{C} \mathrm{NMR} ; \mathrm{CDCl}_{3}, 101 \mathrm{MHz}$

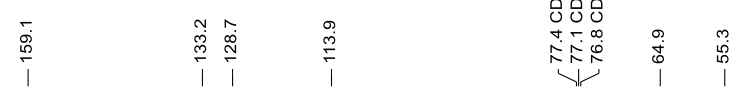
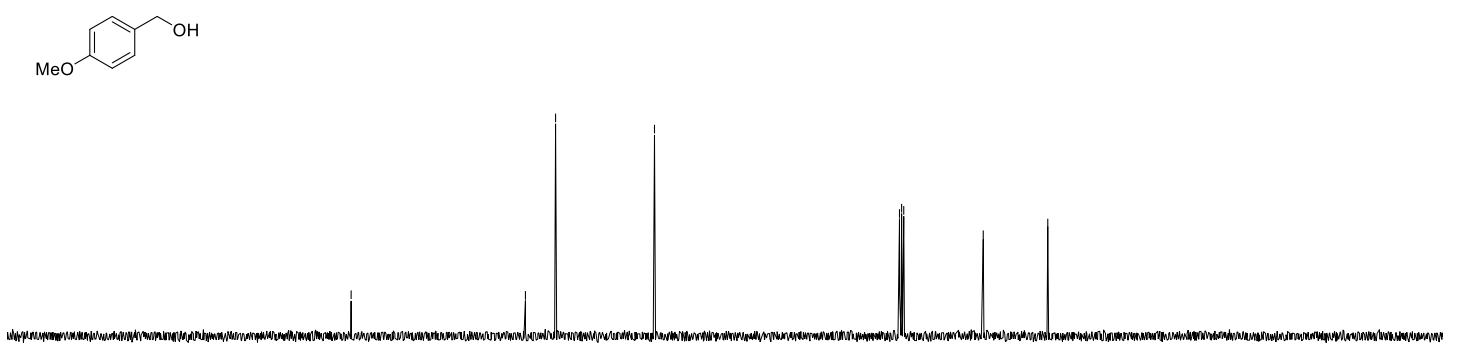

10

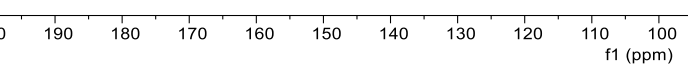

1
90
$\quad 70$

2bh; ${ }^{1} \mathrm{H} \mathrm{NMR} ; \mathrm{CDCl}_{3} ; 400 \mathrm{MHz}$ 
$\overbrace{\mathrm{NCH}}^{\mathrm{OH}}$

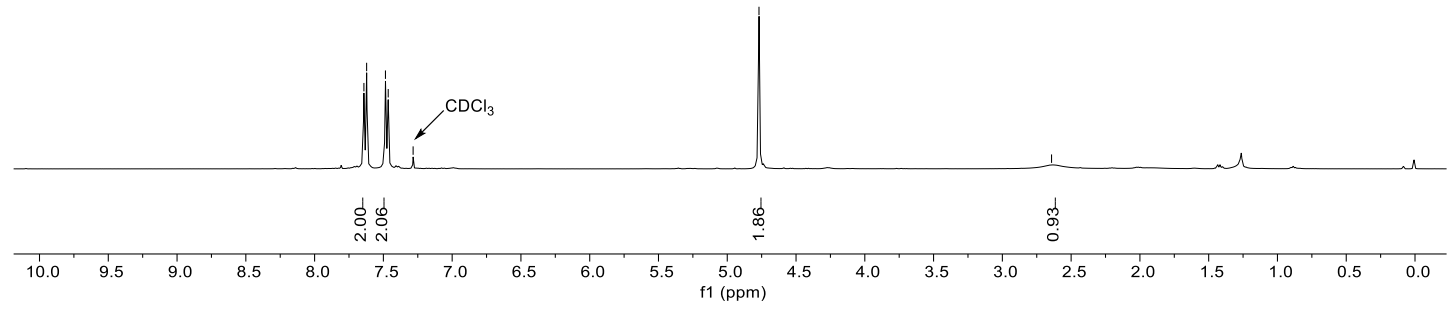

2bh $;{ }^{13} \mathrm{C}$ NMR; $\mathrm{CDCl}_{3} ; 101 \mathrm{MHz}$

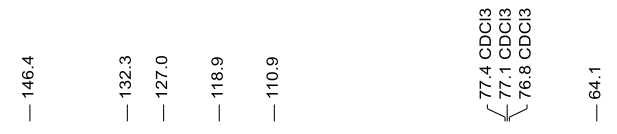
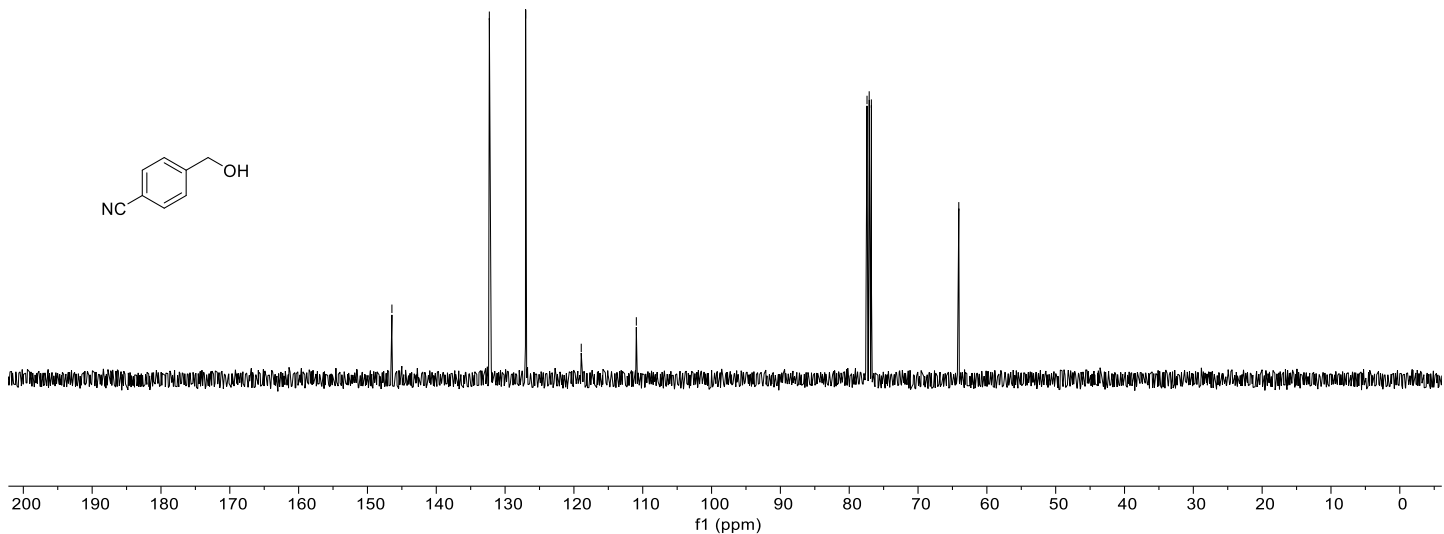

2bi; ${ }^{1} \mathrm{H}$ NMR; $\mathrm{CDCl}_{3} ; 400 \mathrm{MHz}$ 


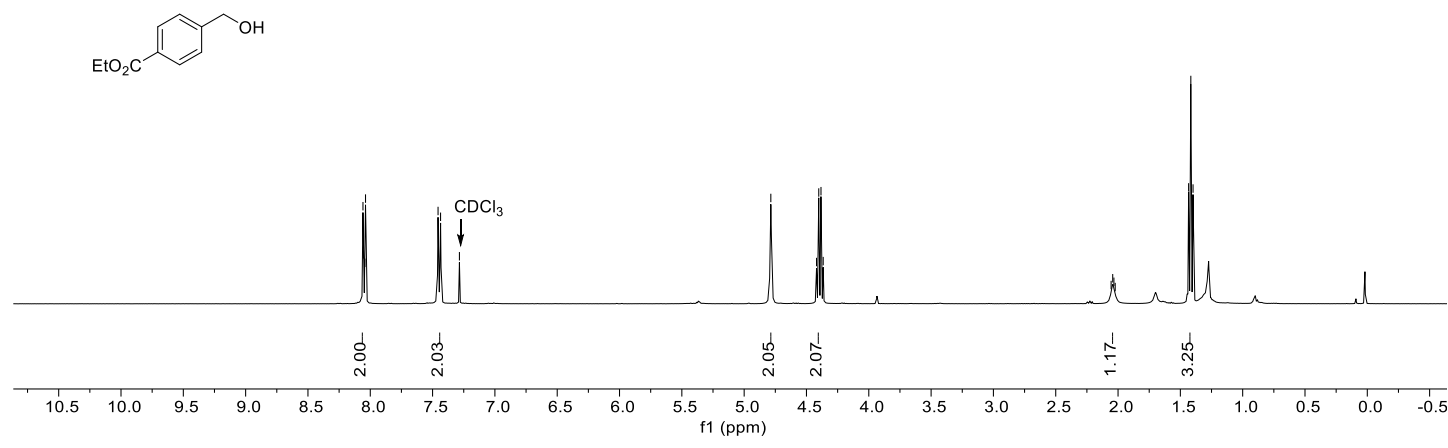

2bi; ${ }^{13} \mathrm{C} \mathrm{NMR} ; \mathrm{CDCl}_{3} ; 101 \mathrm{MHz}$
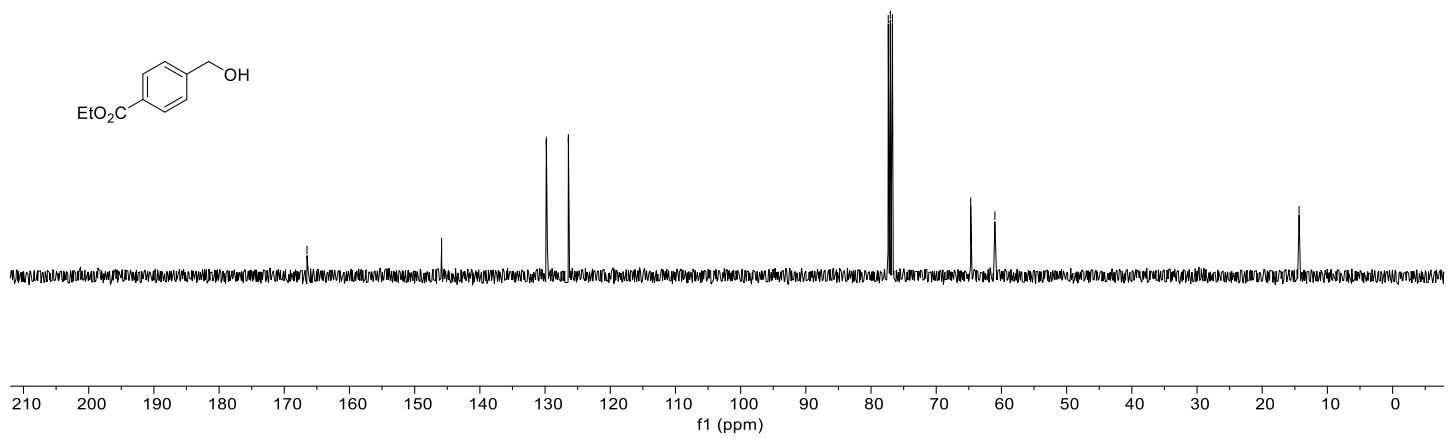

2bj; ${ }^{1} \mathrm{H} \mathrm{NMR} ; \mathrm{CDCl}_{3} ; 400 \mathrm{MHz}$ 


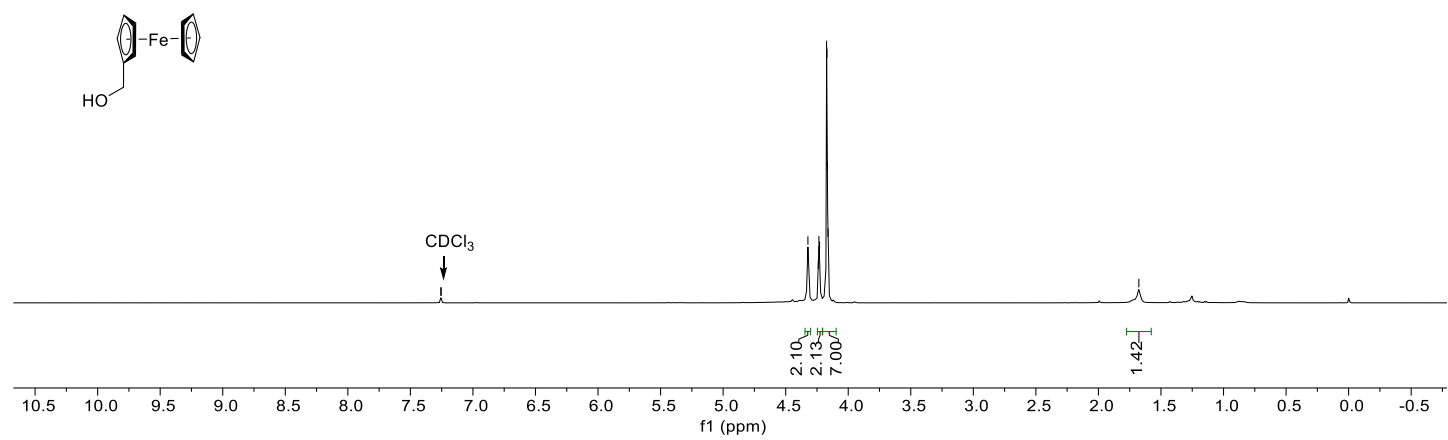

2bj; ${ }^{13} \mathrm{C} \mathrm{NMR;} \mathrm{CDCl}_{3} ; 101 \mathrm{MHz}$

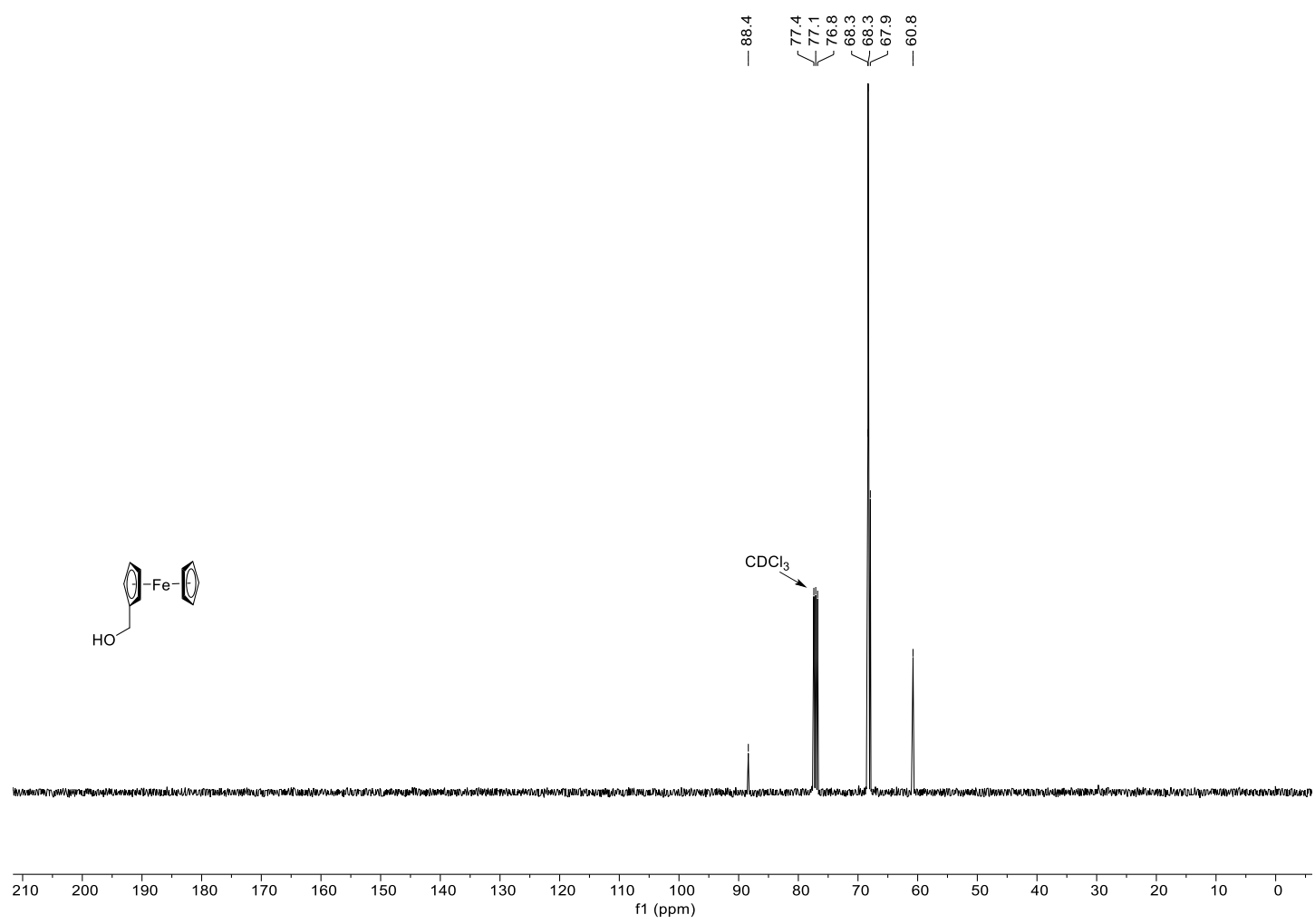

2bk; ${ }^{1} \mathrm{H} \mathrm{NMR}, \mathrm{CDCl}_{3} ; 400 \mathrm{MHz}$ 


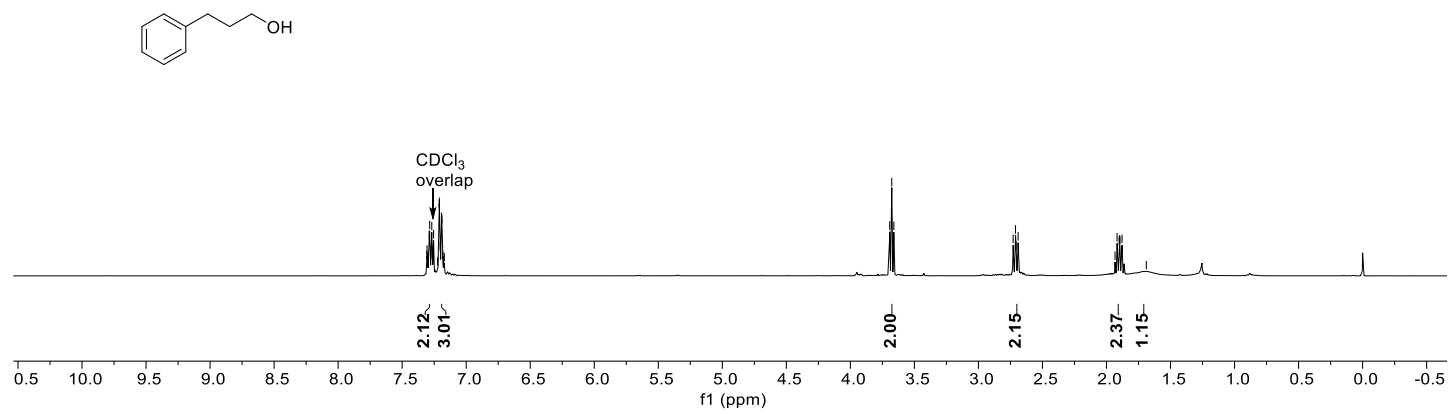

2bk; ${ }^{13} \mathrm{C} \mathrm{NMR,} \mathrm{CDCl}_{3} ; 101 \mathrm{MHz}$

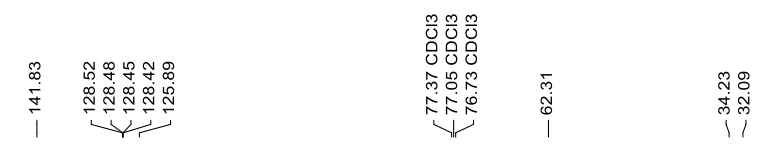
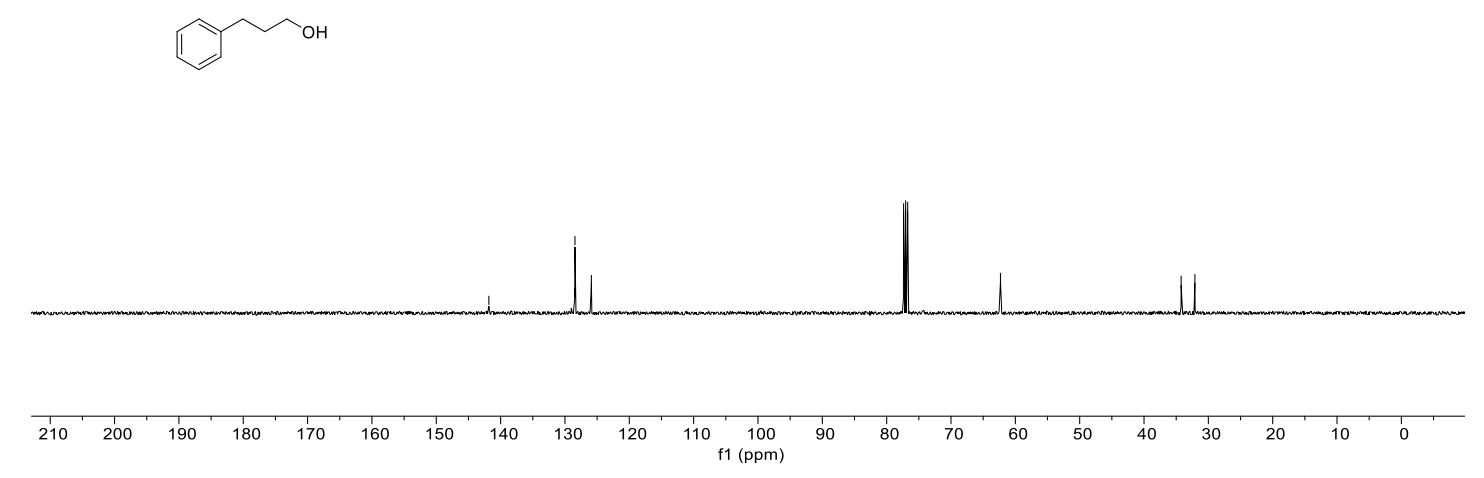

2bl; ${ }^{1} \mathrm{H} \mathrm{NMR} ; \mathrm{CDCl}_{3} ; 400 \mathrm{MHz}$ 


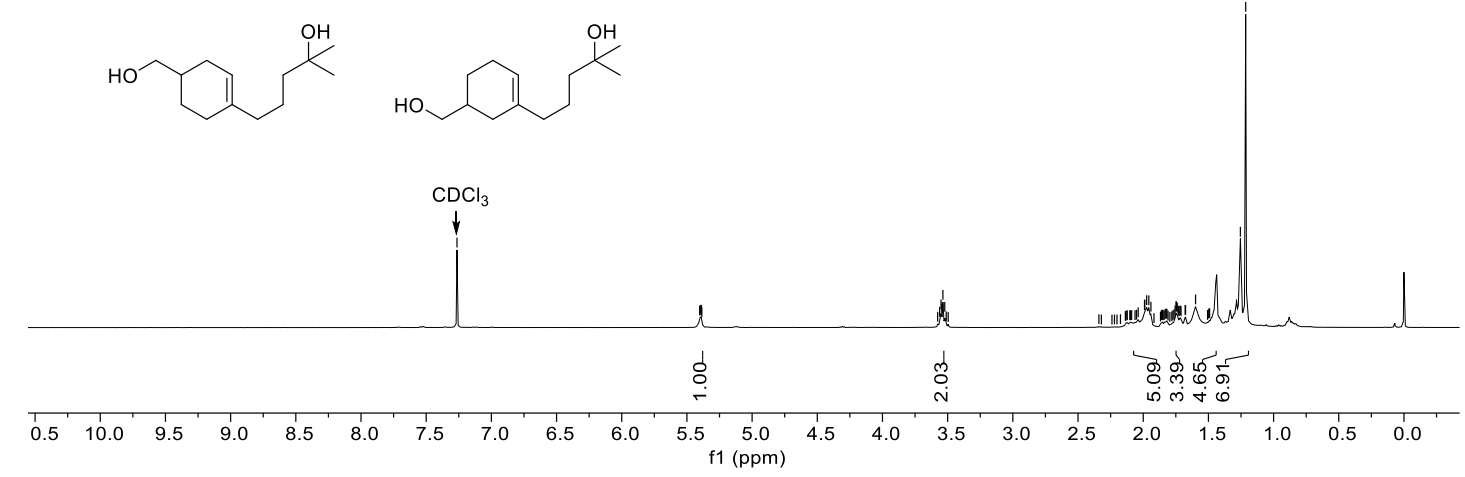

2bl; ${ }^{13} \mathrm{C}$ NMR; $\mathrm{CDCl}_{3} ; 101 \mathrm{MHz}$
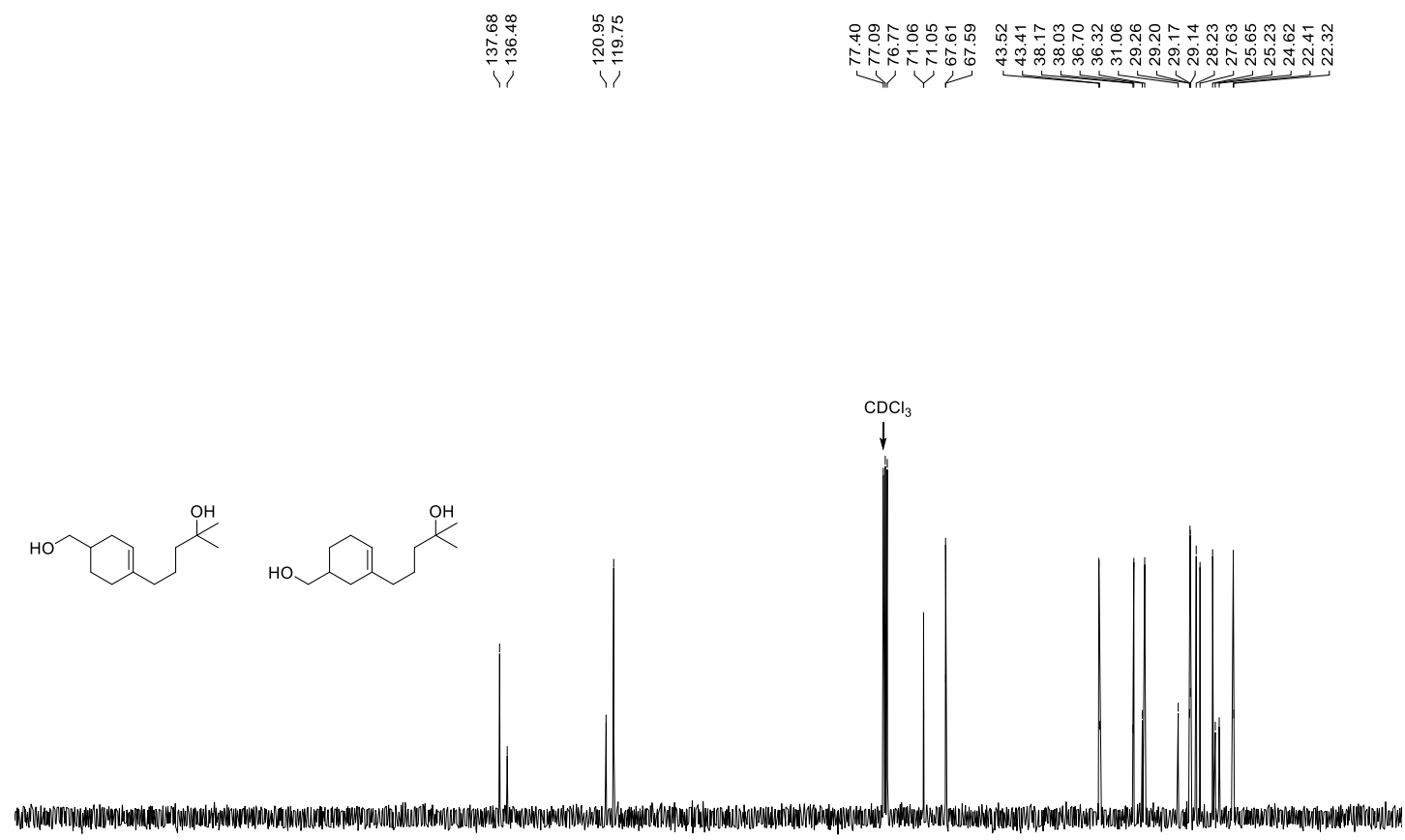

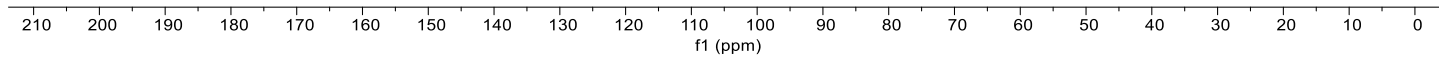

2bm; ${ }^{1} \mathrm{H} \mathrm{NMR} ; \mathrm{CDCl}_{3} ; 400 \mathrm{MHz}$ 


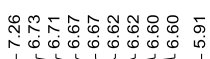

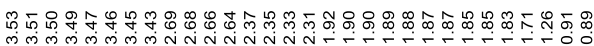

1

管

ON $\mathrm{CH}_{3} \mathrm{OH}$

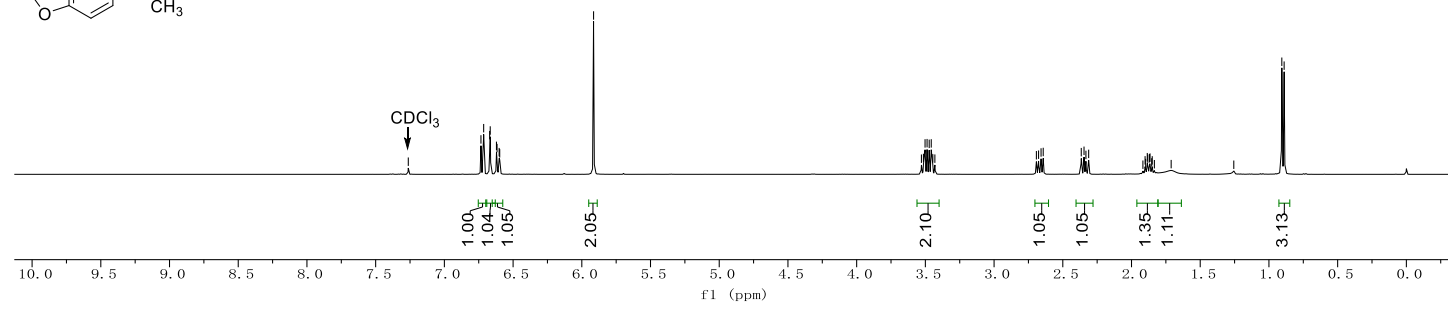

2bm; ${ }^{13} \mathrm{C} \mathrm{NMR} ; \mathrm{CDCl}_{3} ; 101 \mathrm{MHz}$

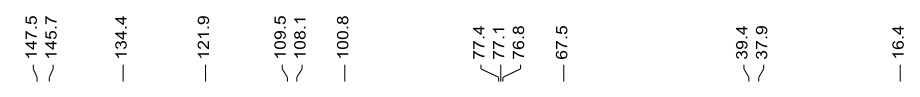

$\mathrm{CH}_{\mathrm{OH}}^{\mathrm{OH}}$

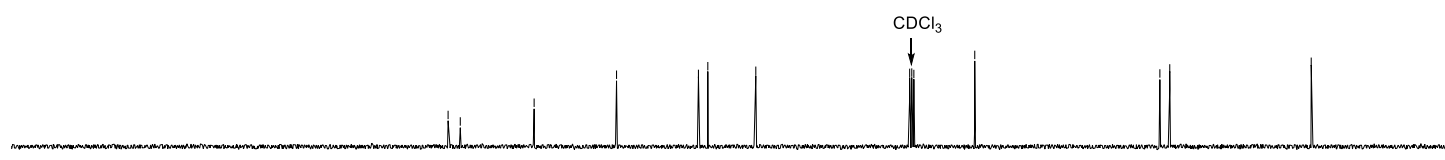

\begin{tabular}{lllllllllllllllllllll}
\hline 210 & 200 & 190 & 180 & 170 & 160 & 150 & 140 & 130 & 120 & $\begin{array}{c}110 \\
\mathrm{f} 1(\mathrm{ppm})\end{array}$ & 90 & 80 & 70 & 60 & 50 & 40 & 30 & 20 & 10 & 0
\end{tabular}

2bn; ${ }^{1} \mathrm{H} \mathrm{NMR} ; \mathrm{CDCl}_{3} ; 400 \mathrm{MHz}$ 


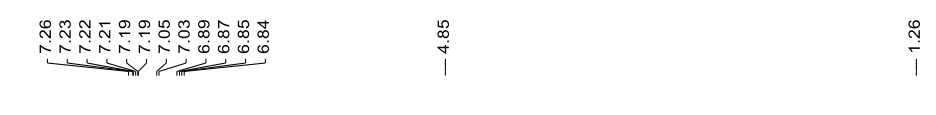

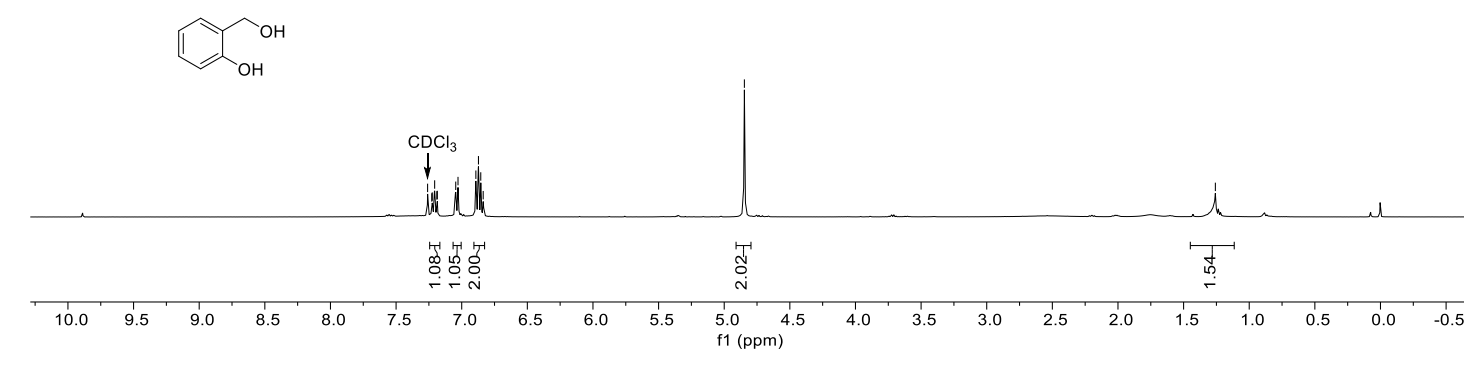

2bn; ${ }^{13} \mathrm{C} \mathrm{NMR} ; \mathrm{CDCl}_{3} ; 101 \mathrm{MHz}$

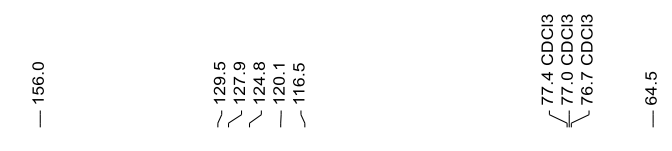

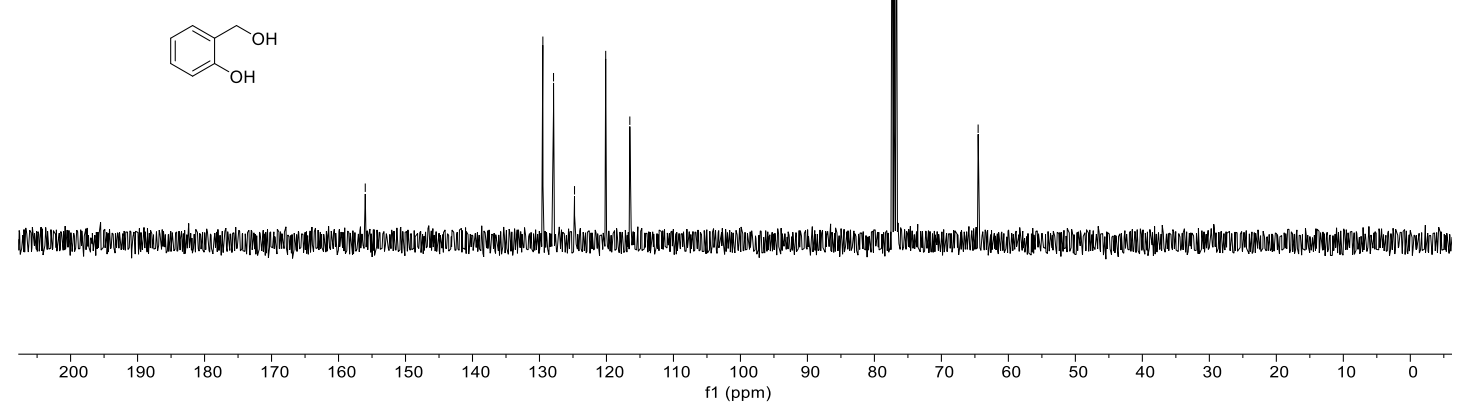

2bo; ${ }^{1} \mathrm{H} \mathrm{NMR} ; \mathrm{CDCl}_{3} ; 400 \mathrm{MHz}$ 


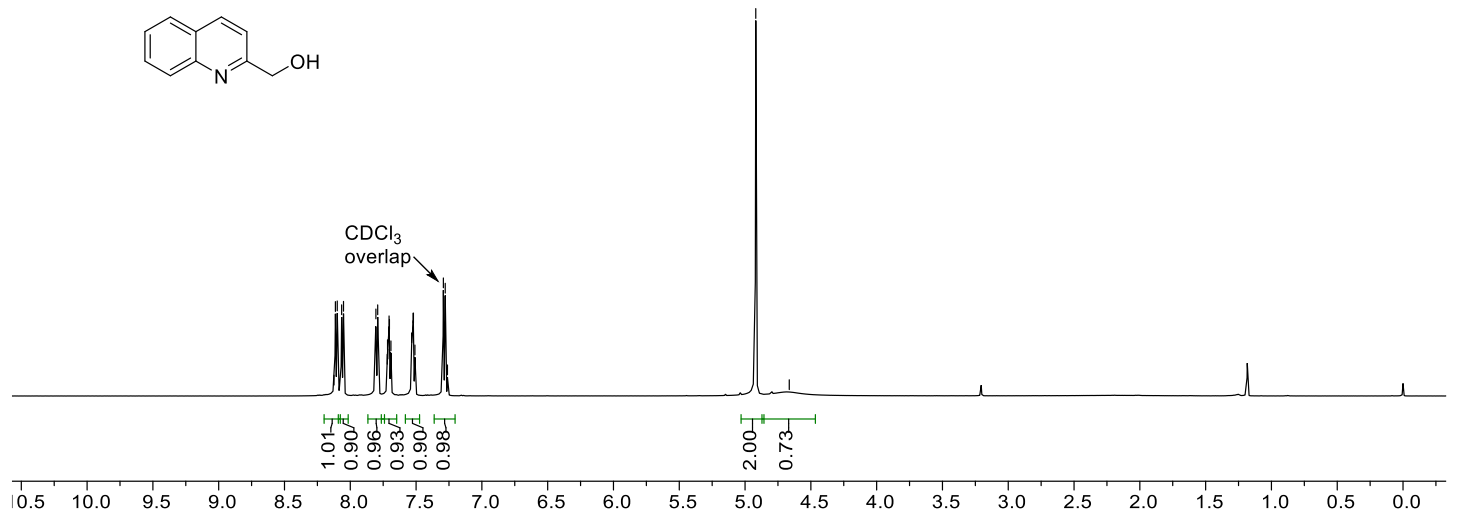

2bo; ${ }^{13} \mathrm{C} \mathrm{NMR} ; \mathrm{CDCl}_{3} ; 101 \mathrm{MHz}$

l

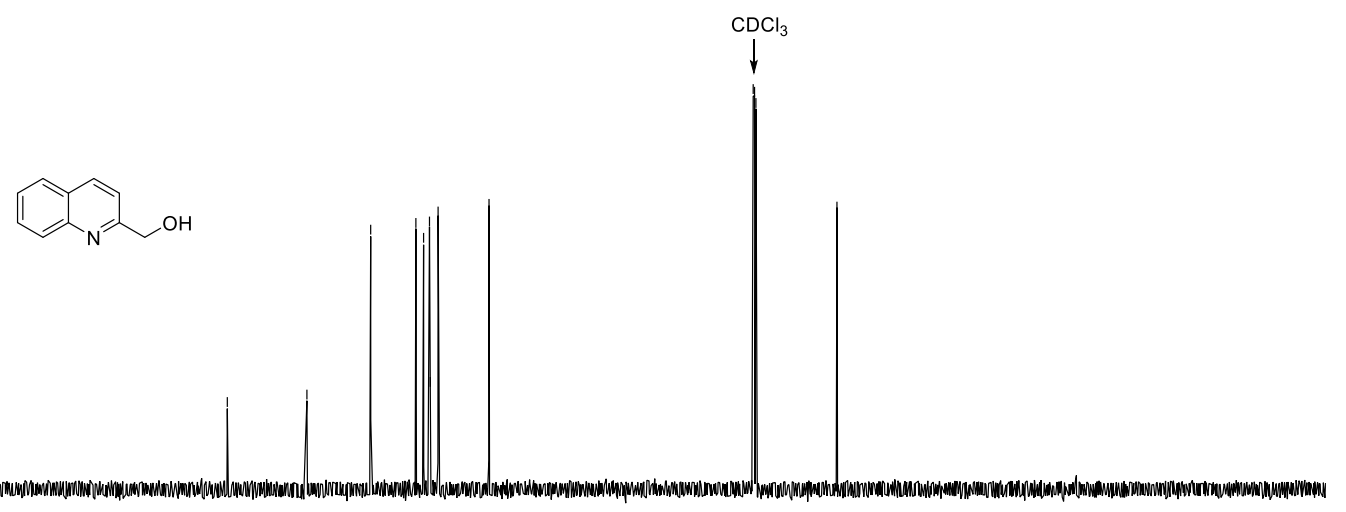

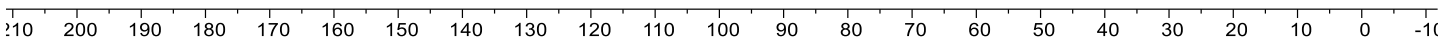

2bp; ${ }^{1} \mathrm{H} \mathrm{NMR} ; \mathrm{CDCl}_{3} ; 400 \mathrm{MHz}$ 


\begin{tabular}{|c|}
\hline 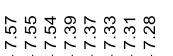 \\
\hline
\end{tabular}

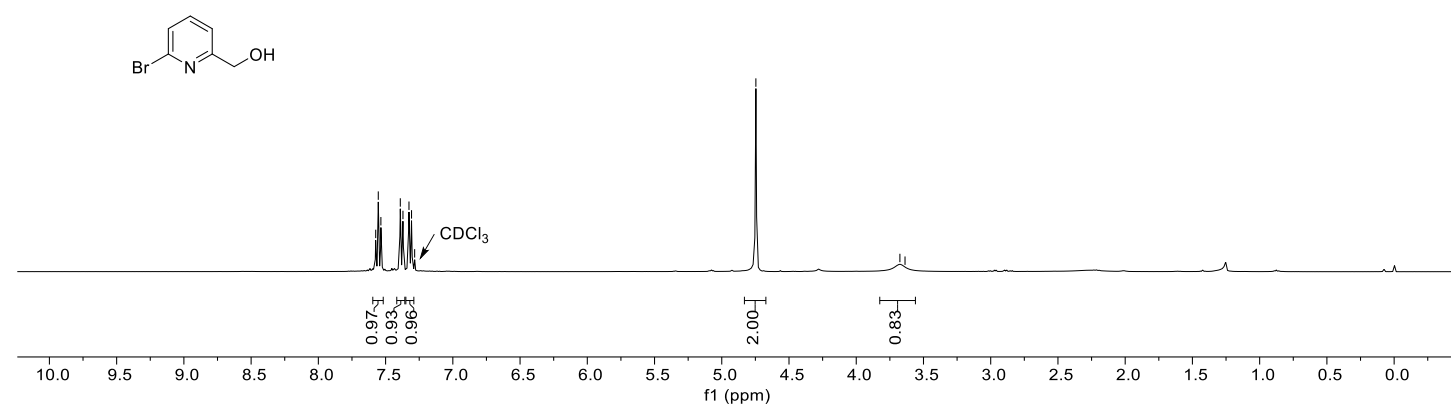

2bp; ${ }^{13} \mathrm{C} \mathrm{NMR} ; \mathrm{CDCl}_{3} ; 101 \mathrm{MHz}$

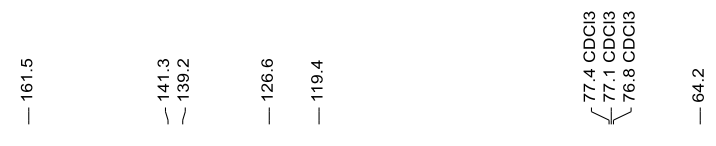

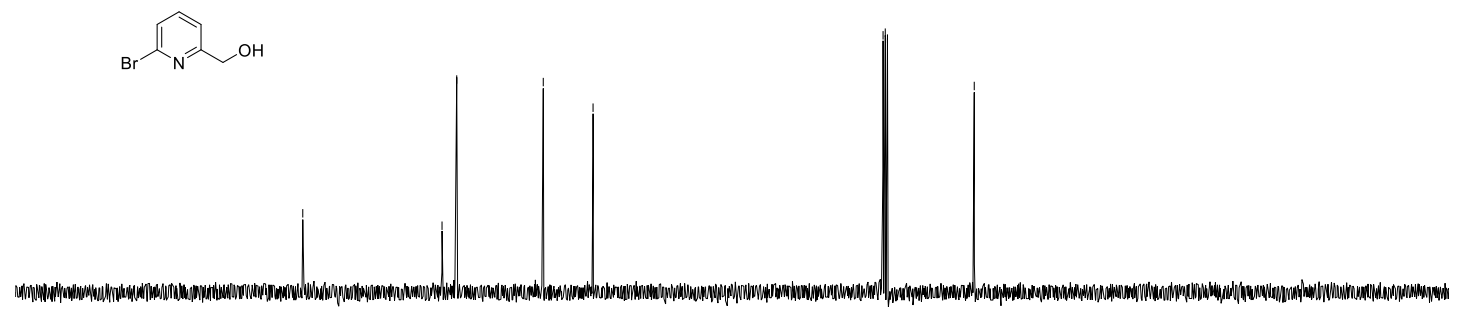

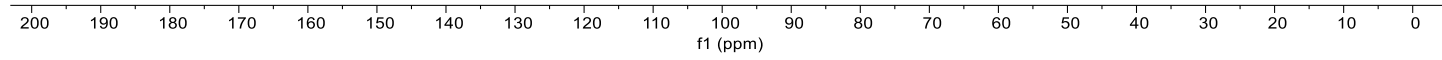

2bq; ${ }^{1} \mathrm{H}$ NMR; $\mathrm{CDCl}_{3} ; 400 \mathrm{MHz}$ 


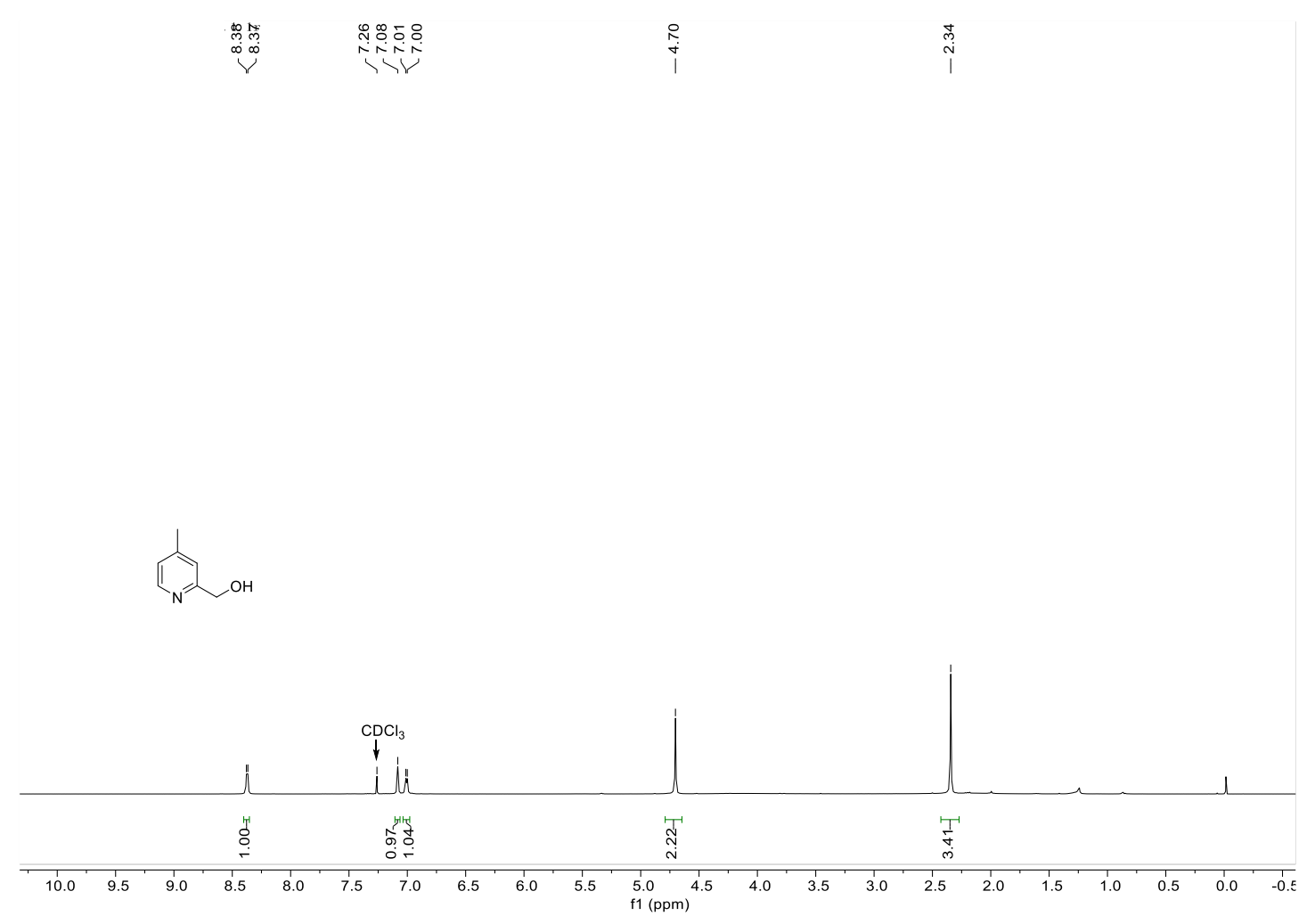

2bq; ${ }^{13} \mathrm{C} \mathrm{NMR} ; \mathrm{CDCl}_{3} ; 101 \mathrm{MHz}$

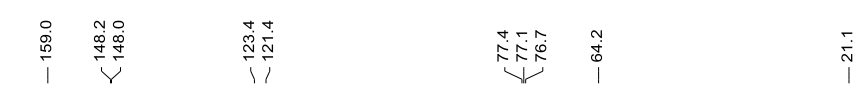

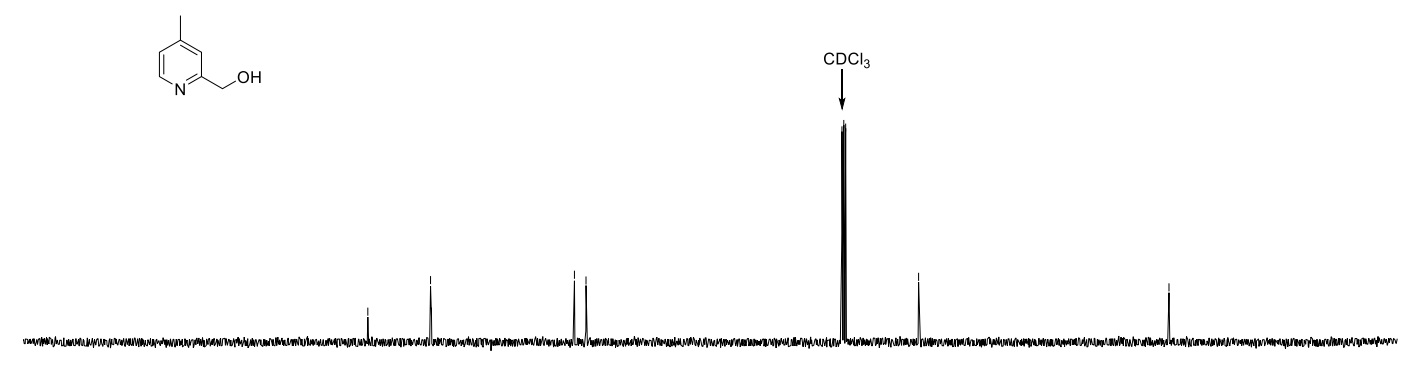

2br; ${ }^{1} \mathrm{H} \mathrm{NMR} ; \mathrm{CDCl}_{3} ; 400 \mathrm{MHz}$ 


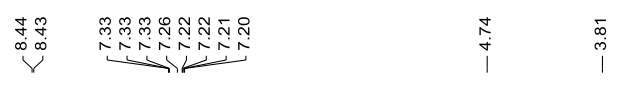

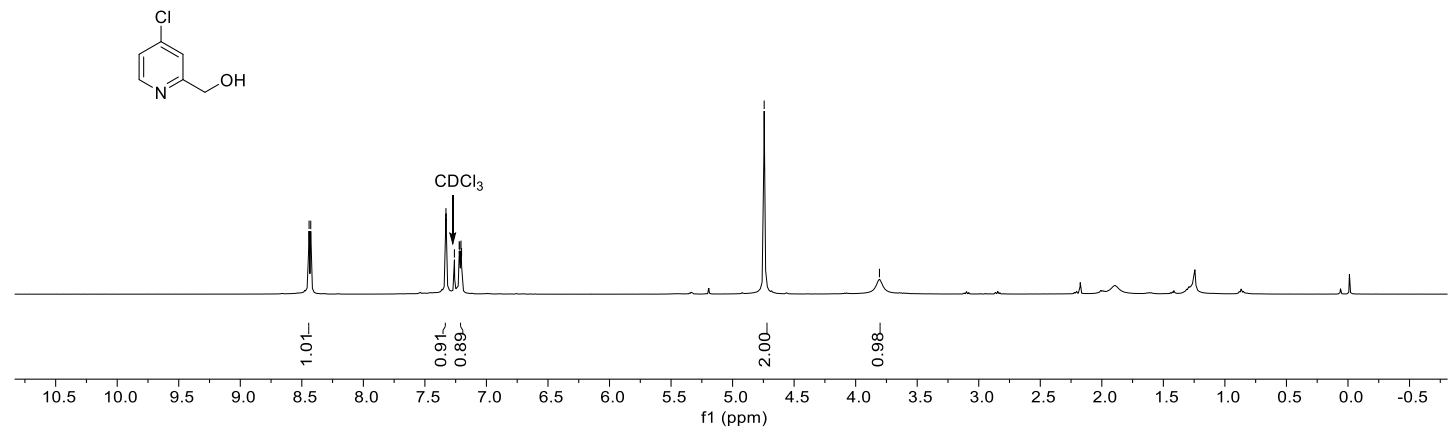

2br; ${ }^{13} \mathrm{C} \mathrm{NMR} ; \mathrm{CDCl}_{3} ; 101 \mathrm{MHz}$

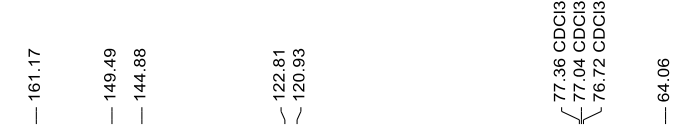
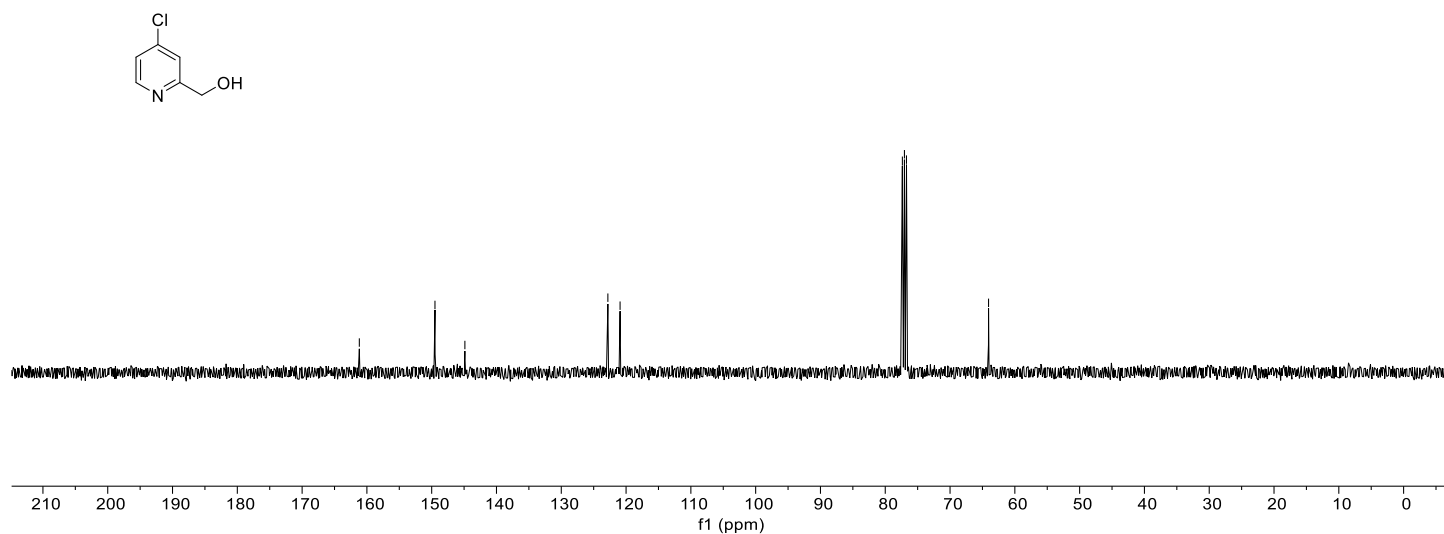

2bs; ${ }^{1} \mathrm{H} \mathrm{NMR} ; \mathrm{CDCl}_{3} ; 400 \mathrm{MHz}$ 


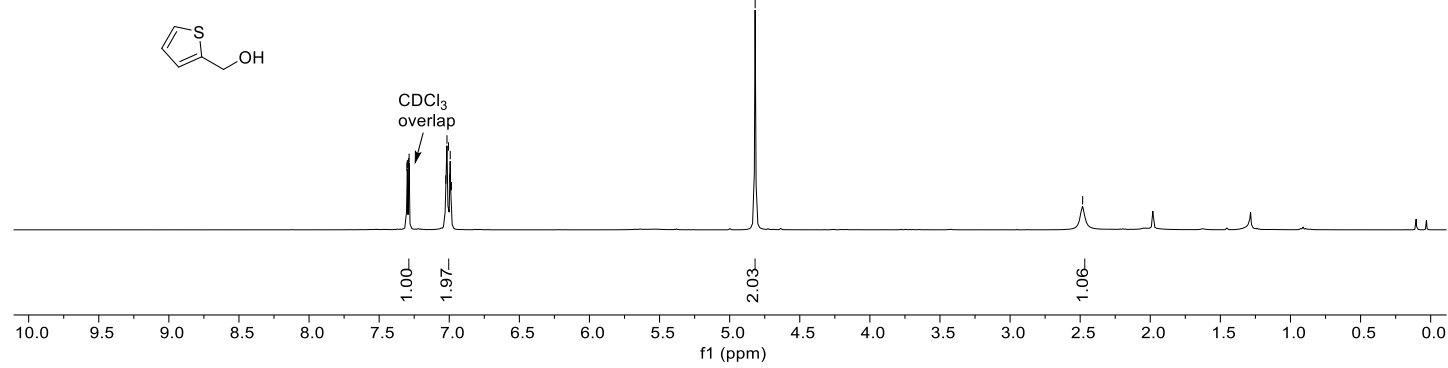

2bs; ${ }^{13} \mathrm{C} \mathrm{NMR} ; \mathrm{CDCl}_{3} ; 101 \mathrm{MHz}$

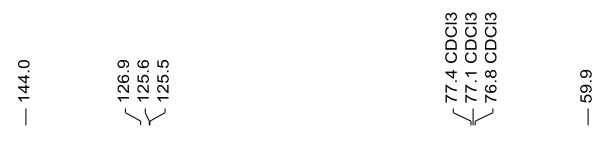
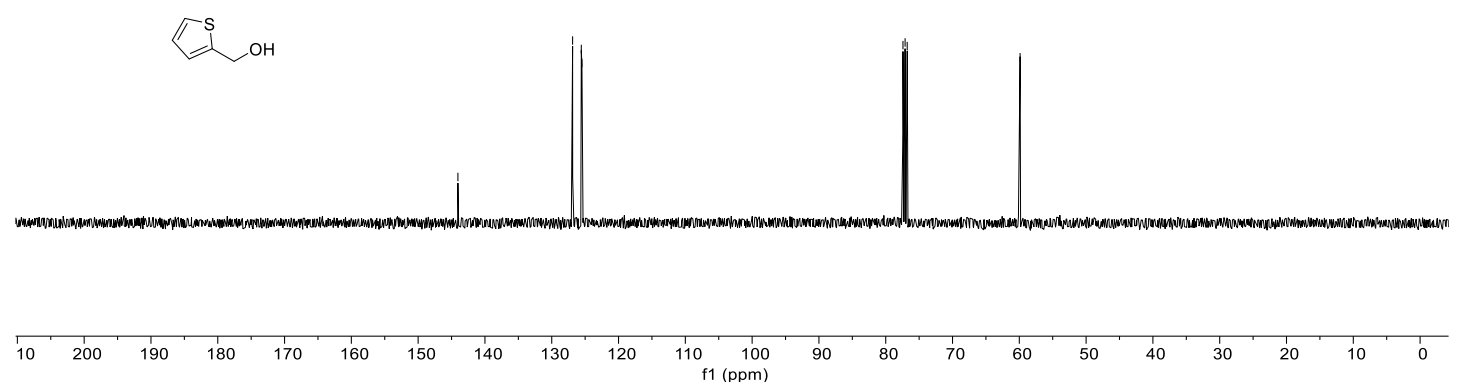

2bt; ${ }^{1} \mathrm{H} \mathrm{NMR} ; \mathrm{CDCl}_{3} ; 400 \mathrm{MHz}$ 


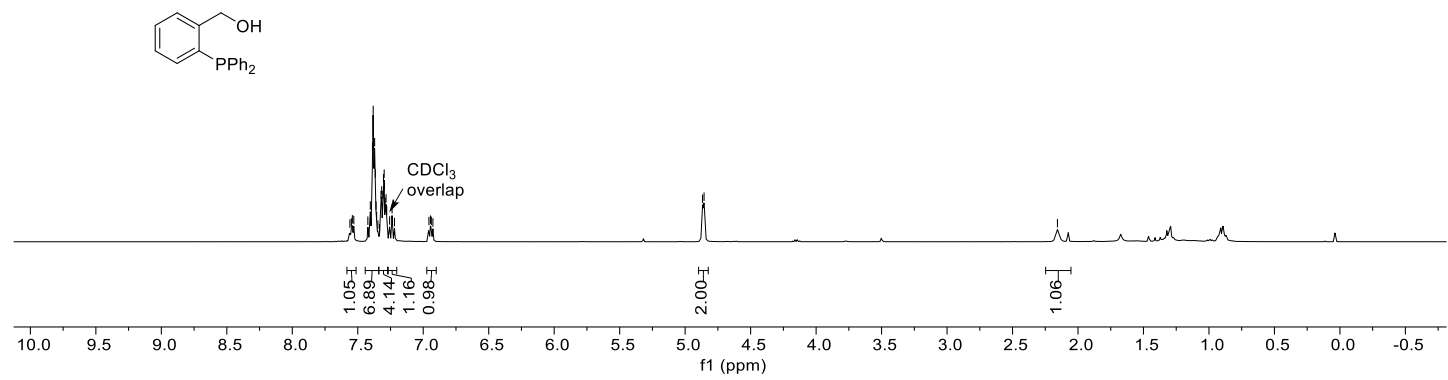

2bt; ${ }^{31} \mathrm{P} \mathrm{NMR} ; \mathrm{CDCl}_{3} ; 162 \mathrm{MHz}$
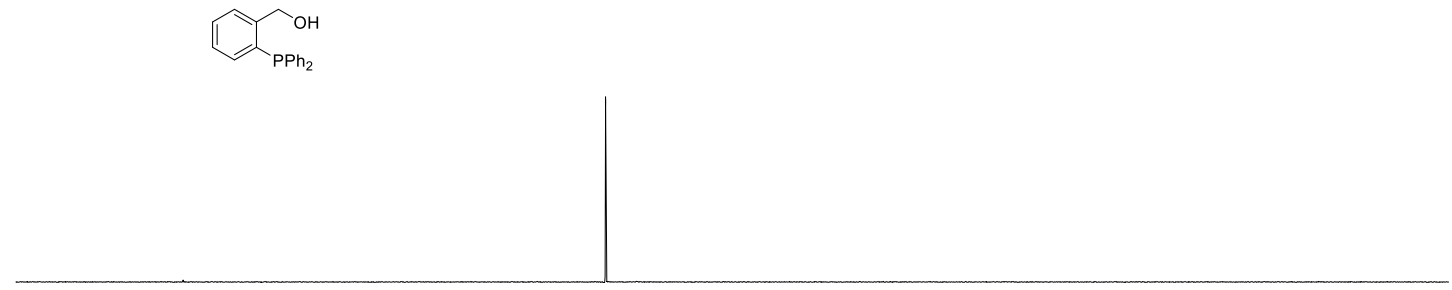

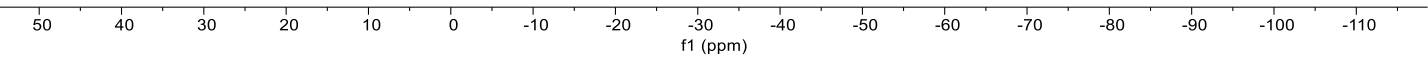

2bt; ${ }^{13} \mathrm{C} \mathrm{NMR} ; \mathrm{CDCl}_{3} ; 101 \mathrm{MHz}$ 


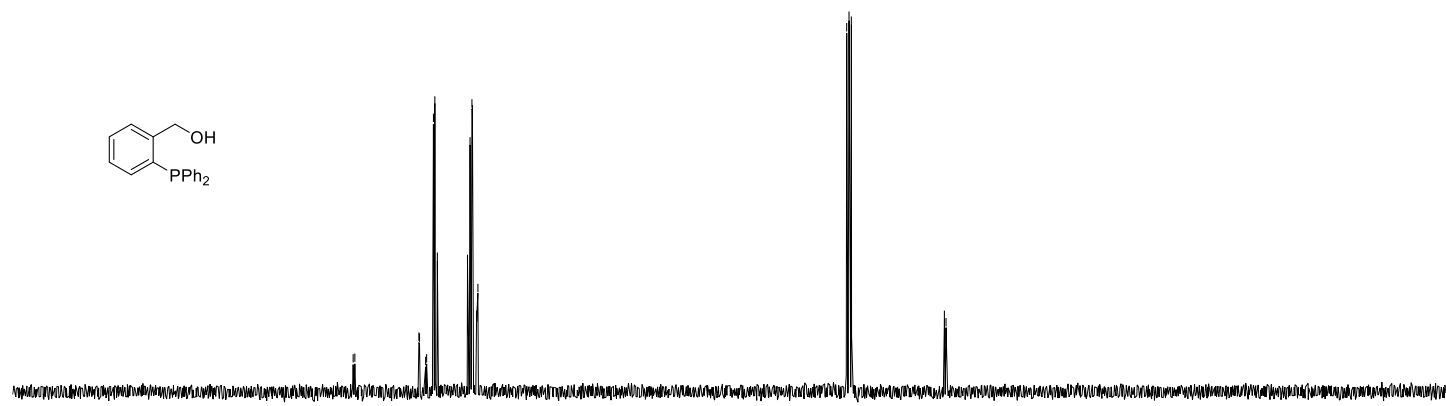

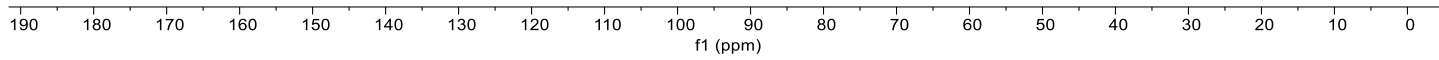

2bu; ${ }^{1} \mathrm{H}$ NMR; $\mathrm{CDCl}_{3} ; 400 \mathrm{MHz}$
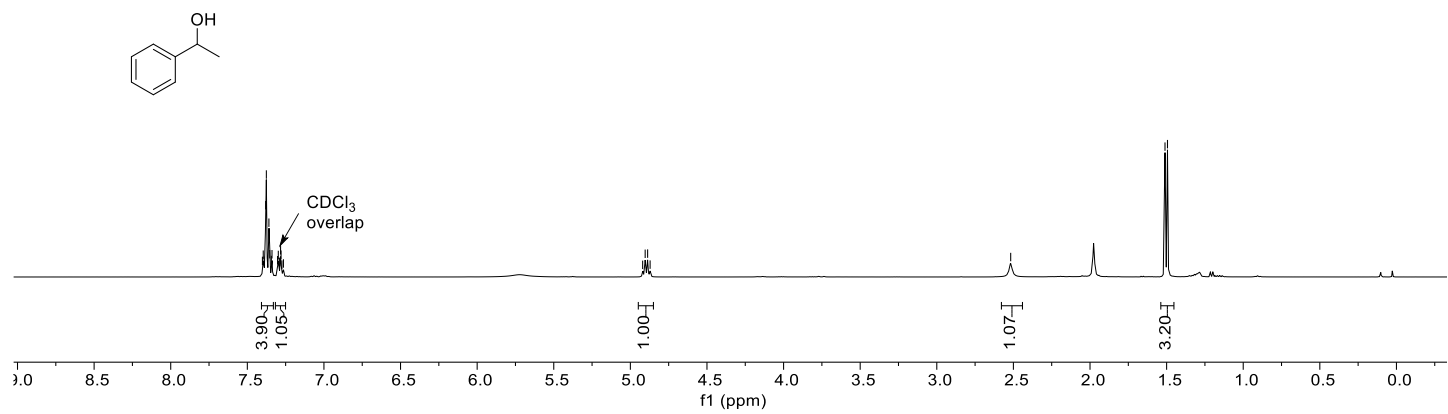

2bu; ${ }^{13} \mathrm{C} \mathrm{NMR} ; \mathrm{CDCl}_{3} ; 101 \mathrm{MHz}$ 


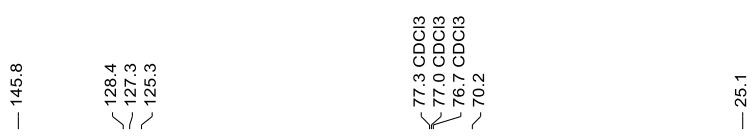

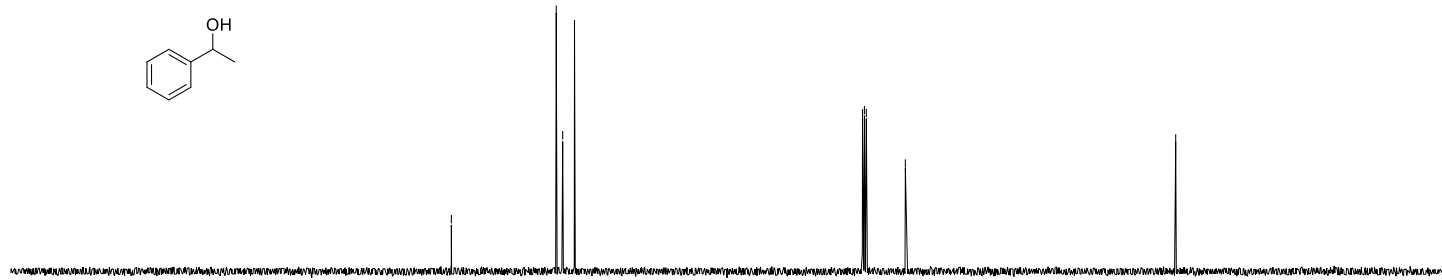

$\begin{array}{llllllllllllllllllllllllll}1 & 1 & 200 & 190 & 180 & 170 & 160 & 150 & 140 & 130 & 120 & 110 & 100 & 10 & 10 & 10 & 60 & 50 & 40 & 30 & 20 & 10 & 0 & -10\end{array}$

2bv; ${ }^{1} \mathrm{H} \mathrm{NMR} ; \mathrm{CDCl}_{3} ; 400 \mathrm{MHz}$

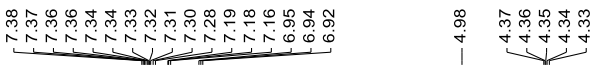

$\overbrace{0}^{\mathrm{N}} \mathrm{N}_{\mathrm{N}}^{\mathrm{H}}$

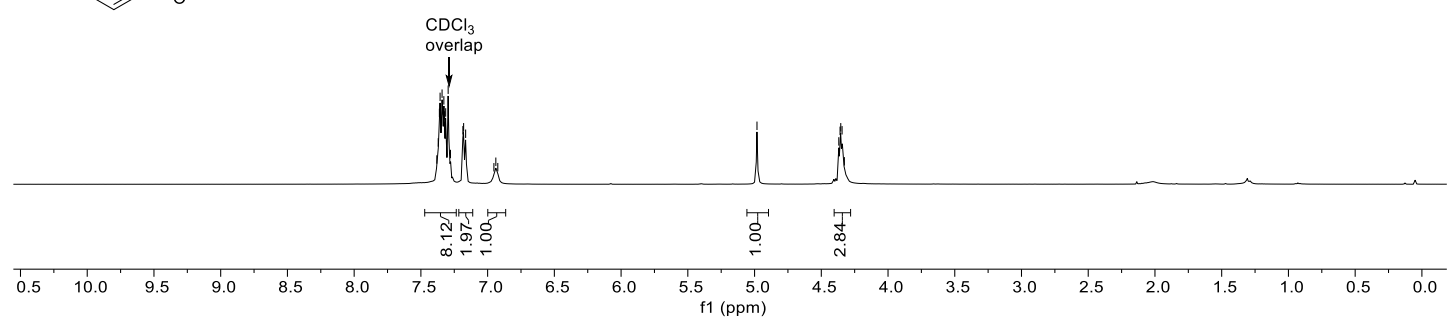

2bv; ${ }^{13} \mathrm{C} \mathrm{NMR} ; \mathrm{CDCl}_{3} ; 101 \mathrm{MHz}$ 


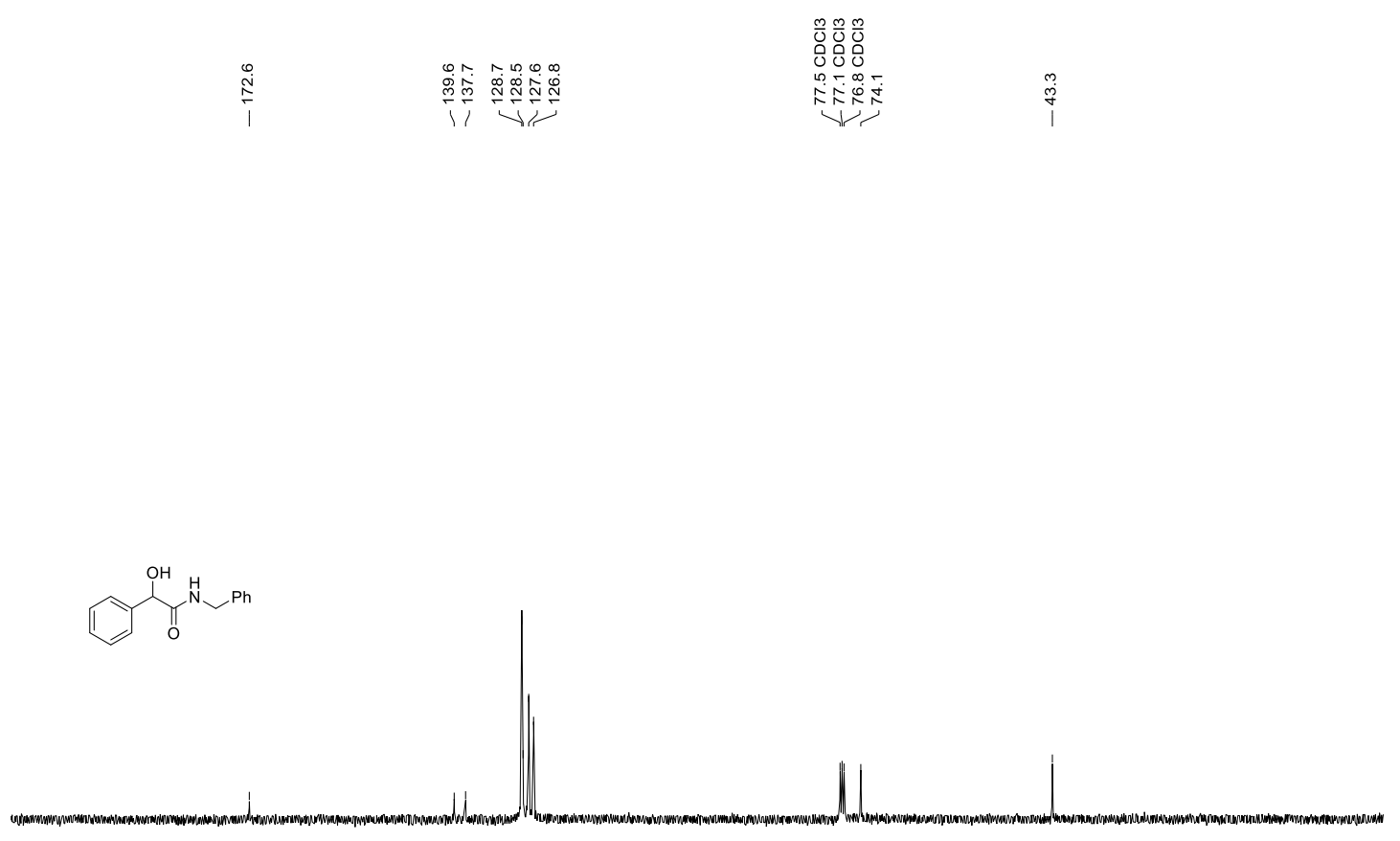

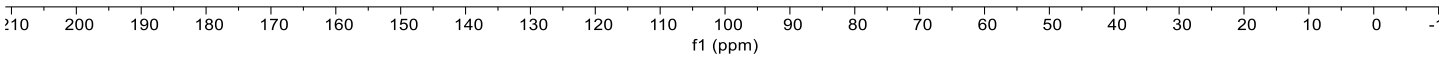

2bw; ${ }^{1} \mathrm{H}$ NMR; $\mathrm{CDCl}_{3} ; 400 \mathrm{MHz}$

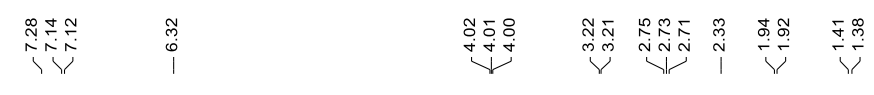

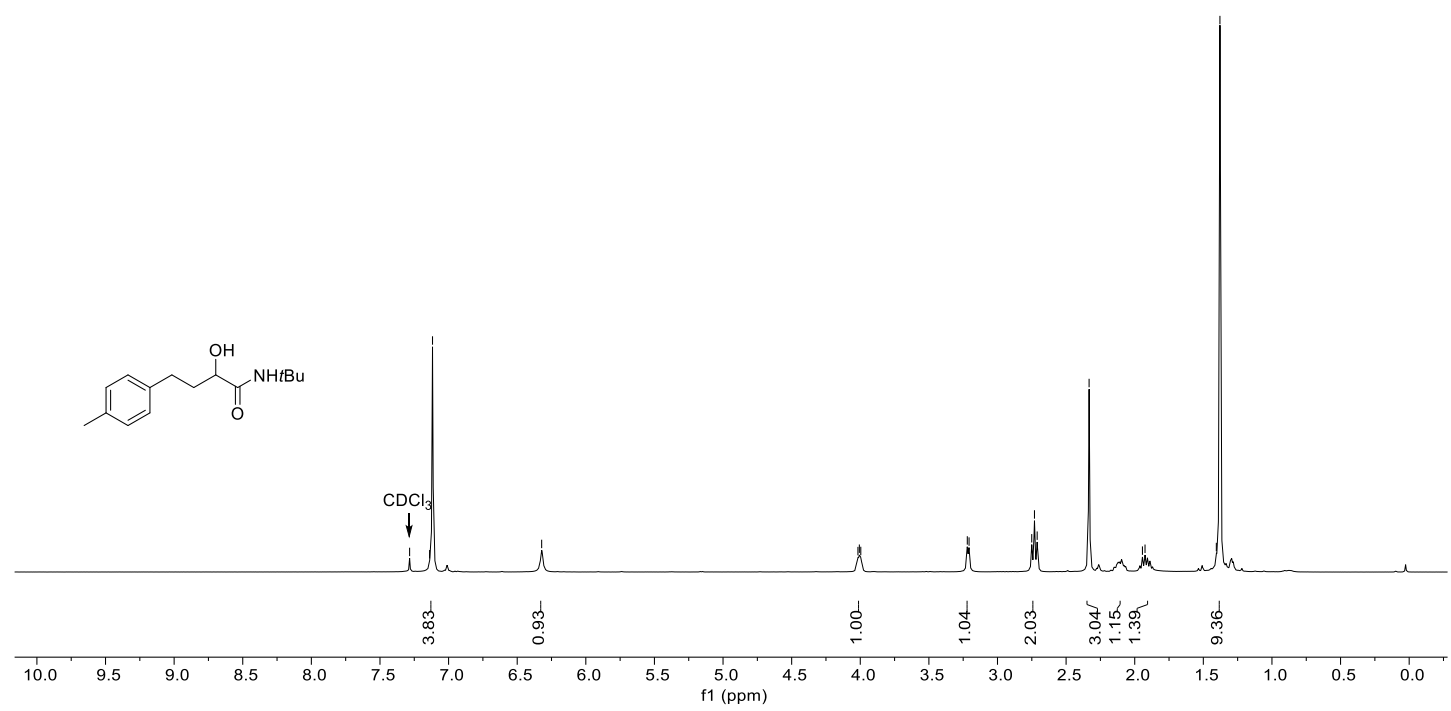

2bw; ${ }^{13} \mathrm{C} \mathrm{NMR} ; \mathrm{CDCl}_{3} ; 101 \mathrm{MHz}$ 


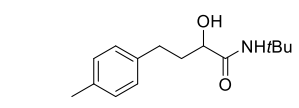

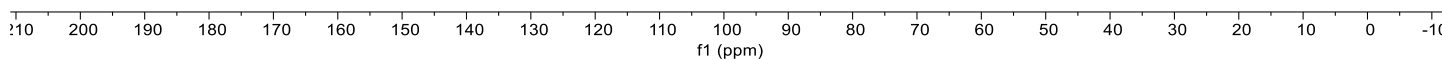

2bx; ${ }^{1} \mathrm{H}$ NMR; $\mathrm{CDCl}_{3} ; 400 \mathrm{MHz}$

$\underbrace{\circ}$

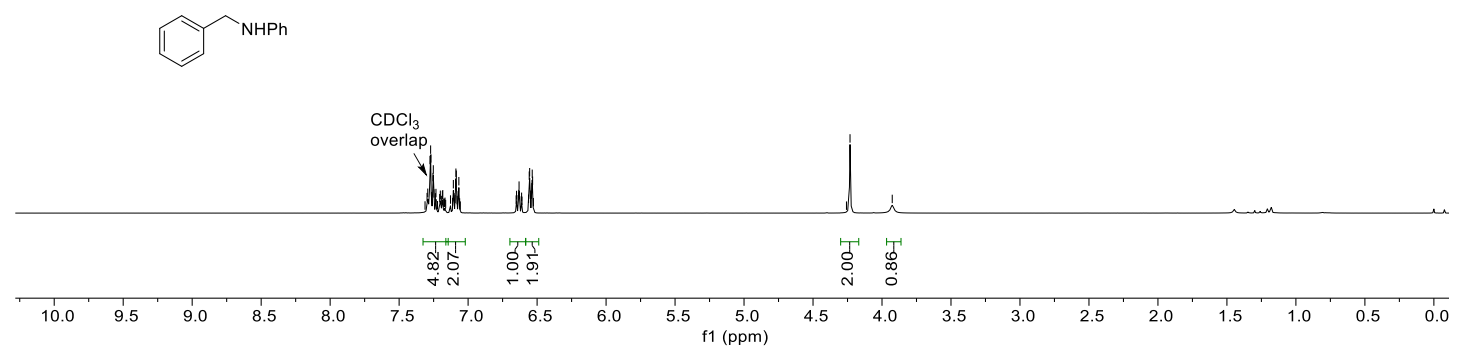

2bx; ${ }^{13} \mathrm{C}$ NMR; $\mathrm{CDCl}_{3} ; 101 \mathrm{MHz}$ 

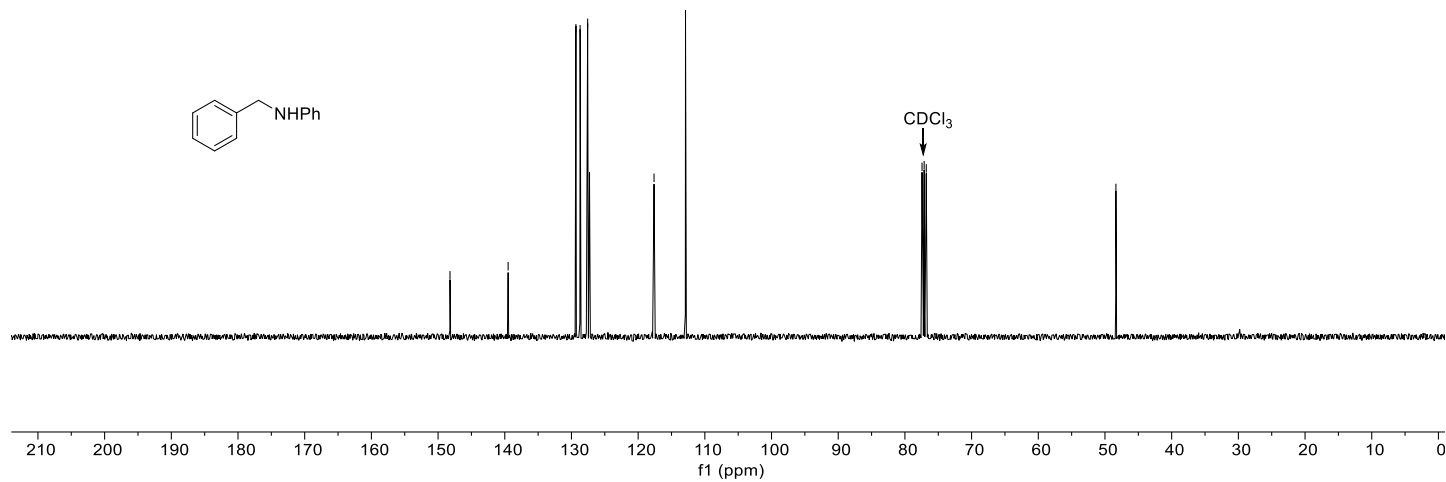

2by; ${ }^{1} \mathrm{H}$ NMR; $\mathrm{CDCl}_{3} ; 400 \mathrm{MHz}$

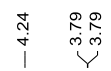

MeO

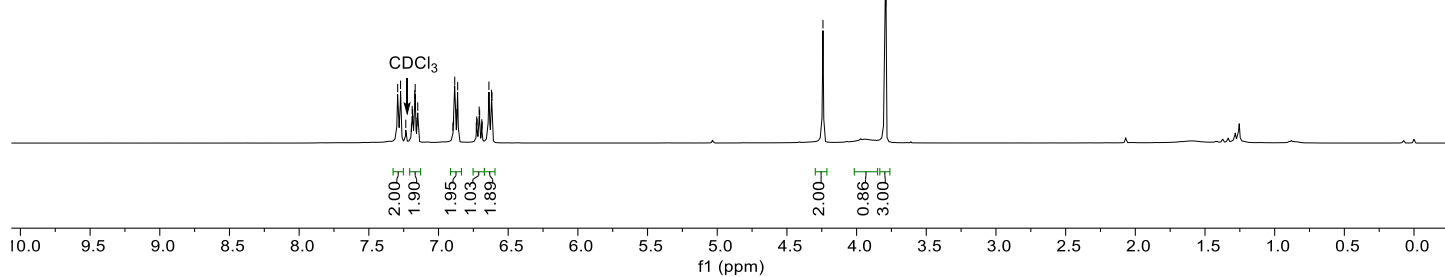

2by; ${ }^{13} \mathrm{C} \mathrm{NMR} ; \mathrm{CDCl}_{3} ; 101 \mathrm{MHz}$ 


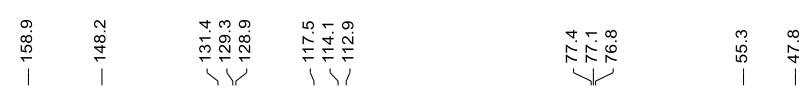

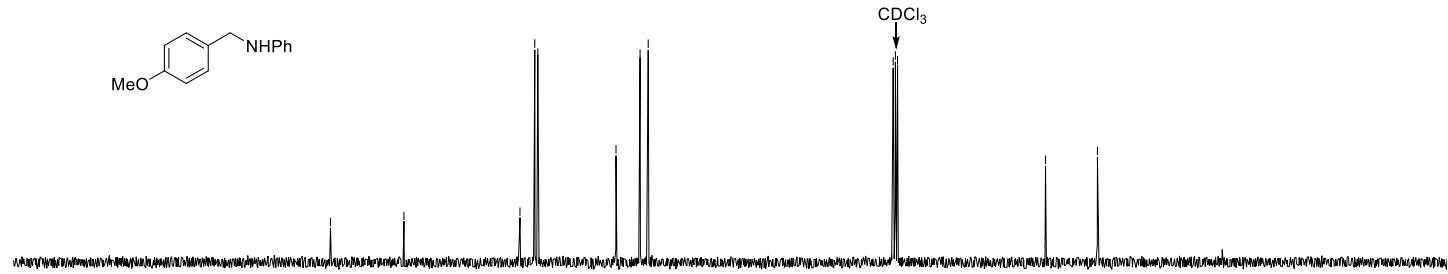

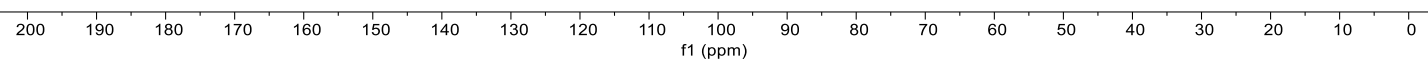

2bz; ${ }^{1} \mathrm{H}$ NMR; $\mathrm{CDCl}_{3} ; 400 \mathrm{MHz}$

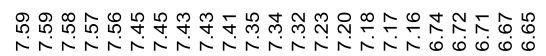

$\stackrel{m}{\stackrel{m}{+}} \stackrel{\substack{+i}}{i}$

8
0
1

NHPh

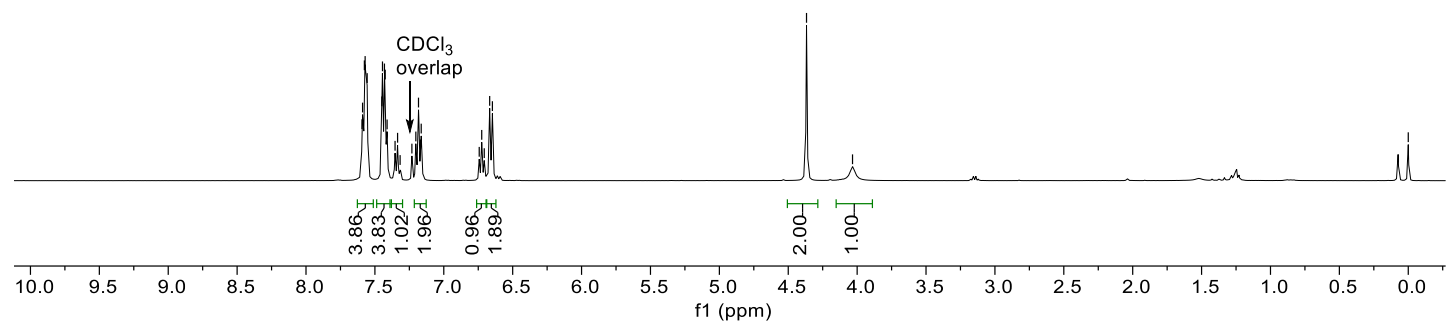

2bz; ${ }^{13} \mathrm{C}$ NMR; $\mathrm{CDCl}_{3} ; 101 \mathrm{MHz}$ 


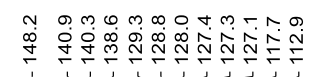

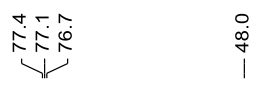

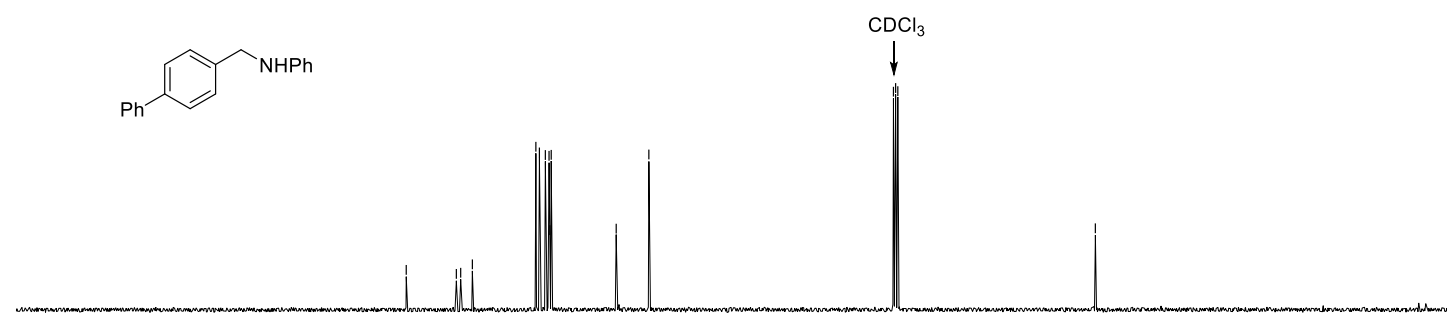

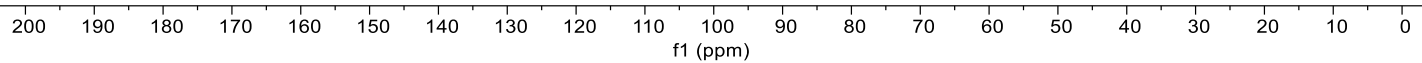

d-2aa; ${ }^{1} \mathrm{H} \mathrm{NMR} ; \mathrm{CDCl}_{3} ; 400 \mathrm{MHz}$

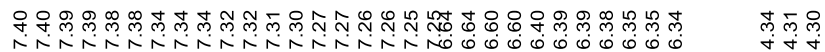

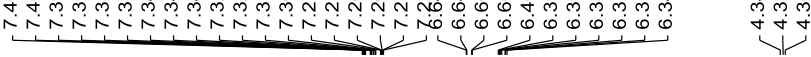

$\curvearrowright \mathrm{CHDOH}$

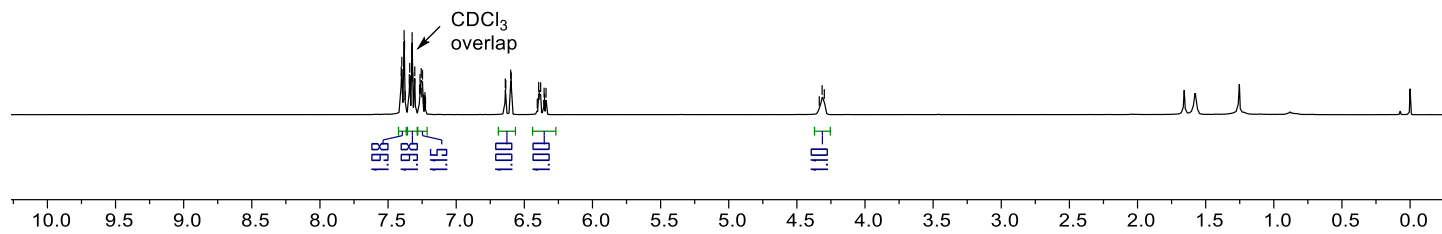

$d$-2aa; $;{ }^{13} \mathrm{C} \mathrm{NMR} ; \mathrm{CDCl}_{3} ; 101 \mathrm{MHz}$ 


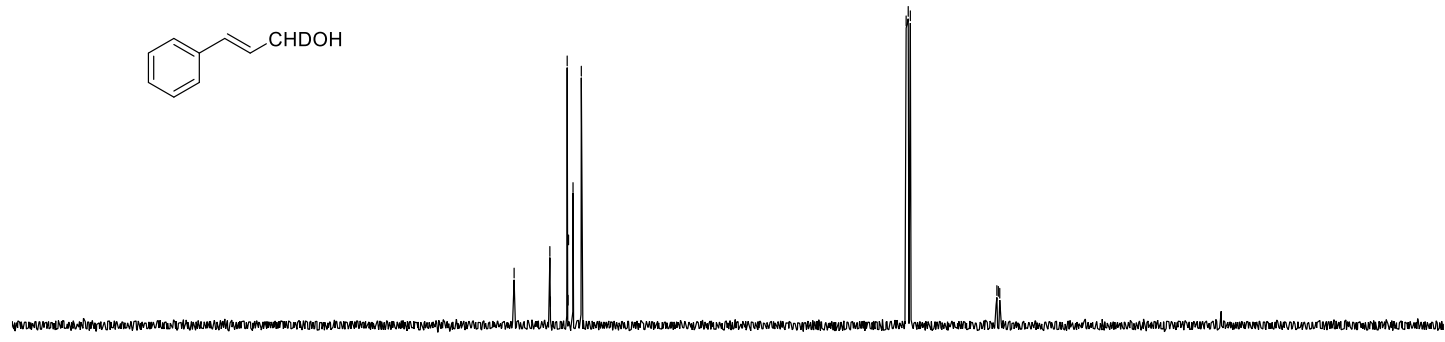

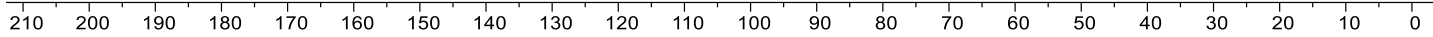

2ca; ${ }^{1} \mathrm{H} \mathrm{NMR} ; \mathrm{CDCl}_{3} ; 400 \mathrm{MHz}$ 


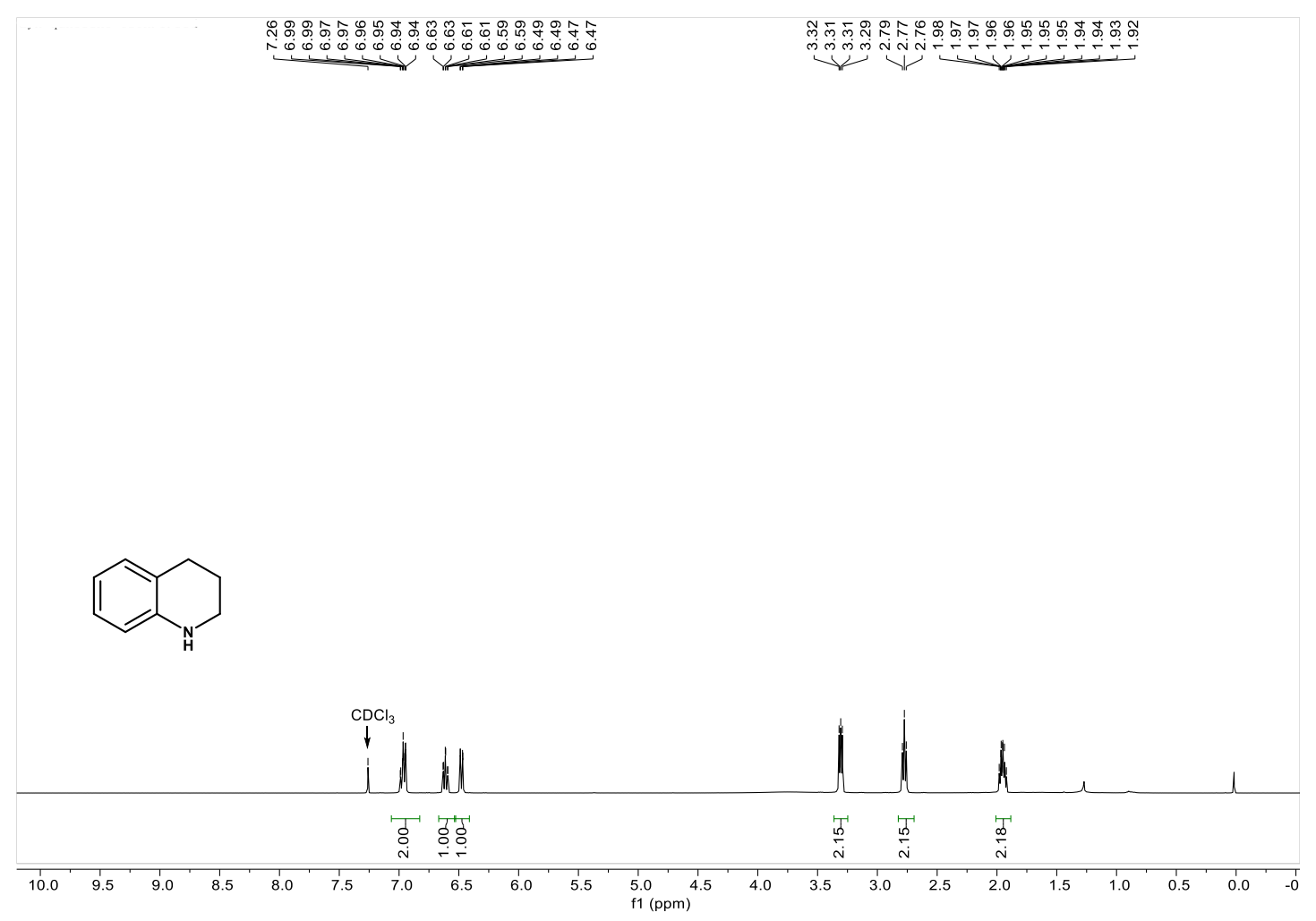

2ca; ${ }^{13} \mathrm{C} \mathrm{NMR} ; \mathrm{CDCl}_{3} ; 101 \mathrm{MHz}$

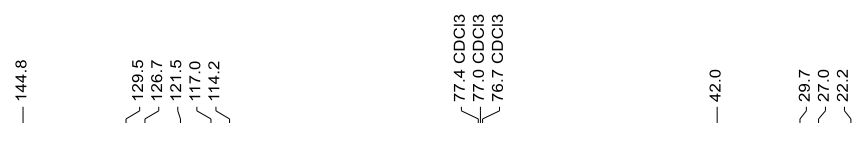

$\sqrt{n}$

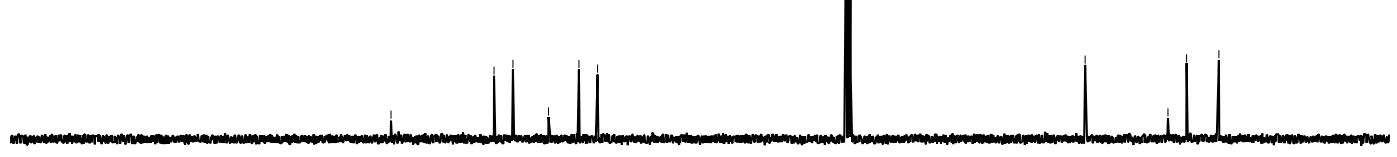

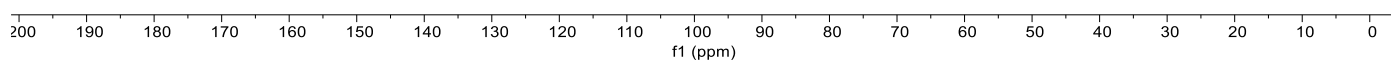

2cb; ${ }^{1} \mathrm{H} \mathrm{NMR} ; \mathrm{CDCl}_{3} ; 400 \mathrm{MHz}$ 


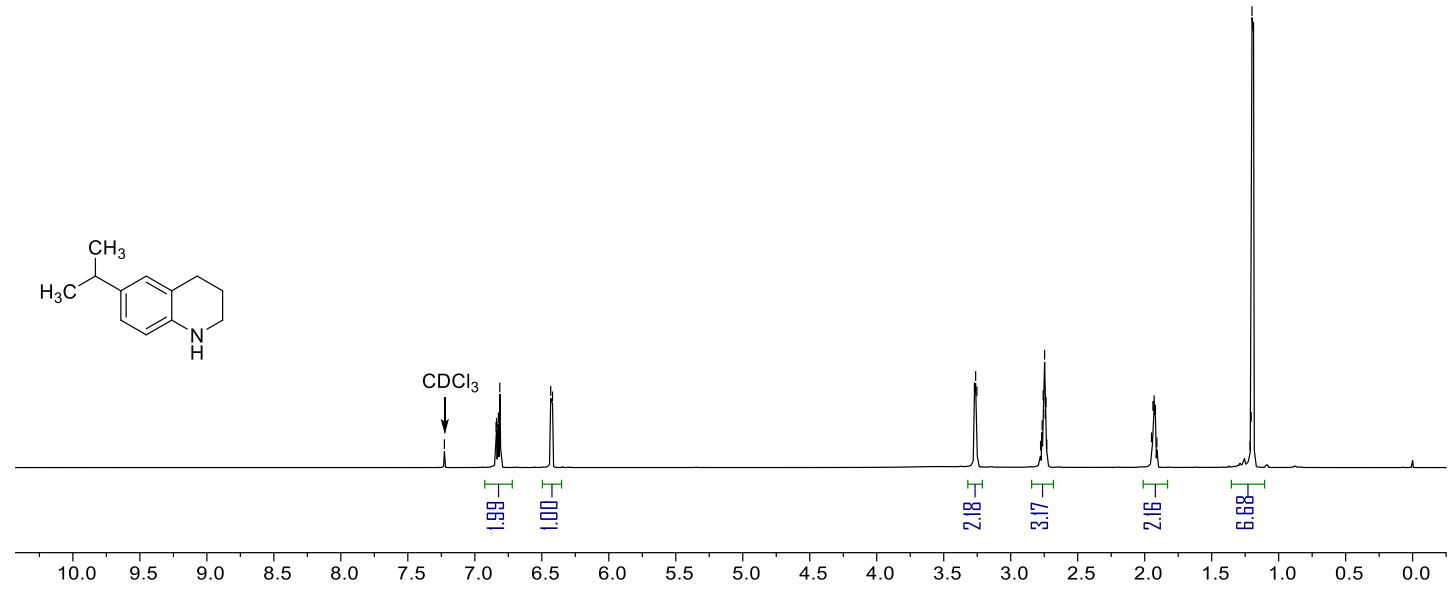

2cb; ${ }^{13} \mathrm{C}$ NMR; $\mathrm{CDCl}_{3} ; 101 \mathrm{MHz}$

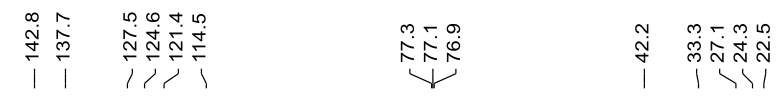

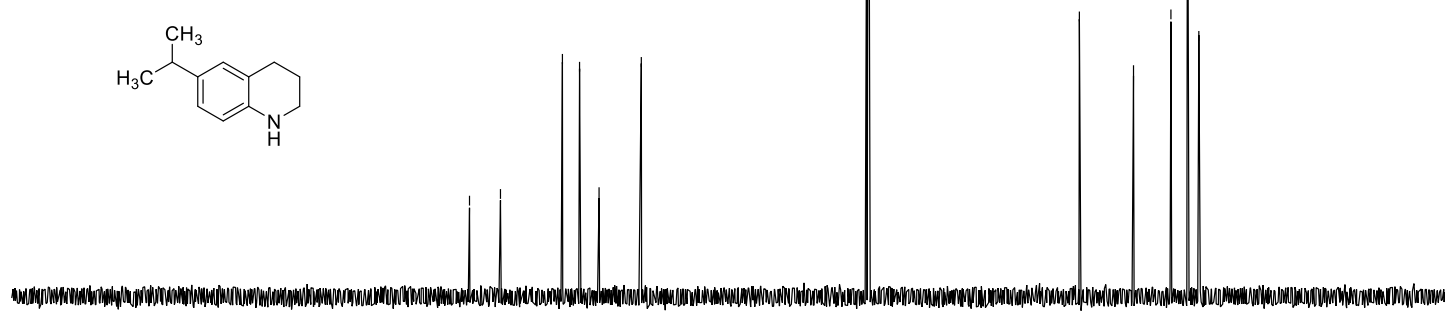

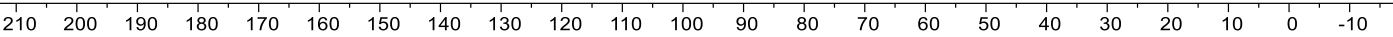

2cc; ${ }^{1} \mathrm{H} \mathrm{NMR} ; \mathrm{CDCl}_{3} ; 400 \mathrm{MHz}$ 


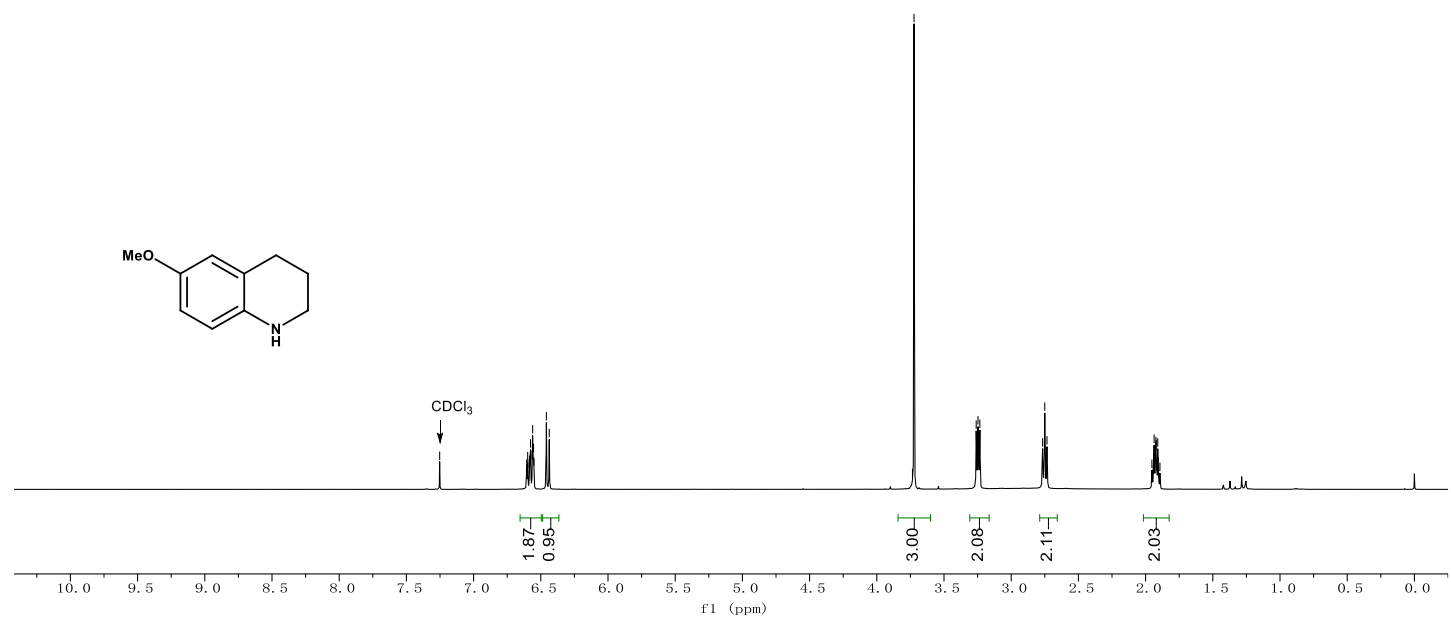

2cc; ${ }^{13} \mathrm{C}$ NMR; $\mathrm{CDCl}_{3} ; 101 \mathrm{MHz}$

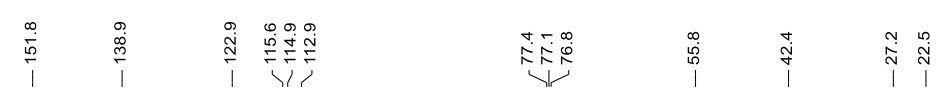

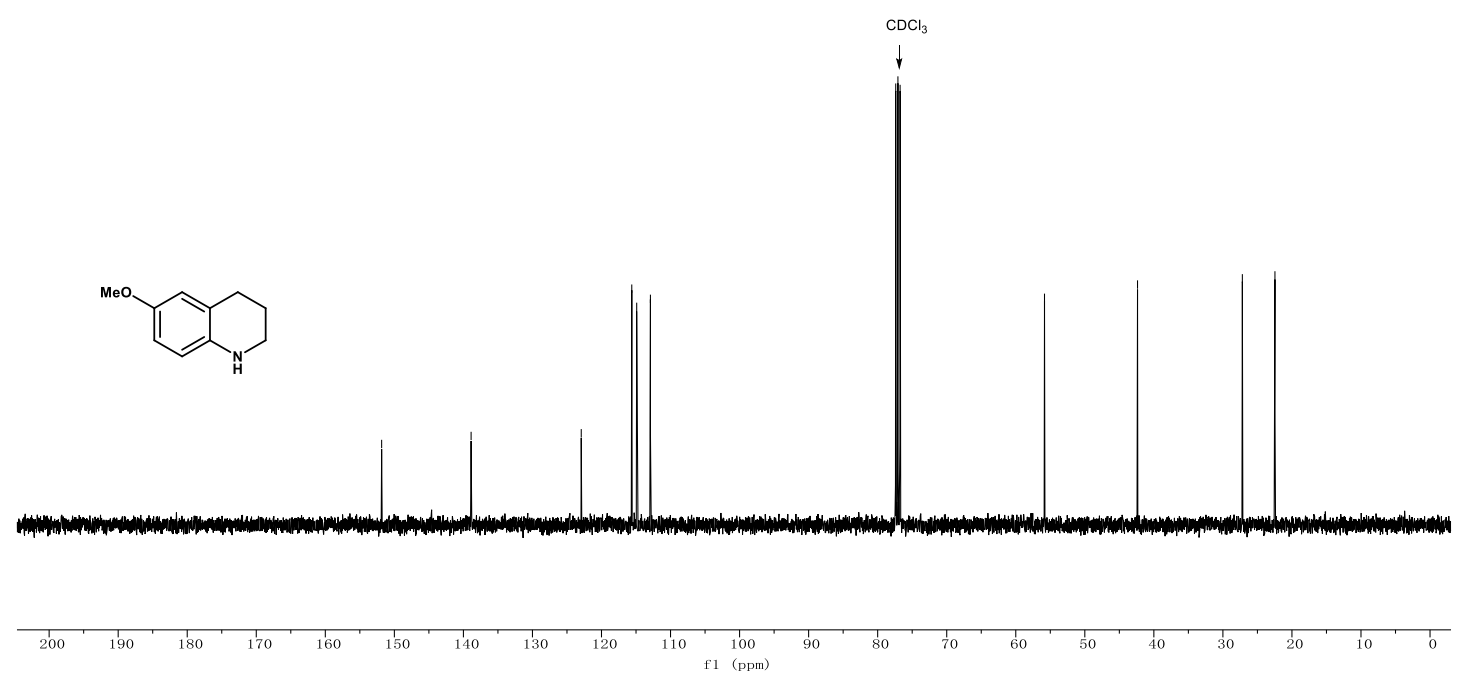

2cd; ${ }^{1} \mathrm{H}$ NMR; $\mathrm{CDCl}_{3} ; 400 \mathrm{MHz}$ 

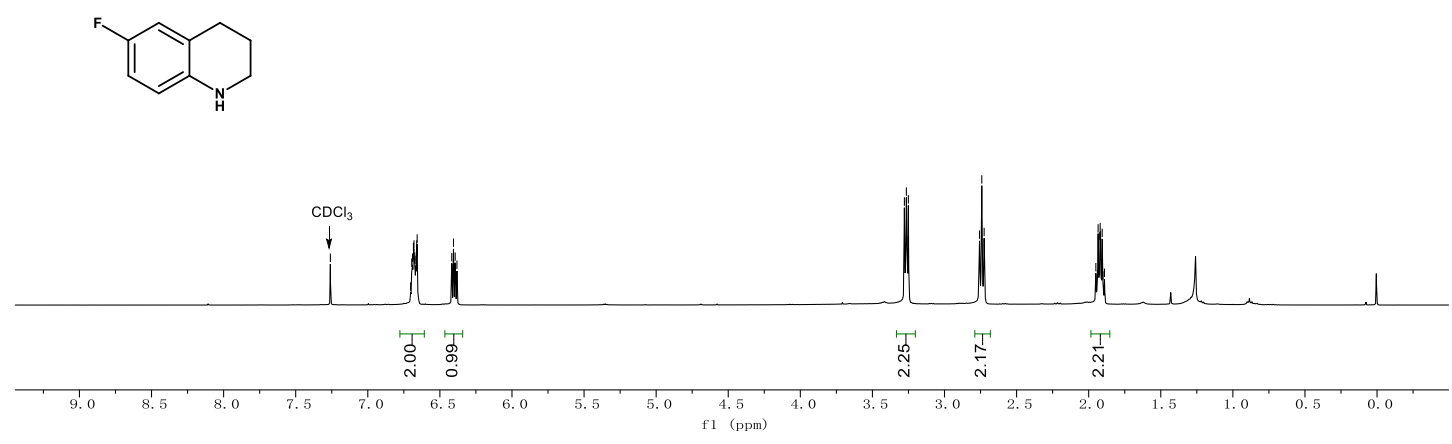

2cd; ${ }^{13} \mathrm{C}$ NMR; $\mathrm{CDCl}_{3} ; 101 \mathrm{MHz}$

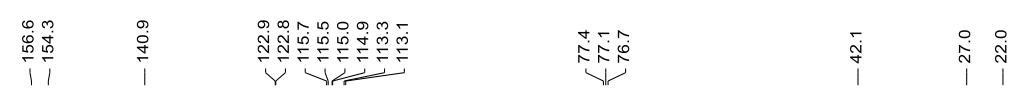<smiles>Ic1ccc2c(c1)CCC[Te]2</smiles>

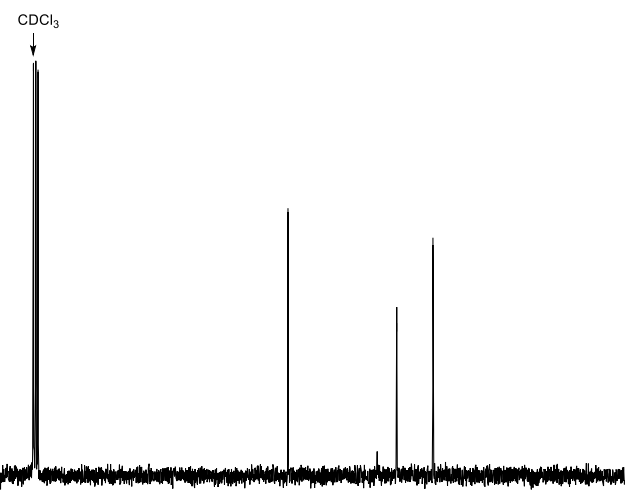

2cd; ${ }^{19} \mathrm{~F}$ NMR; $\mathrm{CDCl}_{3} ; 376 \mathrm{MHz}$ 

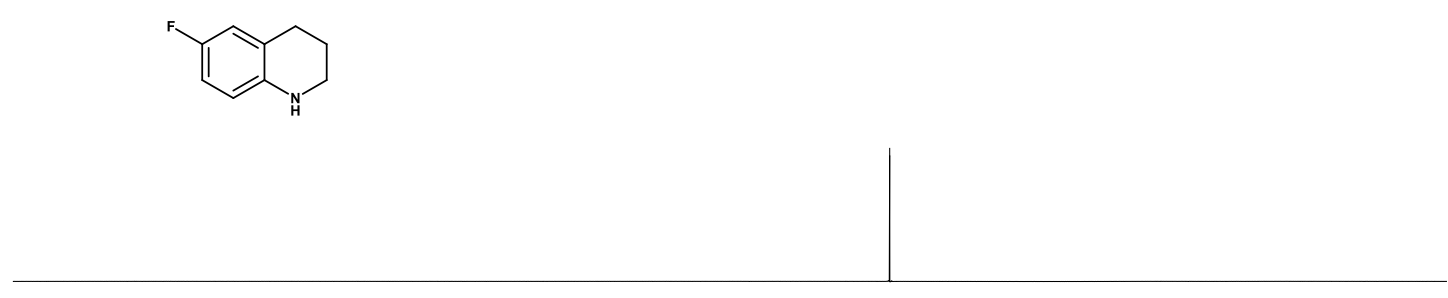

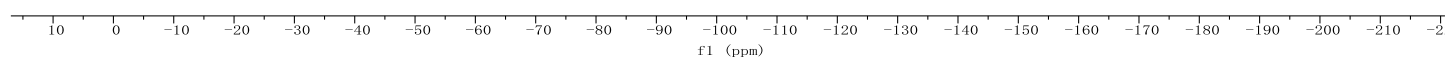

2ce; ${ }^{1} \mathrm{H} \mathrm{NMR} ; \mathrm{CDCl}_{3} ; 400 \mathrm{MHz}$

祝:

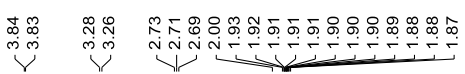

(1)

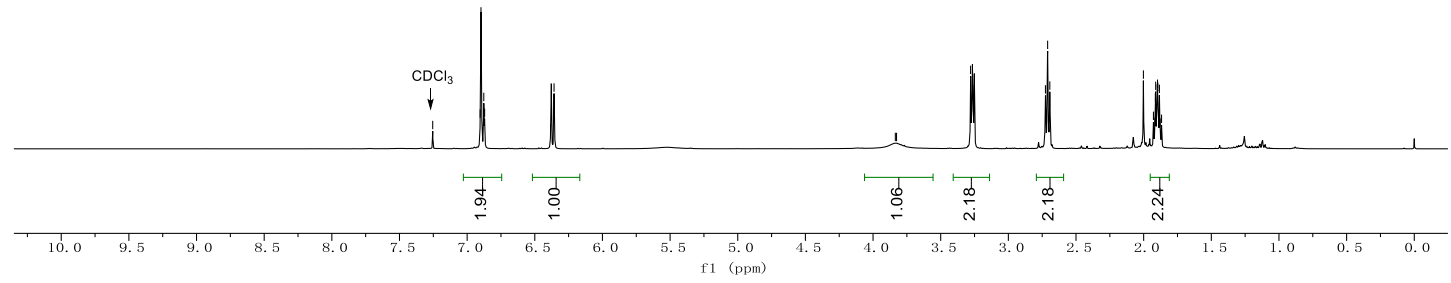

2ce; ${ }^{13} \mathrm{C} \mathrm{NMR} ; \mathrm{CDCl}_{3} ; 101 \mathrm{MHz}$ 
1
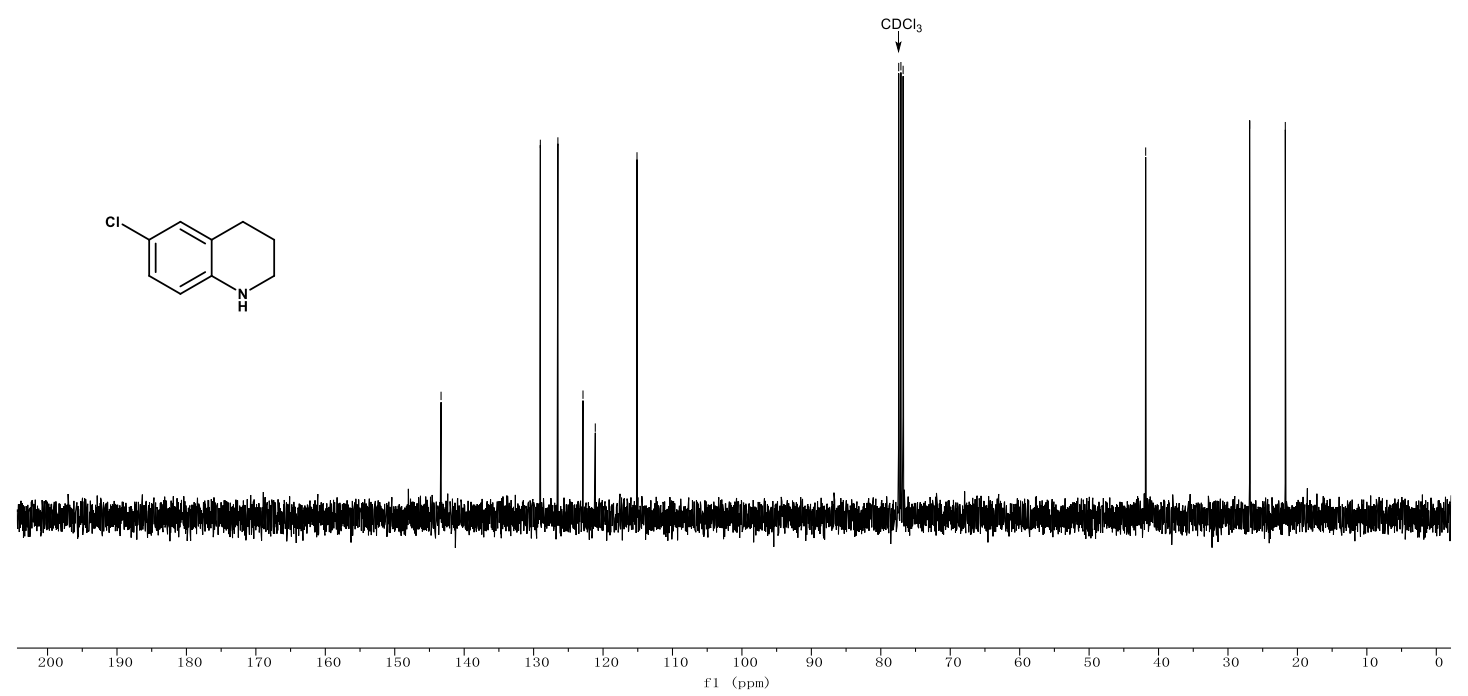

2ef; ${ }^{1} \mathrm{H}$ NMR; $\mathrm{CDCl}_{3} ; 600 \mathrm{MHz}$

\begin{tabular}{|c|c|c|}
\hline 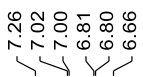 & 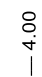 & 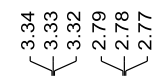 \\
\hline
\end{tabular}

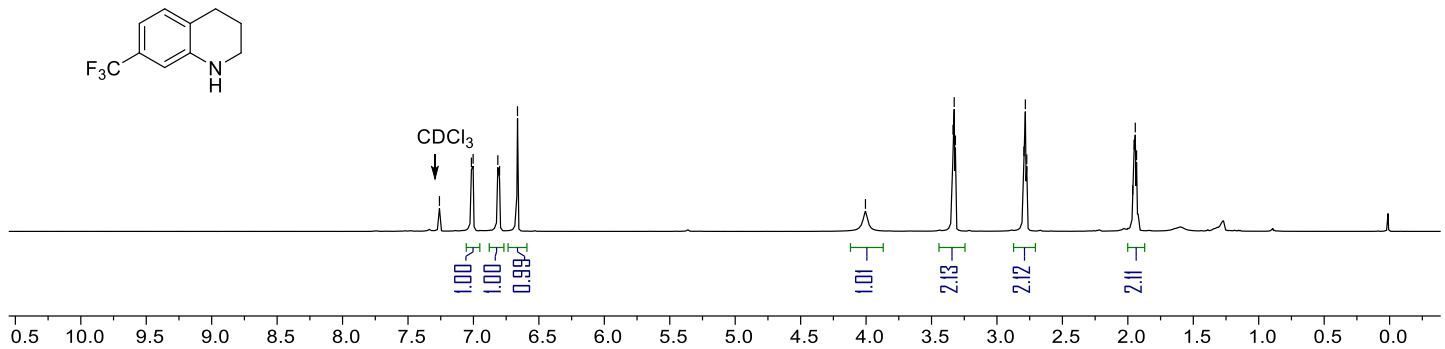

2cf; ${ }^{19} \mathrm{~F} \mathrm{NMR} ; \mathrm{CDCl}_{3} ; 565 \mathrm{MHz}$ 

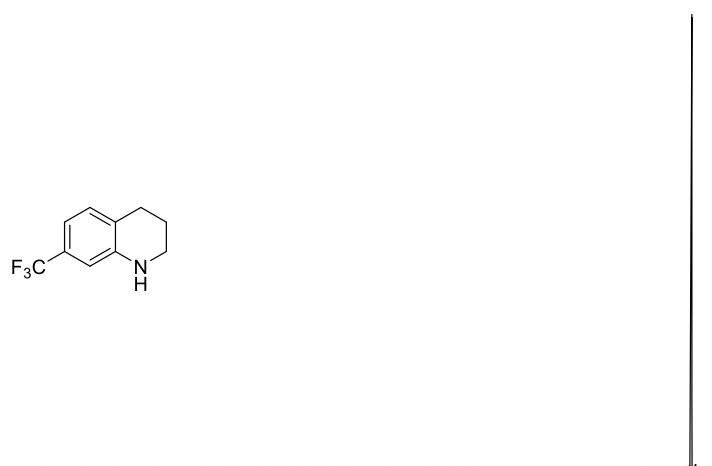

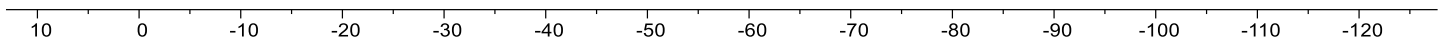

2ef; ${ }^{13} \mathrm{C} \mathrm{NMR} ; \mathrm{CDCl}_{3} ; 151 \mathrm{MHz}$

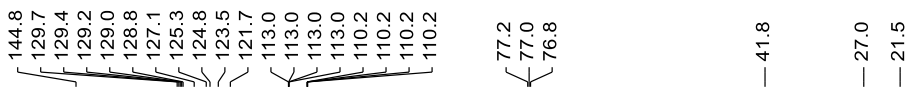

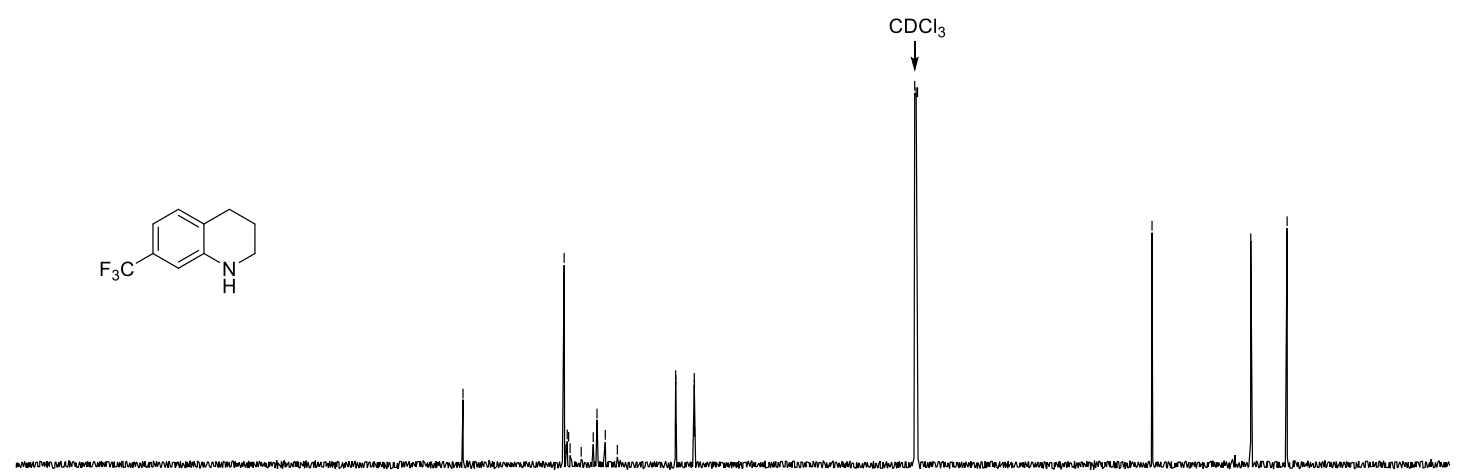

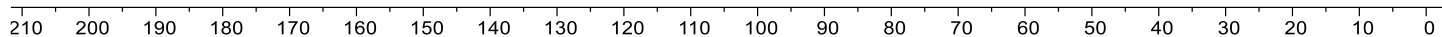

2cg; ${ }^{1} \mathrm{H} \mathrm{NMR} ; \mathrm{CDCl}_{3} ; 400 \mathrm{MHz}$ 


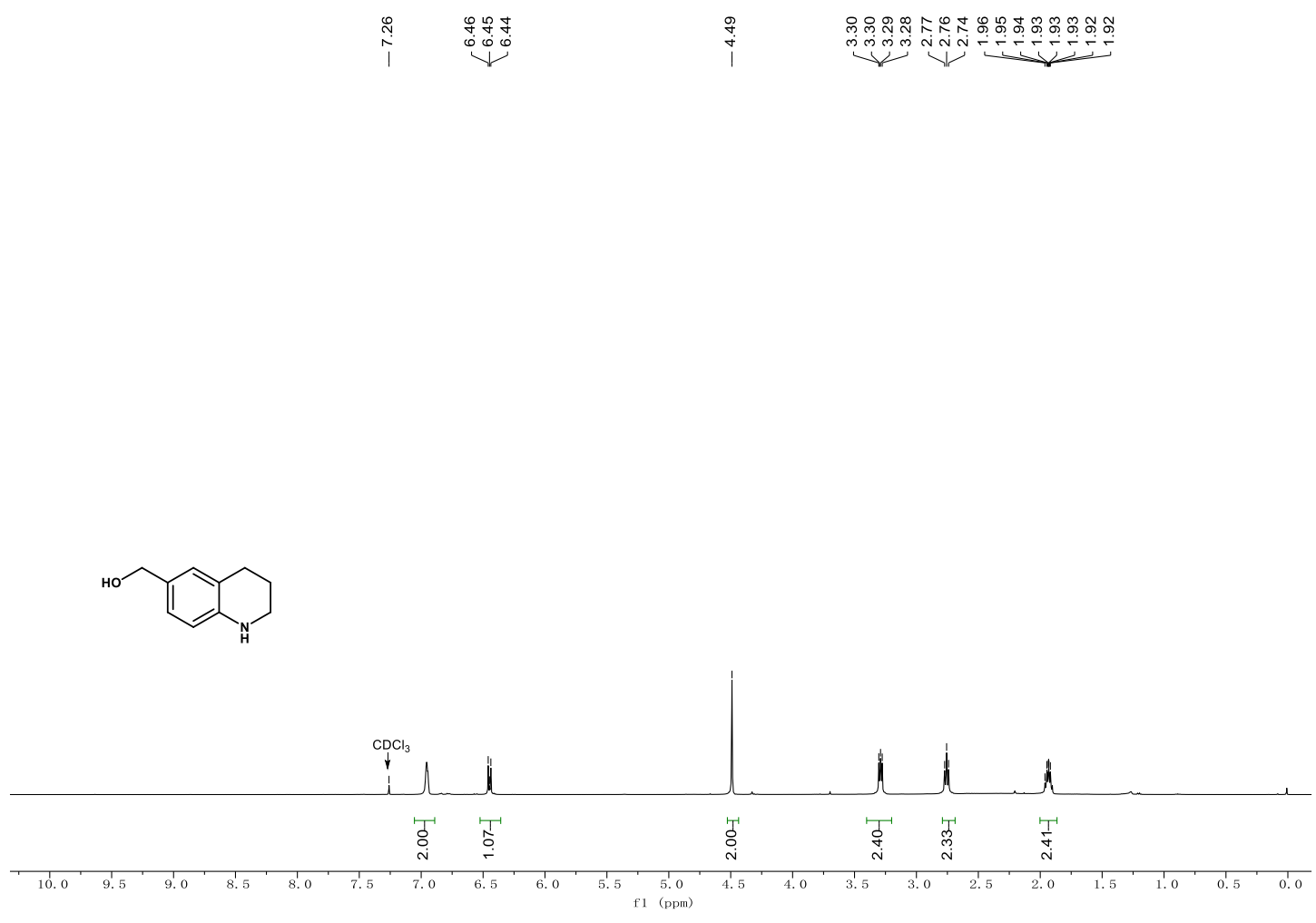

2cg; ${ }^{13} \mathrm{C}$ NMR; $\mathrm{CDCl}_{3} ; 101 \mathrm{MHz}$

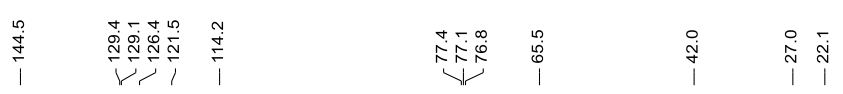

Ho

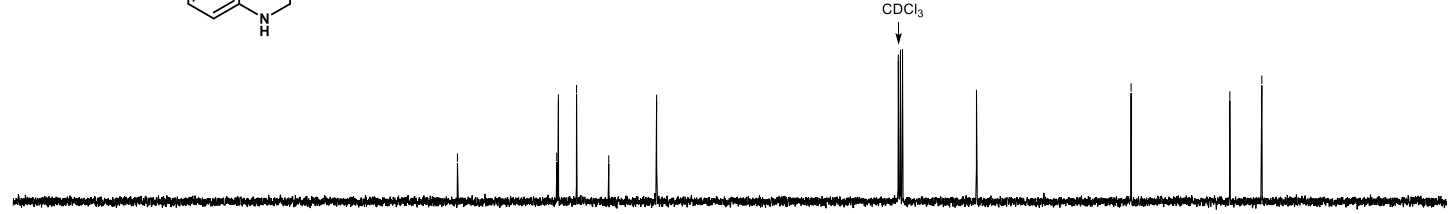

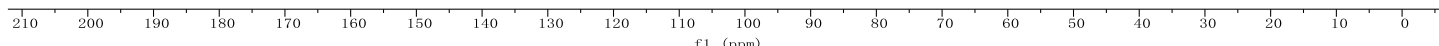

2ch; ${ }^{1} \mathrm{H}$ NMR; $\mathrm{CDCl}_{3} ; 400 \mathrm{MHz}$ 


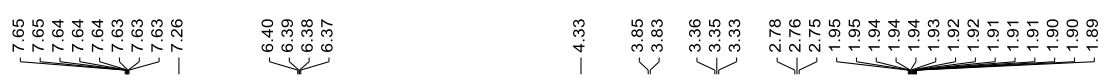

Moo 2 C

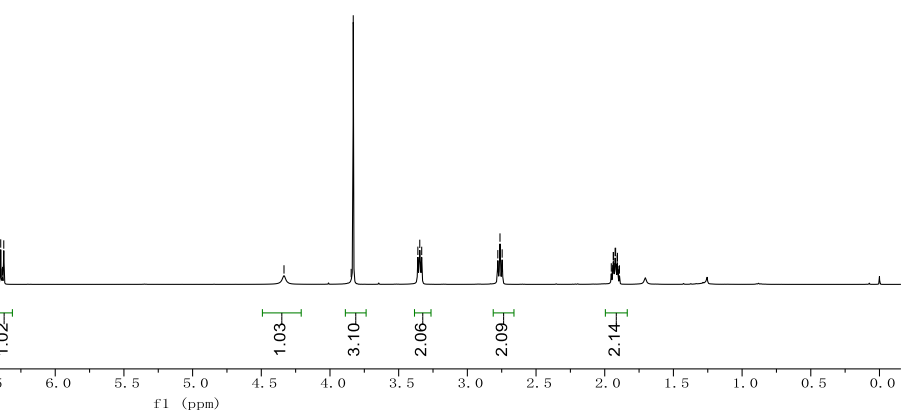

2ch; ${ }^{13} \mathrm{C}$ NMR; $\mathrm{CDCl}_{3} ; 101 \mathrm{MHz}$

每

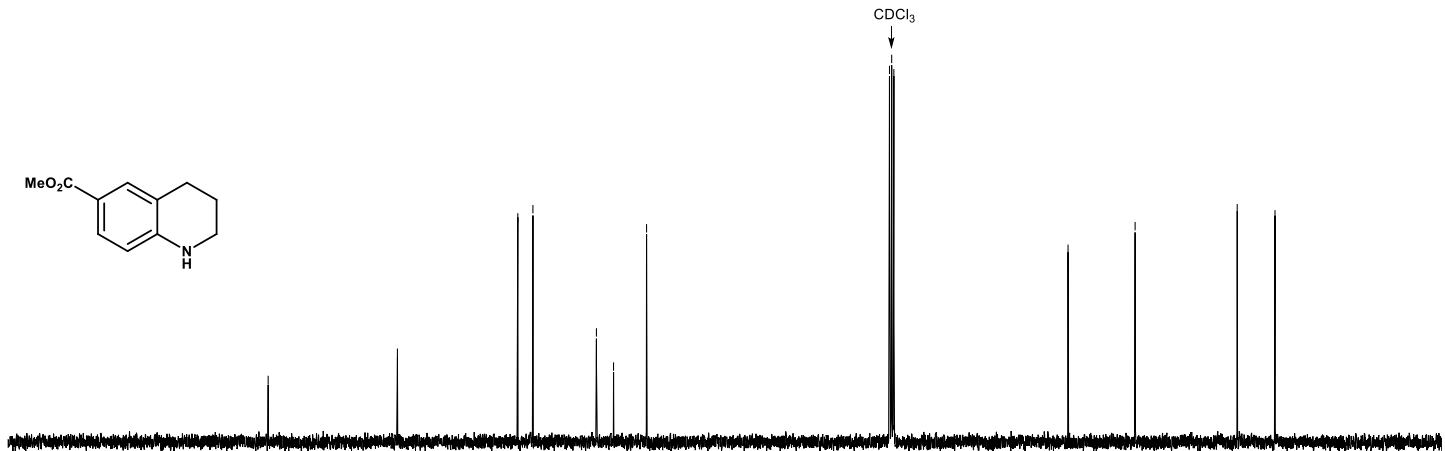

2ci; ${ }^{1} \mathrm{H}$ NMR; $\mathrm{CDCl}_{3} ; 400 \mathrm{MHz}$ 

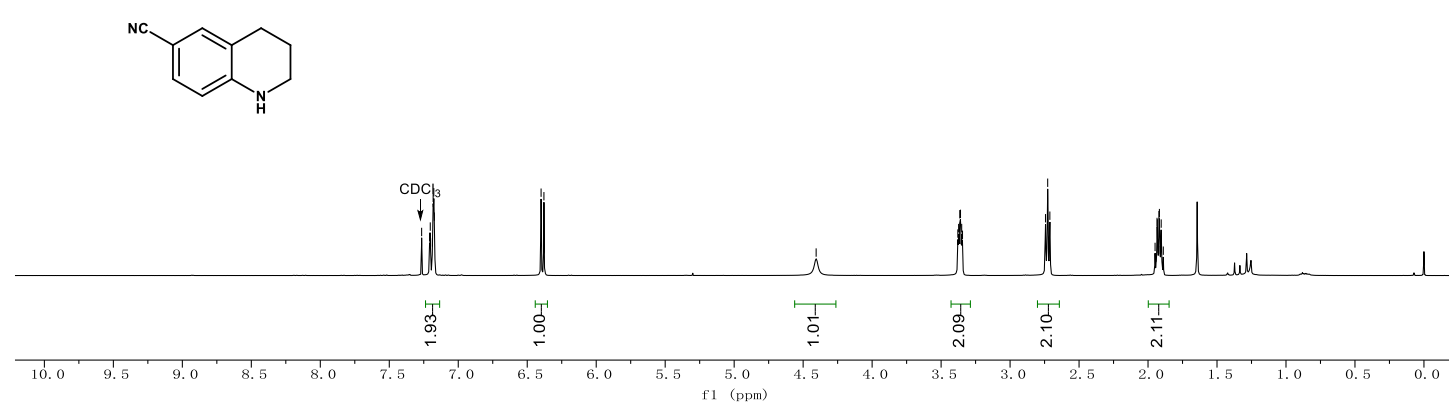

2ci; ${ }^{13} \mathrm{C} \mathrm{NMR} ; \mathrm{CDCl}_{3} ; 101 \mathrm{MHz}$

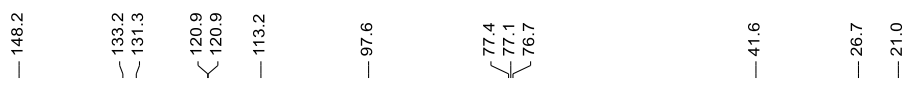
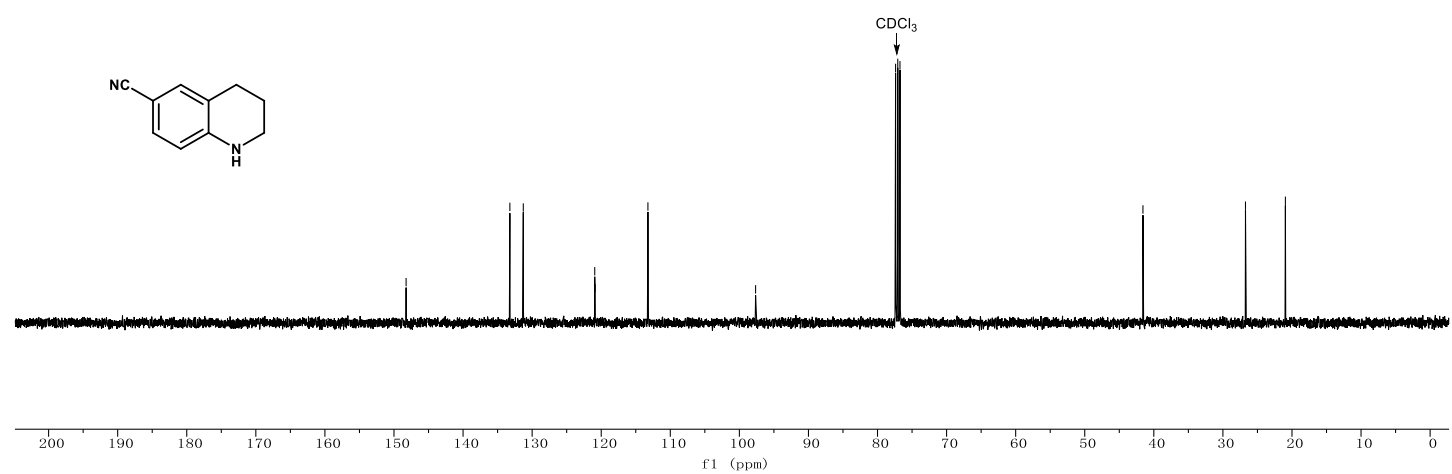

2cj; ${ }^{1} \mathrm{H} \mathrm{NMR} ; \mathrm{CDCl}_{3} ; 600 \mathrm{MHz}$ 

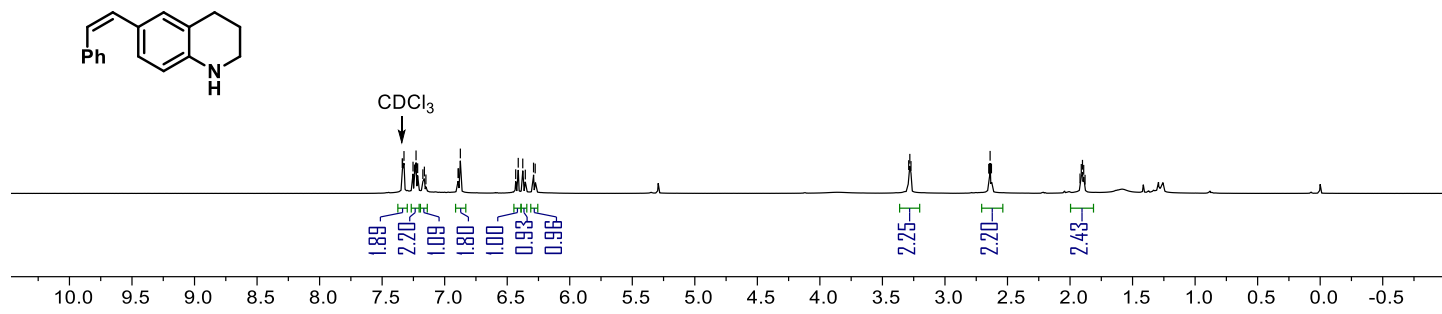

2cj; ${ }^{13} \mathrm{C} \mathrm{NMR} ; \mathrm{CDCl}_{3} ; 151 \mathrm{MHz}$

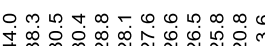

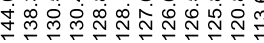

年

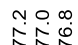

$\sqrt[n]{4}$

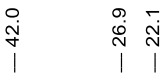

Ph

$\mathrm{CDCl}_{3}$

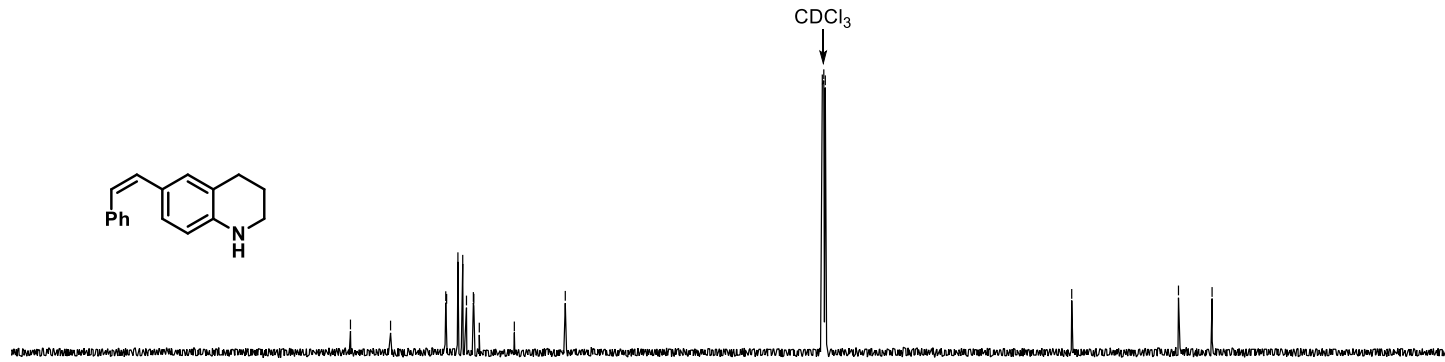

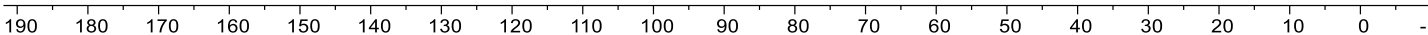


2ck; ${ }^{1} \mathrm{H} \mathrm{NMR} ; \mathrm{CDCl}_{3} ; 600 \mathrm{MHz}$

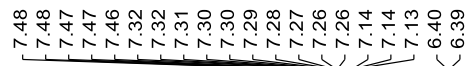

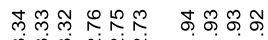

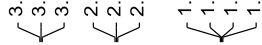

(N)

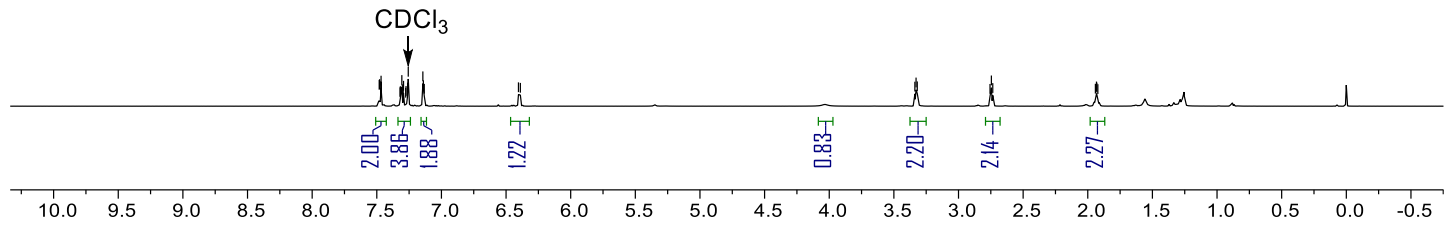

2ck; ${ }^{13} \mathrm{C} \mathrm{NMR} ; \mathrm{CDCl}_{3} ; 151 \mathrm{MHz}$

I

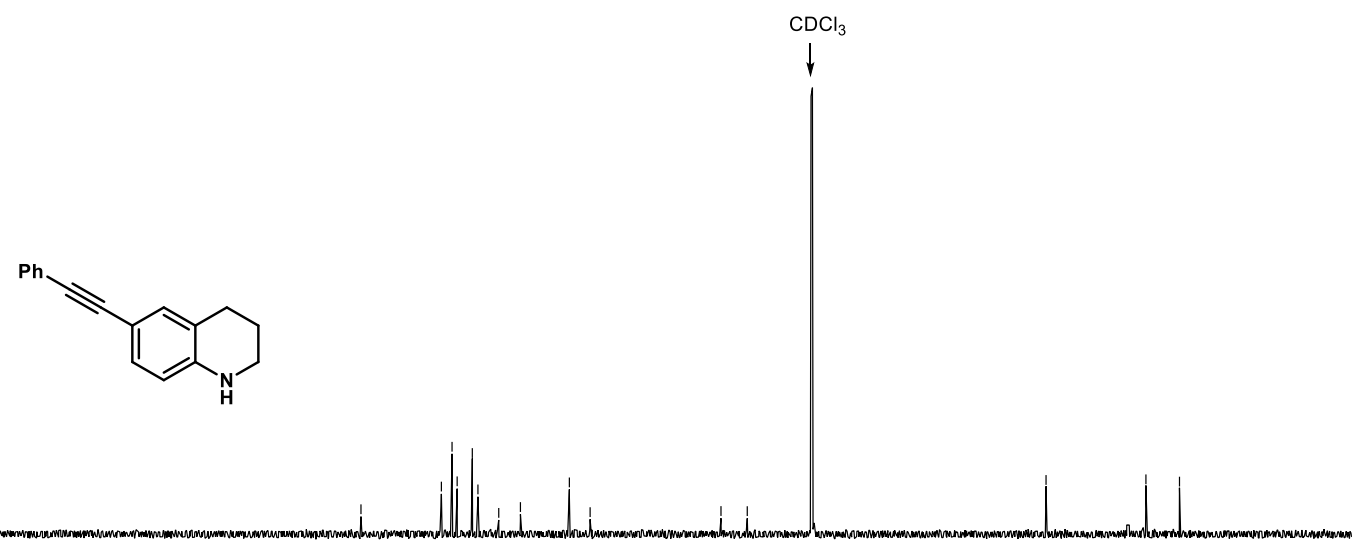

\begin{tabular}{lllllllllllllllllllllllll}
\hline 210 & 200 & 190 & 180 & 170 & 160 & 150 & 140 & 130 & 120 & 110 & 100 & 90 & 80 & 70 & 60 & 50 & 40 & 30 & 20 & 10 & 0
\end{tabular}

2cl; ${ }^{1} \mathrm{H}$ NMR; $\mathrm{CDCl}_{3} ; 400 \mathrm{MHz}$ 


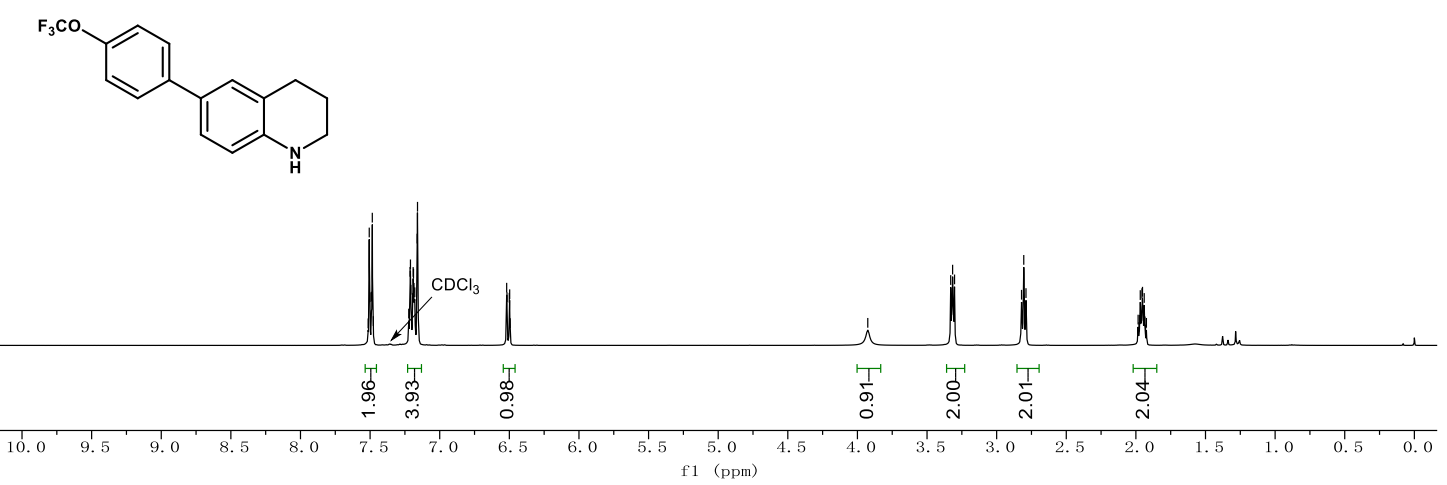

2cl; ${ }^{13} \mathrm{C} \mathrm{NMR} ; \mathrm{CDCl}_{3} ; 101 \mathrm{MHz}$

il
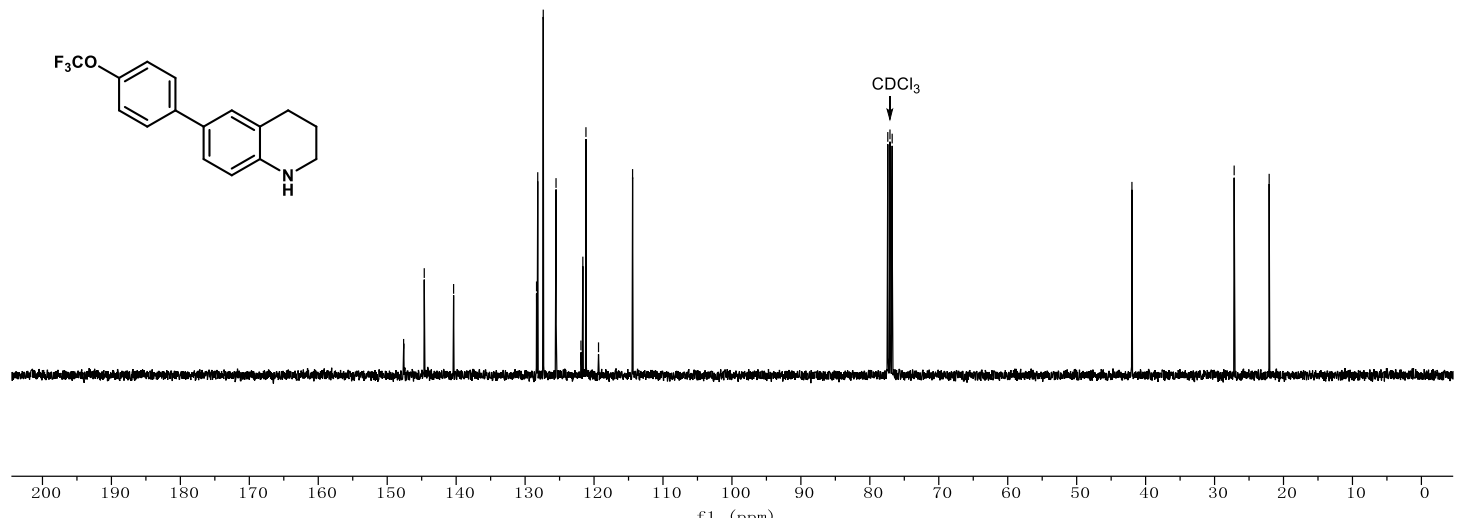

2cl; ${ }^{19} \mathrm{~F}$ NMR; $\mathrm{CDCl}_{3} ; 376 \mathrm{MHz}$ 

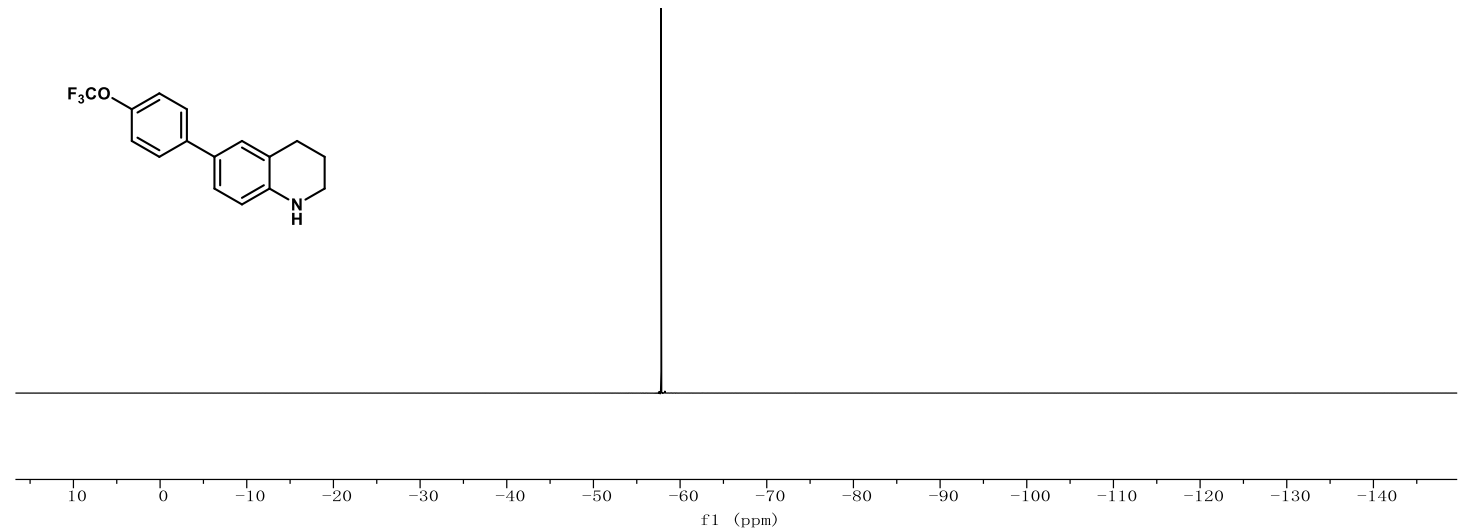

2cm; ${ }^{1} \mathrm{H}$ NMR; $\mathrm{CDCl}_{3} ; 600 \mathrm{MHz}$
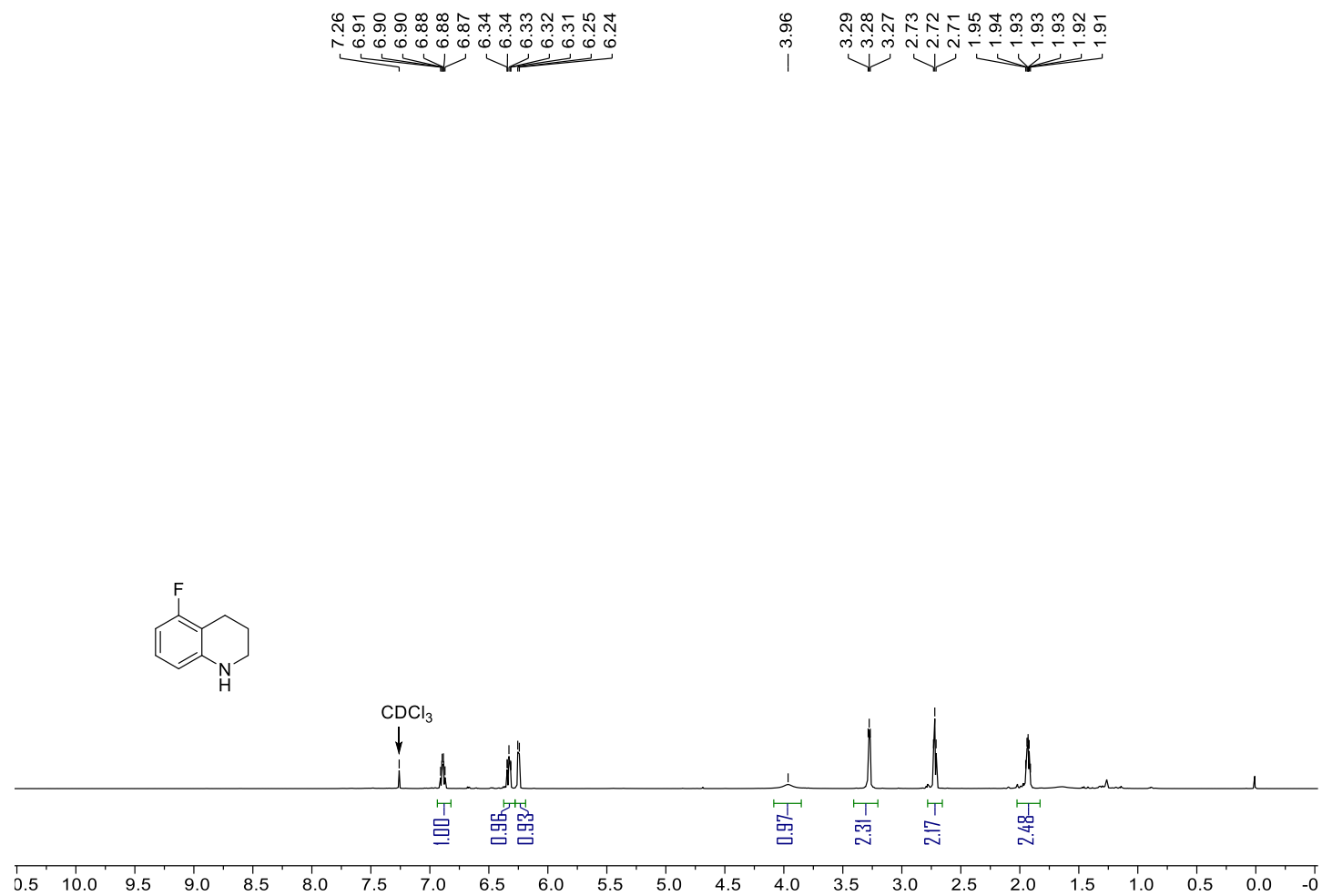

2em; ${ }^{19} \mathrm{~F} \mathrm{NMR} ; \mathrm{CDCl}_{3} ; 565 \mathrm{MHz}$ 


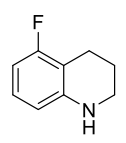

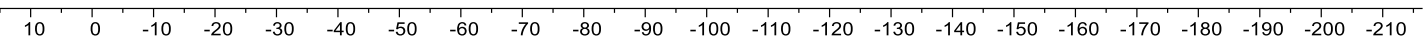

$\mathbf{2 c m} ;{ }^{13} \mathrm{C} \mathrm{NMR} ; \mathrm{CDCl}_{3} ; 151 \mathrm{MHz}$
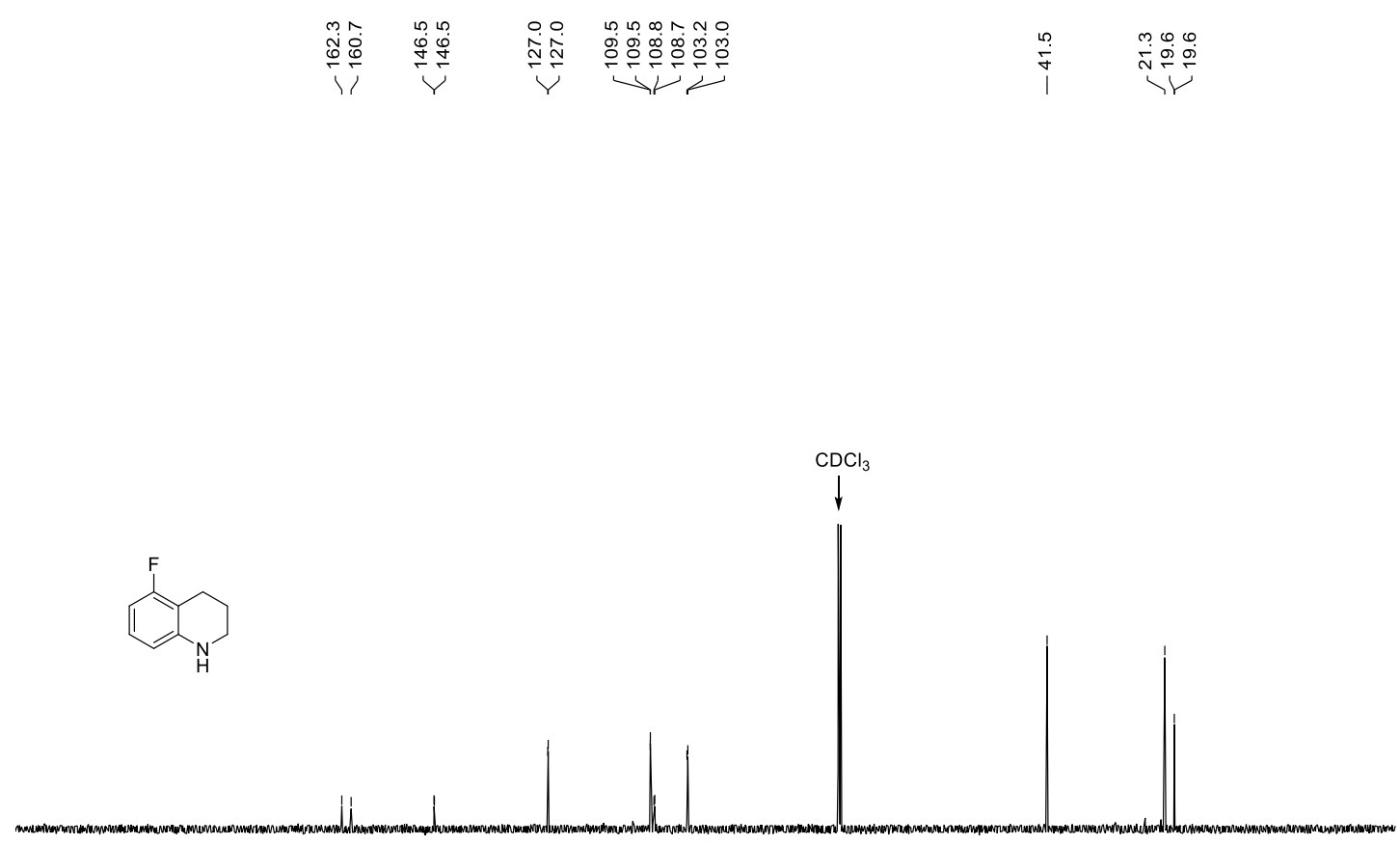

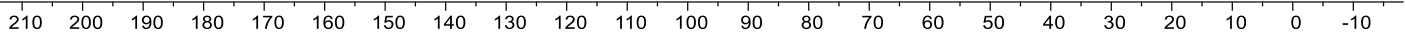

2cn; ${ }^{1} \mathrm{H}$ NMR; $\mathrm{CDCl}_{3} ; 400 \mathrm{MHz}$ 
i<smiles>[Tl]c1ccc2c(c1)CCC2</smiles>

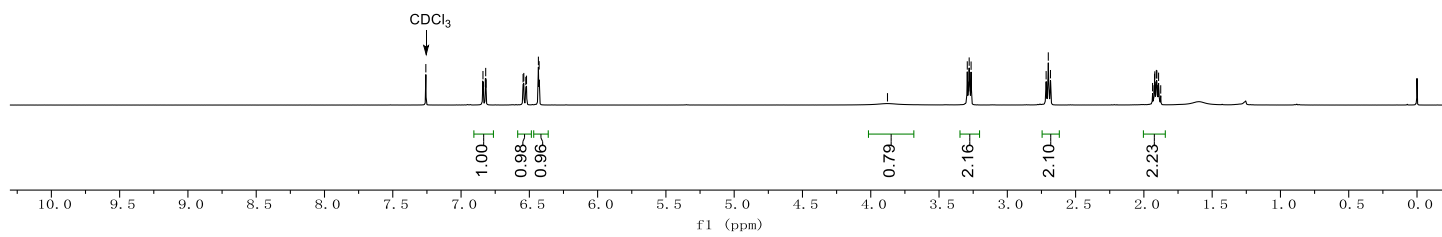

2cn; ${ }^{13} \mathrm{C}$ NMR; $\mathrm{CDCl}_{3} ; 101 \mathrm{MHz}$

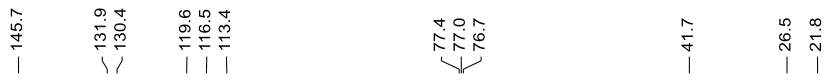<smiles>Clc1ccc2c(c1)[Te]CC2</smiles>

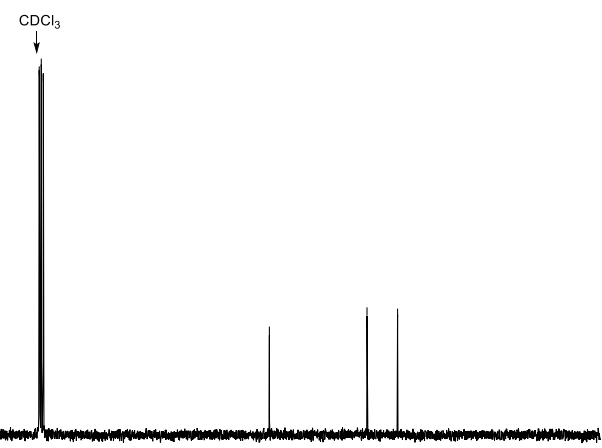

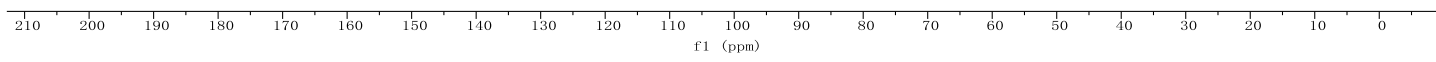


2co; ${ }^{1} \mathrm{H} \mathrm{NMR} ; \mathrm{CDCl}_{3} ; 600 \mathrm{MHz}$

N
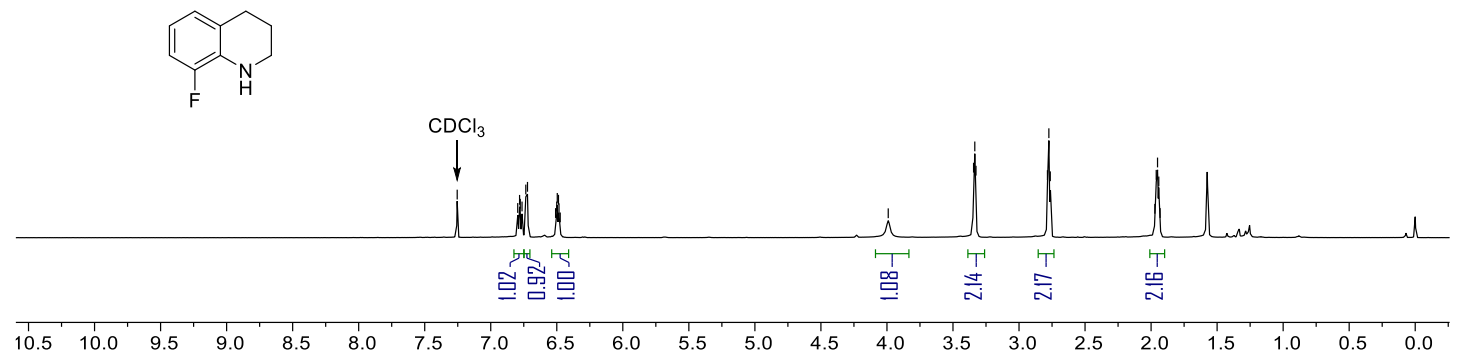

2co; ${ }^{19} \mathrm{~F} \mathrm{NMR} ; \mathrm{CDCl}_{3} ; 565 \mathrm{MHz}$

ì

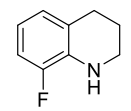

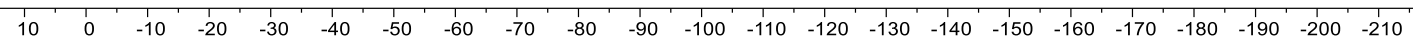


2co; ${ }^{13} \mathrm{C}$ NMR; $\mathrm{CDCl}_{3} ; 151 \mathrm{MHz}$
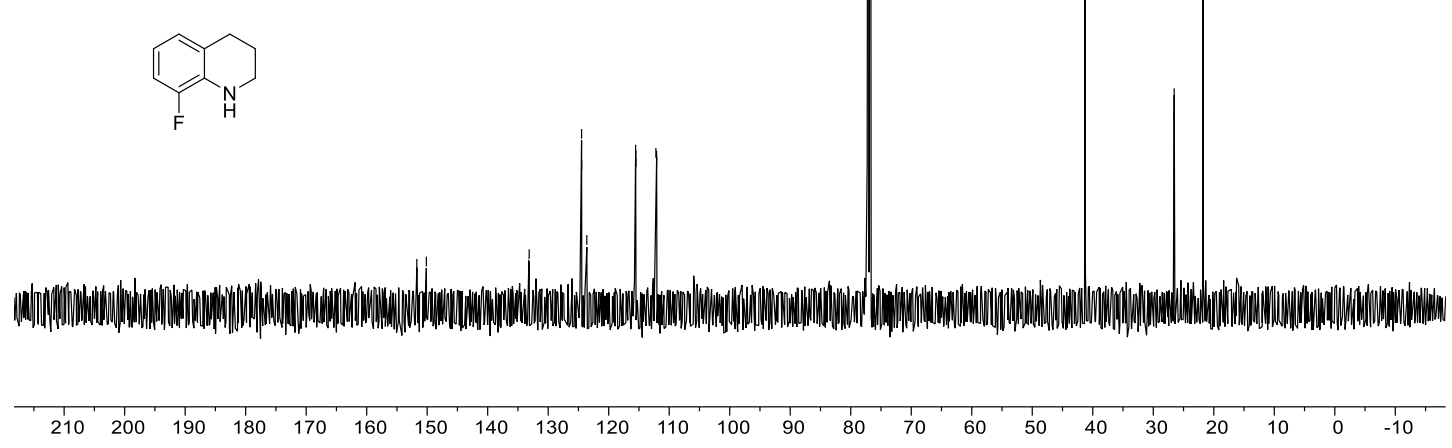

2cp; ${ }^{1} \mathrm{H}$ NMR; $\mathrm{CDCl}_{3} ; 400 \mathrm{MHz}$

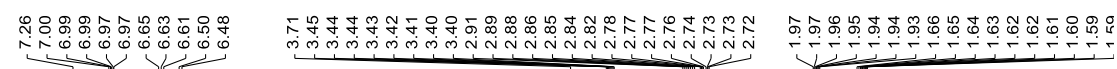
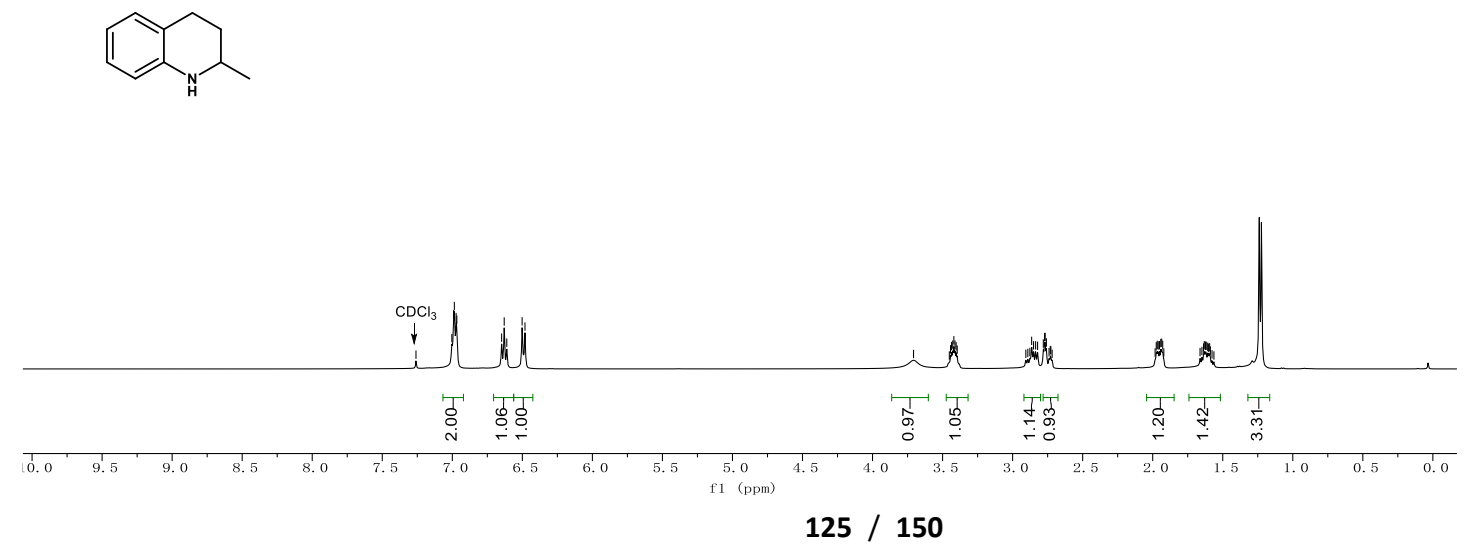
2cp; ${ }^{13} \mathrm{C} \mathrm{NMR} ; \mathrm{CDCl}_{3} ; 101 \mathrm{MHz}$

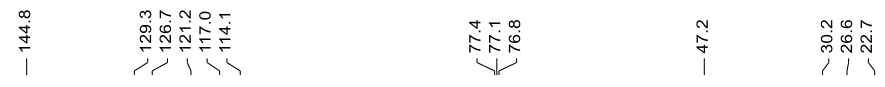<smiles>CC1CCc2ccccc2N1</smiles>
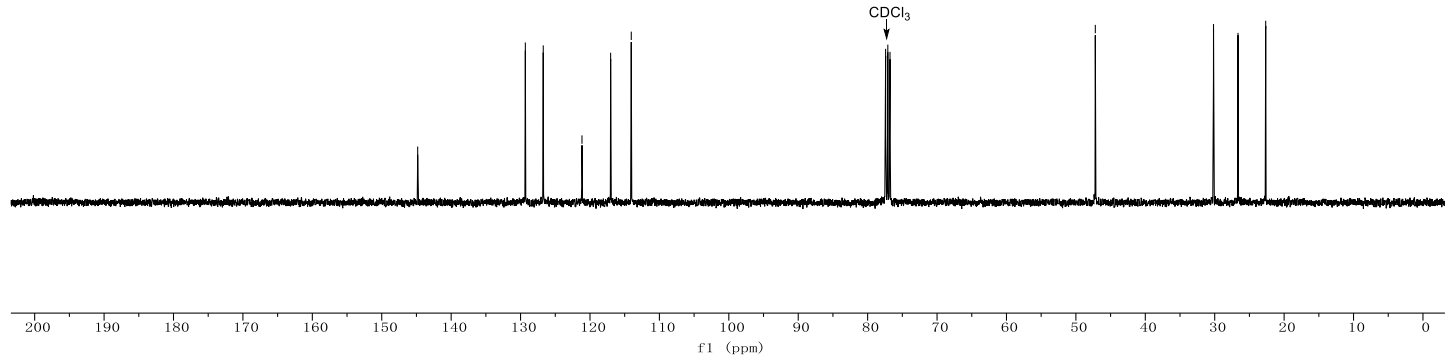

2cq; ${ }^{1} \mathrm{H} \mathrm{NMR} ; \mathrm{CDCl}_{3} ; 400 \mathrm{MHz}$

赵

$1 Y Y$ U
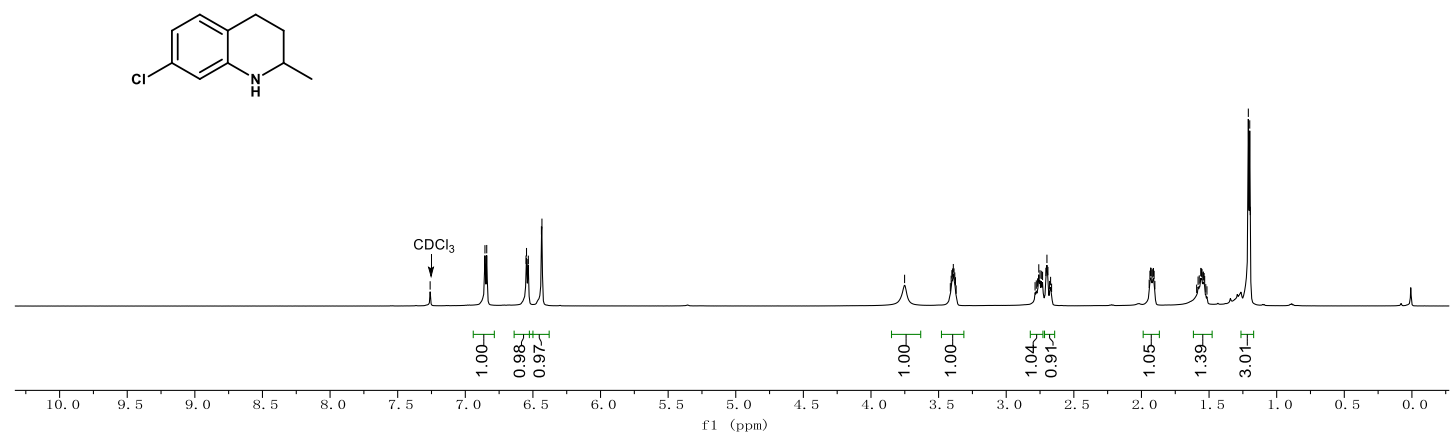
2cq; ${ }^{13} \mathrm{C} \mathrm{NMR} ; \mathrm{CDCl}_{3} ; 101 \mathrm{MHz}$

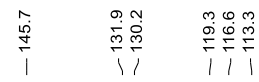

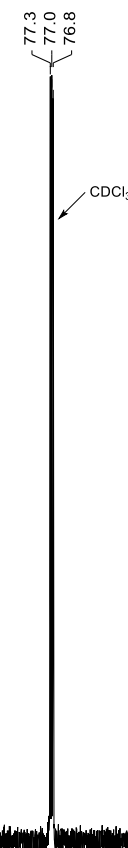

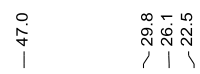

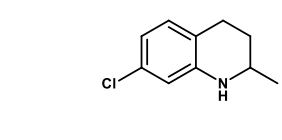

-

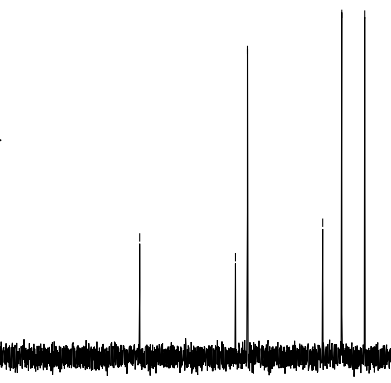

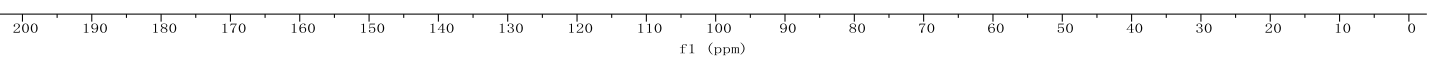<smiles>CC1CCc2ccccc2N1</smiles>

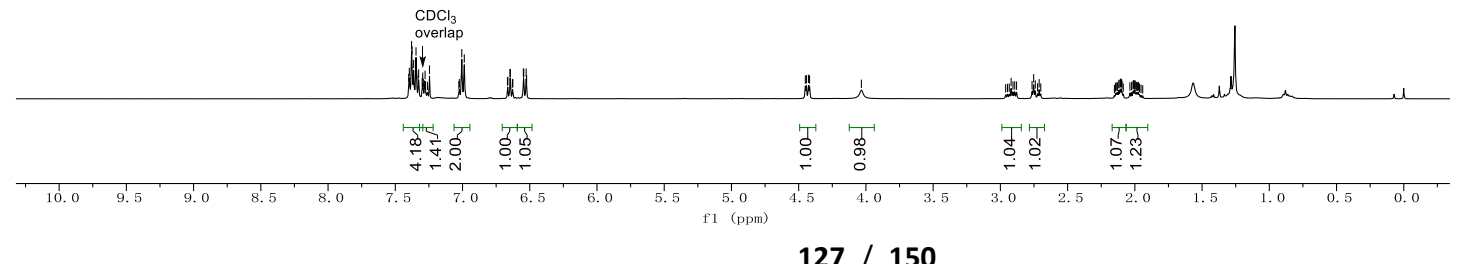

127 / 150 
2cr; ${ }^{13} \mathrm{C} \mathrm{NMR} ; \mathrm{CDCl}_{3} ; 101 \mathrm{MHz}$

至<smiles>C[C@@H]1CCc2ccccc2N1</smiles>

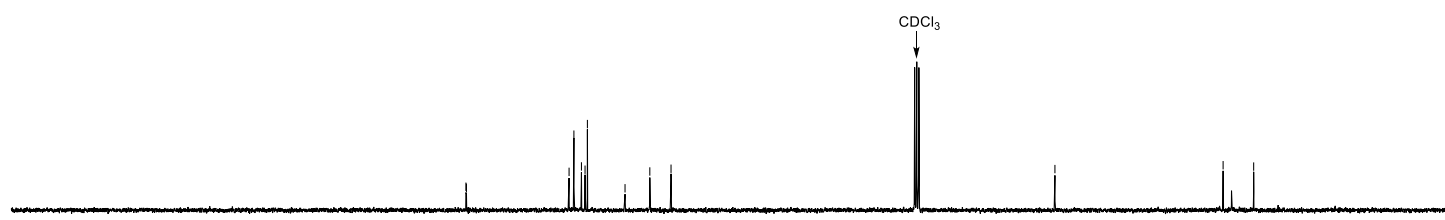

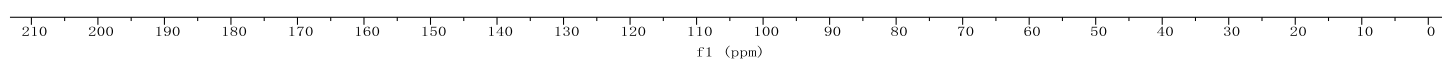
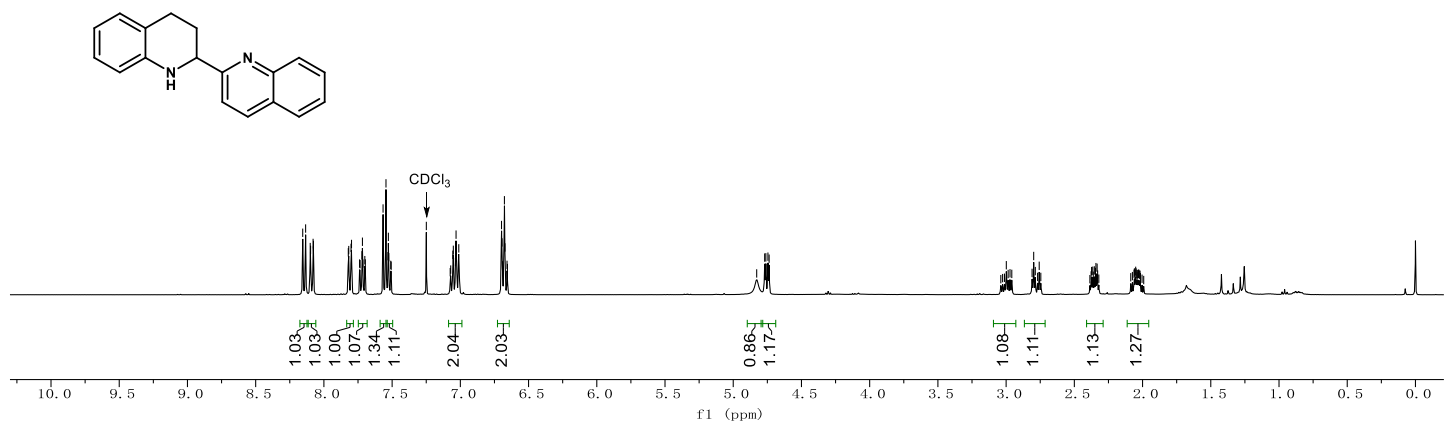
$\mathbf{2 c s} ;{ }^{13} \mathrm{C} \mathrm{NMR} ; \mathrm{CDCl}_{3} ; 101 \mathrm{MHz}$

ll

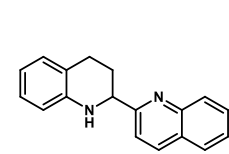

(1)

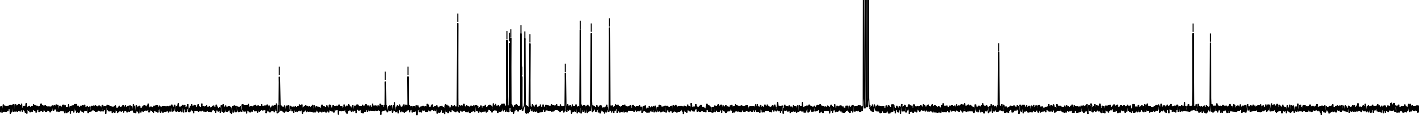

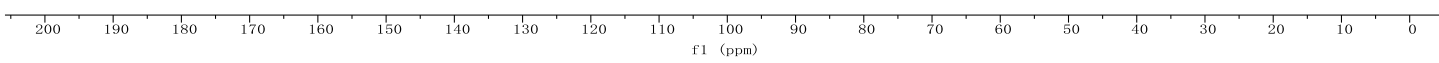

2ct; ${ }^{1} \mathrm{H} \mathrm{NMR} ; \mathrm{CDCl}_{3} ; 400 \mathrm{MHz}$

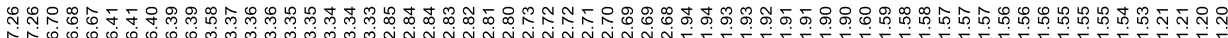

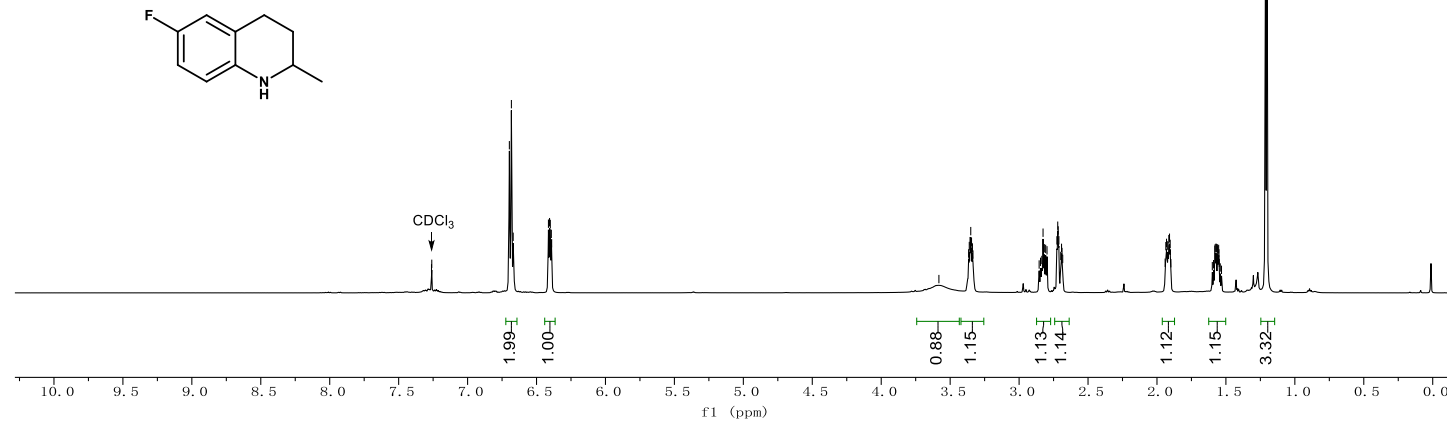

$129 / 150$ 
2ct; ${ }^{13} \mathrm{C}$ NMR; $\mathrm{CDCl}_{3} ; 101 \mathrm{MHz}$
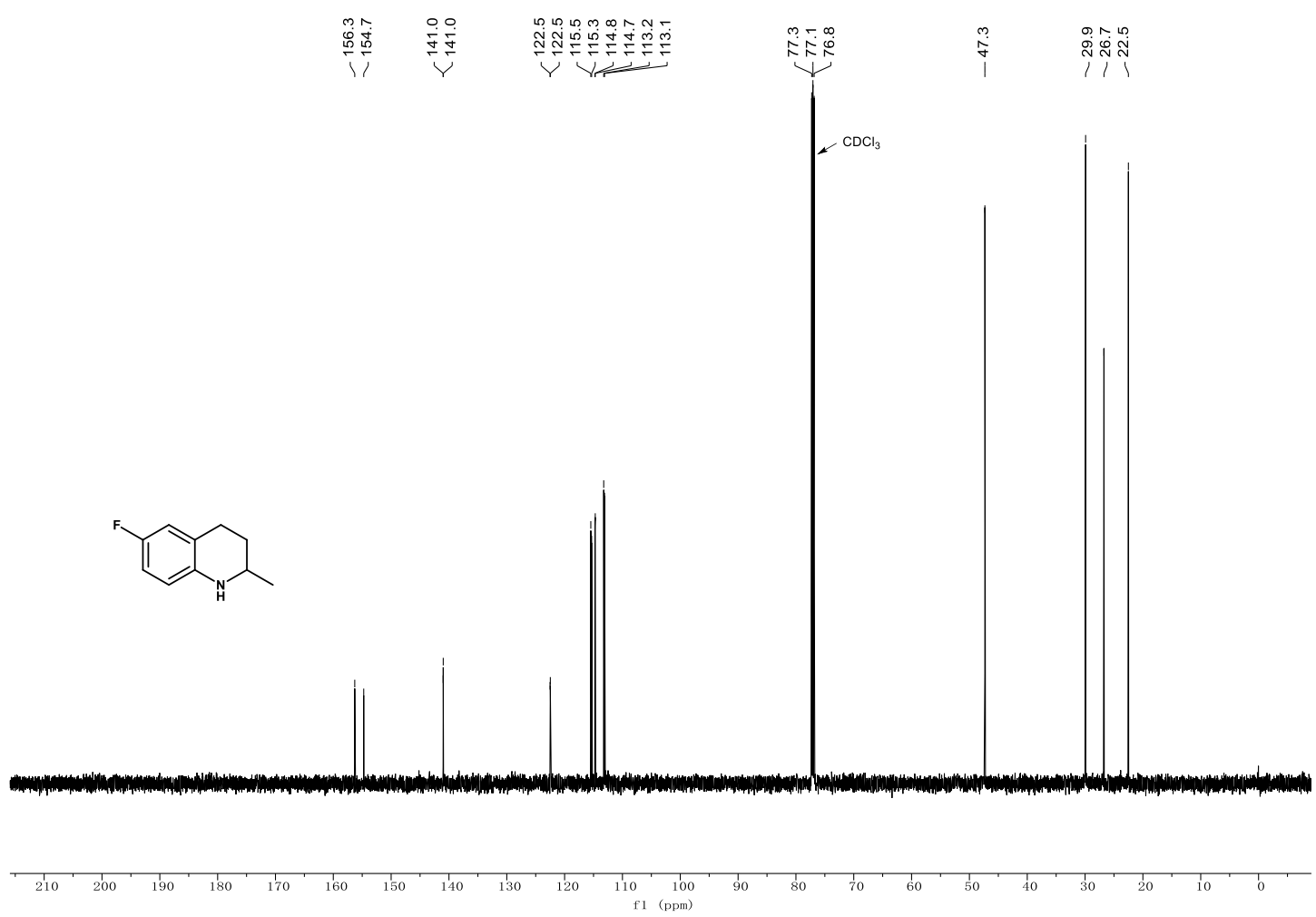

2ct; ${ }^{19} \mathrm{~F}$ NMR; $\mathrm{CDCl}_{3} ; 376 \mathrm{MHz}$
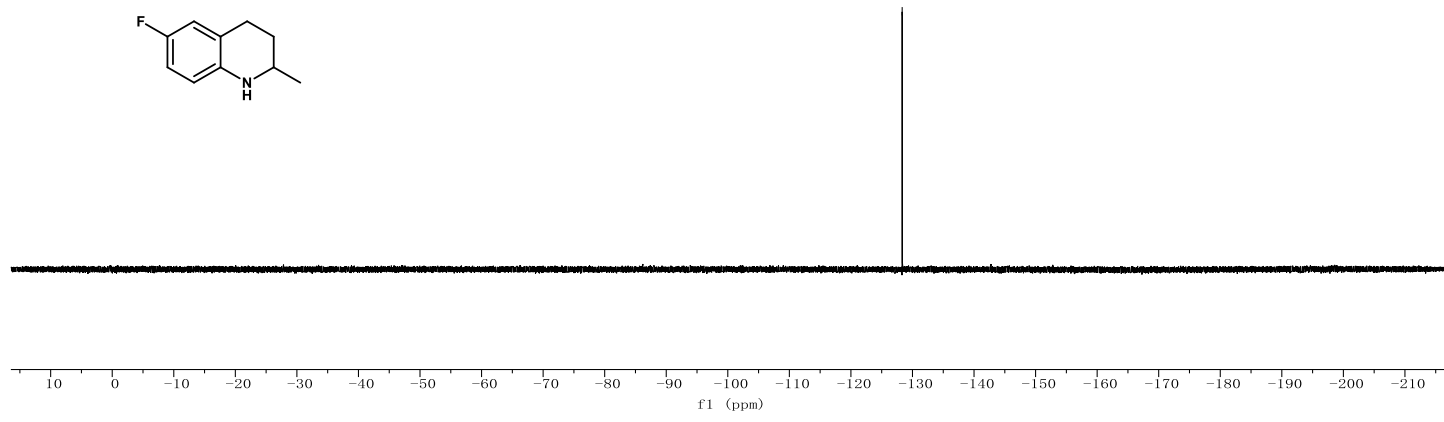
2da; ${ }^{1} \mathrm{H} \mathrm{NMR} ; \mathrm{CDCl}_{3} ; 600 \mathrm{MHz}$

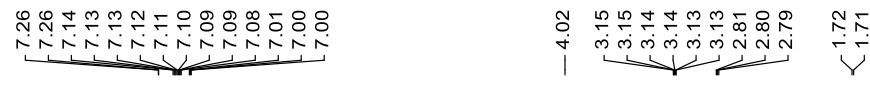

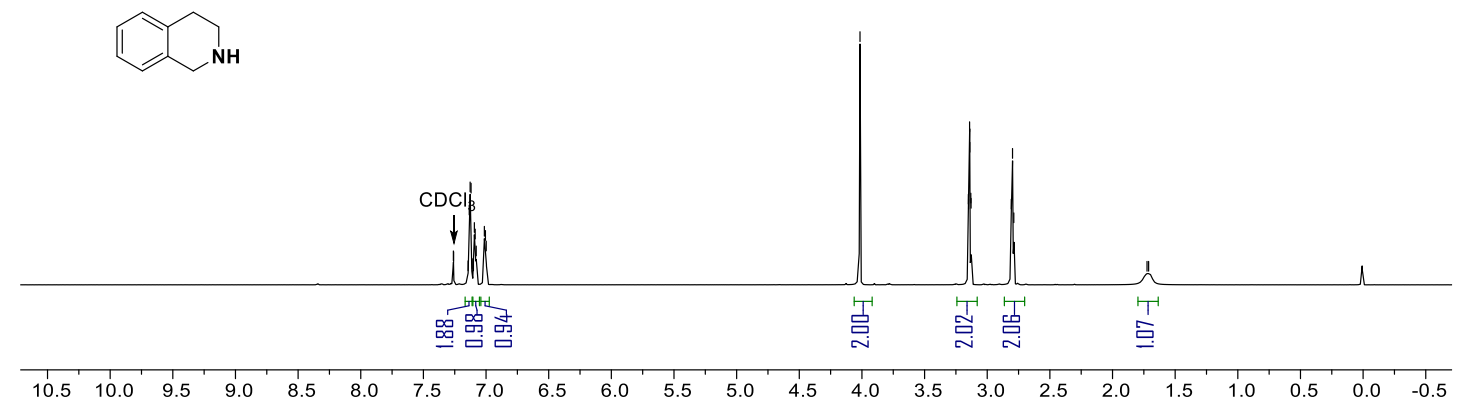

2da; ${ }^{13} \mathrm{C} \mathrm{NMR} ; \mathrm{CDCl}_{3} ; 151 \mathrm{MHz}$

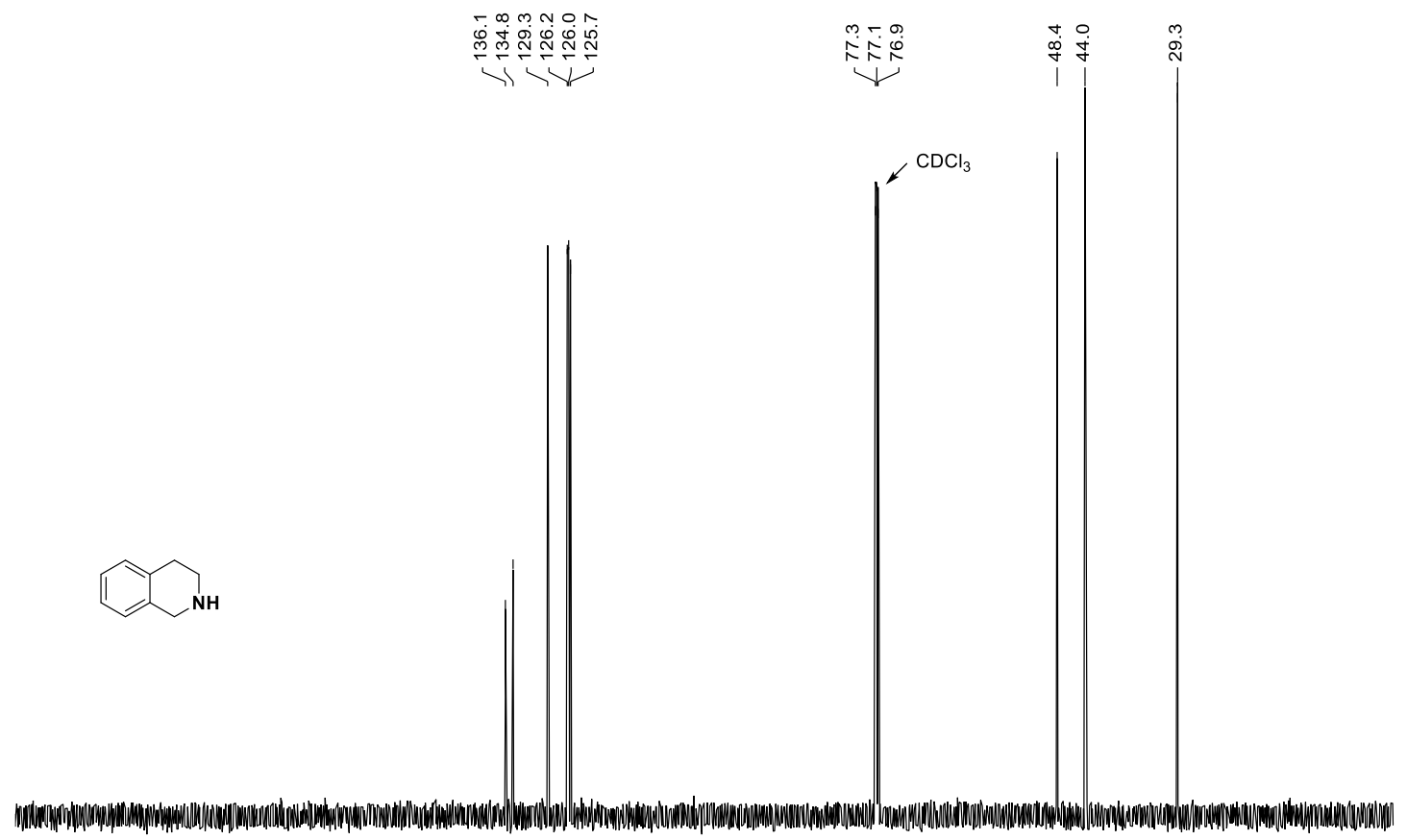

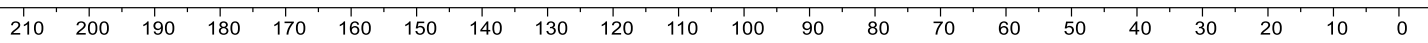

131 / 150 
2db; ${ }^{1} \mathrm{H}$ NMR; $\mathrm{CDCl}_{3} ; 400 \mathrm{MHz}$

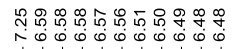

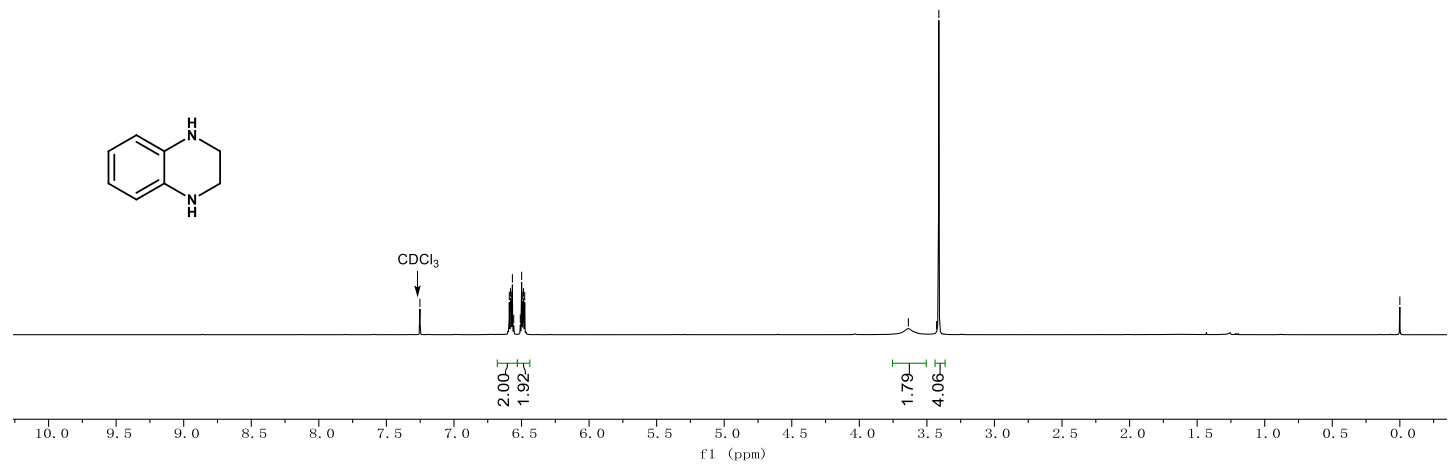

2db; $;{ }^{13} \mathrm{C} \mathrm{NMR} ; \mathrm{CDCl}_{3} ; 101 \mathrm{MHz}$

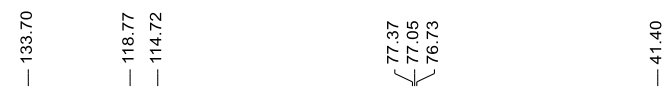

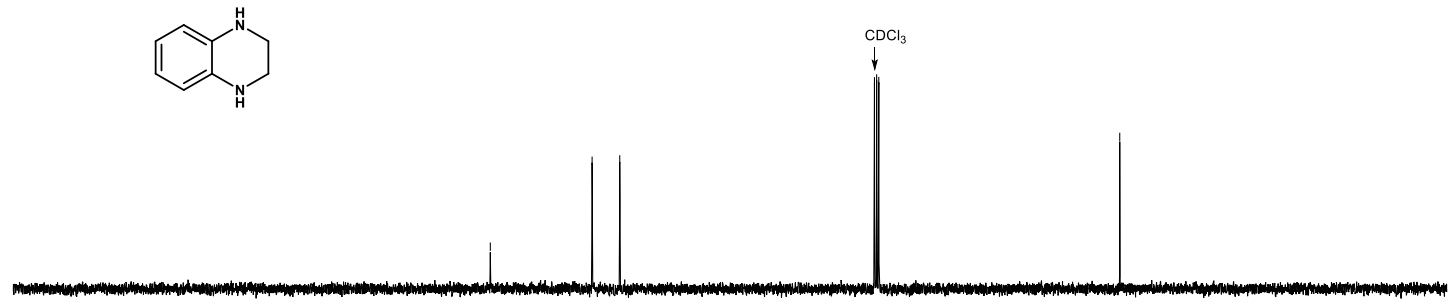

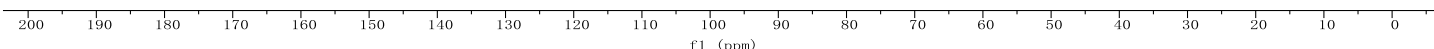
$132 / 150$ 
2dc; ${ }^{1} \mathrm{H} \mathrm{NMR} ; \mathrm{CDCl}_{3} ; 400 \mathrm{MHz}$

i⿱乛龰)

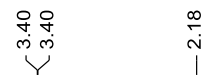

$\stackrel{5}{i}$

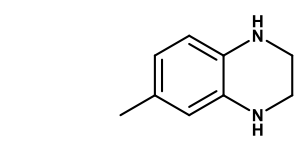

2dc; ${ }^{13} \mathrm{C} \mathrm{NMR} ; \mathrm{CDCl}_{3} ; 101 \mathrm{MHz}$

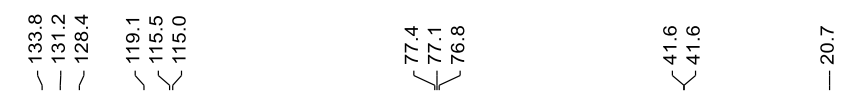

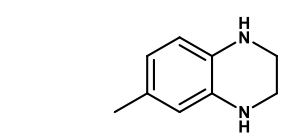

$\mathrm{CDCl}_{3} ; 400 \mathrm{MHz}$ 


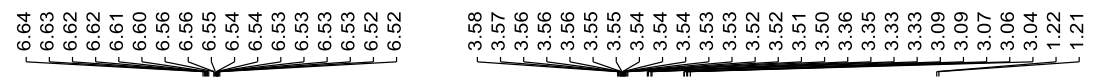
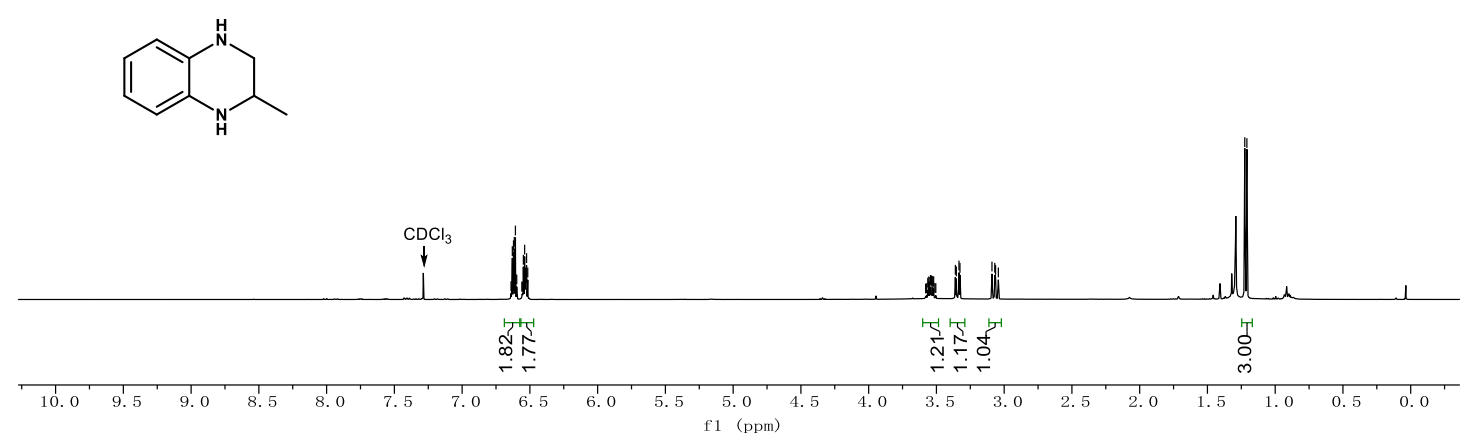

2dd; ${ }^{13} \mathrm{C} \mathrm{NMR} ; \mathrm{CDCl}_{3} ; 101 \mathrm{MHz}$

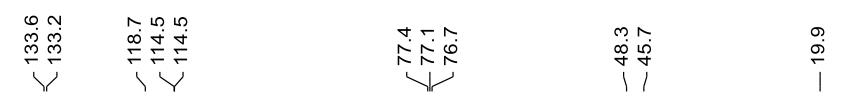<smiles>CC1CNc2ccccc2N1</smiles>

2de; ${ }^{1} \mathrm{H}$ NMR;

$\mathrm{CDCl}_{3} ; 400 \mathrm{MHz}$ 
$\overbrace{}^{n}$

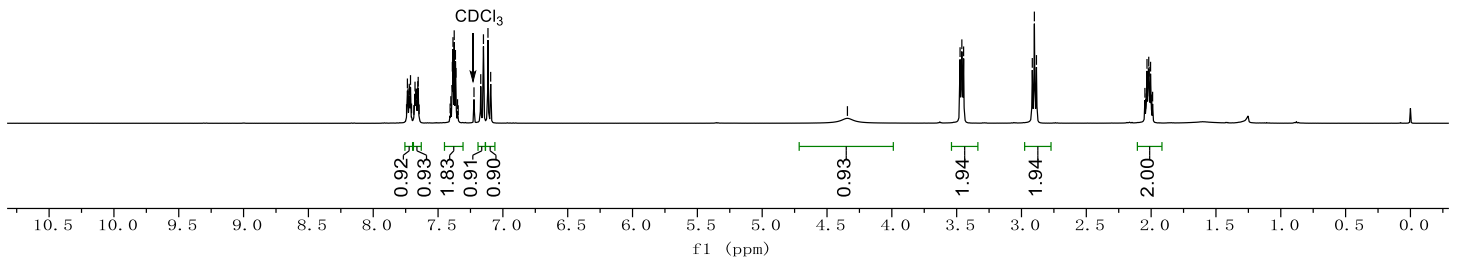

2de; ${ }^{13} \mathrm{C} \mathrm{NMR} ; \mathrm{CDCl}_{3} ; 101 \mathrm{MHz}$

$\underbrace{\dot{m}}$
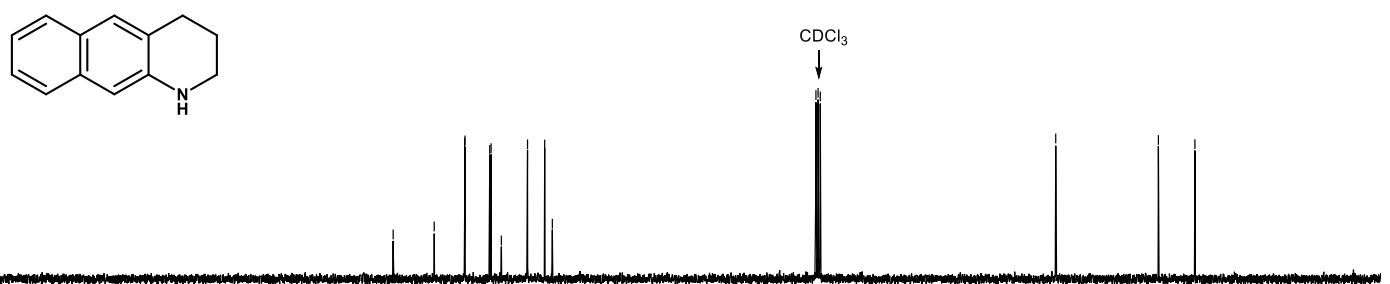

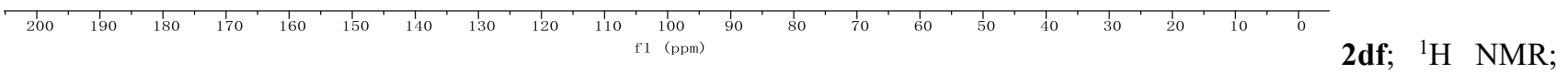

$\mathrm{CDCl}_{3} ; 400 \mathrm{MHz}$ 

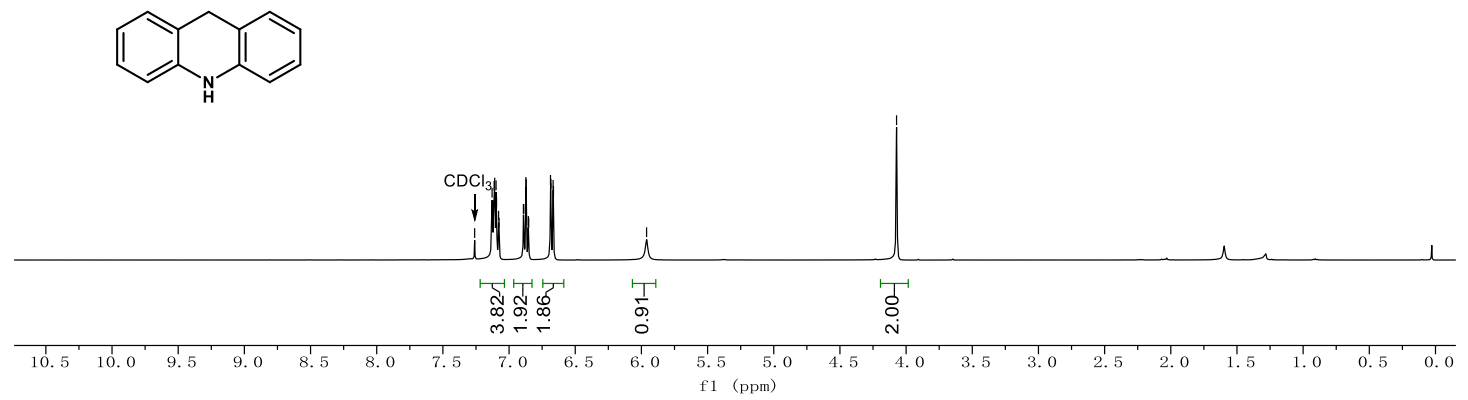

2df; ${ }^{13} \mathrm{C} \mathrm{NMR} ; \mathrm{CDCl}_{3} ; 101 \mathrm{MHz}$

竞
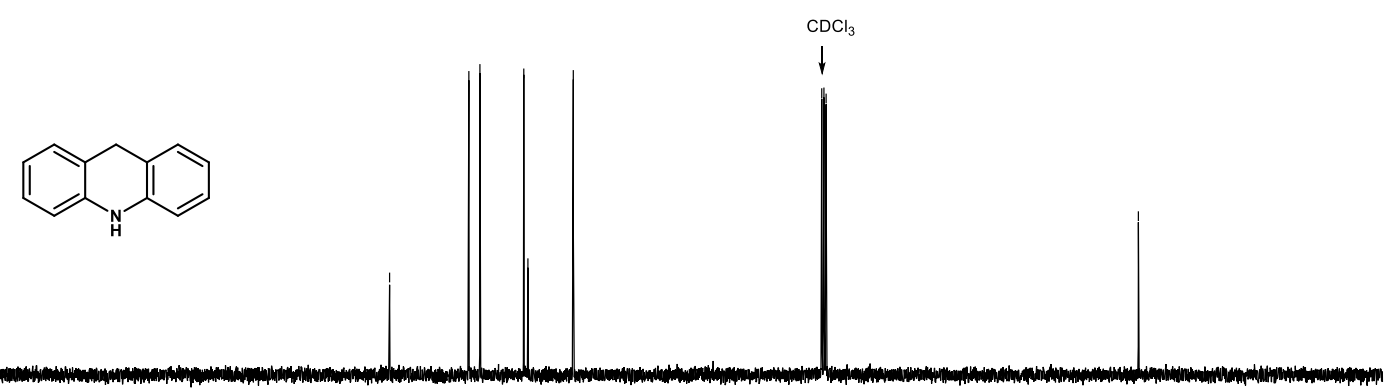

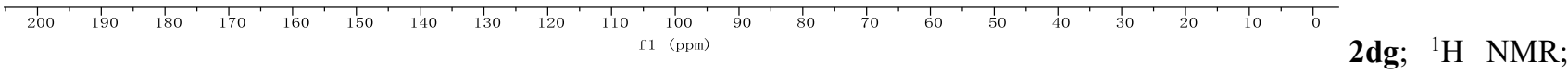

$\mathrm{CDCl}_{3} ; 400 \mathrm{MHz}$ 

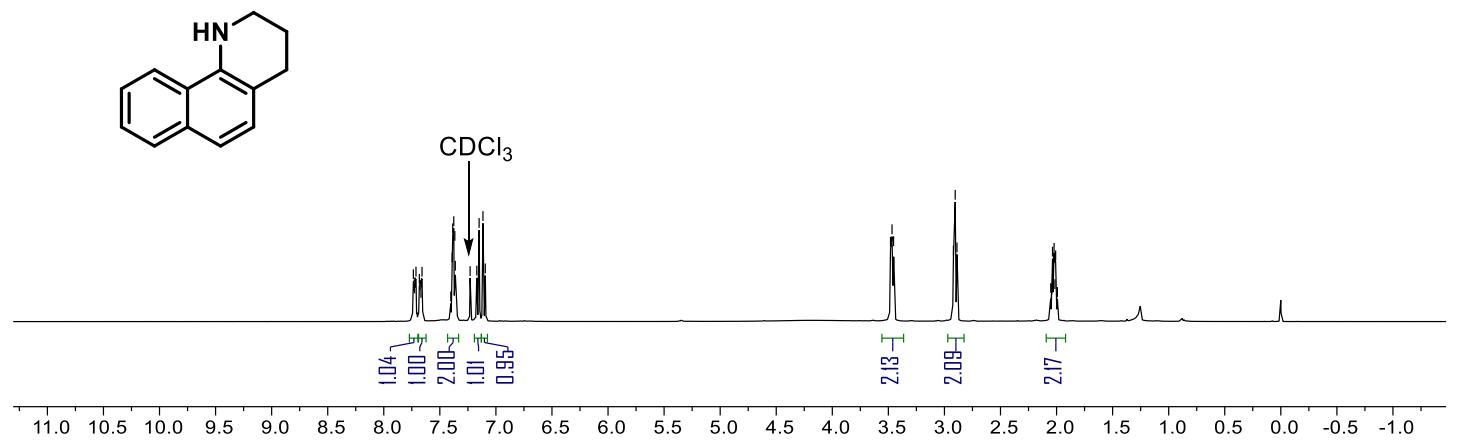

2dg; ${ }^{13} \mathrm{C} \mathrm{NMR} ; \mathrm{CDCl}_{3} ; 101 \mathrm{MHz}$

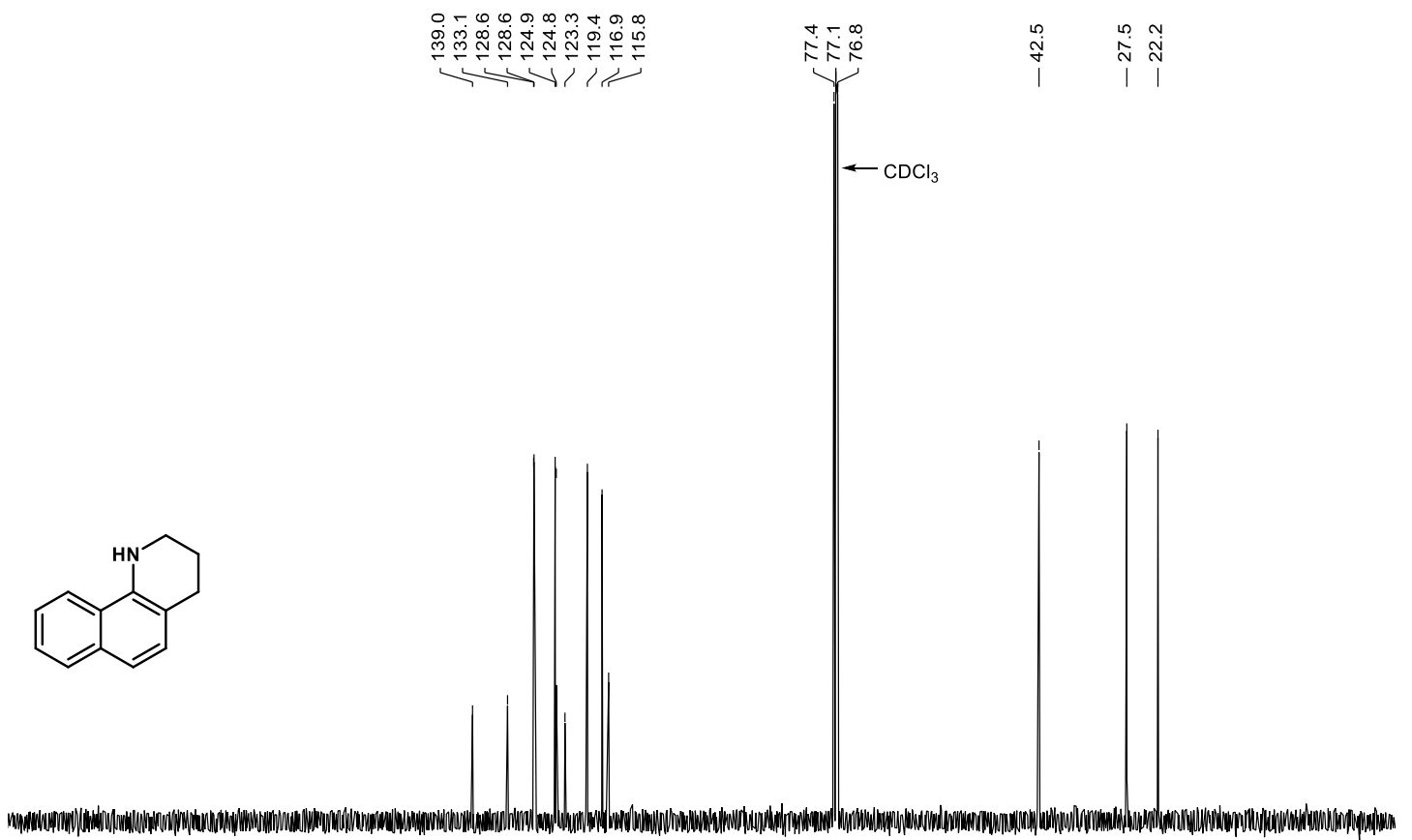

$\begin{array}{lllllllllllllllllllllll}210 & 200 & 190 & 180 & 170 & 160 & 150 & 140 & 130 & 120 & 110 & 100 & 90 & 80 & 70 & 60 & 50 & 40 & 30 & 20 & 10 & 0 & -10\end{array}$ 2dh; ${ }^{1} \mathrm{H}$ NMR; $\mathrm{CDCl}_{3} ; 400 \mathrm{MHz}$ 

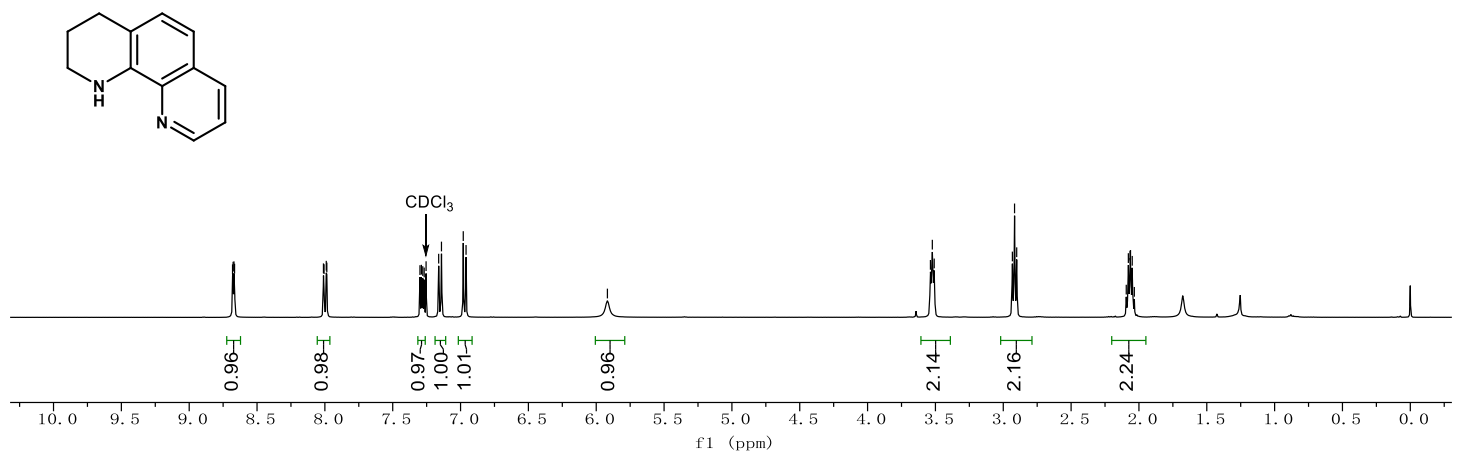

2dh; $;{ }^{13} \mathrm{C} \mathrm{NMR} ; \mathrm{CDCl}_{3} ; 101 \mathrm{MHz}$
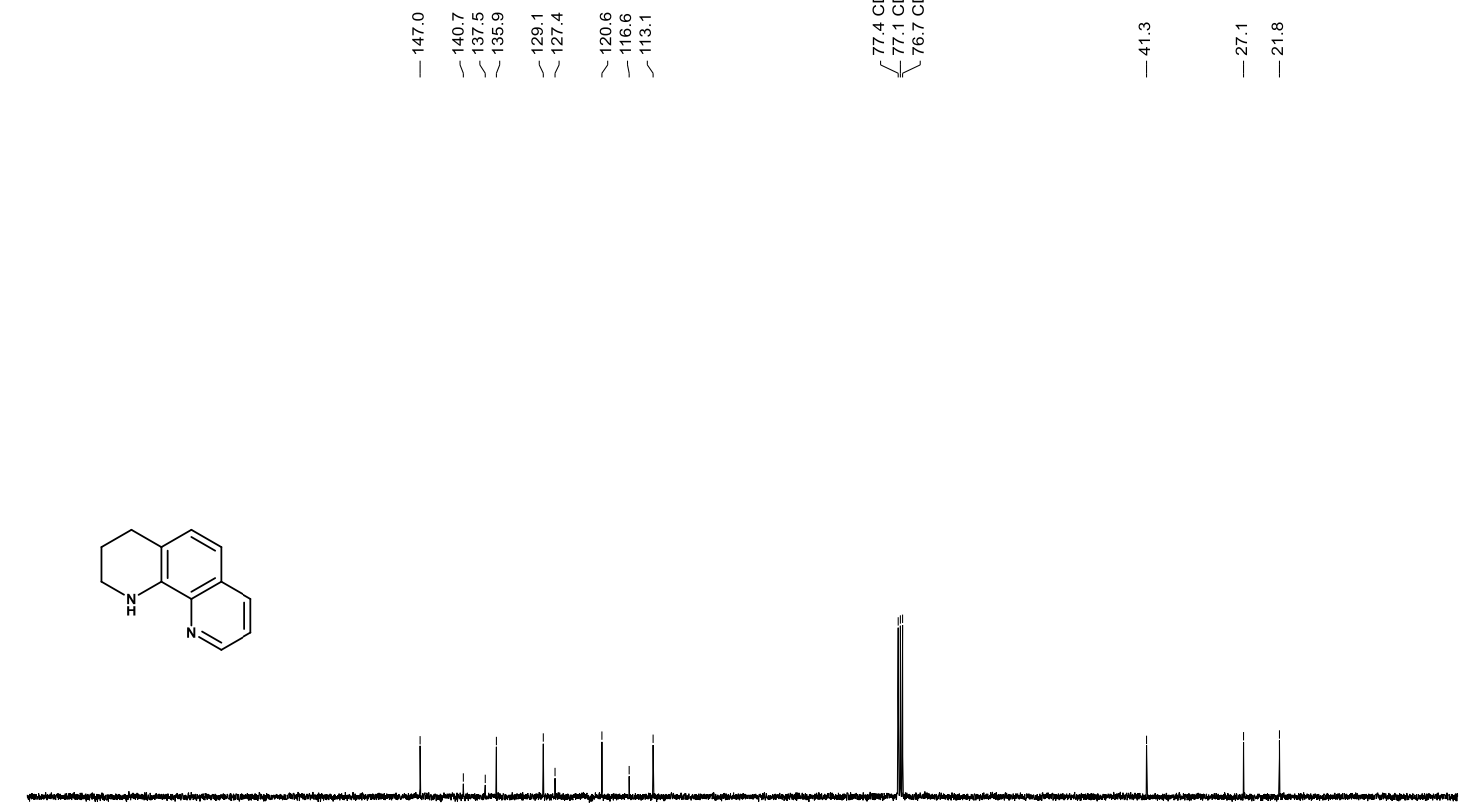

\section{i}

Th

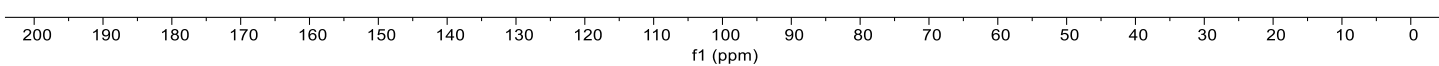

2di; ${ }^{1} \mathrm{H}$ NMR; $\mathrm{CDCl}_{3} ; 400 \mathrm{MHz}$ 


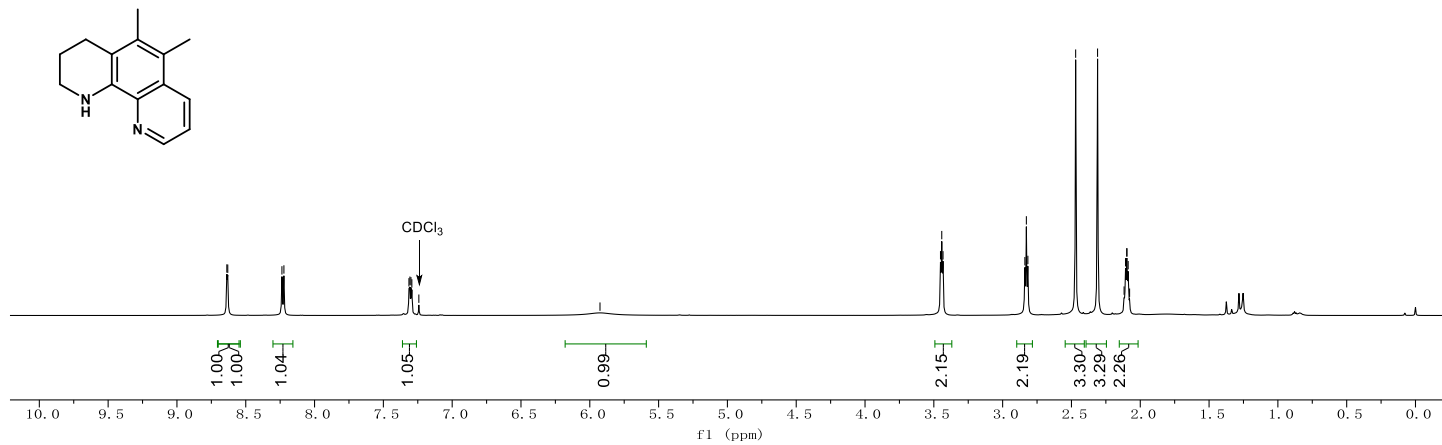

2di; ${ }^{13} \mathrm{C} \mathrm{NMR} ; \mathrm{CDCl}_{3} ; 101 \mathrm{MHz}$
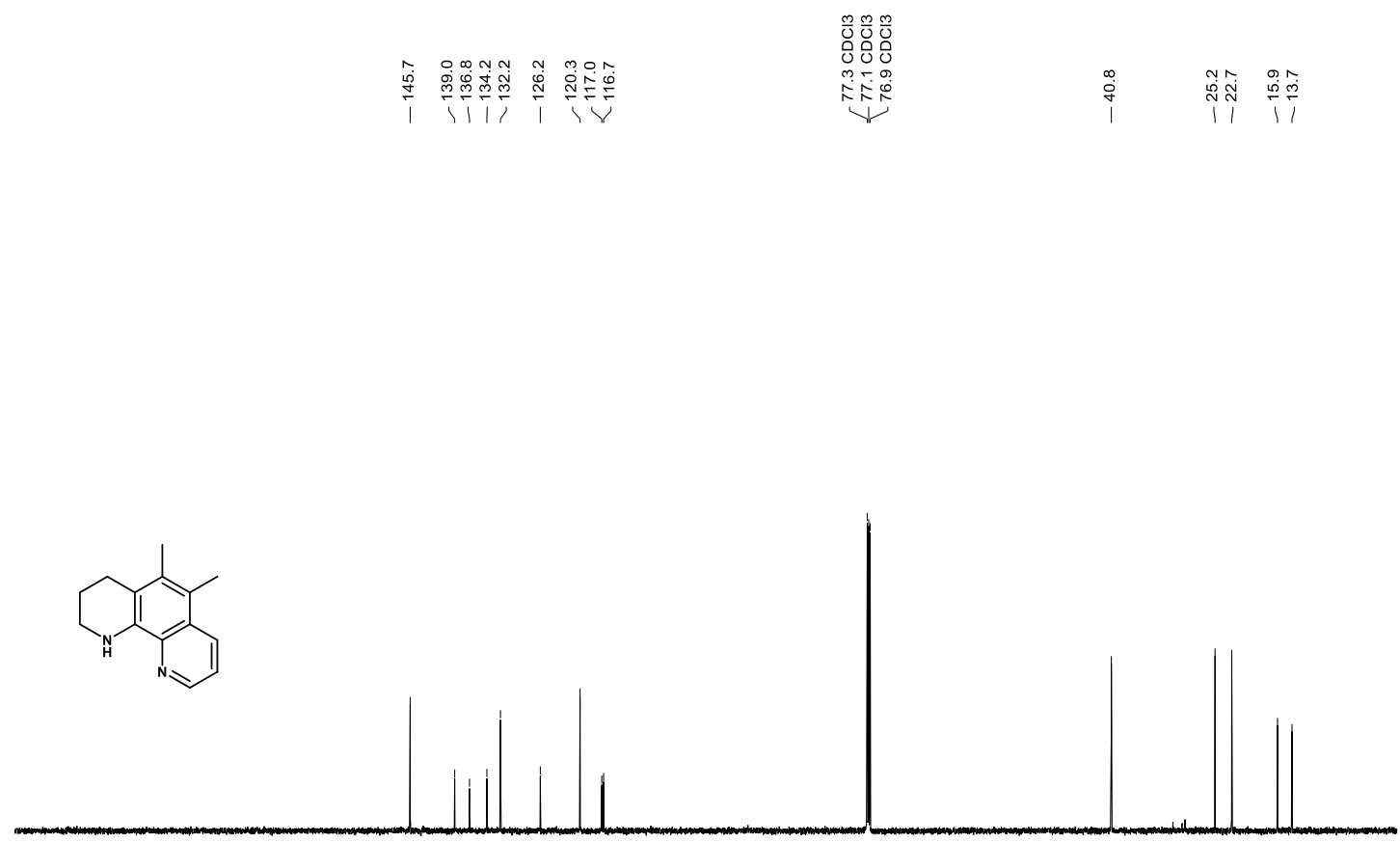

2dj; ${ }^{1} \mathrm{H}$ NMR; $\mathrm{CDCl}_{3} ; 400 \mathrm{MHz}$ 


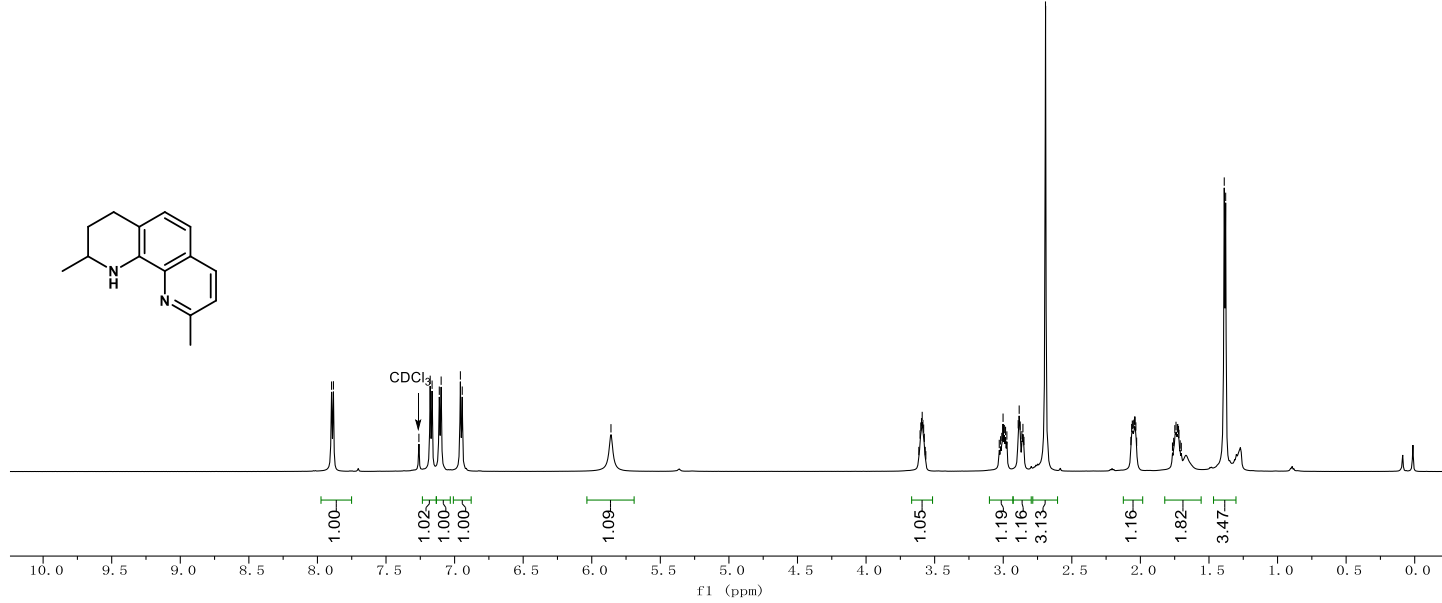

2dk; ${ }^{13} \mathrm{C} \mathrm{NMR} ; \mathrm{CDCl}_{3} ; 101 \mathrm{MHz}$

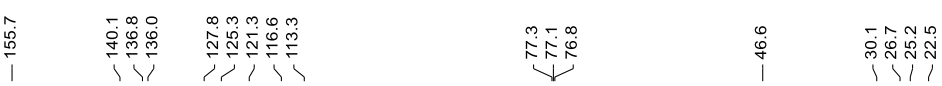

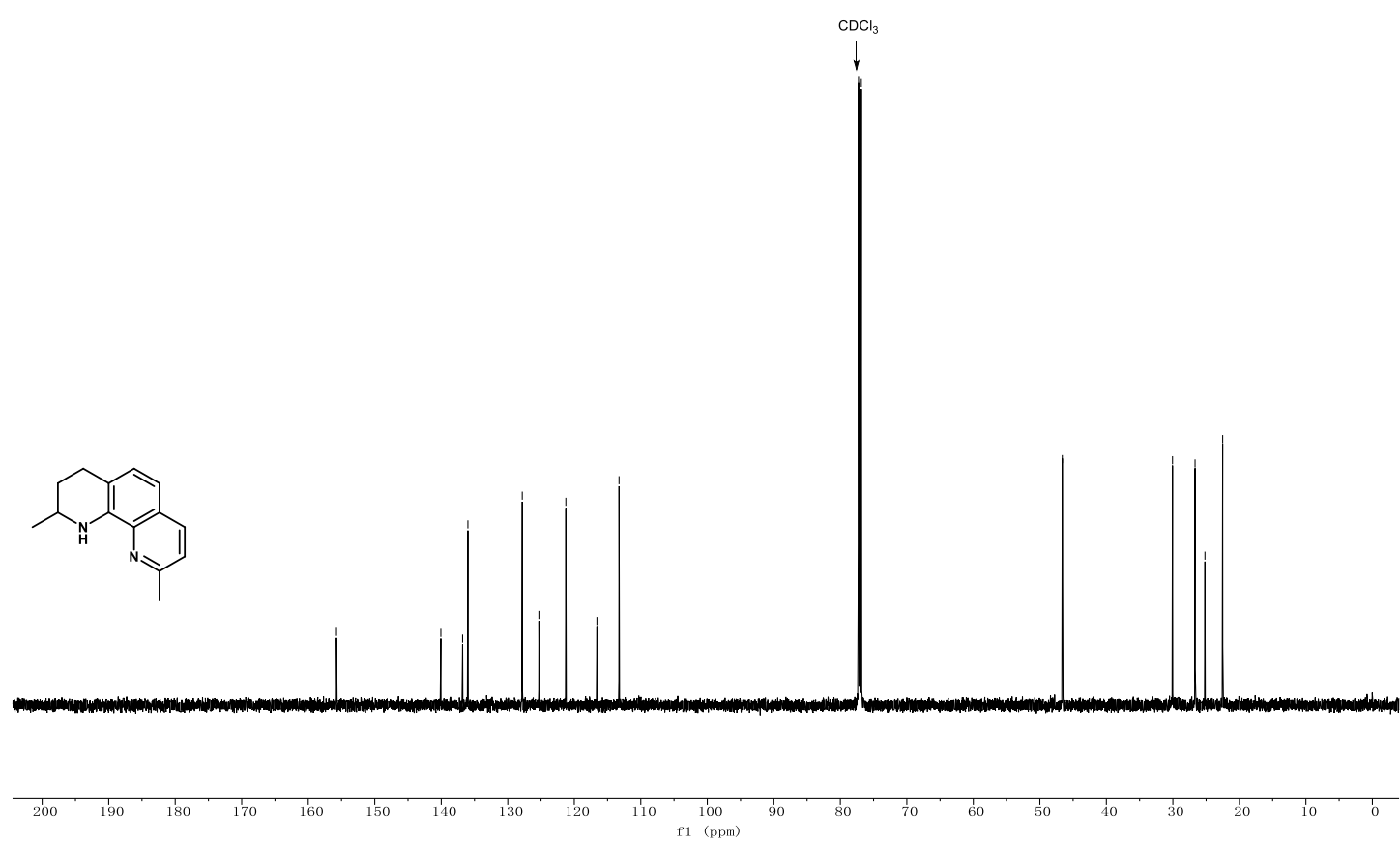

2ea; ${ }^{1} \mathrm{H}$ NMR; $\mathrm{CDCl}_{3} ; 400 \mathrm{MHz}$ 

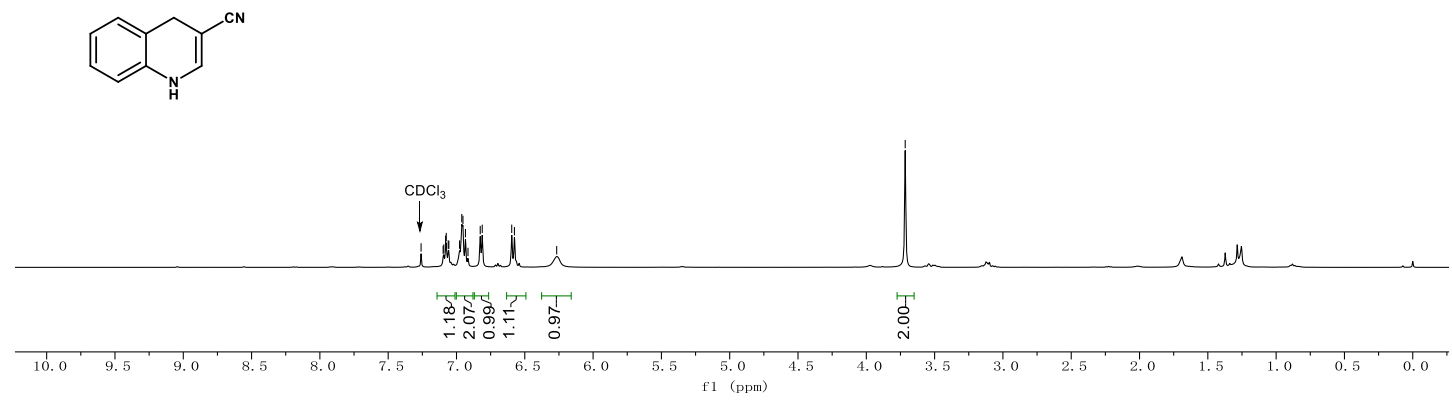

2ea; ${ }^{13} \mathrm{C}$ NMR; $\mathrm{CDCl}_{3} ; 101 \mathrm{MHz}$

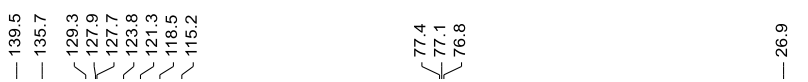

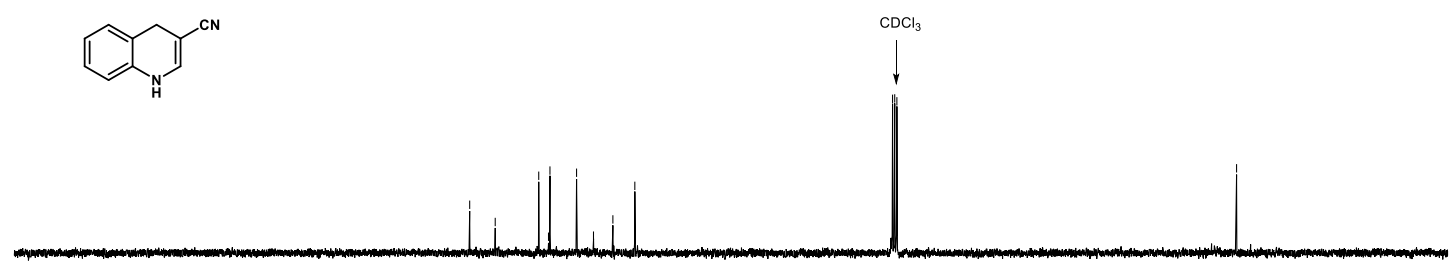

2eb; ${ }^{1} \mathrm{H} \mathrm{NMR} ; \mathrm{CDCl}_{3} ; 400 \mathrm{MHz}$ 

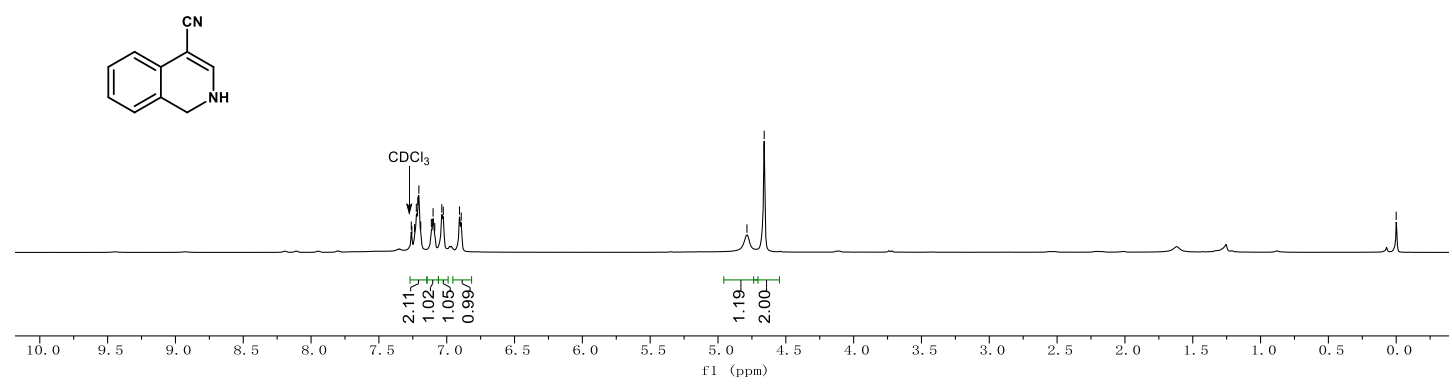

2eb; ${ }^{13} \mathrm{C}$ NMR; $\mathrm{CDCl}_{3} ; 101 \mathrm{MHz}$
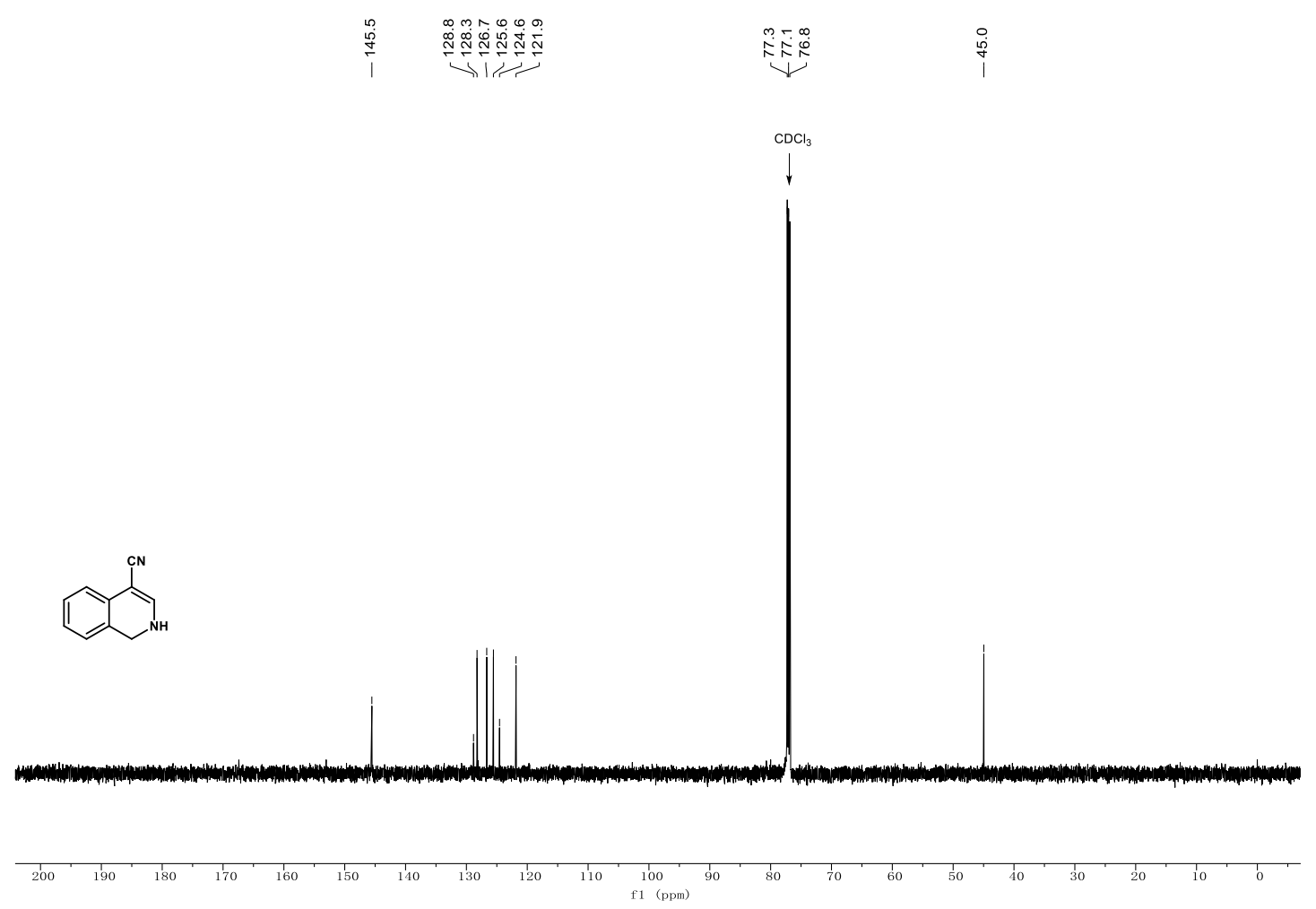

2ec; ${ }^{1} \mathrm{H}$ NMR; $\mathrm{CDCl}_{3} ; 400 \mathrm{MHz}$ 

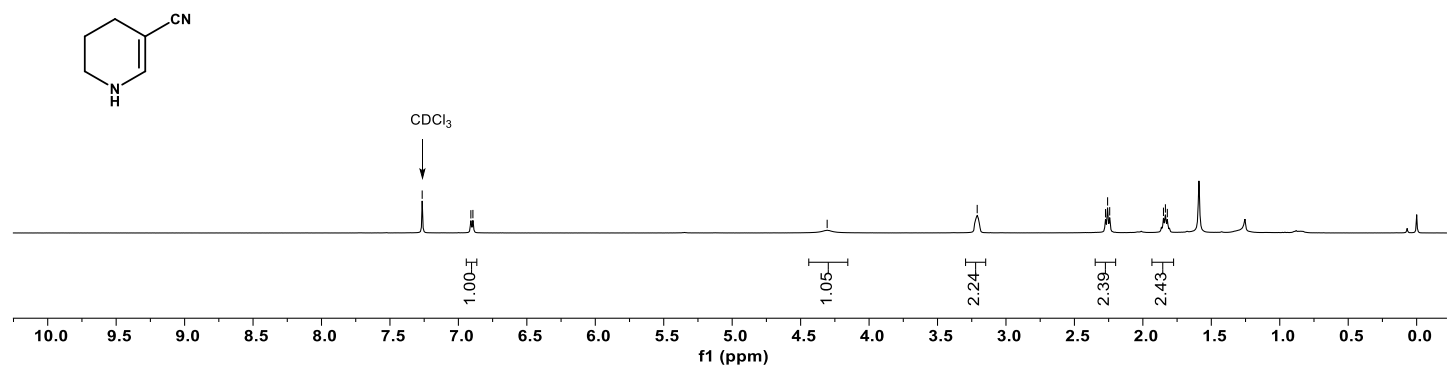

2ec; ${ }^{13} \mathrm{C}$ NMR; $\mathrm{CDCl}_{3} ; 101 \mathrm{MHz}$

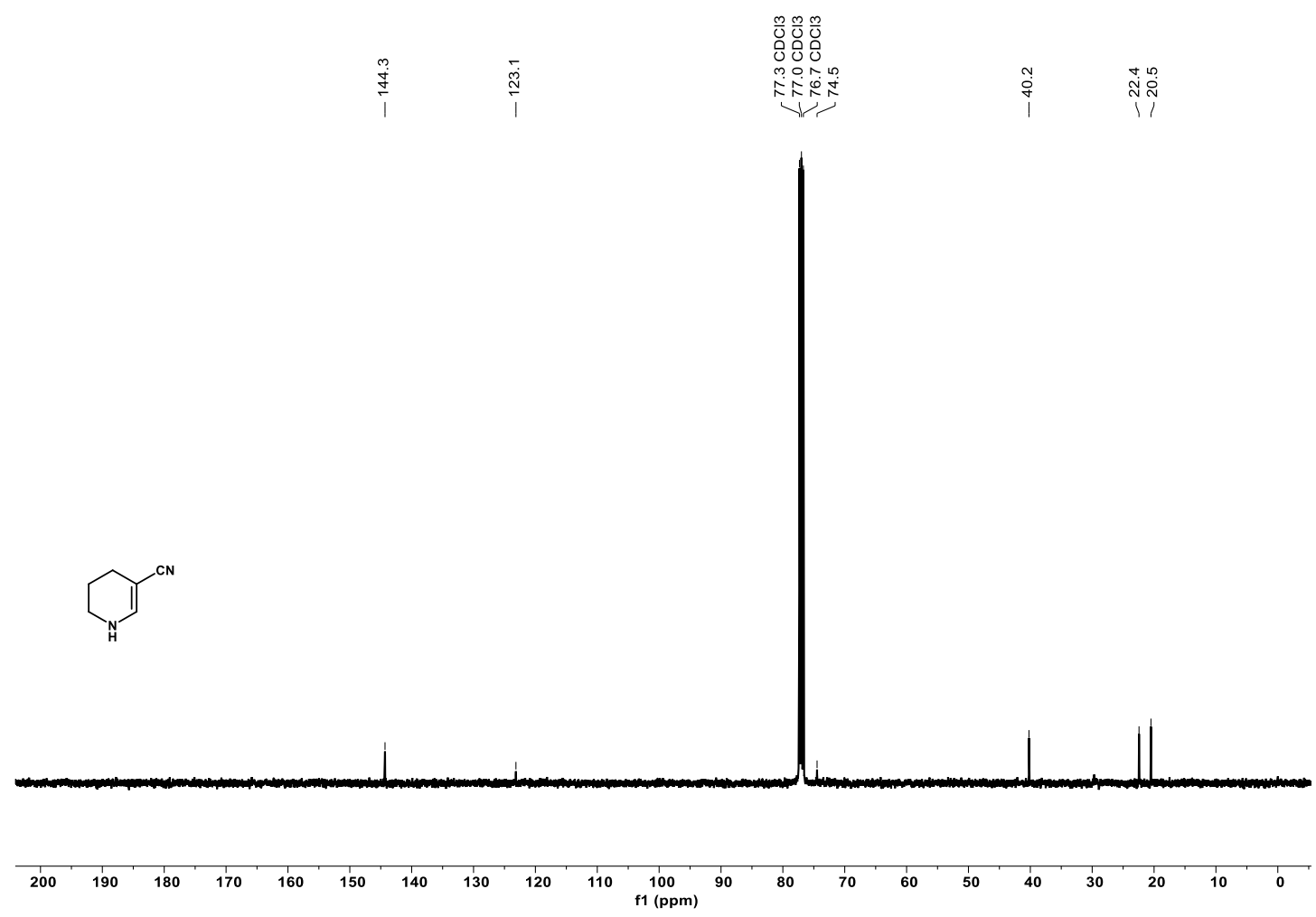

$143 / 150$ 
2ed; ${ }^{1} \mathrm{H} \mathrm{NMR} ; \mathrm{CDCl}_{3} ; 400 \mathrm{MHz}$
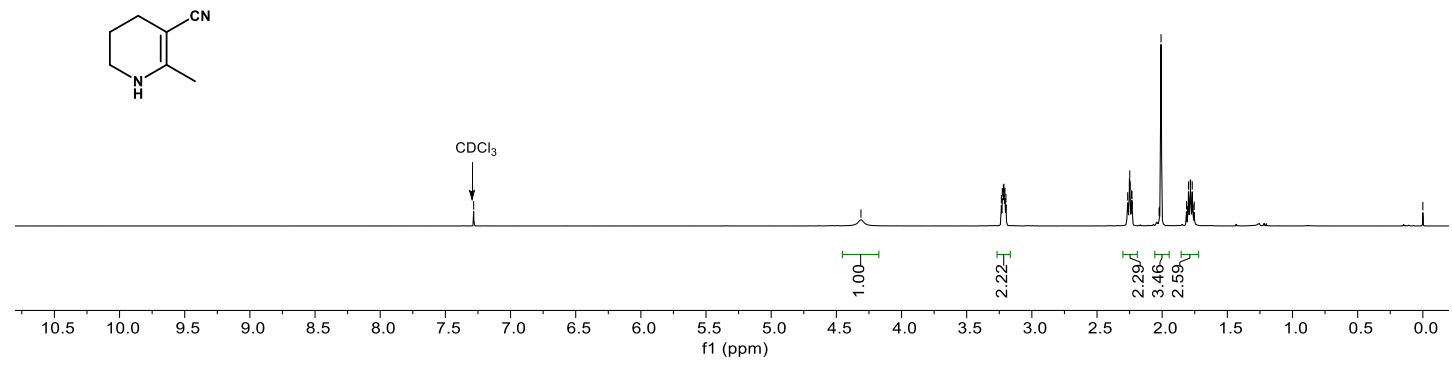

2ed; ${ }^{13} \mathrm{C}$ NMR; $\mathrm{CDCl}_{3} ; 101 \mathrm{MHz}$

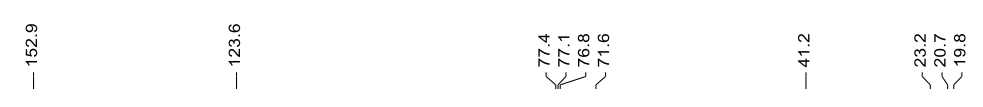

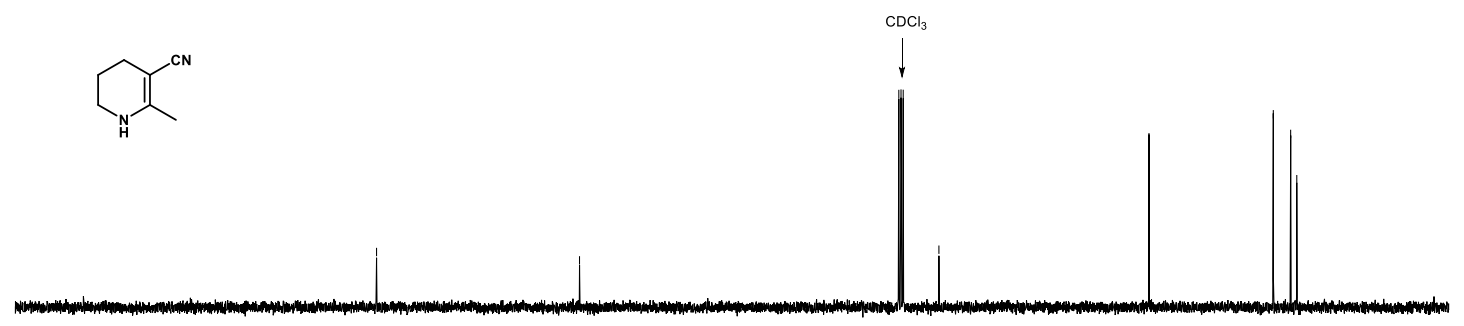

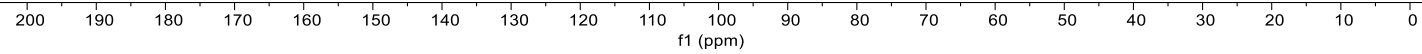


2ee; ${ }^{1} \mathrm{H} \mathrm{NMR} ; \mathrm{CDCl}_{3} ; 400 \mathrm{MHz}$

i

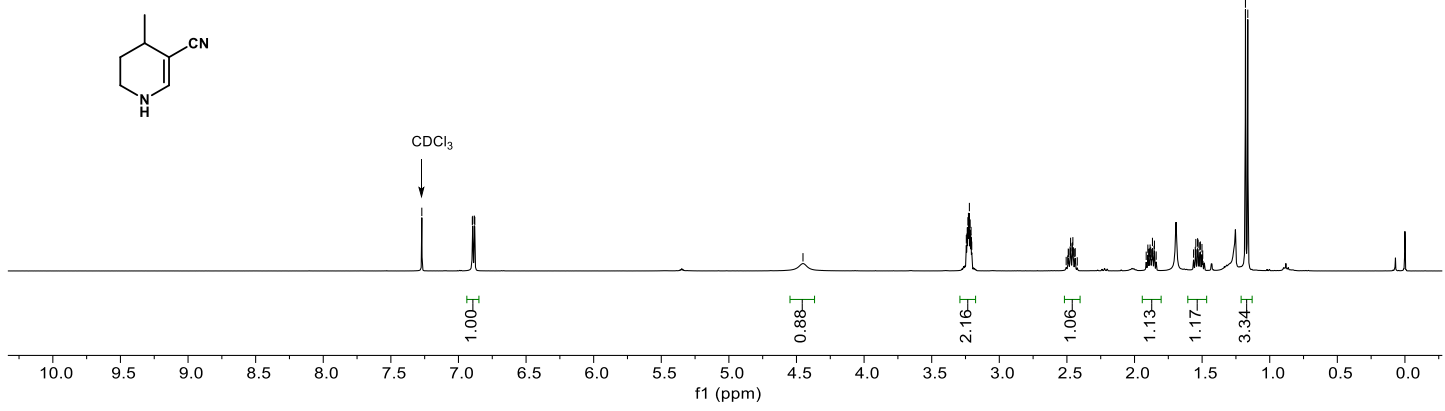

2ee; ${ }^{13} \mathrm{C} \mathrm{NMR} ; \mathrm{CDCl}_{3} ; 101 \mathrm{MHz}$

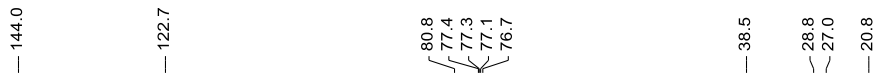

$\overbrace{}^{\mathrm{CN}}$
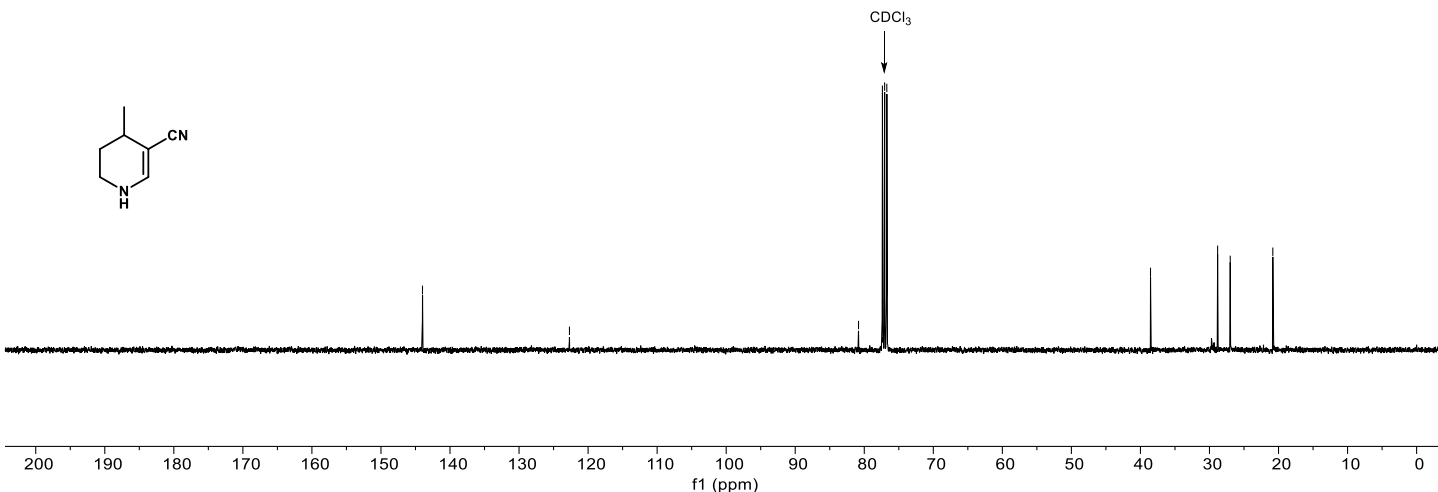

2ef; ${ }^{1} \mathrm{H} \mathrm{NMR} ; \mathrm{CDCl}_{3} ; 600 \mathrm{MHz}$ 


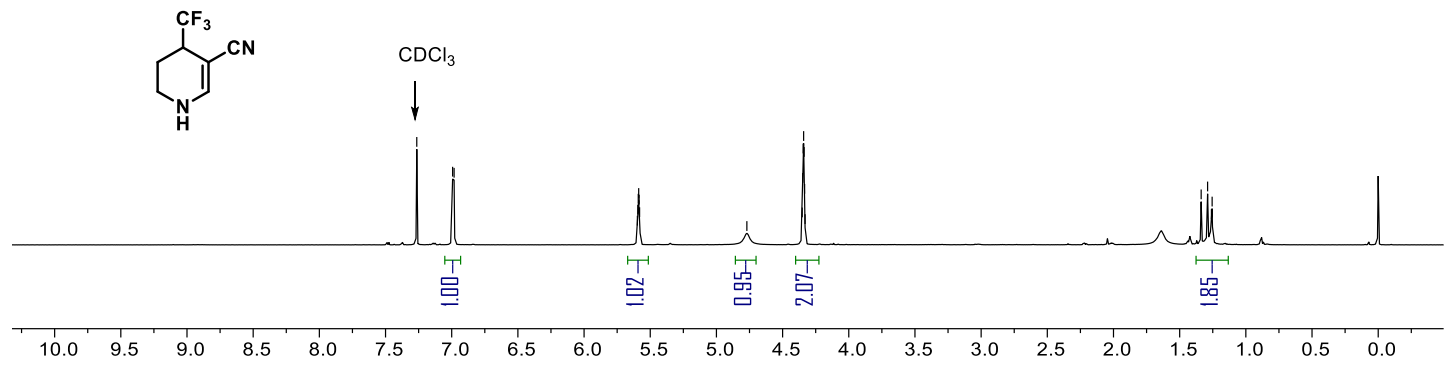

2ef; ${ }^{13} \mathrm{C} \mathrm{NMR} ; \mathrm{CDCl}_{3} ; 151 \mathrm{MHz}$

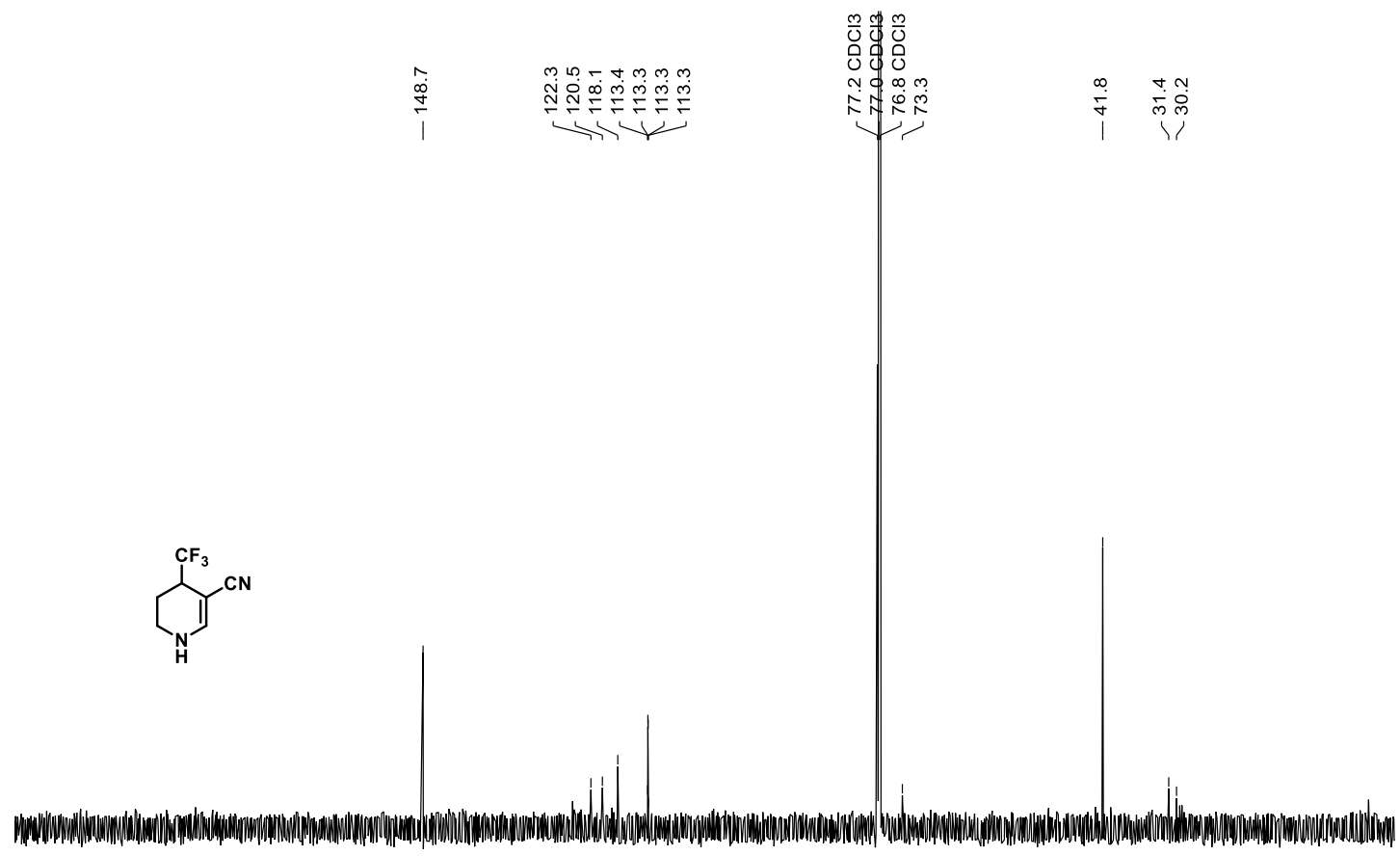

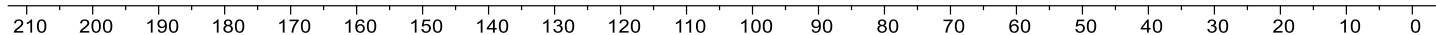

2ef; ${ }^{19} \mathrm{~F} \mathrm{NMR} ; \mathrm{CDCl}_{3} ; 565 \mathrm{MHz}$ 


$$
\stackrel{\substack{? \\ \mathfrak{n}}}{i}
$$

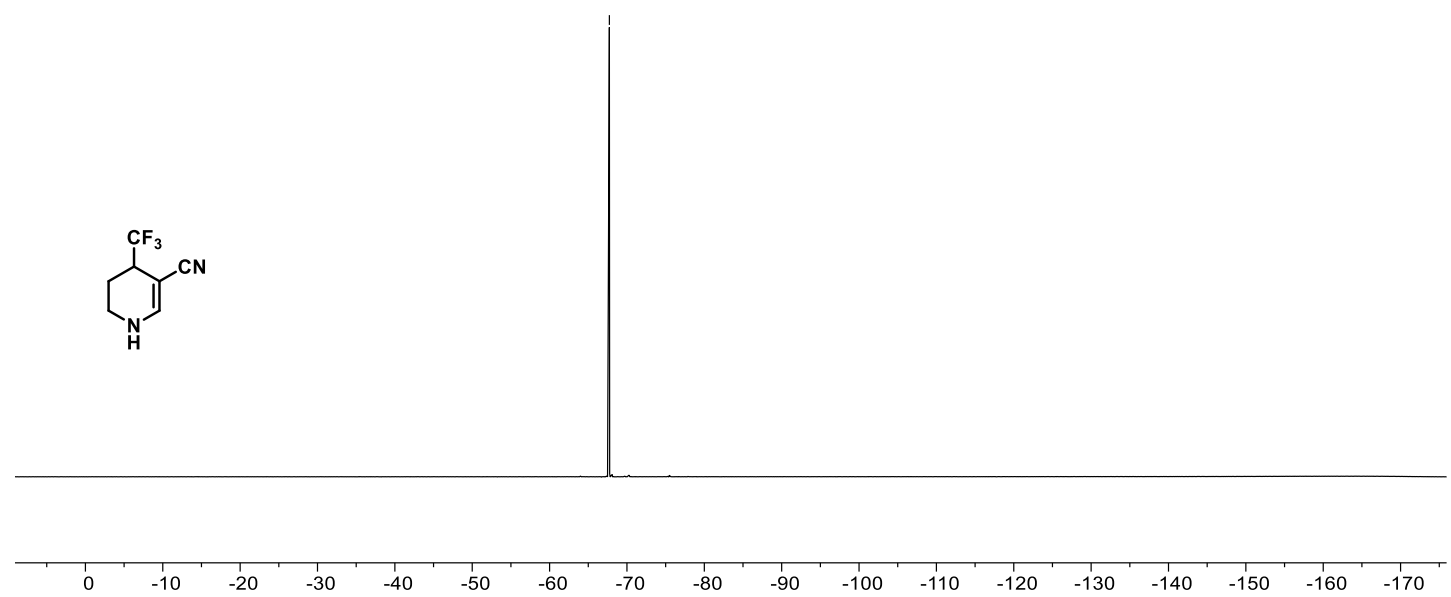




\section{Reference}

[1] (a) Wiedner, E. S.; Roberts, J. A. S.; Dougherty, W. G.; Kassel, W. S.; DuBois, D. L.; Bullock, R. M. Synthesis and Electrochemical Studies of Cobalt(III) Monohydride Complexes Containing Pendant Amines. Inorg. Chem. 2013, 52, 9975-9988; (b) Burgess, S. A.; Grubel, K.; Appel, A. M.; Wiedner, E. S.; Linehan, J. C. Hydrogenation of $\mathrm{CO}_{2}$ at Room Temperature and Low Pressure with a Cobalt Tetraphosphine Catalyst. Inorg. Chem. 2017, 56, 8580-8589.

[2] Kilgore, U. J.; Stewart, M. P.; Helm, M. L.; Dougherty, W. G.; Kassel, W. S.; DuBois, M. R.; DuBois, D. L.; Bullock, R. M., Studies of a Series of $[\mathrm{Ni}(\mathrm{PR} 2 \mathrm{NPh} 2) 2(\mathrm{CH} 3 \mathrm{CN})] 2+$ Complexes as Electrocatalysts for H2 Production: Substituent Variation at the Phosphorus Atom of the P2N2 Ligand. Inorg. Chem. 2011, 50, 10908-10918.

[3] Malkov, A. V.; Gouriou, L.; Lloyd-Jones, G. C.; Starý,V.Langer, I.; Spoor, P.; Vinader, V.; Kocovský, P.; Chem. Eur. J. 2006, $12,6910$.

[4] Frisch, M. J.; Trucks, G. W.; Schlegel, H. B.; Scuseria, G. E.; Robb, M. A.; Cheeseman, J. R.; Scalmani, G.; Barone, V.; Mennucci, B.; Petersson, G. A.; Nakatsuji, H.; Caricato, M.; Li, X.; Hratchian, H. P.; Izmaylov, A. F.; Bloino, J.; Zheng, G.; Sonnenberg, J. L.; Hada, M. E., M.; ; Toyota, K. F., R.; ; Hasegawa, J.; Ishida, M.; Nakajima, T.; Honda, Y.; Kitao, O.; Nakai, H.; Vreven, T.; Montgomery, J., J. A.;; Peralta, J. E.; Ogliaro, F.; Bearpark, M.; Heyd, J. J.; Brothers, E.; Kudin, K. N.; Staroverov, V. N.; Kobayashi, R.; Normand, J.; Raghavachari, K.; Rendell, A.; Burant, J. C.; Iyengar, S. S.; Tomasi, J.; Cossi, M.; Rega, N.; Millam, N. J.; Klene, M.; Knox, J. E.; Cross, J. B.; Bakken, V.; Adamo, C.; Jaramillo, J.; Gomperts, R.; Stratmann, R. E.; Yazyev, O.; Austin, A. J.; Cammi, R.; Pomelli, C.; Ochterski, J. W.; Martin, R. L.; Morokuma, K.; Zakrzewski, V. G.; Voth, G. A.; Salvador, P.; Dannenberg, J. J.; Dapprich, S.; Daniels, A. D.; Farkas, Ö.; Foresman, J. B.; Ortiz, J. V.; Cioslowski, J.; Fox, D. J. Gaussian 09, Gaussian, Inc., Wallingford CT, 2009.

[5] (a) Chai, J.-D.; Head-Gordon, M. Systematic optimization of long-range corrected hybrid density functionals. J. Chem. Phys. 2008, 128, 084106. (b) Chai, J.-D.; Head-Gordon, M. Long-range corrected hybrid density functionals with damped atom-atom dispersion corrections. Phys. Chem. Chem. Phys. 2008, 10, 6615-6620.

[6] Dolg, M.; Wedig, U.; Stoll, H.; Preuss, H. Energy - adjusted ab initio pseudopotentials for the first row transition elements. J. Chem. Phys. 1987, 86, 866-872.

[7] (a) Hariharan, P. C.; Pople, J. A. The influence of polarization functions on molecular orbital hydrogenation energies. Theor. Chem. Acc. 1973, 28, 213-222. (b) Hehre, W. J.; Ditchfield, R.; Pople, J. A. Self-consistent molecular orbital methods. XII. Further extensions of Gaussian-type basis sets for use in molecular orbital studies of organic molecules. J. Chem. Phys. 1972, 56, 2257-2261. (c) Clark, T.; Chandrasekhar, J.; Spitznagel, G. W.; Schleyer, P. V. R. Efficient diffuse function-augmented basis sets for anion calculations. III. The $3-21+\mathrm{G}$ basis set for first-row elements, Li-F. J. Comput. Chem. 1983, 4, 294-301.

[8] Francl, M. M.; Pietro, W. J.; Hehre, W. J.; Binkley, J. S.; Gordon, M. S.; DeFrees, D. J.; Pople, J. A. Self - consistent molecular orbital methods. XXIII. A polarization - type basis set for second - row elements. J. Chem. Phys. 1982, 77, 3654-3665.

[9] Fukui, K. The path of chemical reactions-the IRC approach. Acc. Chem. Res. 1981, 14, 363-368.

[10] Marenich, A. V.; Cramer, C. J.; Truhlar, D. G. Universal solvation model based on solute electron density and on a continuum model of the solvent defined by the bulk dielectric constant and atomic surface tensions. J. Phys. Chem. B 2009, 113, 6378-6396.

[11] (a) Krishnan, R.; Binkley, J. S.; Seeger, R.; Pople, J. A. Self - consistent molecular orbital methods. XX. A basis set for correlated wave functions. J. Chem. Phys. 1980, 72, 650-654. (b) McLean, A. D.; Chandler, G. S. Contracted Gaussian basis sets for molecular calculations. I. Second row atoms, $\mathrm{Z}=11-18$. J. Chem. Phys. 1980, 72, 5639-5648.

[12] CYLview, 1.0b; Legault, C. Y., Université de Sherbrooke, 2009 (http://www.cylview.org).

[13] Barman, M. K.; Das, K.; Maji, B. J. Org. Chem. 2019, 84, 1570-1579.

[14] Guo, Y.; Shen, Z. Org. Biomol. Chem., 2019, 17, 3103-3107.

[15] Hilf, S.; Grubbs, R. H.; Kilbinger, A. F. M. J. Am. Chem. Soc. 2008, 130, 11040-11048.

[16] Bouziane, A.; Hélou, M.; Carboni, B.;Carreaux, F.; Demerseman, B.; Bruneau, C.; Renaud, J.-L. Chem. Eur. J. 2008, 14, $5630-5637$.

[17] Liu, P.; Yasir, M.; Ruggi, A.; Kilbinger, A. F. M. Angew. Chem., Int. Ed. 2018, 57, 914-917.

[18] Yang, M.-H.; Orsi, D. L.; Altman, R. A. Angew. Chem. Int. Ed. 2015, 54, 2361-2365. 
[19] Balcells, S.; Haughey, M. B.; Walker, J. C. L.; Josa-Culleré, L.; Towers, C.; Donohoe, T. J. Org. Lett. 2018, 20, 3583-3586.

[20] Duboudin, J. G.; Jousseaume, B.; Saux, A. J. Organomet. Chem. 1979, $168,1$.

[21] Shoba, V. M.; Thacker, N. C.; Bochat, A. J.; Takacs, J. M. Angew. Chem., Int. Ed. 2016, 55, 1465-1469.

[22] Bober, A. E.; Proto, J. T.; Brummond, K. M. Org. Lett. 2017, 19, 1500-1503.

[23] Tien, C.-H.; Adams, M. R.; Ferguson, M. J.; Johnson, E. R. Org. Lett. 2017, 19, 5565-5568.

[24] Reed-Berendt, B. G.; Morrill, L. C. J. Org. Chem. 2019, 84, 3715-3724.

[25] Narczyk, A.; Pieczykolan, M.; Stecko, S. Org. Biomol. Chem. 2018, 16, 3921-3946.

[26] Ojo, O. S.; Miranda, O.; Baumgardner, K. C.; Bugarin, A. Org. Biomol. Chem., 2018, 16, 9354-9358.

[27] Nishina, N.; Yamamoto, Y. Angew. Chem., Int. Ed. 2006, 45, 3314-3317.

[28] Chakraborty, U.; Reyes-Rodriguez, E.; Demeshko, S.; Meyer, F.; Jacobi von Wangelin, A. Angew. Chem., Int. Ed. 2018, 57, 4970-4975.

[29] Takeuchi, R.; Ue, N.; Tanabe, K.; Yamashita, K.; Shiga, N. J. Am. Chem. Soc. 2001, 123, 9525-9534.

[30] Manßen, M.; Töben, I.; Kahrs, C.; Bölte, J.-H.; Schmidtmann,M.; Beckhaus, R. Organometallics 2017, 36, 2973-2981.

[31] Qi, X.; Zheng, T.; Zhou, J.; Dong, Y.; Zuo, X.; Li, X.; Sun, H.; Fuhr, O.; Fenske, D. Organometallics, 2019, 38, $268-277$.

[32] Jakhar, V. K.; Barman, M. K.; Nembenna, S. Org. Lett. 2016, 18, 4710-4713.

[33] Stach, T.; Dräger, J.; Huy, P. H. Org. Lett. 2018, 20, 2980-2983.

[34] Mao, S.; Chen, Z.; Wang, L.; Khadka, D. B.; Xin, M.; Li, P.; Zhang, S. Q. J. Org. Chem. 2019, 84, 463-471.

[35] Tan, X.; Wang, G.; Zhu, Z.; Ren, C.; Zhou, J.; Lv, H.; Zhang, X.; Chung, L. W.; Zhang, L.; Zhang, X. Org. Lett. 2016, 18, $1518-1521$.

[36] Grünberg, M. F.; Gooßen, L. J. Chem.- Eur. J. 2013, 19, 7334-7337.

[37] Murai, N.; Yonaga, M.; Tanaka, K. Org. Lett. 2012, 14, 1278-1281.

[38] Bucci, E.; De Napoli, L.; Di Fabio, G.; Messere, A.; Montesarchio, D.; Romanelli, A.; Picciallia, G.; Varra, M. Tetrahedron 1999, $55,14435$.

[39] Szostak, M.; Spain, M.; Procter, D. J. Org. Lett. 2012, 14, 840-843.

[40] Weber, S.; Brüig, J.; Zeindlhofer, V.; Schröder, C.; Stöger, B.; Limbeck, A.; Kirchner, K.; Bica, K. ChemCatChem 2018, 10, $4386-4394$.

[41] Firmenich SA; Berthier, D.; Herrmann, A.; Paret, N.; Ouali, L. US9334464, 2016, B2

[42] Bergner, M.; Duquette, D. C.; Chio, L.; Stoltz, B. M. Org. Lett. 2015, 17, 3008-3010.

[43] Wu, J.; Talwar, D.; Johnston, S.; Yan, M.; Xiao, J. Angew. Chem., Int. Ed. 2013, 52, 6983-6987.

[44] Aboo, A. H.;Bennett, E. L.; Deeprose, M.; Robertson, C. M.; Iggo, J. A.; Xiao, J. Chem. Commun. 2018, 54, 11805-11808.

[45] Sajid, M.; Kehr, G.; Daniliuc, C. G.; Erker, G. Chem. - Eur. J. 2015, 21, 1454-1457.

[46] Amamoto, Y.; Aoi, Y.; Nagashima, N.; Suto, H.; Yoshidome, D.;Arimura, Y.; Osakabe, A.; Kato, D.; Kurumizaka, H.; Kawashima, S.A.;

Yamatsugu, K.; Kanai, M. J. Am. Chem. Soc. 2017, 139, 7568-7576.

[47] Gavel, M.; Courant, T.; Yvan, A.; Joosten, P.; Lecourt, T. Org. Lett. 2019, 21, 1948-1952.

[48] Battilocchio, C.; Hawkins, J. M.; Ley, S.V. Org. Lett. 2013, 15, 2278-2281.

[49] de Koning, P. D.; Jackson, M.; Lennon, C. Org. Process Res. Dev. 2006, 10, 1054-1058.

[50] Mix, K. A.; Raines, R. T. Org. Lett. 2015, 17, 2358-2361.

[51] Takale, B. S.; Tao, S.; Yu, X. Q.; Feng, X.; Jin, T.; Bao, M.; Yamamoto, Y. Org. Lett. 2014, 16, 2558-2561.

[52] Das, K.; Mondal, A.; Pal, D.; Srivastava, H. K.; Srimani, D. Organometallics, 2019, 38, 1815-1825.

[53] Fan, L.; Jia, J.; Hou, H.; Lefebvre, Q.; Rueping, M. Chem. -Eur. J. 2016, 22, 16437-16440.

[54] Vivancos, Á.; Beller, M.; Albrecht, M. ACS Catal. 2018, 8, 17-21.

[55] Kuwano, R.; Ikeda, R.; Hirasada, K. Chem. Commun. 2015, 51, 7558-7561.

[56] Sahoo, B.; Kreyenschulte, C.; Agostini, G.; Lund, H.; Bachmann, S.; Scalone, M.; Junge, K.; Beller, M. Chem. Sci. 2018, 9, 8134-8141.

[57] Sorribes, I.; Liu, L.; Doménech-Carbó, A.; Corma, A. ACS Catal. 2018, 8, 4545-4557.

[58] Vilhanová, B.; van Bokhoven, J. A.; Ranocchiari, M. Adv. Synth. Catal. 2017, 359, 677-686.

[59] Ye, T.-N.; Li, J.; Kitano, M.; Hosono, H. Green Chemistry 2017, 19, 749-756.

[60] Adam, R.; Cabrero-Antonino, J. R.; Spannenberg, A.; Junge, K.; Jackstell, R.; Beller, M. Angew. Chem. Int. Ed. 2017, 56, 3216.

[61] Taisho Pharmaceutical Co. Ltd. - EP2172453, 2010, A1. 
[62] Wang, Y.; Huang, Z.; Leng, X.; Zhu, H.; Liu, G.; Huang, Z. J. Am. Chem. Soc. 2018, 140, 4417-4429.

[63] Yang, C.-H.;Chen, X.; Li, H.; Wei, W.; Yang, Z.; Chang, J. Chem. Commun. 2018, 54, 8622-8625.

[64] Eisenberger, P.; Bestvater, B. P.; Keske, E. C.; Crudden, C. M. Angew. Chem. Int. Ed. 2015, 54, $2467-2471$.

[65] Chen, F.; Surkus, A.-E.; He, L.; Pohl, M.-M.; Radnik, J.; Topf, C.; Junge, K.; Beller, M. J. Am. Chem. Soc. 2015, 137, 11718-11724.

[66] Wu, J.; Barnard, J. H.; Zhang, Y.; Talwar, D.; Robertson, C. M.; Xiao, J. Chem. Commun. 2013, 49, $7052-7054$.

[67] Deraedt, C.; Ye, R.; Ralston, W. T.; F Toste,. D.; Somorjai, G. A. J. Am. Chem. Soc. 2017, 139, 18084-18092.

[68] Scott, D. J.; Fuchter, M. J.; Ashley, A. E. Angew. Chem. Int. Ed. 2014, 53, 10218-10222.

[69] Wang,Y.; Dong, B.; Wang, Z.; Cong, X.; Bi, X. Org. Lett.2019, 21, 3631-3634.

[70] Chen, S.-P.; Deng, P.; Yuan, C.-F.; Yuan, L.-J. CrystEngComm, 2013,15, 1414-1420.

[71] Talwara, D., Li, H.-Y.; Durham, E.; Xiao, J. Chem. Eur. J., 2015, 21, 5370-5379.

[72] Ohigashi, A.; Temmaru, K.; Hashimoto, N. Org. Process Res. Dev. 2006, 10, 159-162.

[73] Kiselev, E.; Agama, K.; Pommier, Y.; Cushman, M. J. Med. Chem. 2012, 55, 1682-1697. 